\title{
A MODEL-BASED APPROACH TO HYPERSPECTRAL CHANGE DETECTION
}

\author{
Dissertation \\ Presented in Partial Fulfillment of the Requirements for the Degree \\ Doctor of Philosophy in the Graduate School of The Ohio State \\ University \\ By
}

Joseph Meola, B.S.E.E., M.S.E.E.

Graduate Program in Department of Electrical and Computer Engineering

The Ohio State University

2011

Dissertation Committee:

Dr. Randolph L. Moses, Advisor

Dr. Michael T. Eismann

Dr. Lee C. Potter

Dr. Philip Schniter 
(C) Copyright by

Joseph Meola

2011 


\begin{abstract}
Within the hyperspectral community, change detection is a continued area of interest. Change detection refers to the problem of identifying interesting changes that occur to a spatial area over which imagery has been collected on multiple occasions. Interesting changes in imagery typically correspond to changes in material reflectance associated with pixels in the scene, due e.g. to objects entering or leaving the scene. Complicating the problem of change detection is the presence of shadow, illumination, and atmospheric differences, as well as misregistration and parallax error, which often produce the appearance of a material change. Current change detection methods all suffer from similar weaknesses related to these nuisance changes.

To address these change detection difficulties, this dissertation proposes a modelbased approach to change detection in hyperspectral imagery. Using a physical model describing the sensor-reaching radiance, the change detection problem is formulated as a statistical hypothesis test in this work. This model-based change detection method incorporates model terms to account for both direct and diffuse shadow fractions to help mitigate false alarms associated with shadow differences between scenes. The resulting generalized likelihood ratio test provides an indicator of change at each pixel. This change detection approach is the first to utilize a physical model, which allows for the use of additional information beyond the statistics of the data for improved detection performance over baseline methods.
\end{abstract}


Using knowledge of hyperspectral data collection and calibration procedures, the data model and alternating optimization technique are extended for application to uncalibrated and relatively calibrated data. The model-based change detection method is applied to synthetically generated data, tower data, and airborne data with differing levels of calibration applied. The model-based approach demonstrates improvement in detection performance over baseline change detection algorithms for the data sets considered. Additionally, the model-based method provides estimates of model parameters, which can potentially be used for further data exploitation purposes.

Hyperspectral noise estimation is a sub-problem associated with the model-based change detection method. Hyperspectral data collected using charge-coupled devices or other photon detectors have sensor noise that is directly dependent on the amplitude of the signal collected. Additionally, the statistics of the noise can vary both spatially and spectrally as a result of camera characteristics and the calibration process applied to the data. Supervised and unsupervised noise estimation techniques are presented for estimating the noise statistics using calibration data or directly from the imagery if calibration data is unavailable. 
To my family, for their constant love and support. 


\section{Acknowledgments}

A number of important people have made this work possible. I would like to thank my adviser, Dr. Randy Moses, for taking time from his duties as Assistant Dean of Research to provide feedback and guidance for my research. Additionally, I would like to thank Dr. Mike Eismann who essentially has served as a co-adviser. He has provided support and guidance not only in my academic and professional life, but in my personal life as well. I would like to thank Dr. Josh Ash who has unofficially served as another co-adviser. Without his time and efforts, this research would not have been nearly as successful. I would like to thank my remaining dissertation committee members, Dr. Lee Potter, Dr. Phil Schniter, and Dr. Victor Jin for their time serving on my committee. I thank my co-workers and supervisors who have allowed me to take leave from my work duties to pursue this research. Finally, I thank my friends, family, and God, whose love and support have made this possible. I would specifically like to thank my parents. I cannot express how blessed I am to

have them as parents. I strive to be as loving and caring as they are and I thank God for them every day. 


\section{Vita}

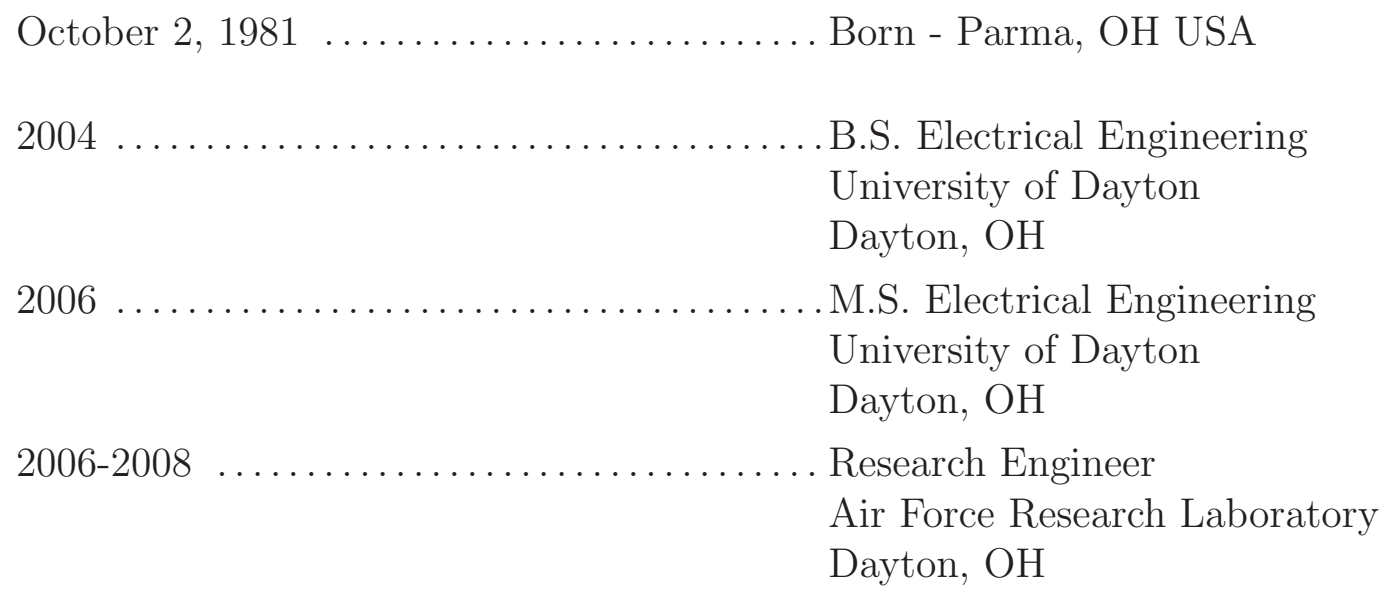

\section{Publications}

\section{Research Publications}

J. Meola, M. T. Eismann, R. L. Moses, J. N. Ash "A model-based approach to hyperspectral change detection". Proc. of SPIE, vol. 7695, no. 1., p. 76951G, 2010.

J. Meola, M. T. Eismann, R. L. Moses, J. N. Ash "Detecting changes in hyperspectral imagery using a model-based approach". IEEE Trans. on Geoscience and Remote Sensing, vol. 49, no. 7, pp.2647-2661, July 2011.

J. Meola, M. T. Eismann, R. L. Moses, J. N. Ash "Modeling and estimation of signal-dependent noise in hyperspectral imagery". Applied Optics, vol. 50, no. 21, pp. 3829-3846, 2011.

J. Meola, M. T. Eismann, R. L. Moses, J. N. Ash "Extension and implementation of a model-based approach to hyperspectral change detection". Proc. of SPIE, vol. 8048, no. 1., p. 804806, 2011. 
J. Meola, M. T. Eismann, R. L. Moses, J. N. Ash "Application of model-based change detection to airborne VNIR/SWIR hyperspectral imagery". Submitted for publication: IEEE Trans. on Geoscience and Remote Sensing, September 2011.

\section{Fields of Study}

Major Field: Electrical and Computer Engineering 


\section{Table of Contents}

Page

Abstract .................................. ii

Dedication . . . . . . . . . . . . . . . . . iv

Acknowledgments ........................ . . . v

Vita ..................................... vi

List of Tables . . . . . . . . . . . . . . . . . . . . . xi

List of Figures . . . . . . . . . . . . . . . . . . xii

1. Hyperspectral Change Detection Problem . . . . . . . . . . . . 1

1.1 Introduction . . . . . . . . . . . . . . . . . 1

1.2 Change Detection Applications . . . . . . . . . . . . . . . 2

1.3 Challenges in Hyperspectral Change Detection . . . . . . . . . 3

1.4 Current Methods for Hyperspectral Change Detection . . . . . . . 7

1.5 Contributions of the Dissertation . . . . . . . . . . . . 11

2. Development of Model-based Change Detection Approach . . . . . . . . 13

2.1 Introduction . . . . . . . . . . . . . . . . . . . 13

2.2 Data Model . . . . . . . . . . . . . . . . . . . 14

2.2.1 Full Data Model . . . . . . . . . . . . . . . . . . . . 14

2.2.2 Subspace Data Model . . . . . . . . . . . . . . . . 18

2.3 Change Detection Hypothesis Test . . . . . . . . . . . . . . . . . . 21

2.3.1 Hypothesis Test . . . . . . . . . . . . . . . 21

2.3.2 Generalized Likelihood Ratio Test . . . . . . . . . . . 25

2.4 Optimization Problem . . . . . . . . . . . . . . . . 28

2.4.1 Cost Function and Constraints . . . . . . . . . . . 29 
2.4 .2 Optimization Algorithm . . . . . . . . . . . . . . . 30

2.4 .3 Initialization . . . . . . . . . . . . . . . . 37

2.4 .4 Convergence Criteria . . . . . . . . . . . . . . . . . . . 39

2.4 .5 Performance Metrics . . . . . . . . . . . . . . . . . . 41

2.5 Spectral Smoothing of Reflectance . . . . . . . . . . . . 44

2.5.1 Smoothing via Filtering . . . . . . . . . . . . . . . 45

2.5.2 Smoothing via Subspace Constraint . . . . . . . . . . . 46

2.6 Extension to Uncalibrated and Relatively Calibrated Data . . . . 49

3. Hyperspectral Noise Estimation . . . . . . . . . . . . . . . . . . . 61

3.1 Introduction . . . . . . . . . . . . . . . . . 61

3.2 Hyperspectral Data and Calibration . . . . . . . . . . . 67

3.3 Theoretical Noise Model . . . . . . . . . . . . . . . . . . . . . . . . 68

3.3.1 Uncalibrated Data Noise Model . . . . . . . . . . . . . . 68

3.3.2 Calibrated Data Noise Model . . . . . . . . . . . . . . . . . 69

3.3.3 Spectral Correlation Characteristics . . . . . . . . . . 71

3.3.4 Signal to Noise Ratio . . . . . . . . . . . . . . . . . . . 73

3.4 Empirical Estimation of Noise Statistics . . . . . . . . . . . . . 74

3.4.1 Estimation from Calibration Data . . . . . . . . . 75

3.4 .2 Estimation from Imagery . . . . . . . . . . . . . . . 81

3.5 Significance of Noise Estimate for Change Detection . . . . . . . 99

4. Results . . . . . . . . . . . . . . . . . . . . . . . . . . . . 101

4.1 Introduction . . . . . . . . . . . . . . . . . . . . 101

4.2 MODTRAN Data . . . . . . . . . . . . . . . . . . . . 101

4.2 .1 Data Summary . . . . . . . . . . . . . . . . . . 102

4.2 .2 Noise Estimation . . . . . . . . . . . . . . . . . . 106

4.2 .3 Results . . . . . . . . . . . . . . . . . . . 107

4.3 AFRL Tower Data . . . . . . . . . . . . . . . . . . . . . 135

4.3 .1 Data Summary . . . . . . . . . . . . . . . . . 136

4.3.2 Results using Absolutely Calibrated Data Model . . . . . . 139

4.3.3 Results using Relatively Calibrated Data Model . . . . . . . 170

4.3.4 Results using Uncalibrated Data Model . . . . . . . . . . 172

4.4 HYDICE Data . . . . . . . . . . . . . . . . . . . . . . . . 186

4.4 .1 Data Summary . . . . . . . . . . . . . . . . . . . . 188

4.4 .2 Noise Estimation . . . . . . . . . . . . . . . . . . . . . 192

4.4.3 Results using Absolutely Calibrated Data Model . . . . . . 194

4.4.4 Results using Relatively Calibrated Data Model . . . . . . . 218

4.5 ARCHER Data . . . . . . . . . . . . . . . . . . . . . . 224

4.5.1 Data Summary . . . . . . . . . . . . . . . . . . 224 
4.5.2 Results using Relatively Calibrated Data Model . . . . . . . 227

4.5.3 Detection Results . . . . . . . . . . . . . . 237

4.6 Discussion . . . . . . . . . . . . . . . . . . . . . . . . . . . . . . . 241

5. Conclusion and Future Work . . . . . . . . . . . . . . . . . . . 245

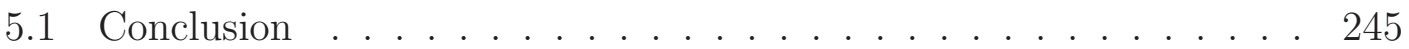

5.2 Future Work . . . . . . . . . . . . . . . . . 247

$\begin{array}{ll}\text { Appendices } & 249\end{array}$

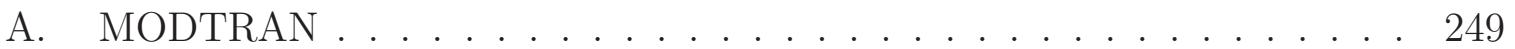

A.1 MODTRAN Background . . . . . . . . . . . . . . . . . 249

A.2 MODTRAN Operation . . . . . . . . . . . . . . . 250

A.2.1 Inputs . . . . . . . . . . . . . . . . . 250

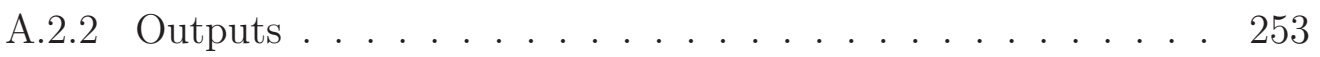

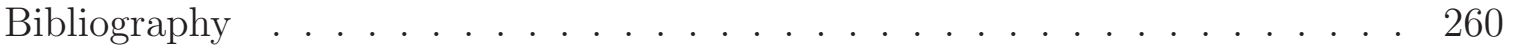




\section{List of Tables}

Table

Page

4.1 SCR for various algorithms using MODTRAN data set . . . . . . . . 134

4.2 Air Force Research Laboratory in-house sensor information . . . . . . 136

4.3 AFRL in-house data collection information . . . . . . . . . . 138

4.4 Signal to clutter ratio for various algorithms using AFRL data set . . 164

4.5 HYDICE Sensor Information . . . . . . . . . . . . . . . . . . . 188

4.6 Signal to clutter ratio for various algorithms using HYDICE data set 218

4.7 ARCHER sensor information . . . . . . . . . . . . . 225

4.8 ARCHER MAST data collection information . . . . . . . . . . . . 227

4.9 Signal to clutter ratio for various algorithms using ARCHER MAST data set . . . . . . . . . . . . . . . . . . . 240 


\section{List of Figures}

Figure $\quad$ Page

1.1 Variation in measured signal from vegetation material resulting from different physical processes . . . . . . . . . . . . . . .

1.2 Example of image change pair with illumination, shadowing, registra-

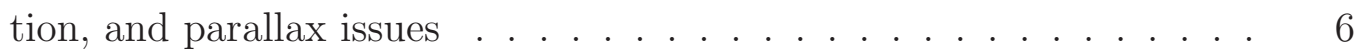

2.1 Simplified diagram of radiative transfer process for signal reaching sensor 15

2.2 Radiative transfer process with shadow terms introduced $\ldots \ldots . .17$

2.3 Flow chart for alternating optimization applied for model-based change detection . . . . . . . . . . . . . . . . . . . 32

2.4 Reflectance spectral for a number of materials, which exhibit spectrally smooth characteristics . . . . . . . . . . . . . . 45

2.5 Legendre Polynomials . . . . . . . . . . . . . . . . . . . . . . . . 48

2.6 Examination of subspace approximation for vegetation and camouflage reflectance . . . . . . . . . . . . . . . . . . . 48 48

2.7 General operation of pushbroom imaging spectrometer. . . . . . . 50

2.8 Flow chart for alternating optimization applied for model-based change detection for extended data model . . . . . . . . . . . . . . . 56

2.9 Example of geocorrection applied to airborne imagery to correct rolling of aircraft during collection . . . . . . . . . . . . . . 59

3.1 Linear fit to empirical data for noise variance at $m=170, \lambda_{k}=0.6 \mu m \quad 76$ 
3.2 Estimated noise model parameters for uncalibrated noise variance given in $(3.5) \ldots \ldots \ldots \ldots$. . . . . . . . . . . . . . . . . . . . . . .

3.3 Estimated noise model parameters for uncalibrated noise variance given in (3.5) using only 2 light levels . . . . . . . . . . . . . . . . . . 78

3.4 Estimated noise model parameters for uncalibrated noise variance given in (3.5) using 2 light levels while utilizing spatial independence . . . . 79

3.5 Estimated NUC gain term $\hat{a}[m, k]$ used for calibrating AFRL data. . $\quad 79$

3.6 Camera response and integrating sphere output affecting NUC gain applied . . . . . . . . . . . . . . . . . 80

3.7 Estimated gain and offset for noise variance model of calibrated data 81

3.8 Comparison of fraction of total noise resulting from signal-independent and signal-dependent terms as a function of signal level . . . . . . . . 82

3.9 Color image of AFRL in-house hyperspectral data . . . . . . . . . 85

3.10 Scatter plot of sample variance versus sample mean . . . . . . . . . 87

3.11 Contour plot of theoretical joint-distribution of sample mean and sample variance . . . . . . . . . . . . . . . . . . . . . . . 87

3.12 Comparison of noise model gain and offset estimates obtained using calibration data and supervised scatter plot method . . . . . . . . .

3.13 Scatter plot of sample mean versus sample variance using neighbor difference data to estimate sample variance . . . . . . . . . . . . . . . 90

3.14 Scatter plot thresholding and fit . . . . . . . . . . . . . . . 91

3.15 Binning of pixels in scene . . . . . . . . . . . . . . . . . . 92

3.16 Comparison of noise model gain and offset estimates obtained using calibration data and unsupervised scatter plot method . . . . . . .

3.17 Sample mean versus sample variance estimated using all panel pixels 
3.18 Comparison of noise model gain and offset estimates obtained using calibration data and in-scene methods . . . . . . . . . . . . . 95

3.19 Comparison of noise model gain and offset estimates obtained using calibration data and modified Green method . . . . . . . . . . . . . . 97

3.20 Comparison of noise model gain and offset estimates obtained using calibration data and modified Roger method . . . . . . . . . . . . . . 98

4.1 Material reflectance truth data . . . . . . . . . . . . . . . 103

4.2 Shadow coefficient truth for time-1 MODTRAN data . . . . . . . . . 104

4.3 Shadow coefficient truth for time-2 MODTRAN data . . . . . . . . . 104

4.4 Target truth for MODTRAN data . . . . . . . . . . . . . . 105

4.5 Color images of simulated noisy MODTRAN data . . . . . . . . 106

4.6 Estimated noise model gain and offset obtained using unsupervised scatter plot method for MODTRAN data . . . . . . . . . . . . . . . 107

4.7 Initial estimates of RT terms for MODTRAN data compared with truth108

4.8 Convergence criteria as a function of iteration for AFRL data . . . . 110

4.9 Initial estimates for time-1 shadow coefficients using bottom $5 \%$ of spectral norm pixels for MODTRAN data . . . . . . . . . . . . . 111

4.10 Initial estimates for time-2 shadow coefficients using bottom $5 \%$ of spectral norm pixels for MODTRAN data . . . . . . . . . . . . . . . 111

4.11 RT estimates for time-1 MODTRAN data for different reflectance estimation modalities using $5 \%$ shadow initialization. . . . . . . . . . . 112

4.12 RT estimates for time-2 MODTRAN data for different reflectance estimation modalities using $5 \%$ shadow initialization. . . . . . . . 113

4.13 Comparison of predicted measurement $\hat{L}$ with true measurement for dry grass pixel using 5\% shadow initialization for MODTRAN data . 114 
4.14 Comparison of predicted measurement $\hat{L}$ with true measurement for oak leaf pixel using $5 \%$ shadow initialization for MODTRAN data . . 115

4.15 Comparison of estimated reflectance $\rho$ with true reflectance for grass pixel and oak leaf pixel using $5 \%$ shadow initialization for MODTRAN data . . . . . . . . . . . . . . . . . . . 115

4.16 Estimated shadow coefficients for time-1 using $5 \%$ shadow initialization for MODTRAN data . . . . . . . . . . . . . . . . . 116

4.17 Estimated shadow coefficients for time- 2 using $5 \%$ shadow initialization for MODTRAN data . . . . . . . . . . . . . . . . . 117

4.18 Estimated total downwelling radiance for MODTRAN data . . . . . 118

4.19 Underestimated reflectance for dry grass pixel due to poor shadow initialization for MODTRAN data . . . . . . . . . . . . 118

4.20 Sample distribution of detection statistic for different reflectance estimation modalities . . . . . . . . . . . . . . . . . . 119

4.21 Empirical ROC curve performance for MODTRAN data using 5\% shadow initialization . . . . . . . . . . . . . . 120

4.22 Comparison of detection statistic images for $\mathrm{CC}$ and $\mathrm{CE}$ change detection algorithms for MODDTRAN data . . . . . . . . . . . . 121

4.23 Comparison of detection statistic images for different MB reflectance modalities using $5 \%$ shadow initialization for MODTRAN data . . . . 122

4.24 Comparison of reflectance spectrum for terra cota material and red brick material used in MODTRAN data . . . . . . . . . . . . 123

4.25 Initial estimates for time- 1 shadow coefficients using bottom $10 \%$ of spectral norm pixels for MODTRAN data . . . . . . . . . . . 124

4.26 Initial estimates for time-2 shadow coefficients using bottom $10 \%$ of spectral norm pixels for MODTRAN data . . . . . . . . . 125 
4.27 RT estimates for time-1 MODTRAN data for different reflectance estimation modalities using $10 \%$ shadow initialization. . . . . . . . . 126

4.28 RT estimates for time-2 MODTRAN data for different reflectance estimation modalities using $10 \%$ shadow initialization. . . . . . . . . 127

4.29 Estimated shadow coefficients for time-1 data using $10 \%$ shadow initialization for MODTRAN data . . . . . . . . . . . . . . . 128

4.30 Estimated shadow coefficients for time- 2 data using $10 \%$ shadow initialization for MODTRAN data . . . . . . . . . . . . . . . . . 128

4.31 Empirical ROC curve performance for MODTRAN data using 10\% shadow initialization . . . . . . . . . . . . . . . . . . . . . . . . 129

4.32 Comparison of detection statistic images for different MB reflectance modalities using $10 \%$ shadow initialization for MODTRAN data . . 130

4.33 Empirical ROC curve performance for MODTRAN data using true initialization . . . . . . . . . . . . . . . . . 130

4.34 Comparison of SCR for different MB reflectance modalities using 5\% shadow initialization for MODTRAN data . . . . . . . . . . . . . 131

4.35 Comparison of SCR for different MB reflectance modalities using $10 \%$ shadow initialization for MODTRAN data . . . . . . . . . . . 132

4.36 Comparison of SCR for different MB reflectance modalities using true initialization for MODTRAN data . . . . . . . . . . . . . . . . 132

4.37 Comparison of SCR for $\mathrm{CC}$ and $\mathrm{CE}$ for MODTRAN data . . . . . . 133

4.38 SCR as a function of subspace dimensionality $I$ for MODTRAN data. 135

4.39 Change pair collected with AFRL in-house, tower-based VNIR hyperspectral sensor . . . . . . . . . . . . . . . . . . 137

4.40 Lab reflectance spectra for green tarp, camouflage tarp, and grass for AFRL data . . . . . . . . . . . . . . . . . . 138 
4.41 Comparison of measured signal associated with change pixel locations at time-1 and time-2 for AFRL data . . . . . . . . . . . . . 139

4.42 Initial estimates of radiance for grass pixel as compared with true measurements for AFRL data . . . . . . . . . . . . . . . . . . . . 141

4.43 Convergence criteria as a function of iteration for AFRL data . . . . 142

4.44 Initial estimates for time-1 shadow coefficients using bottom $5 \%$ of spectral norm pixels for AFRL data . . . . . . . . . . . . . . . 143

4.45 Initial estimates for time- 2 shadow coefficients using bottom $5 \%$ of spectral norm pixels for AFRL data . . . . . . . . . . . . . . 144

4.46 RT estimates for time-1 AFRL data for different reflectance estimation modalities using $5 \%$ shadow initialization. . . . . . . . . . . 145

4.47 RT estimates for time-2 AFRL data for different reflectance estimation modalities using $5 \%$ shadow initialization . . . . . . . . . . 146

4.48 Estimated shadow coefficients for time-1 data using $5 \%$ shadow initialization for AFRL data . . . . . . . . . . . . . . . . . 147

4.49 Estimated shadow coefficients for time-2 data using $5 \%$ shadow initialization for AFRL data . . . . . . . . . . . . . . . . . . . . 147

4.50 Comparison of estimated measurement $\hat{L}$ with true measurement $L$ for grass pixel using $5 \%$ shadow initialization for AFRL data . . . . . 148

4.51 Comparison of estimated measurement $L$ with true measurement for green panel pixel using 5\% shadow initialization for AFRL data . . . 149

4.52 Comparison of estimated reflectance using $5 \%$ shadow initialization for AFRL data . . . . . . . . . . . . . . . . . . 150

4.53 Empirical ROC curve performance for AFRL data using $5 \%$ shadow initialization . . . . . . . . . . . . . . . . . . . 151

4.54 Comparison of detection statistic images for $\mathrm{CC}$ and $\mathrm{CE}$ change detection algorithms for AFRL data . . . . . . . . . . . . 152 
4.55 Comparison of detection statistic images for different MB reflectance modalities using $5 \%$ shadow initialization for AFRL data . . . . . . .

4.56 Initial estimates for time-1 shadow coefficients using bottom $10 \%$ of spectral norm pixels for AFRL data . . . . . . . . . . . . . . . . 154

4.57 Initial estimates for time- 2 shadow coefficients using bottom $10 \%$ of spectral norm pixels for AFRL data . . . . . . . . . . . . . . . . . 154

4.58 RT estimates for time-1 AFRL data for different reflectance estimation modalities using $10 \%$ shadow initialization. . . . . . . . . . . .

4.59 RT estimates for time-2 AFRL data for different reflectance estimation modalities using $10 \%$ shadow initialization. . . . . . . . . . . . 156

4.60 Estimated shadow coefficients for time-1 data using 10\% shadow initialization for AFRL data . . . . . . . . . . . . . . . . 157

4.61 Estimated shadow coefficients for time-2 data using 10\% shadow initialization for AFRL data . . . . . . . . . . . . . . . . 158

4.62 Comparison of estimated measurement $\hat{L}$ with true measurement $L$ for grass pixel using $10 \%$ shadow initialization for AFRL data . . . . . . 158

4.63 Comparison of estimated measurement $\hat{L}$ with true measurement $L$ for green panel pixel using 10\% shadow initialization for AFRL data . .

4.64 Comparison of estimated reflectance using 10\% shadow initialization for AFRL data . . . . . . . . . . . . . . . . . . . . 160

4.65 Empirical ROC curve performance for AFRL data using 10\% shadow initialization . . . . . . . . . . . . . . . . . 160

4.66 Comparison of detection statistic images for different MB reflectance modalities using 10\% shadow initialization for AFRL data . . . . . . 161

4.67 Comparison of SCR for different MB reflectance modalities using 5\% shadow initialization for AFRL data . . . . . . . . . . . . . . . 162

4.68 Comparison of SCR for different MB reflectance modalities using $10 \%$ shadow initialization for AFRL data . . . . . . . . . . . . . . . 163 
4.69 Comparison of SCR for CC and CE algorithms for AFRL data . . . . 163

4.70 Comparison of detection performance using a signal-dependent noise model (NF) versus a white noise model (NF-W) for AFRL data . . . 165

4.71 Comparison of detection statistic images using a signal-dependent noise model versus a white noise model for AFRL data . . . . . . . . . . . 166

4.72 RT estimates for time-1 AFRL data using a signal-dependent noise model $(\mathrm{NF})$ versus a white noise model $(\mathrm{NF}-\mathrm{W})$. . . . . . . . . . . . 167

4.73 RT estimates for time-2 AFRL data using a signal-dependent noise model $(\mathrm{NF})$ versus a white noise model $(\mathrm{NF}-\mathrm{W})$. . . . . . . . . . . . 168

4.74 Estimated shadow coefficients for time-1 data using white noise model for AFRL data . . . . . . . . . . . . . . . . . . . . . . . . 169

4.75 Estimated shadow coefficients for time-2 data using white noise model for AFRL data . . . . . . . . . . . . . . . . . . 169

4.76 Estimated relative gain $\mathbf{a}_{g}^{(t)}$ using relatively calibrated data model on calibrated AFRL data . . . . . . . . . . . . . . . . . 170

4.77 Estimated relative offset $\mathbf{b}_{g}^{(t)}$ using relatively calibrated data model on calibrated AFRL data . . . . . . . . . . . . . . 171

4.78 Estimated shadow coefficients for time-1 data using 5\% shadow initialization with relatively calibrated data model for AFRL data . . . . . 172

4.79 Estimated shadow coefficients for time-2 data using 5\% shadow initialization with relatively calibrated data model for AFRL data . . . . . 173

4.80 Estimate of time-1 effective sensor gain coefficient $\mathbf{a}^{(1)}$ in comparison with calibration data for AFRL data . . . . . . . . . . . . . . . 175

4.81 Estimate of time-2 effective sensor gain coefficient $\mathbf{a}^{(2)}$ in comparison with calibration data for AFRL data . . . . . . . . . . . . . . . 175

4.82 Estimate of time-1 dark current offset $\mathbf{b}^{(1)}$ in comparison with calibration data for AFRL data . . . . . . . . . . . . . . . . . . . 176 
4.83 Estimate of time-2 dark current offset $\mathbf{b}^{(2)}$ in comparison with calibration data for AFRL data . . . . . . . . . . . . . . . . . . . 177

4.84 Estimated shadow coefficients for time-1 data using 5\% shadow initialization with uncalibrated data model for AFRL data . . . . . . . . . 177

4.85 Estimated shadow coefficients for time-2 data using 5\% shadow initialization with uncalibrated data model for AFRL data . . . . . . . . . 178

4.86 Comparison of estimated reflectance for differing levels of calibration for AFRL data . . . . . . . . . . . . . . . . . . . . . . . . . . . . . 179

4.87 Empirical ROC curve performance for differing levels of calibration for AFRL data . . . . . . . . . . . . . . . . . . . . . 179

4.88 Comparison of detection statistic images for MB methods using the original data model, the relatively calibrated data model, and the uncalibrated data model for AFRL data . . . . . . . . . . . . . . . . 180

4.89 Estimated detector gain $\mathbf{a}^{(t)}$ obtained using NDVI in comparison with mean detector gain obtained from calibration data for AFRL data . . 181

4.90 Estimated dark current offset $\mathbf{b}^{(t)}$ obtained using NDVI in comparison with mean offset obtained from calibration data for AFRL data . . . 182

4.91 Estimated detector gain $\mathbf{a}^{(t)}$ obtained using NDVI for initialization for AFRL data . . . . . . . . . . . . . . . . . . . . 183

4.92 Estimated dark current offset $\mathbf{b}^{(t)}$ obtained using NDVI for initialization for AFRL data . . . . . . . . . . . . . . . . . . 183

4.93 Estimated shadow coefficients for time-1 data using 5\% shadow initialization with uncalibrated data model and NDVI initialization for AFRL data . . . . . . . . . . . . . . . . . . . . . . 184

4.94 Estimated shadow coefficients for time-2 data using 5\% shadow initialization with uncalibrated data model and NDVI initialization for AFRL data . . . . . . . . . . . . . . . . . . . . . 184 
4.95 Comparison of estimated reflectance for differing levels of calibration/initialization for AFRL data . . . . . . . . . . . . . . . . 185

4.96 Empirical ROC curve performance for differing levels of calibration/initialization for AFRL data . . . . . . . . . . . . . . 186

4.97 Comparison of detection statistic images for differing levels of calibration/initialization . . . . . . . . . . . . . . . . . . . . . . 187

4.98 Spectral resolution of HYDICE system . . . . . . . . . . . . . . . . . 189

4.99 HYDICE unregistered change pair . . . . . . . . . . . . . . . . . . . . 191

4.100HYDICE registered change pair . . . . . . . . . . . . . . . . . . 192

4.101Sample mean and variance of gray-level panels for a particular band in visual regime of HYDICE data . . . . . . . . . . . . . . . . . . . . . . 193

4.102Estimated gain and offset for noise model using automated scatter plot fitting method for HYDICE data . . . . . . . . . . . . . . . . . . . 194

4.103Estimated gain and offset for noise model using fit to sample mean and sample variance of gray panels for HYDICE data . . . . . . . . . . . 195

4.104Estimated gain and offset for noise model using fit to sample mean and sample variance of gray panels . . . . . . . . . . . . . . . . . . 195

4.105Material reflectance truth and initial radiance estimates for HYDICE data . . . . . . . . . . . . . . . 196

4.106Convergence criteria as a function of iteration for HYDICE data . . . 197

4.107Initial estimates for time-1 shadow coefficients using bottom $5 \%$ of spectral norm pixels for HYDICE data . . . . . . . . . . . . . . . 198

4.108Initial estimates for time-2 shadow coefficients using bottom $5 \%$ of spectral norm pixels for HYDICE data . . . . . . . . . . . . . . . . . 199

4.109RT estimates for time-1 HYDICE data for different reflectance estimation modalities using $5 \%$ shadow initialization . . . . . . . . . . 200 
4.110RT estimates for time-2 HYDICE data for different reflectance estimation modalities using $5 \%$ shadow initialization. . . . . . . . . . . 201

4.111Estimated shadow coefficients for time-1 data using $5 \%$ shadow initialization for HYDICE data . . . . . . . . . . . . . . . . . . 202

4.112Estimated shadow coefficients for time-2 data using $5 \%$ shadow initialization for HYDICE data . . . . . . . . . . . . . . . . . . . . 203

4.113Comparison of estimated measurement $\hat{L}$ with true measurement $L$ for grass pixel using $5 \%$ shadow initialization for HYDICE data . . . . 203

4.114Comparison of estimated measurement $L$ with true measurement for $64 \%$ gray panel pixel using $5 \%$ shadow initialization for HYDICE data 204

4.115Comparison of estimated reflectance using $5 \%$ shadow initialization for HYDICE data . . . . . . . . . . . . . . . . . . . . . . 204

4.116Empirical ROC curve performance using $5 \%$ shadow initialization for HYDICE data . . . . . . . . . . . . . . . . . . . 205

4.117Comparison of detection statistic images for $\mathrm{CC}$ and $\mathrm{CE}$ change detection algorithms for HYDICE data . . . . . . . . . . . 206

4.118Comparison of detection statistic images for different MB reflectance modalities using $5 \%$ shadow initialization for HYDICE data . . . . 207

4.119Initial estimates for time-1 shadow coefficients using bottom $10 \%$ of spectral norm pixels for HYDICE data . . . . . . . . . . . 208

4.120Initial estimates for time-2 shadow coefficients using bottom $10 \%$ of spectral norm pixels for AFRL data . . . . . . . . . . . . . . . 209

4.121RT estimates for time-1 HYDICE data for different reflectance estimation modalities using $10 \%$ shadow initialization. . . . . . . . . 210

4.122RT estimates for time-2 HYDICE data for different reflectance estimation modalities using $10 \%$ shadow initialization. . . . . . . . . . 211

4.123Estimated shadow coefficients for time-1 data using $10 \%$ shadow initialization for HYDICE data . . . . . . . . . . . . . . . . . . . 212 
4.124Estimated shadow coefficients for time-2 data using $10 \%$ shadow initialization for HYDICE data . . . . . . . . . . . . . . . . . . . . . . 212

4.125Comparison of estimated measurement $\hat{L}$ with true measurement $L$ for grass pixel using 10\% shadow initialization for HYDICE data . . . . . 213

4.126Comparison of estimated measurement $L$ with true measurement for $64 \%$ gray panel pixel using 10\% shadow initialization for HYDICE data 213

4.127Comparison of estimated reflectance using $10 \%$ shadow initialization for HYDICE data . . . . . . . . . . . . . . . . . . . . . . . . . 214

4.128Empirical ROC curve performance for HYDICE data using $10 \%$ shadow initialization . . . . . . . . . . . . . . . . 214

4.129Comparison of detection statistic images for different MB reflectance modalities using 10\% shadow initialization for HYDICE data . . . . . 215

4.130Comparison of SCR for different MB reflectance modalities using $5 \%$ shadow initialization for HYDICE data . . . . . . . . . . . . 216

4.131Comparison of SCR for different MB reflectance modalities using $10 \%$ shadow initialization for HYDICE data . . . . . . . . . . . . . . . 217

4.132Comparison of SCR for CC and CE algorithms for HYDICE data . . 217

4.133Estimated relative gain $\mathbf{a}_{g}^{(t)}$ for HYDICE data . . . . . . . . . . . 219

4.134Estimated relative offset $\mathbf{b}_{g}^{(t)}$ for HYDICE data . . . . . . . . . . 219

4.135RT estimates for time-1 HYDICE data for both original data model and relative calibration model . . . . . . . . . . . . . . 220

4.136RT estimates for time-2 HYDICE data for both original data model and relative calibration model . . . . . . . . . . . . . . . . . 221

4.137Estimated shadow coefficients for time-1 data using 5\% shadow initialization with relatively calibrated data model for HYDICE data . . . . 222 
4.138Estimated shadow coefficients for time-2 data using $5 \%$ shadow initialization with relatively calibrated data model for HYDICE data . . . . 223

4.139Empirical ROC curve performance using different data models for HYDICE data . . . . . . . . . . . . . . . . . . . . . . 223

4.140Comparison of detection statistic images for MB methods using original data model and relative calibration data model for HYDICE data . . 224

4.141ARCHER MAST change pair . . . . . . . . . . . . . 226

4.142Initial estimates for time-1 shadow coefficients using bottom $5 \%$ of spectral norm pixels for MAST data . . . . . . . . . . . . 228

4.143Initial estimates for time-2 shadow coefficients using bottom $10 \%$ of spectral norm pixels for MAST data . . . . . . . . . . . . . . 229

4.144General leaf reflectance spectrum used to obtain initial calibration term estimates using NDVI for MAST data . . . . . . . . . . . . . . 230

4.145Initial estimates of relative calibration gain and offset for ARCHER MAST data . . . . . . . . . . . . . . . . . . . . 230

4.146Estimated shadow coefficients for time-1 data using spatially constant calibration terms for MAST data . . . . . . . . . . . . . . . 231

4.147Estimated shadow coefficients for time-2 data using spatially constant calibration terms for MAST data . . . . . . . . . . . . . . . 232

4.148Estimated shadow coefficients for time-1 data using spatially adaptive calibration terms for MAST data . . . . . . . . . . . . . . 232

4.149Estimated shadow coefficients for time-2 data using spatially adaptive calibration terms for MAST data . . . . . . . . . . . . . . . 233

4.150RT estimates for time-1 MAST data for both spatially constant (NF) and spatially adaptive (NF-A) calibration terms . . . . . . . . . 234

4.151RT estimates for time-2 MAST data for both spatially constant (NF) and spatially adaptive (NF-A) calibration terms . . . . . . . 235 
4.152Final estimates of relative calibration gain and offset for ARCHER MAST data . . . . . . . . . . . . . . . . . . . . . . . 236

4.153Estimation results for a grass pixel for MAST data using spatially constant calibration terms and spatially adaptive calibration terms . . 237

4.154Comparison of detection statistic images for $\mathrm{CC}$ and $\mathrm{CE}$ change detection algorithms for MAST data . . . . . . . . . . . . . 238

4.155Comparison of detection statistic images for MB method on MAST data using relative calibration data model . . . . . . . . . . . . 238

4.156Empirical ROC curve performance for MB methods using relatively calibrated data model in comparison with $\mathrm{CC}$ and $\mathrm{CE}$ algorithms for MAST data . . . . . . . . . . . . . . . . . . . . . 239

A.1 Radiative transfer diagrams for direct and diffuse radiance incorporating nonlinear scattering and adjacency . . . . . . . . . 256

A.2 Radiative transfer diagrams for upwelled radiance incorporating nonlinear scattering and adjacency $\ldots \ldots \ldots \ldots . \ldots 256$ 


\section{Chapter 1: HYPERSPECTRAL CHANGE DETECTION PROBLEM}

\section{$1.1 \quad$ Introduction}

Musical artist William Fitzsimmons has a song entitled, "Everything has Changed" that contains the line "everything's different but nothing's changed," [31]. This brief song lyric summarizes the problem associated with digital image change detection. Simply stated, change detection involves comparison of digital images taken of the same spatial region collected on different occasions in order to determine what has "changed" over time.

From a philosophical standpoint, change can signify a number of ideas. For instance, if an individual glances outside of his office window at a field with some trees at mid-day and again before he leaves towards the end of the day, the individual might argue that the scene has not changed much over that span of time. Although several aspects may be different, such as the shadow positions, the physical composition of the scene essentially remains unchanged. However, if a digital camera makes the same observations at mid-day and late afternoon, an automated image comparison method might produce the result that indeed the scene has changed because the digital numbers produced for certain pixels at mid-day would not be the same as 
those produced later in the day due to shadows moving or other physical processes. What the camera lacks is the ability to interpret the nature of the change in the scene as it simply deals with digital numbers representing reflected illumination from the materials in the scene. While the term significant change is subject to interpretation, observers are typically interested in changes corresponding to objects being inserted or removed from a scene or cases where there is a change in material composition

or apparent optical properties. For electro-optical imaging sensors, these types of physical changes correspond to changes in material properties, such as reflectance. This signifies the basic hypothesis testing problem associated with change detection. Given multiple measurements of signals produced by a spatial region from a scene, one must decide between the null hypothesis, which signifies no change in material reflectance, or the alternative, which signifies a material reflectance change. Hyperspectral change detection (HSCD) forms a subclass of digital image change detection. Hyperspectral imaging (HSI) sensors measure radiance at a large number (typically 100 or more) of contiguous, narrow spectral bands of light. Consequently, the images provide much more reflectance information about the objects in the scene than broadband or color imagery, which allows for greater discrimination of objects and improved change detection performance.

\subsection{Change Detection Applications}

As mentioned previously, change detection algorithms are typically designed to identify pixels corresponding to spatial regions for which material reflectance has changed. Alterations in spectral reflectance are important because these types of changes typically result from the removal or insertion of an object associated with 
the pixel/s being observed, or chemical changes to the object itself. For instance, the reflectance of vegetation varies depending upon its health. Consequently, one important application of digital image change detection is monitoring of forest or crop health $[23,21]$. Similarly, one can use change detection as a method for monitoring rates of change to the ecosystem and pollution levels for lake and ocean environments [33]. For machine vision problems, change detection is often used as a method of monitoring the status of man-made structures [58, 41, 87]. Assuming the data rate and processing rate are high enough, change detection plays a very important role in many different video surveillance applications as well [22]. Recently, tracking using hyperspectral images has produced interest $[82,12]$ for which change detection concepts are required. In the medical field, change detection concepts are often applied to MRI data for detecting abnormalities within the body or the progression of an illness over time [67]. Finally, change detection is often used in improving target detection in difficult environments by employing concepts to help reduce background clutter [71]. With a wide range of applications, one can easily understand the importance of improving change detection techniques. False identification or missed identification of change can result in significant consequences for several of the applications listed above.

\subsection{Challenges in Hyperspectral Change Detection}

Many of the applications listed above share similar challenges in regard to change detection. Here, focus remains on passive airborne remote sensing applications in the visible through shortwave infrared spectral region for which the sun is the primary source of illumination in the scene. Ideally, one would like to compare two images in 
an automated fashion to accentuate important changes while suppressing other noninteresting changes. These "nuisance" changes result from a number of factors. The time period between data collections can vary from fractions of a second for tracking problems to days, weeks, or even months for ecosystem or land-use monitoring. Depending on the time delay, illumination conditions can vary between collections as a result of solar position and atmospheric transmission changes. A change in solar position alters both the shadow positions within the scene as well as the overall level of illumination reaching the scene and ultimately the sensor. The former are considered "local" illumination variations because shadow differences can be significant in some portions of the scene while nonexistent elsewhere, whereas the latter are considered "global" illumination variations across the entire scene. Solar illumination must travel through the atmosphere both on the downward path (to the scene) and the upward path (to the sensor) along which illumination is scattered and absorbed. The degree to which the illumination level and spectral distribution vary depends upon the optical length of the path, which in turn depends on solar and sensor positions, and the transmission of the atmosphere. Atmospheric transmission can vary significantly over the course of a day, with very low transmission in the morning due to fog or haze from pollution to higher transmission at mid-day. As an example, Figure 1.1(a) displays the signal measured from vegetation at two different times. The solar position, visibility, and water content of the atmosphere have changed between times. As a result, the signal produced by the same material is significantly altered as a result of global illumination changes. Conversely, Figure 1.1(b) displays the signal measured from grass at two different locations in the same scene; the first location in shadow and the second location fully illuminated. Although the atmospheric and global illumination 


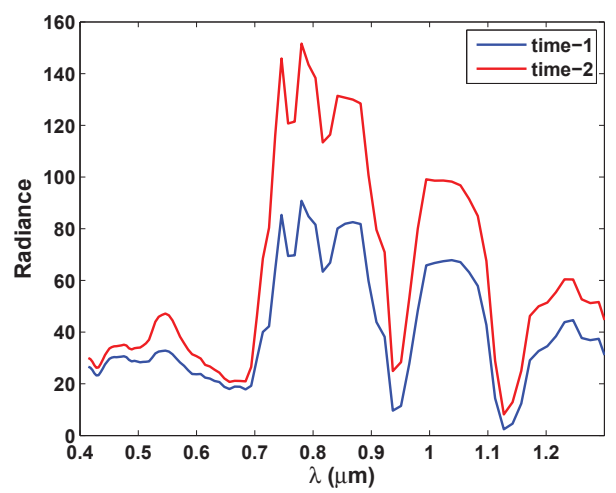

(a) Global illumination change

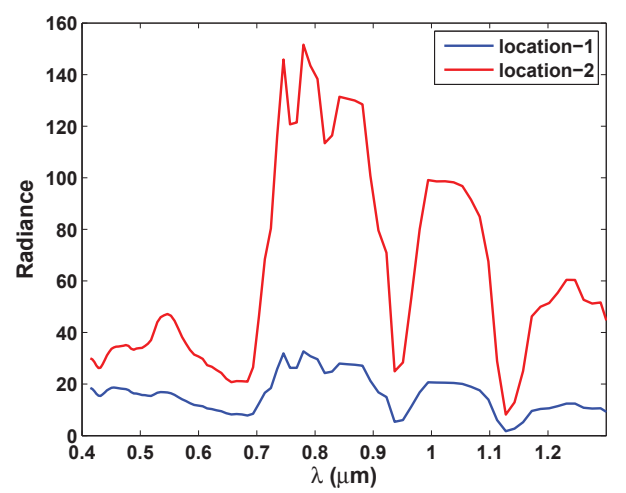

(b) Local illumination change

Figure 1.1: Variation in measured signal from vegetation material resulting from different physical processes

conditions are the same, the measured signal varies due to shadows.

Additionally, changes in sensor position produce further nuisance changes in the form of misregistration and parallax error. The problem considered in this work is pixel-level change detection which implies a pixel-by-pixel comparison between images to determine if change has occurred for each pixel. The reason for considering pixellevel detection is that, for many applications, the object or change of interest is small in physical size, and may occupy only a single pixel in the scene. The comparison assumes the images have been registered and each pixel pair provides a measurement from the same spatial region in the scene. For airborne systems, operators cannot possibly fly the exact same pattern on two separate occasions with the same height, pitch, yaw, and roll of the aircraft. Flight path variations cause misregistration between the images being compared. While advanced image registration methods exist that can reduce misregistration to subpixel levels [72], in many cases residual misregistration can introduce falsely identified changes. Similarly, changes in sensor viewing 
geometry can lead to parallax error, which is an apparent displacement of the position of an object when viewed along a different line of sight [76]. Consequently, objects within a scene can appear to move relative to each other if the sensor itself has moved. All of these factors contribute to nuisance changes increasing the complexity of the change detection problem. Figure 1.2 displays a simulated image pair demonstrating several of the difficulties associated with change detection. The pair has one interesting change (the insertion of a vehicle) along with the "nuisance" changes described above associated with varying solar position and a slight difference in sensor viewing geometry.

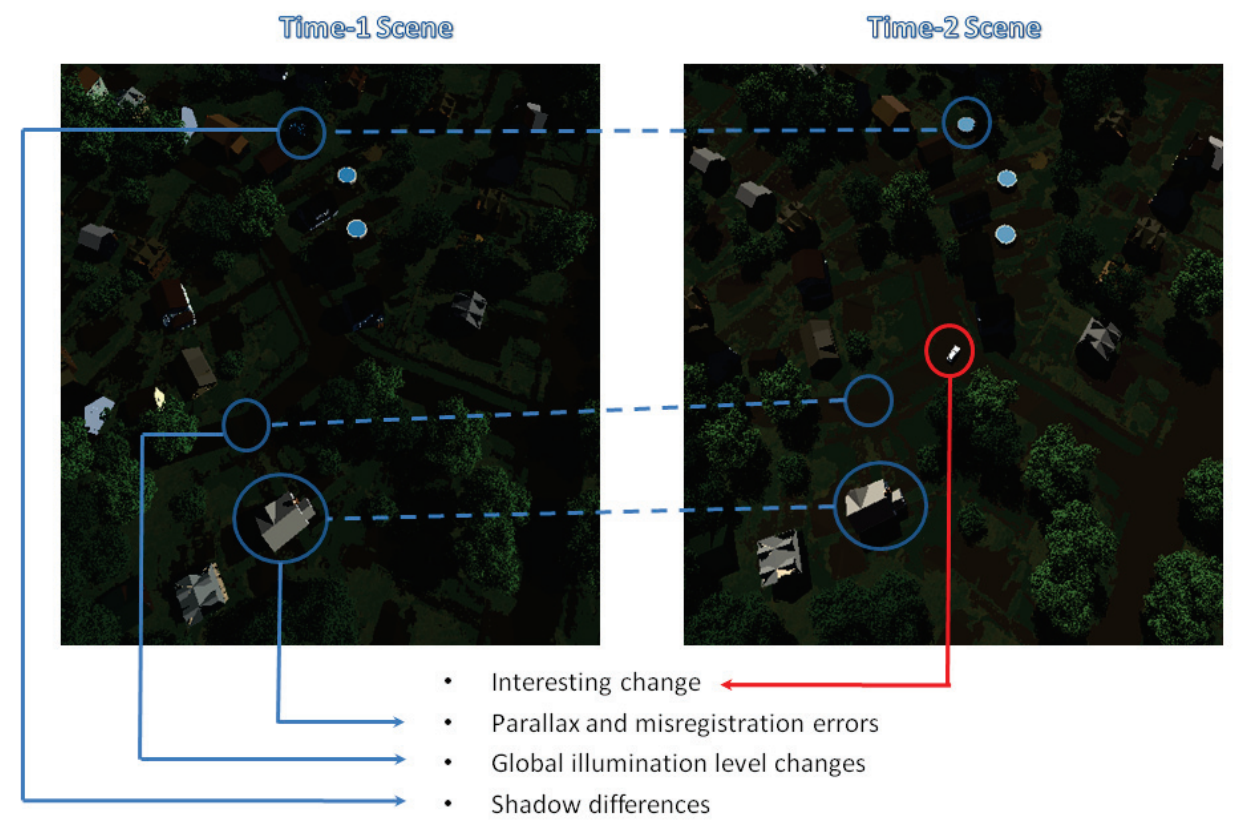

Figure 1.2: Example of image change pair with illumination, shadowing, registration, and parallax issues 


\subsection{Current Methods for Hyperspectral Change Detection}

As previously mentioned, the problem of digital image change detection has applications in many different fields ranging from surveillance and tracking to remote sensing and medical imaging. Consequently, the body of literature discussing change detection methods is quite large. Change detection is closely related to many problems in Computer Vision with additional literature available in this field. Radke [65] and Singh [79] both provide extensive reviews of various digital image change detection methods, classifying them into various groups. Singh's work focuses primarily on change detection methods as applied for remote sensing problems whereas Radke's more recent work covers a more general field of change detection problems. Several concepts reviewed by Radke applied to single band or gray-scale data have found their way for use in the hyperspectral problem as well. Key among these concepts is the idea of modeling the intensity measured for a pixel as the product of an illumination term (which varies between scenes) and a reflectance term (which does not vary) [64]. Additional work examines modeling an image collected at one time as a linear function of the image collected at an earlier time to account for varying illumination [59]. A complete review of change detection methods for all fields is beyond the scope

of this work. Instead, focus is given mainly on change detection methods that have been applied to multi-spectral and hyperspectral data problems.

Many currently employed change detection algorithms fall into the class of quadratic covariance-based change detection methods [86]. This class of detector outputs a non-negative scalar value $\mathcal{D}$ as a measure of the change between two hyperspectral 
measurement vectors, $\mathbf{L}^{(1)}$ and $\mathbf{L}^{(2)}$ given by the form,

$$
\mathcal{D}\left(\mathbf{L}^{(1)}, \mathbf{L}^{(2)}\right)=\left[\begin{array}{ll}
\left(\mathbf{L}^{(1)}\right)^{T} & \left(\mathbf{L}^{(2)}\right)^{T}
\end{array}\right] \mathbf{Q}\left[\begin{array}{l}
\mathbf{L}^{(1)} \\
\mathbf{L}^{(2)}
\end{array}\right]
$$

where different methods arise from different particular choices of the matrix $\mathbf{Q}$. The spectral measurement vectors correspond to the signal measured from a single spatial pixel at various wavelength bands of illumination. These methods are referred to as covariance-based as they typically use spectral correlations between the data to help identify change. Correspondingly, these approaches require knowledge of the covariance and cross-covariance matrices, which are typically estimated from the data. While the specific details of the structure of $\mathbf{Q}$ are omitted here, the basic formulation of several quadratic covariance-based detectors is discussed. For the simplest case of change detection when the operator has little reason to believe that significant illumination or environmental differences exist, the data sets are simply subtracted pixel by pixel and a distance metric is applied to the difference vectors. The most common distance metric applied is a spatially-adaptive Mahalanobis distance, referred to as the RX algorithm, which is derived from the assumption that within a small spatial window, any residual difference is due to zero-mean multi-variate Gaussian sensor noise $[66,42,51]$. This simple difference approach often results in significant false alarms due to the common nuisance changes discussed earlier.

A much more favorable and popular approach to change detection is to apply a gain matrix and offset vector to one or both of the spectral measurement vectors to account for the global illumination variations discussed earlier before differencing, which is similar to the concepts reviewed by Radke [65]. Often the gain matrix and offset vector are applied to one data set to try and "predict" how the measured signal would appear under the same atmosphere and illumination conditions of the second 
data set. Early work along these lines was geared towards using change detection to improve target detection in long-wave infrared (LWIR) multi-spectral images for cases in which the target signal is very similar to the background signal [73]. To improve target detection performance, a number of previous data sets that are assumed to have no target present are used to help remove the background signal from the data with the target present. Spectral correlation exists between the data sets because the assumption is made that the materials present for each pixel do not change over time. The method essentially utilizes the spectral correlations between the data sets to predict the natural evolution of the background statistics as a function of the illumination conditions. A linear minimum mean-squared error (MMSE) predictor (also called chronochrome (CC)) is applied to evolve the background statistics of the data with target present to match that of the data with no target. Afterwards, the background is subtracted thereby segmenting the target for detection.

In subsequent work, a more formal presentation is given to the evolution of spectral measurements using linear predictors [75]. A subsequent predictor known as covariance equalization (CE) is developed, which does not require cross-correlation statistics of the data sets, which potentially can be beneficial when residual misregistration exists. Analysis of the relative robustness of the predictors in the presence of misregistration does demonstrate an advantage in certain cases for use of CE [55]. The strength of these approaches is examined and extended for use in visible to nearinfrared (VNIR) hyperspectral data [74]. One of the main weaknesses associated with this approach is estimation of the prediction matrices, which requires second order statistics of the data. However, due to varying material classes and local illumination differences, these statistics are non-stationary across the scene. The author's Masters 
Thesis work $[54,56]$ demonstrates shortcomings of the prediction-based methods. To improve the performance of the prediction-based methods, clustering is utilized to segment the data into classes with similar statistics and separate predictors are estimated and applied for each spectral class [27, 29, 28]. Even with segmentation, these methods tend to suffer in the presence of local illumination differences, such as shadows. Other work applies more general nonlinear predictors to the problem such as neural networks [19], a generalized chronochrome derived from the joint distribution of the data [84], or simply the general nonlinear MMSE prediction based upon the conditional probability density function of the data [15].

Other quadratic covariance-based methods treat the two separate images as a single image with twice as many spectral bands and apply anomaly detection algorithms, such as the locally adaptive Mahalanobis distance described earlier, to identify measurements that do not fit the joint background model well. This approach does not differentiate between anomalous measurements in either image, such as a vehicle present at both times in a mainly vegetative environment, versus a measurement that is mutually anomalous (i.e. change). Consequently, further work applies an entropybased metric to accentuate the mutual information between the measurements [85]. Ultimately, the class of quadratic covariance-based approaches suffer from a few main weaknesses:

- The need to estimate non-stationary covariance matrices for the data.

- The inability to adequately handle local illumination differences such as shadows. 
- Natural spatial correlations present in the images are not exploited since each pixel is treated as an independent measurement.

Work exists in the use of Markov random fields (MRF) for utilizing spatial correlations in hyperspectral data for anomaly detection $[77,37]$ and for change detection in single band data by application on the difference image $[44,13]$. Recent work extends the MRF change detection application to color images and attempts to jointly incorporate spectral and spatial information with limited results available [18]. Overall, the literature does not contain much work in the application of MRF for hyperspectral data with any significant results. Additional work examines the use of canonical correlation analysis coupled with a spatial post-processing algorithm for use in hyperspectral change detection again with limited results [61,60].

\subsection{Contributions of the Dissertation}

As a result of the limitations of current methods, this work examines an approach to change detection driven by the physics of radiative transfer. The data model explicitly allows for differences in the measured signal resulting from global and local illumination changes. The latter are accounted for using scalar coefficients that parameterize the level of shadowing of each spatial pixel. Using this model, a composite hypothesis test is developed for change detection. This change detection approach is the first to utilize a physical model, which allows for the use of additional information beyond the statistics of the data for improved detection performance over the baseline methods discussed here. Since the physical model parameters are unknown, maximum likelihood estimates (MLE) of these parameters are obtained using the entire joint data set. The resulting generalized likelihood ratio test (GLRT) 
provides an indicator of change for each pixel in the joint data. In Chapter 2, the model-based change detection method is developed along with the subsequent GLRT and optimization problem. In Chapter 3, techniques are discussed for developing noise covariance estimates for use in the GLRT. In Chapter 4, the change detection method is applied to synthetic data generated using an atmospheric modeling tool and library material reflectance spectra, Air Force Research Laboratory (AFRL) tower-based data, Hyperspectral Digital Imagery Collection Experiment (HYDICE) airborne data and Airborne Real-time Cueing Hyperspectral Enhanced Reconnaissance (ARCHER) data. Finally, in Chapter 5, the results are summarized and future directions for this research are discussed. 


\section{Chapter 2: DEVELOPMENT OF MODEL-BASED CHANGE DETECTION APPROACH}

\section{$2.1 \quad$ Introduction}

In this chapter, a new method for HSCD is developed directly from a physical model describing the data. Shadow coefficients are included in the data model to account for the local illumination differences that tend to introduce false alarms. Using this model, a composite hypothesis test is applied for change detection. Since the physical model parameters are unknown, maximum likelihood estimates of these parameters are obtained using the entire joint data set. The resulting generalized likelihood ratio test provides an indicator of change for each pixel in the joint data. In Section 2.2, the data model is discussed along with a subspace simplification for the atmospheric and illumination components of the model. In Section 2.3, the hypothesis test for the change detection problem is developed along with the resulting GLRT. In Section 2.4, the optimization problem associated with the MLE is described along with a simplified method for handling the optimization. In Section 2.5, techniques

are discussed to produce spectrally smooth estimates of material reflectance, which 
can ultimately lead to improved estimation performance. In Section 2.6, the modelbased approach is extended for use on uncalibrated imagery and relatively calibrated imagery.

\subsection{Data Model}

In order to understand the change detection problem more completely, a physical model is developed that describes the signal reaching the remote sensor. The data model describes the relationship between the illumination collected at the sensor to the parameter of interest, the material spectral reflectance.

\subsubsection{Full Data Model}

Material reflectance can simply be defined as the ratio of illumination reflected by a surface to the illumination incident upon it. Generally speaking, the reflectance of a material depends upon the wavelength of illumination $\lambda$, the surface structure, the angle of illumination, the angle of observation, the polarization of illumination, and the temperature and pressure of the material. However, Lambertian scattering assumptions are often made to reduce the dependency to that of wavelength alone [76]. The spectral reflectance of a material can possess unique spectral features useful for identification purposes. As a result, a change in spectral reflectance can signify a change in material. The illumination, characterized by a spectral radiance $L$, reaching the sensor aperture is directly related to the reflectance of the materials within the scene. This sensor-reaching radiance, which is a combination of reflected radiance and other terms, is collected by the sensor and focused onto an array of detectors or pixels. Consequently, the measured signal produced by each spatial pixel is directly related to spectral radiance reflected by a specific spatial region in the scene. 
Other interference terms contribute to the measured signal as well. Figure 2.1 displays a simplified diagram of the radiative transfer process accounting for the signal reaching the sensor. The illumination incident upon the surface of the material with

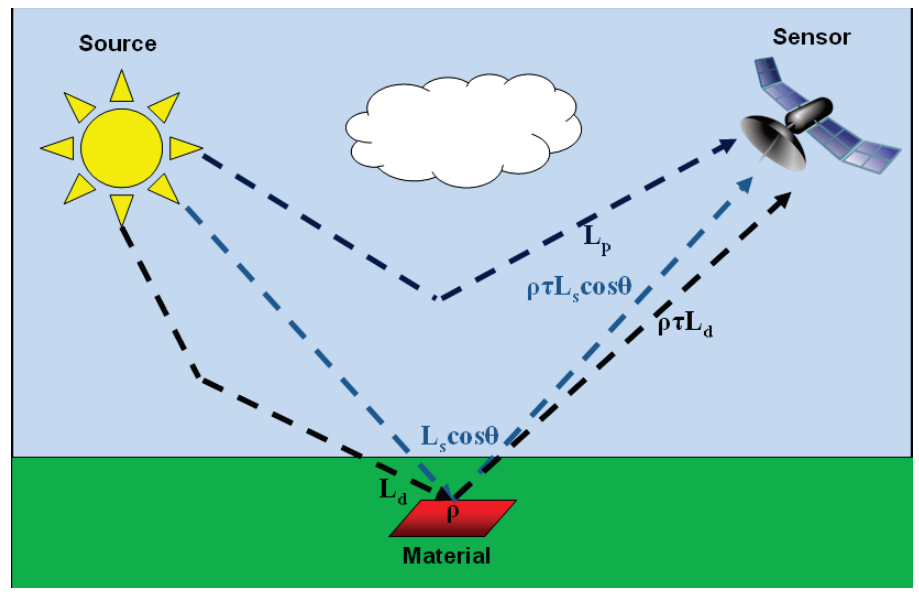

Figure 2.1: Simplified diagram of radiative transfer process for signal reaching sensor reflectance $\rho$ is comprised of two terms, direct solar radiance $L_{s}$ projected onto the surface with relative orientation $\theta$ and integrated diffuse solar radiance $L_{d}$. This reflected illumination must then travel through the atmosphere, characterized by a spectral transmission $\tau$. Additionally, illumination is scattered into the sensor's field of view by the atmosphere itself, giving rise to a path radiance term denoted as $L_{p}$. A hyperspectral sensor obtains measurements at $K$ discrete, contiguous spectral bands denoted by center wavelengths $\lambda_{k}$ for $k=1, \ldots, K$. A hyperspectral measurement vector $\mathbf{L}$ for spatial row $m$ and spatial column $n$ can be modeled by the sum of three components described by $[76,16]$,

$$
\mathbf{L}[m, n]=\boldsymbol{\rho}[m, n] \odot\left(\boldsymbol{\tau} \odot \mathbf{L}_{s} \cos \theta_{m, n}+\boldsymbol{\tau} \odot \mathbf{L}_{d}\right)+\mathbf{L}_{p}
$$


where $\odot$ denotes element-wise vector product and

$$
\begin{aligned}
\mathbf{L}[m, n] & =\left[L\left[m, n, \lambda_{1}\right], \ldots, L\left[m, n, \lambda_{K}\right]\right]^{T} \\
\boldsymbol{\rho}[m, n] & =\left[\rho\left[m, n, \lambda_{1}\right], \ldots, \rho\left[m, n, \lambda_{K}\right]\right]^{T} \\
\mathbf{L}_{s} & =\left[L_{s}\left[\lambda_{1}\right], \ldots, L_{s}\left[\lambda_{K}\right]\right]^{T} \\
\mathbf{L}_{d} & =\left[L_{d}\left[\lambda_{1}\right], \ldots, L_{d}\left[\lambda_{K}\right]\right]^{T} \\
\mathbf{L}_{p} & =\left[L_{p}\left[\lambda_{1}\right], \ldots, L_{p}\left[\lambda_{K}\right]\right]^{T} \\
\boldsymbol{\tau} & =\left[\tau\left[\lambda_{1}\right], \ldots, \tau\left[\lambda_{K}\right]\right]^{T}
\end{aligned}
$$

for $m=1, \ldots, M$ where $M$ is the total number of spatial rows and $n=1, \ldots, N$ where $N$ is the total number of spatial columns in the data cube.

This model assumes all materials in the scene receive the same amount of direct and diffuse radiance. However, rarely does one encounter this ideal situation as the incident illumination varies for portions of the scene because nearby objects, such as buildings and trees, can block a fraction of the illumination. Consequently, a more complete model describing the signal incorporates spatially varying scalar terms, or shadow coefficients, to account for varying degrees of incident illumination. Let $\alpha$ represent the fraction of direct solar radiance and $\beta$ the fraction of diffuse solar radiance, both relative to a fully illuminated pixel. By replacing $\mathbf{L}_{s}$ with $\alpha[m, n] \mathbf{L}_{s}$ where $0 \leq \alpha \leq 1$, one can assume $\mathbf{L}_{s}$ to be spatially constant (i.e., not dependent on $m, n)$. Similarly, one can replace $\mathbf{L}_{d}$ with $\beta[m, n] \mathbf{L}_{d}$ where $0 \leq \beta \leq 1$ to account for situations in which a nearby object blocks a portion of the sky for the observed pixel. The $\beta$ coefficient is often referred to as "sky fraction" as it denotes the apparent percentage of sky visible to the pixel. Figure 2.2 visually depicts the radiative transfer process with incorporated shadow terms. Note this model assumes the diffuse solar 


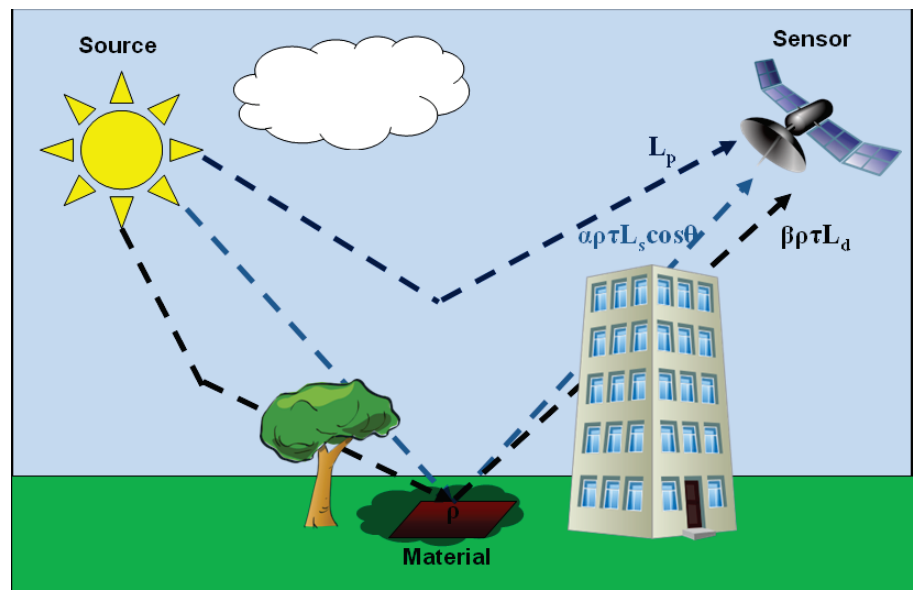

Figure 2.2: Radiative transfer process with shadow terms introduced

radiance is constant across the entire hemisphere of illumination. In reality, $\mathbf{L}_{d}$ varies spatially due to dependence on illumination angle, reflectance off nearby objects, and other additional adjacency effects. However, at this point it is assumed spatially constant assuming the deviations from this simplification are small relative to the other terms of the model. With these additional model parameters, one can describe the sensor-reaching radiance for spatial pixel $(m, n)$ as,

$$
\mathbf{L}[m, n]=\boldsymbol{\rho}[m, n] \odot\left(\alpha[m, n] \boldsymbol{\tau} \odot \mathbf{L}_{s} \cos \theta_{m, n}+\beta[m, n] \boldsymbol{\tau} \odot \boldsymbol{L}_{d}\right)+\mathbf{L}_{p}+\boldsymbol{\nu}[m, n]
$$

where $\boldsymbol{\nu}[m, n]$ is an additive random sensor noise term. Both the direct shadow coefficient $\alpha[m, n]$ and the projection term $\cos \theta_{m, n}$ will take values between 0 and 1 . Consequently, both terms are combined into a single parameter denoted by $\tilde{\alpha}[m, n]=$ $\alpha[m, n] \cos \theta_{m, n}$ and the model is rewritten as,

$$
\mathbf{L}[m, n]=\boldsymbol{\rho}[m, n] \odot\left(\tilde{\alpha}[m, n] \boldsymbol{\tau} \odot \mathbf{L}_{s}+\beta[m, n] \boldsymbol{\tau} \odot \boldsymbol{L}_{d}\right)+\mathbf{L}_{p}+\boldsymbol{\nu}[m, n]
$$

The $\tilde{\alpha}[m, n]$ parameter now provides information about the surface orientation and direct shadow condition for a particular pixel. The significance of the given model 
resides in the spatial and spectral dependencies of the parameters. For simplicity, the terms $\boldsymbol{\tau} \odot \mathbf{L}_{s}, \boldsymbol{\tau} \odot \boldsymbol{L}_{d}$, and $\mathbf{L}_{p}$ will be referred to as the "radiative transfer $(\mathrm{RT})$ terms" of the model, and $\tilde{\alpha}$ and $\beta$ will be referred to as the "shadow coefficients" of the model. While material reflectance varies both spectrally $\left(\lambda_{k}\right)$ and spatially $(m, n)$, the radiative transfer terms vary only spectrally and the shadow coefficient terms vary only spatially. While this model does not incorporate other physical phenomena caused by nearby pixels, such as adjacency effects, this model is employed due to its simplicity. A more complete physical model incorporating adjacency and nonlinear scattering effects is provided in Appendix A. The significance of the nonlinear scattering and adjacency effects is highly dependent on the degree of atmospheric turbidity. As a result, the accuracy of the linear model will depend on the atmospheric conditions of the scene being modeled.

\subsubsection{Subspace Data Model}

The physical model above can be used to formulate the change detection problem, which essentially corresponds to both a hypothesis testing problem and a parameter estimation problem. With the RT terms and shadow coefficients associated with (2.2) known with confidence at any given time, one can directly estimate material reflectance for any given pixel and perform a direct comparison for multiple data sets to determine if the reflectance has changed. For the current model, a single hyperspectral vector measurement has a total of $4 K+2$ unknown parameters associated with it ( $K$ each for the reflectance term, the transmission-direct radiance product, the transmission-diffuse radiance product, and the path radiance, and one for each of 
the shadow coefficients $\tilde{\alpha}$ and $\beta$ ). Since only $K$ spectral measurements exist, the estimation problem is infeasible. Additionally, due to the nonlinearity resulting from the product of the unknown RT terms with the reflectance, one cannnot expect to effectively estimate the reflectance separately from the RT terms. However, Chandra and Healey demonstrate that the RT terms can be effectively represented using a lower dimensional coupled linear subspace $[16,17]$. Using the atmospheric modeling tool MODTRAN (MODerate resolution atmospheric TRANsmittance and radiance code), the radiative transfer terms associated with the measurement vector $\left(\tau, \mathbf{L}_{s}, \mathbf{L}_{d}, \mathbf{L}_{p}\right)$ can be simulated for various atmospheric conditions and solar/sensor geometries $[5,6]$. Further information about MODTRAN is provided in Appendix A. By generating a set of possible vector realizations, an approximation of the corresponding subspace is developed. From this subspace, basis vectors can be determined using a singular value decomposition (SVD). Using these basis vectors, the radiative transfer terms of the model are approximated by,

$$
\begin{aligned}
& \boldsymbol{\tau} \odot \mathbf{L}_{s} \approx \sum_{i=1}^{I} \mathbf{s}_{i} \epsilon_{i}=\mathbf{S} \boldsymbol{\epsilon} \\
& \boldsymbol{\tau} \odot \mathbf{L}_{d} \approx \sum_{i=1}^{I} \mathbf{d}_{i} \epsilon_{i}=\mathbf{D} \boldsymbol{\epsilon} \\
& \mathbf{L}_{p} \approx \sum_{i=1}^{I} \mathbf{p}_{i} \epsilon_{i}=\mathbf{P} \boldsymbol{\epsilon}
\end{aligned}
$$

where $\mathbf{s}_{i}, \mathbf{d}_{i}$, and $\mathbf{p}_{i}$ for $i=1, \ldots, I$ are the basis vectors for the respective terms with basis coefficients $\epsilon_{i}$ and

$$
\begin{aligned}
& \mathbf{S}=\left[\mathbf{s}_{1}, \ldots, \mathbf{s}_{I}\right] \\
& \mathbf{D}=\left[\mathbf{d}_{1}, \ldots, \mathbf{d}_{I}\right] \\
& \mathbf{P}=\left[\mathbf{p}_{1}, \ldots, \mathbf{p}_{I}\right] \\
& \boldsymbol{\epsilon}=\left[\epsilon_{1}, \ldots, \epsilon_{I}\right]^{T}
\end{aligned}
$$


where $I$ is the subspace dimension. Note that the basis coefficients $\epsilon_{i}$ for all three terms are the same. This results from the development of the coupled-subspace as outlined in Chandra's work [16]. The transmission, direct radiance, diffuse radiance, and path radiance are all physically correlated as they depend upon the same atmospheric parameters. Consequently, the terms should not be permitted to vary independently of each other. In Chandra's work, the dimensional $I$ is determined by assessing the approximation error of a simulated measurement after projection onto the lower dimensional subspace of dimension $I[17]$. This subspace formulation greatly assists with the estimation problem by not only reducing the number of unknown parameters, but by additionally providing separability between the reflectance and RT terms by forcing the RT terms to reside in a lower dimensional subspace. Using the subspace approximation, the data model is now written as,

$$
\mathbf{L}[m, n] \approx \boldsymbol{\rho}[m, n] \odot(\tilde{\alpha}[m, n] \mathbf{S} \boldsymbol{\epsilon}+\beta[m, n] \mathbf{D} \boldsymbol{\epsilon})+\mathbf{P} \boldsymbol{\epsilon}+\boldsymbol{\nu}[m, n]
$$

for which the number of unknown parameters has been reduced to $K+I+2$ with separability existing between the RT terms and the reflectance term resulting from the subspace constraint imposed on the RT terms. The number of unknown parameters for a particular measurement vector is still greater than the number of measurements $(K)$. Consequently, estimation of the model parameters (in particular, reflectance) is still infeasible. Chandra further simplifies the model by employing a subspace model for the reflectance term thereby reducing the number of unknowns to less than $K$, creating a feasible estimation problem. However, the restriction of the reflectance vector to a particular subspace is not applied at this time to allow for model application in a diverse set of environments. 


\subsection{Change Detection Hypothesis Test}

Using the subspace data model, a hypothesis test for change detection is developed. For change detection, two measurement vectors exist for each spatial pixel $m, n$ given by,

$$
\begin{aligned}
\mathbf{L}^{(1)}[m, n] & \approx \boldsymbol{\rho}^{(1)}[m, n] \odot\left(\tilde{\alpha}^{(1)}[m, n] \mathbf{S}^{(1)} \boldsymbol{\epsilon}^{(1)}+\beta^{(1)}[m, n] \mathbf{D}^{(1)} \boldsymbol{\epsilon}^{(1)}\right) \\
+ & \mathbf{P}^{(1)} \boldsymbol{\epsilon}^{(1)}+\boldsymbol{\nu}^{(1)}[m, n] \\
\mathbf{L}^{(2)}[m, n] & \approx \boldsymbol{\rho}^{(2)}[m, n] \odot\left(\tilde{\alpha}^{(2)}[m, n] \mathbf{S}^{(2)} \boldsymbol{\epsilon}^{(2)}+\beta^{(2)}[m, n] \mathbf{D}^{(2)} \boldsymbol{\epsilon}^{(2)}\right) \\
+ & \mathbf{P}^{(2)} \boldsymbol{\epsilon}^{(2)}+\boldsymbol{\nu}^{(2)}[m, n]
\end{aligned}
$$

where the superscript $^{(t)}$ for $t=1,2$ is used to denote time- 1 or time- 2 parameters. Note that the RT subspace basis vectors are permitted to vary for time- 1 and time- 2 . If information exists about the data collection at time- 1 and time-2 (such as exact time of day, sensor altitude, etc.), the RT subspace can be further constrained to one more representative of the data set. If no such information exists, the same set of basis vectors can be used for both times, i.e.,

$$
\begin{aligned}
& \mathbf{S}^{(1)}=\mathbf{S}^{(2)}=\mathbf{S} \\
& \mathbf{D}^{(1)}=\mathbf{D}^{(2)}=\mathbf{D} \\
& \mathbf{P}^{(1)}=\mathbf{P}^{(2)}=\mathbf{P}
\end{aligned}
$$

Using the joint data model, a hypothesis test for change detection is developed.

\subsubsection{Hypothesis Test}

As discussed earlier, one is typically interested in identifying changes in material reflectance. Consequently, the hypothesis test for spatial pixel $(m, n)$ is given by,

$$
\begin{array}{ll}
H_{0}: & \boldsymbol{\rho}^{(1)}[m, n]=\boldsymbol{\rho}^{(2)}[m, n] \\
H_{1}: & \boldsymbol{\rho}^{(1)}[m, n] \neq \boldsymbol{\rho}^{(2)}[m, n]
\end{array}
$$

where $H_{0}$ is the null hypothesis of no reflectance change and $H_{1}$ is the alternative hypothesis of a change occurring. The alternative hypothesis is simply a change 
in reflectance with no differentiation given to specific types of reflectance change occurring. This type of change detection is often referred to as anomalous change detection, as one simply tries to identify any type of reflectance change rather than a specific type. For anomalous change detection, a change in the physical state of the object, such as vegetation health, that manifests itself as a change in reflectance is treated equally as a change due to the insertion or removal of an object, which is also observed as a change in spectral reflectance. Note that each pixel is tested independently from the rest. In certain cases, one would expect change targets to occupy a group of neighboring pixels, in which case one would considering applying the hypothesis test to a group of pixels jointly. Here, the pixels are tested individually to allow for detection of single-pixel and sub-pixel change targets that can exist when utilizing airborne sensors with poor spatial resolution.

Based upon the statistical model and assumptions made for the data, different likelihood ratio tests can be developed. Since no decision costs are available and no prior information about the likelihood of each hypothesis exists, Neyman-Pearson detectors are examined [45]. The basic likelihood ratio associated with the problem is given by,

$$
D\left(\mathbf{L}^{(1)}[m, n], \mathbf{L}^{(2)}[m, n]\right)=\frac{p\left(\mathbf{L}^{(1)}[m, n], \mathbf{L}^{(2)}[m, n] \mid H_{1}\right)}{p\left(\mathbf{L}^{(1)}[m, n], \mathbf{L}^{(2)}[m, n] \mid H_{0}\right)}
$$

where $p\left(\mathbf{L}^{(1)}[m, n], \mathbf{L}^{(2)}[m, n] \mid H_{q}\right)$ is the joint probability density function (pdf) of the data under hypothesis $H_{q}$. The associated likelihood ratio test (LRT) is given by,

$$
D\left(\mathbf{L}^{(1)}[m, n], \mathbf{L}^{(2)}[m, n]\right) \underset{H_{0}}{\stackrel{H_{1}}{\gtrless}} \gamma
$$

where $\gamma$ is a threshold set to maximize probability of detection $P_{d}$ for a certain probability of false alarm $P_{f}$. 
From here, several assumptions are made about the random noise components $\boldsymbol{\nu}^{(1)}[m, n]$ and $\boldsymbol{\nu}^{(2)}[m, n]$ to derive a specific form for $D$. The noise components in this model represent only sensor noise rather than background clutter plus sensor noise, which requires a more general noise model. The sensor noise components are assumed to be zero-mean, normally distributed and conditionally independent in the spectral, spatial, and temporal dimensions given the signal level. Hyperspectral sensors typically utilize photon detectors, such as CCD cameras, to generate measurements. The variability in measurements generated from this sensing modality results from photon noise and electronics/read noise [38, 40, 76, 46]. The photon noise has a variance directly dependent on the number of photons detected, or equivalently, the amplitude of the measured signal. As a result, the sensor noise is dependent on signal level and, consequently, will vary both spatially and spectrally. While the signal-dependent noise component results from a Poisson random process, the number of photons is typically large, such that a Gaussian assumption is valid. The electronics/read noise can be modeled as Gaussian as well $[38,40,76,46]$. Further development and discussion about the noise and noise estimation is provided in Chapter 3. The sensor noise is also assumed to be independent pixel to pixel, which is a reasonable assumption if no significant defects exist in the camera. This independence produces data noise that is independent spatially, spectrally and temporally. For certain sensors, the signal-independent noise source may dominate the signal-dependent noise, in which case the spatial dependence of the noise can be ignored. Under these assumptions, 
the noise covariance is given by,

$$
E\left\{\left(\boldsymbol{\nu}^{\left(t_{1}\right)}\left[m_{1}, n_{1}\right]\right)\left(\boldsymbol{\nu}^{\left(t_{2}\right)}\left[m_{2}, n_{2}\right]\right)^{T}\right\}=\left\{\begin{array}{cc}
m_{1} & \neq m_{2} \\
\mathbf{0}_{\mathbf{K}}, & n_{1} \neq n_{2} \\
& t_{1} \neq t_{2} \\
& m_{1}=m_{2}=m \\
\boldsymbol{\Gamma}^{(t)}[m, n] & n_{1}=n_{2}=n \\
t_{1}=t_{2}=t
\end{array}\right.
$$

where $\mathbf{0}_{\mathbf{K}}$ is a $K \times K$ matrix of zeros and

$$
\Gamma^{(t)}[m, n]=\operatorname{diag}\left\{\left(\sigma^{2}\right)^{(t)}\left[m, n, \lambda_{1}\right], \ldots,\left(\sigma^{2}\right)^{(t)}\left[m, n, \lambda_{K}\right]\right\}
$$

where $\operatorname{diag}\{\cdot\}$ indicates a diagonal matrix with the given entries along the diagonal. While a diagonal covariance matrix is assumed here, a more general non-diagonal covariance can be used as well. Under the temporal independence assumption, the measurements $\mathbf{L}^{(1)}$ and $\mathbf{L}^{(2)}$ are independent and normally distributed. Consequently, the joint distribution will simply be the product of the marginal distributions, i.e.,

$$
p\left(\mathbf{L}^{(1)}[m, n], \mathbf{L}^{(2)}[m, n] \mid H_{q}\right)=p\left(\mathbf{L}^{(1)}[m, n] \mid H_{q}\right) p\left(\mathbf{L}^{(2)}[m, n] \mid H_{q}\right)
$$

for $q=0,1$. The likelihood ratio can be rewritten as,

$$
D\left(\mathbf{L}^{(1)}[m, n], \mathbf{L}^{(2)}[m, n]\right)=\frac{p\left(\mathbf{L}^{(1)}[m, n] \mid H_{1}\right) p\left(\mathbf{L}^{(2)}[m, n] \mid H_{1}\right)}{p\left(\mathbf{L}^{(1)}[m, n] \mid H_{0}\right) p\left(\mathbf{L}^{(2)}[m, n] \mid H_{0}\right)}
$$

With the additional assumption of zero-mean, normally-distributed noise, the measurements will be normally distributed under both hypotheses as,

$$
\mathbf{L}^{(t)}[m, n] \mid H_{q} \sim \mathcal{N}\left(\boldsymbol{\mu}_{q}^{(t)}[m, n], \boldsymbol{\Gamma}_{q}^{(t)}[m, n]\right)
$$

where $\boldsymbol{\mu}_{q}^{(t)}[m, n]$ and $\boldsymbol{\Gamma}_{q}^{(t)}[m, n]$ are the mean vector and noise covariance for time $t$ under hypothesis $q$ respectively. From (2.5), the mean vector under $H_{1}$ is given by,

$$
\boldsymbol{\mu}_{1}^{(t)}[m, n]=\boldsymbol{\rho}^{(t)}[m, n] \odot\left(\tilde{\alpha}^{(t)}[m, n] \mathbf{S}^{(t)} \boldsymbol{\epsilon}^{(t)}+\beta^{(t)}[m, n] \mathbf{D}^{(t)} \boldsymbol{\epsilon}^{(t)}\right)+\mathbf{P}^{(t)} \boldsymbol{\epsilon}^{(t)}
$$


Consequently, the difference between the mean vectors for $t=1$ and $t=2$ for each spatial pixel $(m, n)$ depends upon both the change in reflectance (for which prior knowledge may not exist) and the change in radiative transfer parameters. However, under $H_{0}$ the reflectance does not change, i.e., $\boldsymbol{\rho}^{(1)}[m, n]=\boldsymbol{\rho}^{(2)}[m, n]=\boldsymbol{\rho}[m, n]$ and the mean vectors simplify to,

$$
\boldsymbol{\mu}_{0}^{(t)}[m, n]=\boldsymbol{\rho}[m, n] \odot\left(\tilde{\alpha}^{(t)}[m, n] \mathbf{S}^{(t)} \boldsymbol{\epsilon}^{(t)}+\beta^{(t)}[m, n] \mathbf{D}^{(t)} \boldsymbol{\epsilon}^{(t)}\right)+\mathbf{P}^{(t)} \boldsymbol{\epsilon}^{(t)}
$$

for $t=1,2$.

\subsubsection{Generalized Likelihood Ratio Test}

The development above assumes the data models are completely known. However, in general, the parameters of the data model given in (2.5) which determine the mean vectors are unknown along with the covariance matrices $\Gamma_{q}^{(t)}[m, n]$, thereby producing a composite hypothesis testing problem. Consequently, a generalized likelihood ratio test (GLRT) is developed in which the unknown parameters are replaced with their MLEs. In general, sensor noise estimation for hyperspectral data is a complex problem with more general applications than use for change detection. As a result, this problem is examined separately in Chapter 3 and techniques are discussed to obtain

an estimate of the noise covariance $\boldsymbol{\Gamma}_{q}^{(t)}[m, n]$ using knowledge about the sensor and calibration processes or estimating the noise directly from the imagery. For now, an estimate of the noise covariance is assumed to exist allowing for a simplified GLRT derivation. In many situations, it may not be possible to develop a signal-dependent noise model for the data. In these cases, the user can simply assume white noise where any resulting performance degradation will be dependent on how inaccurate this assumption is for the particular data set being tested. Assuming the signal-dependent 
noise model, the GLRT for the problem is given by,

$$
D_{G}\left(\mathbf{L}^{(1)}[m, n], \mathbf{L}^{(2)}[m, n]\right)=\frac{p\left(\mathbf{L}^{(1)}[m, n] \mid \hat{\boldsymbol{\mu}}_{1}^{(1)}[m, n], H_{1}\right) p\left(\mathbf{L}^{(2)}[m, n] \mid \hat{\boldsymbol{\mu}}_{1}^{(2)}[m, n], H_{1}\right)}{p\left(\mathbf{L}^{(1)}[m, n] \mid \hat{\boldsymbol{\mu}}_{0}^{(1)}[m, n], H_{0}\right) p\left(\mathbf{L}^{(2)}[m, n] \mid \hat{\boldsymbol{\mu}}_{0}^{(2)}[m, n], H_{0}\right)}
$$

where $\hat{\boldsymbol{\mu}}_{q}^{(t)}[m, n]$ is the MLE of the mean vector at time $t$ under hypothesis $q$ as defined in (2.12) and (2.13). In anomalous change detection, one seeks to detect any change in reflectance occurring from time-1 to time-2. Consequently, no specific information exists about the type of reflectance change and corresponding change in mean vectors given in (2.12). From estimation theory, when no additional information exists for the mean parameter in a data model, the resulting MLE of the mean from a single measurement is simply the data measurement itself, i.e.,

$$
\hat{\boldsymbol{\mu}}_{1}^{(t)}[m, n]=\mathbf{L}^{(t)}[m, n]
$$

for $t=1,2$. Under these assumptions, the exponential term in the numerator vanishes. Using the log-GLRT with Gaussian likelihood functions results in,

$$
\begin{aligned}
& \tilde{D}_{G}\left(\mathbf{L}^{(1)}[m, n], \mathbf{L}^{(2)}[m, n]\right)= \\
& \left(\mathbf{L}^{(1)}[m, n]-\hat{\boldsymbol{\mu}}_{0}^{(1)}[m, n]\right)^{T}\left(\boldsymbol{\Gamma}_{0}^{(1)}[m, n]\right)^{-1}\left(\mathbf{L}^{(1)}[m, n]-\hat{\boldsymbol{\mu}}_{0}^{(1)}[m, n]\right)^{T} \\
& +\left(\mathbf{L}^{(2)}[m, n]-\hat{\boldsymbol{\mu}}_{0}^{(2)}[m, n]\right)^{T}\left(\boldsymbol{\Gamma}_{0}^{(2)}[m, n]\right)^{-1}\left(\mathbf{L}^{(2)}[m, n]-\hat{\boldsymbol{\mu}}_{0}^{(2)}[m, n]\right)^{T} \\
& +C_{0,1}[m, n]
\end{aligned}
$$

where

$$
C_{0,1}[m, n]=\frac{1}{2} \ln \left(\frac{\left|\boldsymbol{\Gamma}_{0}^{(1)}[m, n]\right|\left|\boldsymbol{\Gamma}_{0}^{(2)}[m, n]\right|}{\left|\boldsymbol{\Gamma}_{1}^{(1)}[m, n]\right|\left|\boldsymbol{\Gamma}_{1}^{(2)}[m, n]\right|}\right) .
$$

Due to the signal-dependent noise model, the noise covariance for both hypotheses will be the same as the estimate is obtained using the same measurement. Consequently, $C_{0,1}[m, n]=0$, which results in a test statistic achieving a constant false alarm rate for a given threshold. 
The remaining issue is obtaining the MLEs $\hat{\boldsymbol{\mu}}_{0}^{(1)}[m, n]$ and $\hat{\boldsymbol{\mu}}_{0}^{(2)}[m, n]$. The GLRT is applied to each pixel $m, n$ in the scene separately. Additionally, the mean vectors defined in (2.13) vary for each pixel because the reflectance and shadow coefficient terms are dependent on $m, n$. However, the basis coefficients $\boldsymbol{\epsilon}$ do not depend on $m, n$. As a result, coupled information exists among all $M N$ data measurements and this information can be exploited. The data model parameters are estimated for the entire joint data set to make use of this coupled information. The new MLE problem is given by,

$$
\hat{\mathbf{x}}=\underset{\mathbf{x}}{\arg \max } p\left(\tilde{\mathbf{L}}^{(1)}, \tilde{\mathbf{L}}^{(2)} \mid \mathbf{x}, H_{0}\right)
$$

where $\tilde{\mathbf{L}}^{(t)}$ is the entire data cube at time $t$ and $\mathbf{x}$ is the unknown parameter vector for all the data given by,

$$
\mathbf{x}=\left[\mathbf{x}_{\rho}^{T}, \mathbf{x}_{s}^{T}, \mathbf{x}_{\epsilon}^{T}\right]^{T}
$$

with

$$
\begin{aligned}
\mathbf{x}_{\rho} & =\left[\boldsymbol{\rho}^{T}[1,1], \ldots, \boldsymbol{\rho}^{T}[M, N]\right]^{T} \\
\mathbf{x}_{s} & =\left[\tilde{\alpha}^{(1)}[1,1], \ldots, \tilde{\alpha}^{(1)}[M, N], \tilde{\alpha}^{(2)}[1,1], \ldots, \tilde{\alpha}^{(2)}[M, N],\right. \\
& \left.\beta^{(1)}[1,1], \ldots, \beta^{(1)}[M, N], \beta^{(2)}[1,1], \ldots, \beta^{(2)}[M, N]\right]^{T} \\
\mathbf{x}_{\epsilon} & =\left[\left(\boldsymbol{\epsilon}^{(1)}\right)^{T},\left(\boldsymbol{\epsilon}^{(2)}\right)^{T}\right]^{T}
\end{aligned}
$$

which contain the reflectance vectors for all pixels, the shadow coefficients for all pixels at time- 1 and time- 2 , and the RT basis coefficients at time- 1 and time-2. From the noise independence assumptions, the problem becomes

$$
\begin{aligned}
\hat{\mathbf{x}} & =\underset{\mathbf{x}}{\arg \max } \prod_{m, n} p\left(\mathbf{L}^{(1)}[m, n], \mathbf{L}^{(2)}[m, n] \mid \mathbf{x}, H_{0}\right) \\
& =\underset{\mathbf{x}}{\arg \max } \prod_{m, n} p\left(\mathbf{L}^{(1)}[m, n] \mid \mathbf{x}, H_{0}\right) p\left(\mathbf{L}^{(2)}[m, n] \mid \mathbf{x}, H_{0}\right)
\end{aligned}
$$


Using the Gaussian noise assumptions, an equivalent problem is given by,

$$
\hat{\mathbf{x}}=\underset{\mathbf{x}}{\arg \min } \sum_{m, n} D^{(1)}[m, n \mid \mathbf{x}]+D^{(2)}[m, n \mid \mathbf{x}]
$$

with

$$
D^{(t)}[m, n \mid \mathbf{x}]=\left(\mathbf{L}^{(t)}[m, n]-\boldsymbol{\mu}_{0}^{(t)}[m, n \mid \mathbf{x}]\right)^{T}\left(\boldsymbol{\Gamma}^{(t)}[m, n]\right)^{-1}\left(\mathbf{L}^{(t)}[m, n]-\boldsymbol{\mu}_{0}^{(t)}[m, n \mid \mathbf{x}]\right)^{T}
$$

where $\boldsymbol{\mu}_{0}^{(t)}[m \mid \mathbf{x}]$ is the mean vector at time $t$ for pixel $(m, n)$ which is dependent on the model parameters given in $\mathbf{x}$. The summation term in (2.22) is essentially the GLRT given in (2.16). Consequently, a nonlinear estimation problem exists for which the residual estimation error can be used to make a decision on the occurrence of a change.

Note that several ambiguities exist when using the data model given in (2.5) that result in undetectable changes under the current formulation. In particular, the model cannot differentiate between a zero reflectance object $\boldsymbol{\rho}=0$ and total shadow $\alpha=\beta=0$. Consequently, if a zero reflectance object appeared/disappeared from time-1 to time-2, this object cannot be detected. Additionally, the model cannot differentiate a scalar change in reflectance from time-1 to time-2. Since the shadow coefficients are spatially-varying scalar constants, these terms can account for scalar changes in reflectance. Overall, these ambiguities are not considered significant as these scenarios very rarely occur in practice and, more importantly, correspond to uninteresting changes.

\subsection{Optimization Problem}

The development above has resulted in a nonlinear estimation problem for which the unknown parameters are constrained to a known subset. In parameter estimation 
problems, a cost function $f(\mathbf{x})$ is minimized, which is dependent on the unknown parameters $\mathbf{x}$ in order to determine the state estimates. In this case, the cost function is given by the summation in (2.22). Optimization theory is examined for techniques on how to solve this constrained nonlinear estimation problem.

\subsubsection{Cost Function and Constraints}

From (2.22), the cost function of the estimation problem is given by,

$$
f(\mathbf{x})=\sum_{m, n} D^{(1)}[m, n \mid \mathbf{x}]+D^{(2)}[m, n \mid \mathbf{x}]
$$

The length of the parameter vector is $K M N+2 I+4 M N$ with $2 K M N$ associated measurements. Note that $K>4$ is required in order for the estimation problem to be feasible. As a result, this change detection method is only applicable to multispectral data with greater than 4 spectral bands. The possible estimates of the unknown parameters in $\mathbf{x}$ are limited to a specific subset of real values. Physically, reflectance of a material cannot be less than 0 or greater than 1 . The shadow coefficients are bounded in the same way. One could simply place a lower bound of 0 for radiance values with no upper bound. However, the RT basis coefficients can be further constrained using a linear inequality constraint developed by utilizing the MODTRAN realizations described in Section 2.2.2. These simulations can be used to assign lower and upper inequality bounds on the possible radiance values for each of the RT terms by determining the minimum and maximum radiance values at each wavelength for the three separate model terms for $t=1,2$ and designate these as $\left(\mathbf{L}_{s}^{l b}, \mathbf{L}_{s}^{u b}\right),\left(\mathbf{L}_{d}^{l b}\right.$, $\left.\mathbf{L}_{d}^{u b}\right)$ and $\left(\mathbf{L}_{p}^{l b}, \mathbf{L}_{p}^{u b}\right)$. An additional bound constraint can be placed on the RT basis coefficients themselves to enforce physically sensible curves for the radiative transfer terms. In general, the basis vectors are ordered in such a way that one expects the 
magnitude of the leading basis coefficient $\epsilon[1]$ to be greater than the remaining coefficients. The magnitude of the trailing basis coefficients should decrease in general and eventually become small. One can impose these constraints by using the MODTRAN realizations to compute the respective basis coefficients for each realization using the derived basis vectors. The lower and upper bound for the basis coefficients are determined and designated as $\boldsymbol{\epsilon}^{l b}$ and $\boldsymbol{\epsilon}^{u b}$ respectively. Note the linear inequality and radiative transfer basis coefficient bound constraints can be determined separately for time- 1 and time- 2 data if prior knowledge exists about the data collection at each time, such as time of day, sensor altitude, etc. These constraints are summarized by,

$$
\begin{array}{rlrl}
0 & \preceq \boldsymbol{\rho}[\boldsymbol{m}, \boldsymbol{n}] \preceq 1 & & m=1, \ldots, M, n=1, \ldots, N \\
0 & \leq \tilde{\alpha}^{(t)}[m, n] \leq 1 & & m=1, \ldots, M, n=1, \ldots, N, t=1,2 \\
0 & \leq \beta^{(t)}[m, n] \leq 1 & & m=1, \ldots, M, n=1, \ldots, N, t=1,2 \\
\mathbf{L}_{s}^{l b(t)} \preceq \mathbf{S}^{(t)} \boldsymbol{\epsilon}^{(t)} \preceq \mathbf{L}_{s}^{u b(t)} & t=1,2 \\
\mathbf{L}_{d}^{l b(t)} \preceq \mathbf{D}^{(t)} \boldsymbol{\epsilon}^{(t)} \preceq \mathbf{L}_{d}^{u b(t)} & t=1,2 \\
\mathbf{L}_{p}^{l b(t)} \preceq \mathbf{P}^{(t)} \boldsymbol{\epsilon}^{(t)} \preceq \mathbf{L}_{p}^{u b(t)} & t=1,2 \\
\boldsymbol{\epsilon}^{l b(t)} \preceq \boldsymbol{\epsilon}^{(t)} \preceq \boldsymbol{\epsilon}^{u b(t)} & t=1,2
\end{array}
$$

where $\preceq$ is used to denote element-wise inequality. Under this formulation, the optimal parameter estimates are obtained via,

$$
\mathbf{x}^{*}=\underset{\mathbf{x} \in X}{\arg \min } f(\mathbf{x})
$$

where $X \subseteq \mathbb{R}^{K M N+2 I+4 M N}$ is the subset of real vectors satisfying the constraint set $(2.25)$.

\subsubsection{Optimization Algorithm}

Various methods exist in the literature for solving constrained optimization problems such as the one above [9]. Implementation presents a significant challenge due to the enormous number of unknown parameters (degrees of freedom) and the nonlinearity of the problem. For example, a relatively small hyperspectral data cube can 
be of spatial size $M=200$ rows, $N=200$ columns and $K=100$ spectral bands. The total optimization problem would possess 8,000,000 measurements with 4,160,020 unknown parameters assuming $I=10$. Due to the size and complexity of this problem, optimizing the full problem ultimately proves an intractable task requiring massive computing resources. Additionally, the complexity of the cost function may present convergence issues if relatively good initial estimates are not available. As a result, the problem is broken into several smaller estimation problems using a technique often referred to as alternating optimization (AO) [10]. Essentially, this approach operates by treating a certain number of unknown parameters as known while estimating the remaining parameters. After these parameters are estimated, they are treated as known and the first set of parameters are estimated. This alternating technique can continue for a fixed number of iterations or until convergence criteria are met.

The optimization problem (2.26) can be decomposed into three smaller and simpler estimation problems. Here the parameter vector $\mathbf{x}$ is split into the three sub-vectors $\mathbf{x}_{\rho}, \mathbf{x}_{s}$ and $\mathbf{x}_{\epsilon}$ as defined in (2.20). In this way, the estimation of the reflectance, shadow coefficients, and RT basis coefficients are separated. The idea behind AO is to decompose the problem by optimizing over a limited number of dimensions of the cost function at any given step. This formulation is applied under the assumption that convergence to the global minimum (assuming existence) of the cost function can be reached in this alternating fashion, which is true when the cost function is convex. In practice, the user may encounter difficulties with local minima present. Consequently, good initialization is important to help ensure the cost function is convex in the immediate neighborhood. Figure 2.3 displays a simple flow chart illustrating the AO process. A couple of important consequences arise from this optimization 


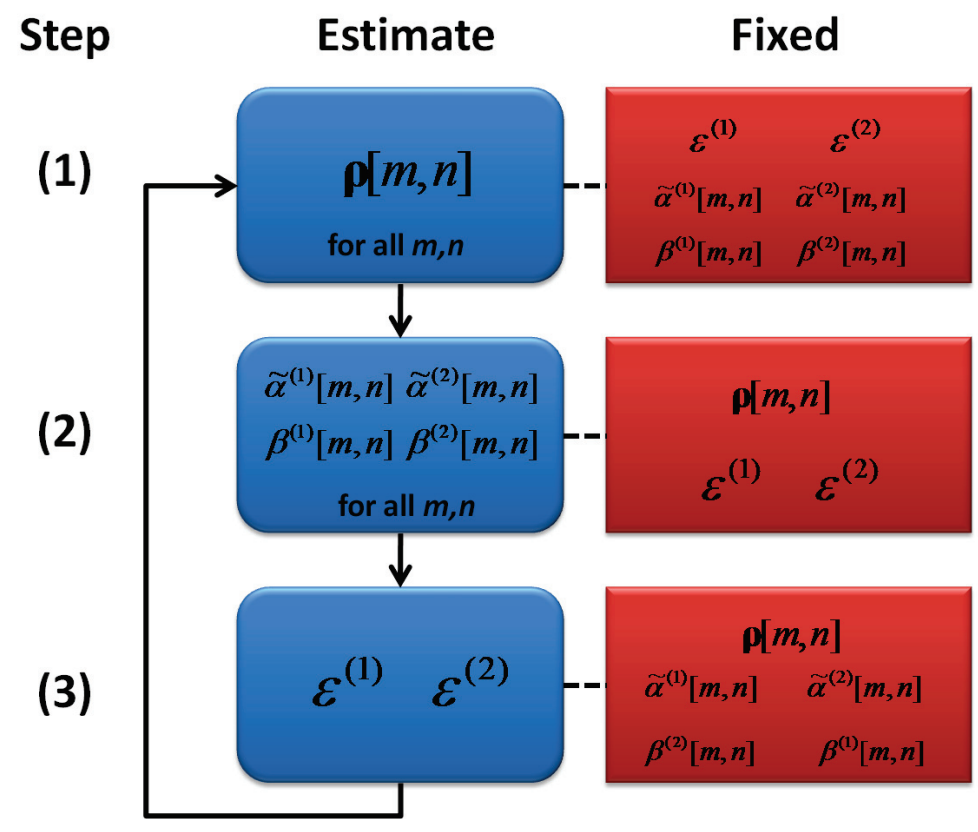

Figure 2.3: Flow chart for alternating optimization applied for model-based change detection

simplification. First, from investigation of the data model given in (2.5), when two of the three parameter sub-vectors above are known, the model is linear with respect to the third unknown parameter sub-vector. The linearity of the data model coupled with the linear constraints and cost function discussed above results in a simple quadratic programming problem for each of the three sub-vectors. Second, the estimation problem for the reflectance vectors and the shadow coefficients is decoupled for each pixel meaning that the reflectance vector or shadow coefficients associated with pixel $(m, n)$ are independent of the estimates for all the other pixels. While future work may choose to incorporate spatial dependence of these parameters, in this work they are treated as independent. The significance of this decoupling resides in reducing complexity and increasing the speed of implementation. The specific quadratic 
programming method implemented depends on the type of constraint specified for the problem. The RT basis coefficient optimization problem has linear inequality constraints. Consequently, the active set method described in Gill [35] is utilized. The shadow coefficient and reflectance vector problems have only bound constraints. Consequently, a subspace trust-region method based on the interior-reflective Newton method described in Coleman [20] is implemented. Each of the sub-problems is examined here to provide further detail.

\section{Reflectance Estimation Step}

The first step in the AO process is estimation of the reflectance vectors $\rho[m, n]$. With the RT basis coefficients and shadow coefficients treated as known, the change data model (2.5) can be rewritten as

$$
\begin{aligned}
& \mathbf{L}^{(1)}[m, n]=\mathbf{A}_{\rho}^{(1)}[m, n] \boldsymbol{\rho}[m, n]+\mathbf{b}^{(1)} \\
& \mathbf{L}^{(2)}[m, n]=\mathbf{A}_{\rho}^{(2)}[m, n] \boldsymbol{\rho}[m, n]+\mathbf{b}^{(2)}
\end{aligned}
$$

where

$$
\begin{aligned}
& \mathbf{A}_{\rho}^{(t)}[m, n]=\operatorname{diag}\left(\tilde{\alpha}^{(t)}[m, n] \mathbf{D} \boldsymbol{\epsilon}^{(t)}+\beta^{(t)}[m, n] \mathbf{S} \boldsymbol{\epsilon}^{(t)}\right), t=1,2 \\
& \mathbf{b}^{(t)}=\mathbf{P} \boldsymbol{\epsilon}^{(t)}, t=1,2
\end{aligned}
$$

The sum squared-error metric for this step using $\mathbf{x}_{\rho}[m, n]=\boldsymbol{\rho}[m, n]$ simplifies to,

$$
\begin{aligned}
f\left[\mathbf{x}_{\rho}[m, n]\right]=\left(\tilde{\mathbf{L}}_{c}[m, n]-\mathbf{A}_{\rho}[m, n] \mathbf{x}_{\rho}[m, n]\right)^{T}\left(\tilde{\mathbf{L}}_{c}[m, n]-\mathbf{A}_{\rho}[m, n] \mathbf{x}[m, n]\right) \\
=\tilde{\mathbf{L}}_{c}^{T}[m, n] \tilde{\mathbf{L}}_{c}[m, n]+\mathbf{x}_{\rho}^{T}[m, n] \mathbf{A}_{\rho}^{T}[m, n] \mathbf{A}_{\rho}[m, n] \mathbf{x}_{\rho}[m, n] \\
\quad-2 \tilde{\mathbf{L}}_{c}^{T}[m, n] \mathbf{A}_{\rho}[m, n] \mathbf{x}[m, n]
\end{aligned}
$$

where

$$
\mathbf{A}_{\rho}[m, n]=\left[\begin{array}{c}
\mathbf{A}_{\rho}^{(1)}[m, n] \\
\mathbf{A}_{\rho}^{(2)}[m, n]
\end{array}\right], \quad \tilde{\mathbf{L}}_{c}[m, n]=\left[\begin{array}{c}
\mathbf{L}^{(1)}[m, n] \\
\mathbf{L}^{(2)}[m, n]
\end{array}\right]-\left[\begin{array}{c}
\mathbf{b}^{(1)} \\
\mathbf{b}^{(2)}
\end{array}\right]
$$


The cost function for each pixel takes the form of a typical quadratic program given by,

$$
f\left[\mathbf{x}_{\rho}[m, n]\right]=\frac{1}{2} \mathbf{x}_{\rho}^{T}[m, n] \mathbf{G}_{\rho}[m, n] \mathbf{x}_{\rho}[m, n]+\mathbf{c}_{\rho}^{T}[m, n] \mathbf{x}_{\rho}[m, n]
$$

where

$$
\begin{aligned}
& \mathbf{G}_{\rho}[m, n]=2 \mathbf{A}_{\rho}^{T}[m, n] \mathbf{A}_{\rho}[m, n] \\
& \mathbf{c}_{\rho}[m, n]=-2 \mathbf{A}_{\rho}^{T}[m, n] \tilde{\mathbf{L}}_{c}[m, n]
\end{aligned}
$$

for which standard algorithms exist. Since $\mathbf{A}_{\rho}^{(1)}[m, n]$ and $\mathbf{A}_{\rho}^{(2)}[m, n]$ are diagonal with non-negative entries, the matrix $\mathbf{G}_{\rho}[m, n]$ will be diagonal with non-negative entries. Consequently, the matrix is positive semi-definite (PSD) and a global minimum for the optimization problem exists.

\section{Shadow Coefficient Estimation Step}

For estimation of the shadow coefficients, the measurement model can be rewritten as,

$$
\begin{aligned}
\mathbf{L}^{(1)}[m, n] & =\mathbf{A}_{s}^{(1)}[m, n]\left[\begin{array}{l}
\tilde{\alpha}^{(1)}[m, n] \\
\beta^{(1)}[m, n]
\end{array}\right]+\mathbf{b}^{(1)} \\
\mathbf{L}^{(2)}[m, n] & =\mathbf{A}_{s}^{(2)}[m, n]\left[\begin{array}{l}
\tilde{\alpha}^{(2)}[m, n] \\
\beta^{(2)}[m, n]
\end{array}\right]+\mathbf{b}^{(2)}
\end{aligned}
$$

where

$$
\mathbf{A}_{s}^{(t)}[m, n]=\left[\boldsymbol{\rho}[m, n] \odot \mathbf{D} \boldsymbol{\epsilon}^{(t)} \quad \boldsymbol{\rho}[m, n] \odot \mathbf{S} \boldsymbol{\epsilon}^{(t)}\right], t=1,2
$$

The sum squared-error metric for this step using,

$$
\mathbf{x}_{s}[m, n]=\left[\tilde{\alpha}^{(1)}[m, n] \beta^{(1)}[m, n] \tilde{\alpha}^{(2)}[m, n] \beta^{(2)}[m, n]\right]^{T}
$$

simplifies to,

$$
\begin{gathered}
f\left[\mathbf{x}_{s}[m, n]\right]=\tilde{\mathbf{L}}_{c}^{T}[m, n] \tilde{\mathbf{L}}_{c}[m, n]+\mathbf{x}_{s}^{T}[m, n] \mathbf{A}_{s}^{T}[m, n] \mathbf{A}_{s}[m, n] \mathbf{x}_{s}[m, n] \\
\quad-2 \tilde{\mathbf{L}}_{c}^{T}[m, n] \mathbf{A}_{s}[m, n] \mathbf{x}_{s}[m, n]
\end{gathered}
$$

where

$$
\mathbf{A}_{s}[m, n]=\left[\begin{array}{cc}
\mathbf{A}_{s}^{(1)}[m, n] & \mathbf{0} \\
\mathbf{0} & \mathbf{A}_{s}^{(2)}[m, n]
\end{array}\right]
$$


The cost function for each pixel takes the form of a typical quadratic program given by,

$$
f\left[\mathbf{x}_{s}[m, n]\right]=\frac{1}{2} \mathbf{x}_{s}^{T}[m, n] \mathbf{G}_{s}[m, n] \mathbf{x}_{s}[m, n]+\mathbf{c}_{s}^{T}[m, n] \mathbf{x}_{s}[m, n]
$$

where

$$
\begin{aligned}
& \mathbf{G}_{s}[m, n]=2 \mathbf{A}_{s}^{T}[m, n] \mathbf{A}_{s}[m, n] \\
& \mathbf{c}_{s}[m, n]=-2 \mathbf{A}_{s}^{T}[m] \tilde{\mathbf{L}}_{c}[m, n]
\end{aligned}
$$

The question remains of whether $\mathbf{G}[m, n]$ is PSD. Let

$$
\begin{aligned}
\mathbf{v}_{d}^{(t)}[m, n] & =\boldsymbol{\rho}[m, n] \odot \mathbf{D} \boldsymbol{\epsilon}^{(t)} \\
\mathbf{v}_{s}^{(t)}[m, n] & =\boldsymbol{\rho}[m, n] \odot \mathbf{S} \boldsymbol{\epsilon}^{(t)}
\end{aligned}
$$

Then,

$$
\left(\mathbf{A}_{s}^{(t)}[m, n]\right)^{T} \mathbf{A}_{s}^{(t)}[m, n]=\left[\begin{array}{cc}
\left(\mathbf{v}_{d}^{(t)}[m, n]\right)^{T} \mathbf{v}_{d}^{(t)}[m, n] & \left(\mathbf{v}_{d}^{(t)}[m, n]\right)^{T} \mathbf{v}_{s}^{(t)}[m, n] \\
\left(\mathbf{v}_{d}^{(t)}[m, n]\right)^{T} \mathbf{v}_{s}^{(t)}[m, n] & \left(\mathbf{v}_{s}^{(t)}[m, n]\right)^{T} \mathbf{v}_{s}^{(t)}[m, n]
\end{array}\right]
$$

which has a determinant,

$$
\operatorname{det}\left\{\left(\mathbf{A}_{s}^{(t)}[m, n]\right)^{T} \mathbf{A}_{s}^{(t)}[m, n]\right\}=\left\|\mathbf{v}_{d}^{(t)}[m, n]\right\|\left\|^{2}\right\| \mathbf{v}_{s}^{(t)}[m, n]\|\|^{2}-\left\langle\mathbf{v}_{d}^{(t)}[m, n], \mathbf{v}_{s}^{(t)}[m, n]\right\rangle^{2}
$$

where $\langle\cdot, \cdot\rangle$ is used to denote inner product. From the Cauchy-Schwartz inequality,

$$
\left.\left\|\mathbf{v}_{d}^{(t)}[m, n]\right\|\right|^{2}\left\|\mathbf{v}_{s}^{(t)}[m, n]\right\|^{2} \geq\left\langle\mathbf{v}_{d}^{(t)}[m, n], \mathbf{v}_{s}^{(t)}[m, n]\right\rangle^{2}
$$

Consequently,

$$
\operatorname{det}\left\{\left(\mathbf{A}_{s}^{(t)}[m, n]\right)^{T} \mathbf{A}_{s}^{(t)}[m, n]\right\} \geq 0
$$

For the quadratic matrix $\mathbf{G}_{s}[m, n]$,

$$
\begin{aligned}
& \mathbf{G}_{s}[m, n]=2 \mathbf{A}_{s}^{T}[m, n] \mathbf{A}_{s}[m, n] \\
& =2\left[\begin{array}{cc}
\left(\mathbf{A}_{s}^{(1)}[m, n]\right)^{T} \mathbf{A}_{s}^{(1)}[m, n] & \mathbf{0} \\
\mathbf{0} & \left(\mathbf{A}_{s}^{(2)}[m, n]\right)^{T} \mathbf{A}_{s}^{(2)}[m, n]
\end{array}\right]
\end{aligned}
$$

Since the principal minors of $\mathbf{G}_{s}[m, n]$ are all non-negative, the matrix is positive semi-definite. 


\section{RT Basis Coefficient Estimation}

For estimation of the RT basis coefficients, the measurement model can be rewritten as,

$$
\begin{aligned}
& \mathbf{L}^{(1)}[m, n]=\mathbf{A}_{\epsilon}^{(1)}[m, n] \boldsymbol{\epsilon}^{(1)} \\
& \mathbf{L}^{(2)}[m, n]=\mathbf{A}_{\epsilon}^{(2)}[m, n] \boldsymbol{\epsilon}^{(2)}
\end{aligned}
$$

where

$$
\begin{aligned}
\mathbf{A}_{\epsilon}^{(t)}[m, n]=\left[\boldsymbol{\rho}[m, n] \odot\left(\tilde{\alpha}^{(t)}[m, n] \mathbf{d}_{1}+\beta^{(t)}[m, n] \mathbf{s}_{1}\right)+\mathbf{p}_{1}, \ldots\right. \\
\left.\quad \boldsymbol{\rho}[m, n] \odot\left(\tilde{\alpha}^{(t)}[m, n] \mathbf{d}_{I}+\beta^{(t)}[m, n] \mathbf{s}_{I}\right)+\mathbf{p}_{I}\right], t=1,2 \\
=\left[\mathbf{v}_{1}^{(t)}[m, n], \ldots, \mathbf{v}_{I}^{(t)}[m, n]\right]
\end{aligned}
$$

where

$$
\mathbf{v}_{i}^{(t)}[m, n]=\boldsymbol{\rho}[m, n] \odot\left(\tilde{\alpha}^{(t)}[m, n] \mathbf{d}_{i}+\beta^{(t)}[m, n] \mathbf{s}_{i}\right)+\mathbf{p}_{i}, t=1,2
$$

The optimization with respect to the RT basis coefficients is not decoupled for each pixel since the assumption is made for spatially uniform illumination conditions, i.e., no dependence on $m, n$. Full scene data vectors are defined as,

$$
\begin{aligned}
& \mathbf{L}_{w}^{(t)}=\left[\left(\mathbf{L}^{(t)}[1,1]\right)^{T}, \ldots,\left(\mathbf{L}^{(t)}[M, N]\right)^{T}\right]^{T}, t=1,2 \\
& \mathbf{L}=\left[\begin{array}{l}
\mathbf{L}_{w}^{(1)} \\
\mathbf{L}_{w}^{(2)}
\end{array}\right]
\end{aligned}
$$

where the size $2 M N K$ vector $\mathbf{L}$ contains all the data measurements. Consequently, the cost function associated with the basis coefficients with $\mathbf{x}_{\epsilon}=\left[\left(\boldsymbol{\epsilon}^{(1)}\right)^{T},\left(\boldsymbol{\epsilon}^{(2)}\right)^{T}\right]^{T}$ is given by,

$$
f\left[\mathbf{x}_{\epsilon}\right]=\mathbf{L}^{T} \mathbf{L}+\mathbf{x}_{\epsilon}^{T} \mathbf{A}_{\epsilon}^{T} \mathbf{A}_{\epsilon} \mathbf{x}-2 \mathbf{L}^{T} \mathbf{A}_{\epsilon} \mathbf{x}
$$

where

$$
\mathbf{A}_{\epsilon}=\left[\begin{array}{cc}
\mathbf{A}_{\epsilon}^{(1)} & \mathbf{0} \\
\mathbf{0} & \mathbf{A}_{\epsilon}^{(2)}
\end{array}\right]
$$


and

$$
\mathbf{A}_{\epsilon}^{(t)}=\left[\begin{array}{c}
\mathbf{A}_{\epsilon}^{(t)}[1,1] \\
\vdots \\
\mathbf{A}_{\epsilon}^{(t)}[M, N]
\end{array}\right] t=1,2
$$

Simplifying and removing terms not dependent on $\mathbf{x}_{\epsilon}$ produces a cost function given by,

$$
f\left[\mathbf{x}_{\epsilon}\right]=\frac{1}{2} \mathbf{x}_{\epsilon}^{T} \mathbf{G}_{\epsilon} \mathbf{x}_{\epsilon}+\mathbf{c}^{T} \mathbf{x}_{\epsilon}
$$

where

$$
\begin{aligned}
& \mathbf{G}_{\epsilon}=2 \mathbf{A}_{\epsilon}^{T} \mathbf{A}_{\epsilon} \\
& \mathbf{c}_{\epsilon}=-2 \mathbf{A}_{\epsilon}^{T} \mathbf{L}
\end{aligned}
$$

Proving $\mathbf{G}_{\epsilon}$ is positive semi-definite is not straightforward. Initial numerical testing on simulated data sets does show positive semi-definiteness.

\subsubsection{Initialization}

Initialization of the model parameter vector $\mathbf{x}$ remains an integral part of the iterative optimization process due to the complexity of the problem and the alternating optimization approach utilized. Poor initialization may result in convergence to a local minimum resulting in poor change detection performance. Estimation of the reflectance vectors is chosen as the first step in the AO method; therefore, initial estimates of only the shadow coefficients $\mathbf{x}_{s}$ and RT basis coefficients $\mathbf{x}_{\epsilon}$ are required. Current methods exist in the literature for estimating atmospheric illumination parameters within hyperspectral images and can typically be found within the suite of atmospheric compensation algorithms used for HSI. Commonly used methods include an empirical line method (ELM) based on the normalized difference vegetation index (NDVI) [43], the QUick Atmospheric Correction (QUAC) method [8] and the Fast Line-of-sight Atmospheric Analysis of Spectral Hypercubes (FLAASH) algorithm [63]. 
The NDVI and QUAC methods essentially provide an estimate of the path radiance term $\mathbf{L}_{p}$ and the combined transmission-illumination term $\boldsymbol{\tau} \odot \mathbf{L}_{s}+\boldsymbol{\tau} \odot \mathbf{L}_{d}$ via automated methods using in-scene information and library reflectance signatures. Since the RT basis coefficients are coupled, the path radiance term can be used to estimate the RT basis coefficients. The FLAASH algorithm utilizes a forward-modeling approach using MODTRAN along with known and estimated atmospheric/collection parameters to obtain the RT terms associated with the data model. These atmospheric compensation algorithms can potentially be used to obtain an initial estimate for the basis coefficients via projection using the pre-computed RT subspace basis vectors. As a simple alternative to these algorithms, one can use prior information about the conditions of the data collection (sensor altitude, time of day, viewing geometry, etc.) as inputs to MODTRAN to directly compute an initial estimate of the RT parameters and corresponding basis coefficients. This latter approach may not be as accurate as using FLAASH, which estimates important atmospheric parameters such as water column and visibility, but nonetheless provides a simple alternative for obtaining initial estimates.

Initialization of the shadow coefficients is challenging as well as little prior information is available. One simple approach is to identify shadow pixels based upon histogram thresholding. One can determine the Euclidean norm of each spectral vector in the scene and classify a certain percentage of the smallest norm vectors as shadows. These pixels would be initialized with shadow coefficients of $\alpha=d_{1}$ and $\beta=d_{2}$ where $d_{1}$ would be a value close to or equal to zero and $d_{2}$ would be small but slightly larger than $d_{1}$, because a pixel in shadow can still receive a fair amount 
of indirect illumination. Consequently, one would expect $\beta>\alpha$ for shadow pixels. The remaining pixels would be considered non-shadow and would be initialized with $\alpha=\beta=1$. Additionally, an initial estimate for the surface geometry parameter $\theta$ is required, since this term is combined with the shadow coefficient for $\tilde{\alpha}$. One can potentially use either $\theta=0$ for cases of complex scene surface geometry, or $\theta=\theta_{s}=$ Solar Zenith Angle. These initialization methods for the shadow coefficients are applied separately for the time- 1 and time- 2 data sets.

\subsubsection{Convergence Criteria}

Convergence criteria are required in an optimization routine to determine when the minimum of the cost function has been reached and the algorithm can stop iterating. Typical optimization algorithms utilize first and second order optimality conditions to determine convergence. Specifically, for unconstrained optimization, a local minimum exists if the gradient of the cost function is zero and the Hessian matrix is positive definite. For constrained optimization, the optimality conditions are given by the Karush-Kuhn-Tucker (KKT) conditions, which utilize the Lagrangian rather than simply the gradient and Hessian of the cost function [32]. These optimality criteria are difficult to compute for the optimization problem at hand. Consequently, other convergence criteria are examined. Intuitively, the AO algorithm should stop when the state estimates do not change very much from one iteration to the next. However, the amount of change considered significant is difficult to define. One straight-forward metric measuring the change in estimates is simply the norm of the difference vector of the state estimates at iteration $k$ and $k+1$. The difference vector is defined as,

$$
\Delta \hat{\mathbf{x}}^{(k+1)}=\hat{\mathbf{x}}^{(k+1)}-\hat{\mathbf{x}}^{(k)}
$$


where $\hat{\mathbf{x}}^{(k)}$ is the estimate of the state vector at iteration $k$. One can then define the magnitude of the difference vector as a convergence metric,

$$
\Psi^{(k+1)}=\left\|\Delta \hat{\mathbf{x}}^{(k+1)}\right\|
$$

One potential problem with this metric is that it weighs changes in all elements of the state vector equally. The leading RT basis coefficient $\epsilon_{1}^{(t)}$ can take values on the order of $10^{2}$ to $10^{4}$ whereas all the shadow coefficient and reflectance vector estimates will take values between 0 and 1 . A change of around 0.25 in the reflectance estimate from one iteration to the next is significant. However, a similar change in the leading basis coefficient estimate is not as significant.

With these considerations in mind, the elements of the difference vector given in (2.55) can be weighted by the elements of the state vector $\hat{\mathbf{x}}^{(k+1)}$ before taking the norm of the vector. This results in a convergence metric given by,

$$
\tilde{\Psi}^{(k+1)}=\left\|\frac{\Delta \hat{\mathbf{x}}^{(k+1)}}{\hat{\mathbf{x}}^{(k+1)}}\right\|
$$

where an element-by-element vector division is utilized. This weighting approach encounters problems when there exist state estimates that are zero or nearly zero. Since the shadow coefficients regularly take values of zero or nearly zero for shadow pixels, this issue must be addressed. Consequently, a small positive constant can be added to prevent this from occurring, which results in,

$$
\tilde{\Psi}^{(k+1)}=\left\|\frac{\Delta \hat{\mathbf{x}}^{(k+1)}}{\hat{\mathbf{x}}^{(k+1)}+\delta}\right\|
$$

where $\delta$ is a small positive constant. Regardless of which convergence metric is used, the problem still remains determining a threshold for the stopping point using that convergence criterion. This threshold should be dependent on the size of the data 
set and subsequent parameter vector. Perhaps a linear relationship can be used to determine the threshold as

$$
\eta(M N)=a M N+b
$$

where $\eta$ is the threshold and $M N$ is the number of pixels in the scene. One can also consider convergence criteria related to the change in residual optimization error from one iteration to the next. Further work in developing appropriate convergence criteria and stopping points is required. For the results provided in Chapter 4, the stopping criteria was based upon visual inspection of state estimates and corresponding change detection performance after varying the number of iterations.

\subsubsection{Performance Metrics}

In order to compare and assess the viability and the performance of the proposed MB method for various cases with those discussed in the literature, a measure of performance must be used. A common performance metric used in probabilistic detection theory is a receiver operating characteristic (ROC) curve [45]. The ROC curve plots the probability of detection $P_{d}$ as a function of the probability of false alarm $P_{f}$. In general, it is difficult to discuss optimality when using a GLRT because of the need to estimate unknown parameters. Additionally, the theoretical performance of the change detection algorithm depends upon both the change in reflectance and the change in RT terms as discussed earlier. However, an empirical ROC curve plot can still be utilized to assess the relative performance of algorithms for a particular data set.

The distribution of the detection statistic in relation to the theoretical distribution can also be analyzed to assess estimation performance and/or the estimated noise 
model applied for the data. The detection statistic given in (2.16) is of the form,

$$
\tilde{D}_{G}[m, n \mid \mathbf{x}]=D^{(1)}[m, n \mid \mathbf{x}]+D^{(2)}[m, n \mid \mathbf{x}]
$$

Due to the independent Gaussian noise assumptions associated with the data, $D^{(1)}$ and $D^{(2)}$ are expected to be distributed according to a $\chi^{2}$ distribution with $K$ degrees of freedom. From the temporal independence assumption, $D^{(1)}$ and $D^{(2)}$ are also independent. The sum of independent $\chi^{2}$ random variables is also a $\chi^{2}$ random variable with degrees of freedom equal to the sum of the individual degrees of freedom. Consequently, the detection statistic will have an expected distribution

$$
\tilde{D}_{G} \sim \chi^{2}(2 K)
$$

with mean and variance given by

$$
\begin{aligned}
& E\left\{\tilde{D}_{G}\right\}=2 K \\
& \operatorname{var}\left\{\tilde{D}_{G}\right\}=4 K
\end{aligned}
$$

The actual sample distribution associated with the data can provide a measure of estimation error and model mismatch. The estimate bias error is defined as

$$
b^{(t)}[m, n]=\left\|\boldsymbol{\mu}_{0}^{(t)}[m, n]-\hat{\boldsymbol{\mu}}_{0}^{(t)}[m, n \mid \hat{\mathbf{x}}]\right\|^{2}
$$

for $t=1,2$. As the bias error increases, the distribution of $D_{G}$ will no longer take the form of a $\chi^{2}$. The bias error is estimated here because of the subspace approximation employed in the data model. As the subspace dimension $I$ decreases, the subspace approximation error will increase, resulting in a larger bias error. As a result, one might believe that a larger $I$ is desirable. However, a trade off exists between the bias error, the estimation error, and detection performance. As $I$ increases, the degrees of freedom for the RT terms of the model increase, making the estimation problem 
more difficult. Additionally, the increased degrees of freedom due to a larger $I$ can potentially suppress error due to actual subtle changes in reflectance as the change in signal level can be more readily explained by the model by adjusting the increased number of basis coefficients associated with the less significant eigenvectors. Further consideration of optimal rank reduction for detection problems may be found in [70]. Consequently, one would like to choose an appropriate subspace dimensionality $I$, such that the bias error is relatively small while at the same time making the dimensionality of the estimation problem small. Initial experimenting (Section 4.2.3) has shown that detection performance generally peaks for some value of $I$ greater than 1 but much less than $K$, typically between $10-20$ when $K$ is typically greater than 100 . For the work performed here, $I=10$ is used.

An additional metric that can provide insight for detection performance, especially in cases with few target pixels, is a simple signal to clutter ratio (SCR). Here a simple empirical SCR is proposed to assess separation for each change target and is defined by,

$$
S C R[m, n \mid \hat{\mathbf{x}}]=\frac{\tilde{D}_{G}[m, n \mid \hat{\mathbf{x}}]-\hat{\mu}_{b}}{\hat{\sigma}_{b}}, m, n \in \mathcal{P}_{c}
$$

where $\mathcal{P}_{c}$ are the pixel indices associated with change targets with cardinality $\left|\mathcal{P}_{c}\right|=$ $P_{c}$ and

$$
\hat{\mu}_{b}=\frac{1}{M N-P_{c}} \sum_{m, n \notin \mathcal{P}_{c}} \tilde{D}_{G}[m, n \mid \hat{\mathbf{x}}]
$$

and

$$
\hat{\sigma}_{b}^{2}=\frac{1}{M N-P_{c}-1} \sum_{m, n \notin \mathcal{P}_{c}}\left(\tilde{D}_{G}[m, n \mid \hat{\mathbf{x}}]-\hat{\mu}_{b}\right)^{2}
$$


are the sample mean and sample variance of the background (no-change) pixels respectively. One can assess the average SCR as well, given by,

$$
\hat{\mu}_{S C R}=\frac{\hat{\mu}_{t}-\hat{\mu}_{b}}{\hat{\sigma}_{b}}
$$

where

$$
\hat{\mu}_{t}=\frac{1}{P_{c}} \sum_{m, n \in \mathcal{P}_{c}} \tilde{D}_{G}[m, n \mid \hat{\mathbf{x}}] .
$$

These performance metrics are used as a tool for assessing and comparing performance of the MB method along with other algorithms currently used. In general, one expects strong correlation between SCR and ROC performance, i.e., a larger SCR would indicate better ROC performance due to improved separation between target and background detector outputs.

\subsection{Spectral Smoothing of Reflectance}

In the optimization routine, only bound constraints are placed on the estimates of the reflectance vector $\boldsymbol{\rho}$. However, from experience in working with hyperspectral data and observation of a number of material reflectance spectra, one observes that the majority of solid materials have reflectance that varies slowly as a function of wavelength. Figure 2.4 displays the reflectance spectra of a number of materials that exhibit this spectrally smooth characteristic. Consequently, a constraint can be imposed that forces the reflectance estimates to be smooth functions of wavelength to improve not only the estimates of the reflectance vectors but potentially the resulting estimates of the RT terms as well by not allowing fine features typically due to atmospheric variations to be modeled in the reflectance component of the model. Two avenues are explored to achieve this smoothing: 1) low-pass filtering the reflectance 


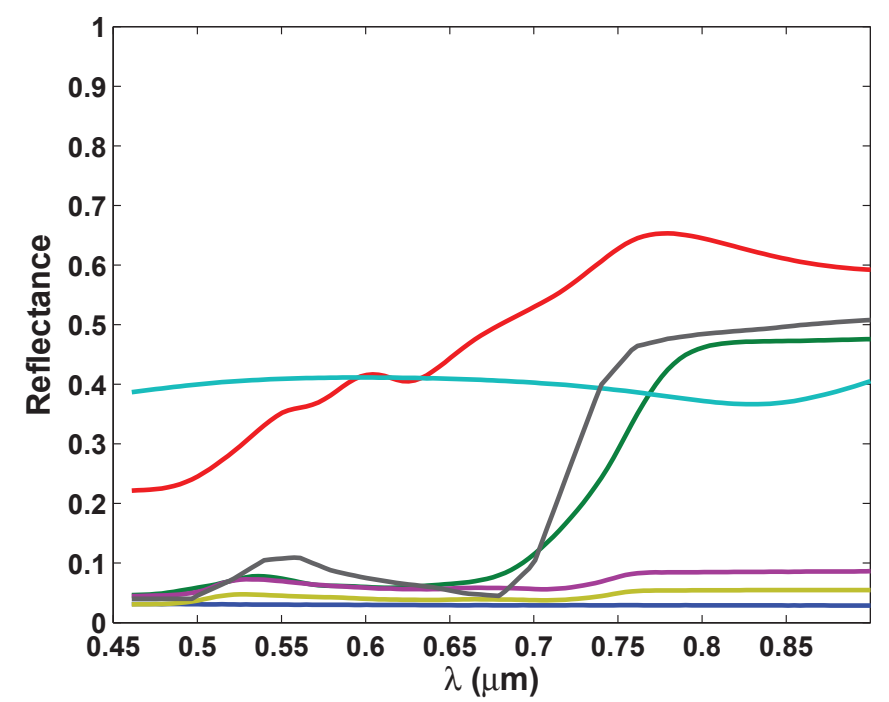

Figure 2.4: Reflectance spectral for a number of materials, which exhibit spectrally smooth characteristics

estimates, and 2) using a subspace approximation for the reflectance vectors where the basis functions are smooth.

\subsubsection{Smoothing via Filtering}

In the first method, a low-pass filter is applied to the reflectance estimates directly after the reflectance estimation step of the AO routine. The filtering is described by

$$
\rho_{f}\left[\lambda_{k}\right]=\sum_{n_{f}=-\left(N_{f}-1\right) / 2}^{\left(N_{f}-1\right) / 2} \rho\left[\lambda_{k}+n_{f}\right] h\left[n_{f}\right]
$$

where $h$ is an (odd) length- $N_{f}$, symmetric, finite impulse response (FIR), low-pass filter. The shape and size of the filter is developed to achieve adequate smoothing without removing important spectral features of the material. One can equivalently filter the data measurements $L$ for the reflectance estimation step to achieve the same results as smoothing the reflectance vectors themselves. In general, the shape 
and size of the filter will depend on the spectral resolution of the sensor used for data collection. A sensor with much finer spectral resolution resulting in more atmospheric features will require a longer smoothing filter. The sensors used in this work generally have lower spectral resolution with relatively smooth measurements to begin with. As a result, simple, small length filters are used for the smoothing step. Future work will examine possible methods for optimizing the smoothing filter and the impact of optimizing the filter on detection/estimation performance.

\subsubsection{Smoothing via Subspace Constraint}

A second approach to achieve smooth reflectance estimates is to represent the reflectance using a subspace approximation where the bases chosen for the subspace are smooth functions of wavelength. By choosing an appropriate set of basis vectors, one can essentially enforce the smoothness constraint, while at the same time simplifying the estimation problem by reducing the number of variables to estimate. Under the subspace approximation, the reflectance is given by

$$
\boldsymbol{\rho}[m, n] \approx \mathbf{R} \gamma[m, n]
$$

where $\mathbf{R}$ is the matrix whose columns contain the reflectance basis vectors determined from the chosen basis function and $\gamma$ is the length $I_{r}$ vector containing the respective basis coefficients for pixel $(m, n)$. Using this approximation, the change detection model is now given by,

$$
\begin{aligned}
& \mathbf{L}^{(1)}[m, n] \approx \mathbf{R} \boldsymbol{\gamma}^{(1)}[m, n] \odot\left(\tilde{\alpha}^{(1)}[m, n] \mathbf{S}^{(1)} \boldsymbol{\epsilon}^{(1)}\right. \\
& \left.\quad+\beta^{(1)}[m, n] \mathbf{D}^{(1)} \boldsymbol{\epsilon}^{(1)}\right)+\mathbf{P}^{(1)} \boldsymbol{\epsilon}^{(1)}+\boldsymbol{\nu}^{(1)}[m, n] \\
& \mathbf{L}^{(2)}[m, n] \approx \mathbf{R} \boldsymbol{\gamma}^{(2)}[m, n] \odot\left(\tilde{\alpha}^{(2)}[m, n] \mathbf{S}^{(2)} \boldsymbol{\epsilon}^{(2)}+\beta^{(2)}[m, n] \mathbf{D}^{(2)} \boldsymbol{\epsilon}^{(2)}\right) \\
& \quad+\mathbf{P}^{(2)} \boldsymbol{\epsilon}^{(2)}+\boldsymbol{\nu}^{(2)}[m, n]
\end{aligned}
$$


Under this formulation, the change detection hypothesis test is given by,

$$
\begin{array}{ll}
H_{0}: & \gamma^{(1)}[m, n]=\gamma^{(2)}[m, n] \\
H_{1}: & \gamma^{(1)}[m, n] \neq \gamma^{(2)}[m, n]
\end{array}
$$

The key for this approach is choosing an appropriate basis that will enforce the smoothness constraint. Here the use of the Legendre polynomials is examined as the basis functions [24]. Legendre functions $\mathbf{r}_{n}$ are solutions to Legendre's differential equation, given by,

$$
\frac{d}{d \lambda}\left[\left(1-\lambda^{2}\right) \frac{d}{d \lambda} \mathbf{r}_{n}(\lambda)\right]+n(n+1) \mathbf{r}_{n}(\lambda)=0
$$

The solutions to this differential equation can be defined by,

$$
\mathbf{r}_{n}(\lambda)=\sum_{p=0}^{n}(-1)^{k}\left(\begin{array}{l}
n \\
p
\end{array}\right)\left(\frac{1+\lambda}{2}\right)^{n-p}\left(\frac{1-\lambda}{2}\right)^{p}
$$

The Legendre polynomials have the important property of being orthogonal with respect to the $\mathcal{L}^{2}$ inner product defined on the interval $-1 \leq \lambda \leq 1$,

$$
\int_{-1}^{1} \mathbf{r}_{n}(\lambda) \mathbf{r}_{j}(\lambda) d \lambda=\frac{2}{2 n+1} \delta_{n j}
$$

where $\delta_{n j}$ represents the Kronecker delta function. In application, these functions must be shifted and scaled appropriately to apply to the wavelength range of the hyperspectral data. Figure 2.5 displays the first few Legendre Polynomials shifted appropriately for application to a spectral range of $0.4-2.5 \mu \mathrm{m}$. The basis approximation is applied to a vegetation reflectance vector and a camouflage reflectance vector using $I_{r}=20$ and $I_{r}=40$ in Figure 2.6. The approximation error is relatively small using a subspace dimension of $I_{r}=20$ and nearly zero for $I_{r}=40$. The original number of spectral bands is $K=100$, which results in a $60 \%$ decrease in the number of parameters to estimate while forcing smoother reflectance estimates. The challenge 


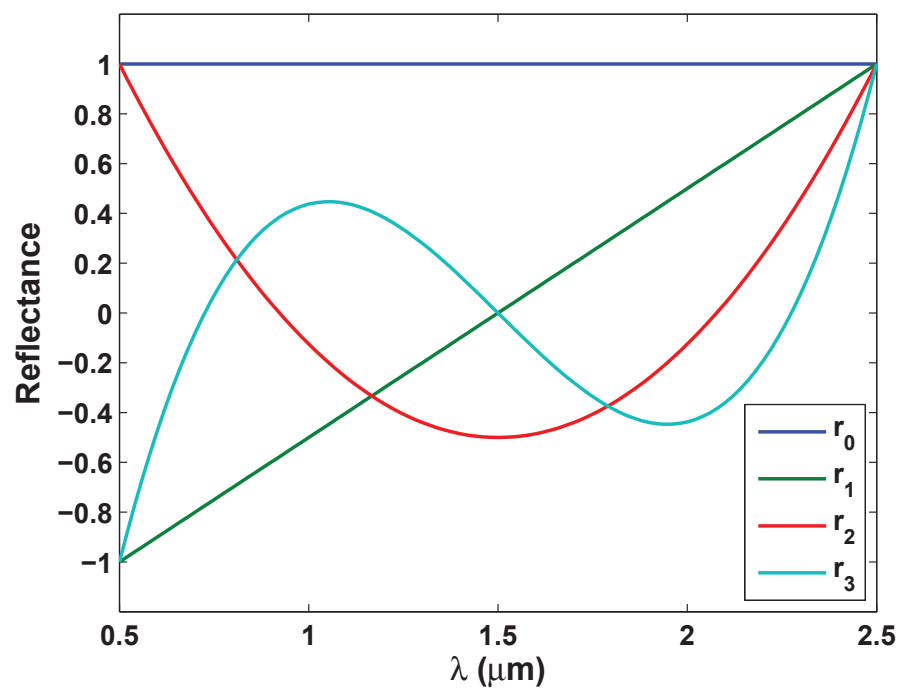

Figure 2.5: Legendre Polynomials

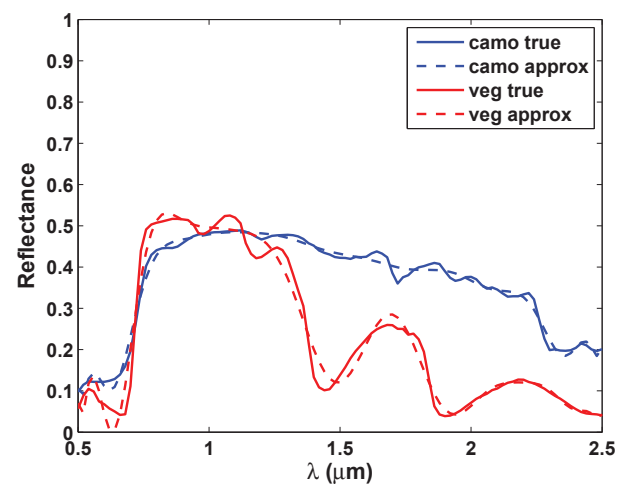

(a) $I_{r}=20$

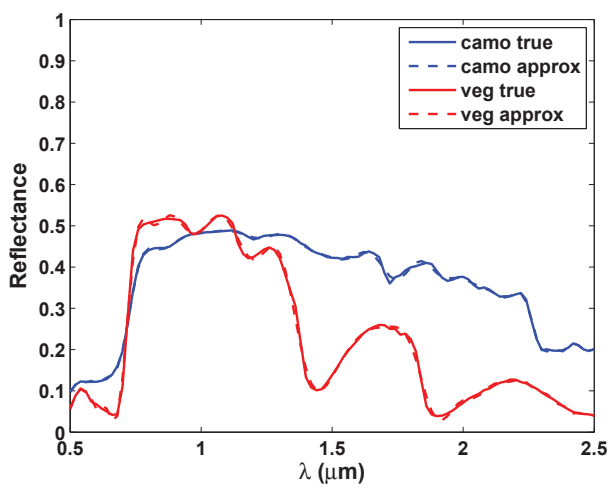

(b) $I_{r}=40$

Figure 2.6: Examination of subspace approximation for vegetation and camouflage reflectance using Legendre polynomials with varying subspace dimensionality 
is to choose a large enough $I_{r}$ such that the approximation error will be small without allowing for non-smooth estimates. Vegetation represents one of the less smooth reflectance functions in various databases examined. Consequently, $I_{r}=40$ is chosen for use in this work.

\subsection{Extension to Uncalibrated and Relatively Calibrated Data}

One current limitation of the model presented to this point is the requirement for data calibrated to physical units of spectral radiance. The model given in (2.2) represents sensor-reaching radiance. The output of the digital camera is a function of this sensor reaching radiance. An absolute calibration procedure is applied to the uncalibrated imagery collected by the camera in order to relate the digital numbers produced to physical radiance units. Here, the possibility of applying the modelbased change detection algorithm to raw, uncalibrated imagery or data that has been relatively calibrated to remove sensor non-uniformity is discussed. Application to uncalibrated or relatively calibrated data may provide an advantage over processing on absolutely calibrated imagery, especially for cases in which residual calibration error exists due to poor calibration data.

In order to explicitly define the change detection problem associated with uncalibrated imagery or relatively calibrated imagery, brief discussion is given to hyperspectral data collection and calibration procedures. The hyperspectral data cube contains two spatial dimensions indexed with $m$ and $n$ and one spectral dimension indexed with $k$ as previously discussed. Pushbroom hyperspectral imaging systems collect one spatial dimension $(m)$ and the spectral dimension $(k)$ concurrently, and build the third dimension $(n)$ by collecting multiple frames as the imaging platform 
moves as demonstrated in Figure 2.7(a). This can be thought of as collecting a spatial
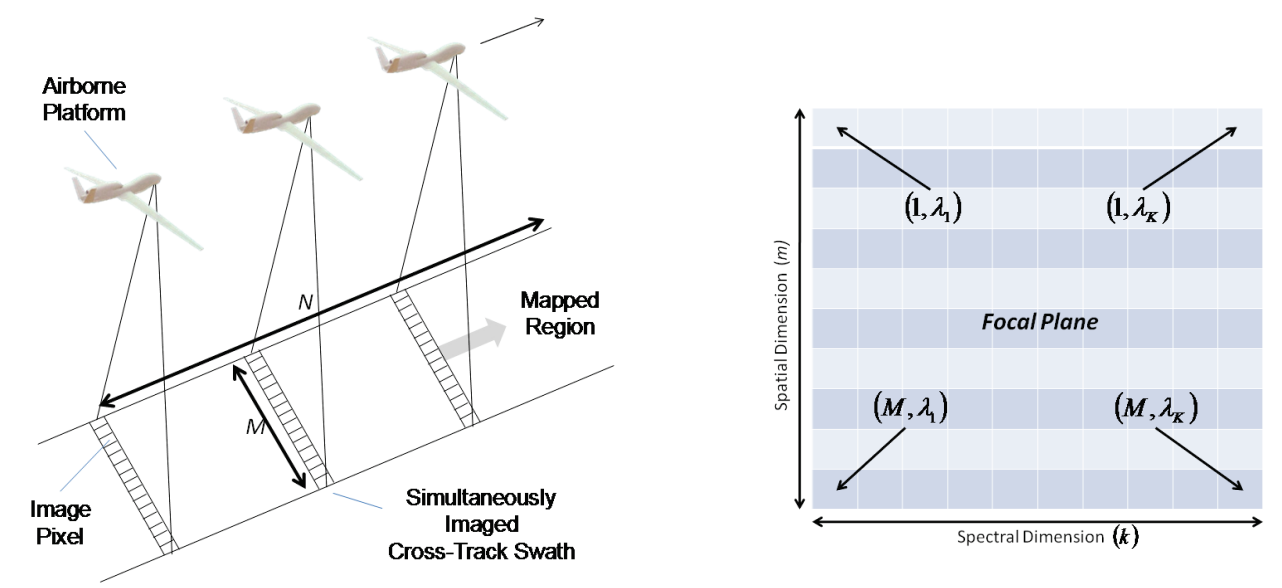

\section{(a) Pushbroom imaging system (b) Spatial and spectral dispersion of light collected at each frame}

Figure 2.7: General operation of pushbroom imaging spectrometer. The system collects spectral and spatial information concurrently while the second spatial dimension is collected over time by collecting multiple frames as the imaging platform moves.

slice of the scene at $K$ different wavelengths with each frame captured by the imaging system. The spectral information for a spatial column of the scene is dispersed across the columns of the focal plane detector array as shown in Figure 2.7(b). As a result of this sensing modality, the same detector element on the focal plane is used to image an entire spatial row at a particular wavelength, which is important to understand when analyzing both the calibration procedure and the noise characteristics of the data cube.

A linear model describing the relationship between the deterministic pupil-plane spectral radiance $L[m, n, k]$ described in (2.2), (or the subspace approximation given 
in (2.4)) to the mean of the detected photo-electrons $N_{p}[m, n, k]$ is given by,

$$
\begin{aligned}
\mu_{N_{p}}[m, n, k] & =E\left\{N_{p}[m, n, k]\right\} \\
& =\left(\frac{\pi}{4(f / \#)^{2}+1} K_{\mathrm{opt}}[m, k] \eta\left[\lambda_{k}\right] \tau_{\mathrm{opt}}\left[\lambda_{k}\right] \frac{\lambda_{k}}{h c} t_{d} \Delta \lambda_{k} A_{d}\right) L[m, n, k],
\end{aligned}
$$

where

$$
\begin{aligned}
& f / \#=\text { Optics effective f-number } \\
& K_{\text {opt }}=\text { Optics cosine roll-off function } \\
& \eta=\text { Detector quantum efficiency } \\
& \tau_{\text {opt }}=\text { Effective optics transmission } \\
& \lambda_{k}=\text { Center wavelength for band } k \\
& t_{d}=\text { Frame integration time } \\
& \Delta \lambda_{k}=\text { Spectral bandwidth for band } k \\
& A_{d}=\text { Detector area }
\end{aligned}
$$

and where $E\{\}$ denotes the temporal expected value. The uncalibrated sensor output in data numbers $(\mathrm{DN})$ due to the signal component can be related to the integrated photoelectrons per frame by,

$$
r[m, n, k]=g[m, k] N_{p}[m, n, k]
$$

where $g[m, k]$ is a photoelectron conversion factor (units of $\mathrm{DN} /$ photoelectron). The dependence of $g$ on $m$ and $k$ is indicative of pixel response non-uniformity (PRNU), which is typically corrected during post-processing calibration of the data. An effective sensor gain coefficient can be defined as,

$$
a[m, k]=g[m, k]\left[\frac{\pi}{4(f / \#)^{2}+1} K_{\mathrm{opt}}[m, k] \eta\left[\lambda_{k}\right] \tau_{\mathrm{opt}}\left[\lambda_{k}\right] \frac{\lambda_{k}}{h c} t_{d} \Delta \lambda_{k} A_{d}\right] .
$$


Assuming a dark current offset $b[m, k]$, the uncalibrated hyperspectral data are related to the incoming radiance by the linear model,

$$
r[m, n, k]=a[m, k] L[m, n, k]+b[m, k]+\nu_{s}[m, n, k]+\nu_{i}[m, n, k]
$$

where $\nu_{s}[m, n, k]$ is a zero-mean, signal-dependent noise with variance dependent on $L[m, n, k]$, and $\nu_{i}[m, n, k]$ is a zero-mean, signal-independent noise resulting from dark current shot noise, read noise (includes amplifier, reset, and other electronic noise sources), and quantization noise [40]. The noise terms are assumed to be independent of each other as both originate from different physical processes. Techniques for estimating the statistics of the noise components of the model are presented in Chapter 3 .

Typically, post-processing routines are applied to uncalibrated hyperspectral data as collected by the sensor to remove sensor artifacts and to improve the SNR of the data. Sensor artifacts include the PRNU and dark current offset discussed earlier as well as smile and keystone spectral distortion [76]. Post-processing algorithms focused primarily on removing PRNU and dark current offset are often referred to as nonuniformity correction (NUC) or radiometric calibration. The SNR can be improved by spatially or spectrally filtering the data, which reduces noise at the expense of spatial and/or spectral resolution respectively.

The dark current offset given by $b[m, k]$ in $(2.77)$ can be corrected by subtracting the estimated mean dark current level for each pixel, an estimate of which can be obtained simply by averaging a number of output frames with no illumination present $(L=0)$. PRNU is corrected using a spatially uniform light source for which the relative magnitude of the output illumination is known. Note that if the exact output illumination is known for the source, a direct estimate of $a[m, k]$ in $(2.77)$ can 
be obtained, which is typically referred to as an "absolute" radiometric calibration. An absolute calibration converts the uncalibrated data directly to sensor-reaching radiance $\mathbf{L}[m, n]$. Otherwise, a relative calibration procedure is applied for which an unknown gain and offset exists between the relatively calibrated data and the sensor-reaching radiance described by $\mathbf{L}[m, n]$.

The NUC corrected data is given by,

$$
c[m, n, k]=\frac{r[m, n, k]-b_{c}[m, k]}{a_{c}[m, k]},
$$

which is described similarly by Schott [76]. Note the same NUC is applied for all $N$ frames in the uncalibrated data cube since the same detector element is responsible for those measurements, as is evident in Figure 2.7. If an absolute calibration is being performed, $b_{c}[m, k]=\hat{b}[m, k], a_{c}[m, k]=\hat{a}[m, k]$, and $c[m, n, k]=\hat{L}[m, n, k]$. Otherwise, the calibration gain and offset are left general and the calibrated data $c$ has simply been corrected for nonuniformity in the uncalibrated data.

For extension to the change detection problem, the uncalibrated data at time $t$, $\mathbf{r}^{(t)}$, is related to the sensor reaching radiance of the original physical model in (2.4) by,

$$
\mathbf{r}^{(t)}[m, n]=\mathbf{a}^{(t)}[m] \odot \mathbf{L}^{(t)}[m, n]+\mathbf{b}^{(t)}[m]+\boldsymbol{\nu}_{s}[m, n]+\boldsymbol{\nu}_{i}[m, n]
$$

where vector notation is utilized. The gain $\mathbf{a}$ and offset $\mathbf{b}$ are dependent on only one spatial index $(m)$ for pushbroom/whiskbroom imaging systems. Depending on the stability of the imaging system, one may be able to ignore the temporal dependence of the effective sensor gain, i.e., $\mathbf{a}^{(t)}[m]=\mathbf{a}[m]$. However, the temporal dependence is retained at this time to allow for more general application. Substitution using (2.4) 
yields,

$$
\begin{gathered}
\mathbf{r}^{(t)}[m, n]=\mathbf{a}^{(t)}[m] \odot \boldsymbol{\rho}^{(t)}[m, n] \odot\left(\tilde{\alpha}^{(t)}[m, n] \mathbf{S}^{(t)} \boldsymbol{\epsilon}^{(t)}+\beta^{(t)}[m, n] \mathbf{D}^{(t)} \boldsymbol{\epsilon}^{(t)}\right) \\
+\mathbf{a}^{(t)}[m] \odot \mathbf{P}^{(t)} \boldsymbol{\epsilon}^{(t)}+\mathbf{b}^{(t)}[m]
\end{gathered}
$$

The number of measurements for a single data cube is $M N K$, and the number of unknown model parameters equal to $M N K+2 M K+2 M N+I$. If a reflectance subspace is used for the reflectance vectors with subspace dimensionality given by $I_{r}$, the number of unknown parameters reduces to $M N I_{r}+2 M K+2 M N+I$, which may be less than the number of measurements. Assuming a reflectance subspace is not utilized, the estimation problem is ill-posed for a single data cube at time $t$. For the change detection problem, two data cubes $t=1,2$ exist with a total of $2 M N K$ measurements. Under the null hypothesis of the change detection problem, the total number of unknown parameters for the joint data model is $K M N+4 M K+4 M N+2 I$, which typically will be less than the number of measurements. For example, let us assume $M=200, N=200, K=100$, and $I=10$. The total number of unknown parameters is $4,000,000+80,000+160,000+20=4,240,020$ with a total of $8,000,000$ measurements, resulting in an overdetermined system.

The calibrated data is given by,

$$
\mathbf{c}^{(t)}[m, n]=\tilde{\mathbf{a}}_{c}^{(t)}[m] \odot\left(\mathbf{r}^{(t)}[m, n]-\mathbf{b}_{c}^{(t)}[m]\right)
$$

where

$$
\tilde{\mathbf{a}}_{c}^{(t)}[m]=\left[1 / a_{c}^{(t)}[m, 1], \ldots, 1 / a_{c}^{(t)}[m, K]\right]^{T}
$$

Using this relative linear correction, the calibrated data model is given by,

$$
\begin{aligned}
& \mathbf{c}^{(t)}[m, n]=\tilde{\mathbf{a}}_{c}^{(t)}[m] \odot \mathbf{a}^{(t)}[m] \odot \boldsymbol{\rho}^{(t)}[m, n] \odot\left(\tilde{\alpha}^{(t)}[m, n] \mathbf{S}^{(t)} \boldsymbol{\epsilon}^{(t)}\right. \\
& \left.\quad+\beta^{(t)}[m, n] \mathbf{D}^{(t)} \boldsymbol{\epsilon}^{(t)}\right)+\tilde{\mathbf{a}}_{c}^{(t)}[m] \odot \mathbf{a}^{(t)}[m] \odot \mathbf{P}^{(t)} \boldsymbol{\epsilon}^{(t)} \\
& \quad+\tilde{\mathbf{a}}_{c}^{(t)}[m] \odot\left(\mathbf{b}^{(t)}[m]-\mathbf{b}_{c}^{(t)}[m]\right)
\end{aligned}
$$


The noise terms are omitted at this time as more discussion is given to these terms in the following chapter describing noise estimation. This model can be simplified using a general gain and offset as,

$$
\begin{aligned}
\mathbf{c}^{(t)}[m, n] & =\mathbf{a}_{g}^{(t)}[m] \odot \boldsymbol{\rho}^{(t)}[m, n] \odot\left(\tilde{\alpha}^{(t)}[m, n] \mathbf{S}^{(t)} \boldsymbol{\epsilon}^{(t)}+\beta^{(t)}[m, n] \mathbf{D}^{(t)} \boldsymbol{\epsilon}^{(t)}\right) \\
& +\mathbf{a}_{g}^{(t)}[m] \odot \mathbf{P}^{(t)} \boldsymbol{\epsilon}^{(t)}+\mathbf{b}_{g}^{(t)}[m]
\end{aligned}
$$

where

$$
\begin{aligned}
\mathbf{a}_{g}^{(t)}[m] & =\tilde{\mathbf{a}}_{c}^{(t)}[m] \odot \mathbf{a}^{(t)}[m] \\
\mathbf{b}_{g}^{(t)}[m] & =\tilde{\mathbf{a}}_{c}^{(t)}[m] \odot\left(\mathbf{b}^{(t)}[m]-\mathbf{b}_{c}^{(t)}[m]\right)
\end{aligned}
$$

Typically, the relative offset $\mathbf{b}_{c}^{(t)}$ is an estimate of the dark current offset, i.e.,

$$
\mathbf{b}_{c}^{(t)}[m]=\hat{\mathbf{b}}^{(t)}[m]
$$

in which case $\mathbf{b}^{(t)}[m]-\mathbf{b}_{c}^{(t)}[m] \approx 0$ assuming a good estimate exists. In this case, $\mathbf{b}_{g}^{(t)}[m] \approx 0$ and the estimation problem simplifies to one of estimating $\mathbf{a}_{g}^{(t)}[m]$ alone. However, retention of $\mathbf{b}_{g}^{(t)}[m]$ allows for compensation of residual calibration error. Additionally, for an absolute radiometric calibration, $\mathbf{a}_{c}^{(t)}[m]=\hat{\mathbf{a}}(t)[m]$. Consequently, $\mathbf{a}_{g}(t)[m] \approx 1$, and the data model simplifies to the original sensor-reaching radiance model of $\mathbf{L}^{(t)}$. The number of unknowns for the relatively calibrated data model remains the same as the uncalibrated data model.

The question remains on how to solve the estimation problem associated with uncalibrated or relatively calibrated data. With the reflectance, RT basis coefficients, and shadow coefficients known, the model is linear with respect to the sensor gain and offset. Consequently, a fourth step in the alternating optimization process could be used to estimate the respective gain and offset terms associated with the model. The new unknown parameter vector is given by,

$$
\mathbf{x}=\left[\mathbf{x}_{\rho}^{T}, \mathbf{x}_{s}^{T}, \mathbf{x}_{\epsilon}^{T}, \mathbf{x}_{c}^{T}\right]^{T}
$$


where

$$
\begin{gathered}
\mathbf{x}_{c}=\left[a^{(1)}[1], \ldots, a^{(1)}[M], b^{(1)}[1], \ldots, b^{(1)}[M],\right. \\
\left.a^{(2)}[1], \ldots, a^{(2)}[M], b^{(2)}[1], \ldots, b^{(2)}[M]\right]
\end{gathered}
$$

or

$$
\begin{gathered}
\mathbf{x}_{c}=\left[a_{g}^{(1)}[1], \ldots, a_{g}^{(1)}[M], b_{g}^{(1)}[1], \ldots, b_{g}^{(1)}[M],\right. \\
\left.a_{g}^{(2)}[1], \ldots, a_{g}^{(2)}[M], b_{g}^{(2)}[1], \ldots, b_{g}^{(2)}[M]\right]
\end{gathered}
$$

depending on application to uncalibrated or calibrated data. Figure 2.8 displays a flow chart illustrating the AO process with the additional estimation step.

Step

Estimate

Fixed

(1)

(2)
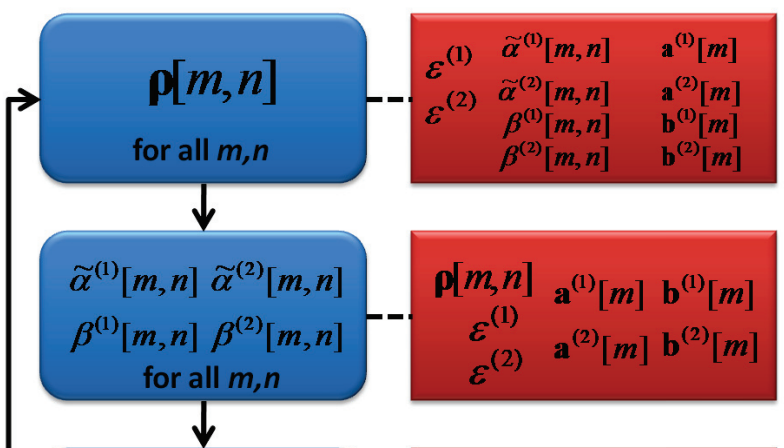

(3)

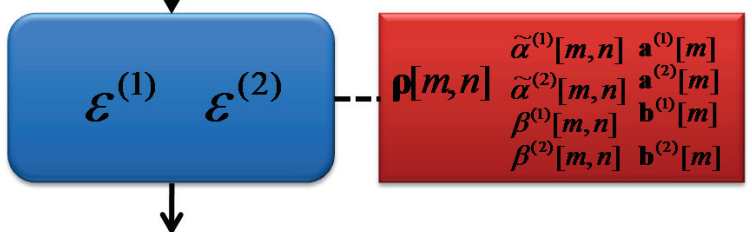

(4)

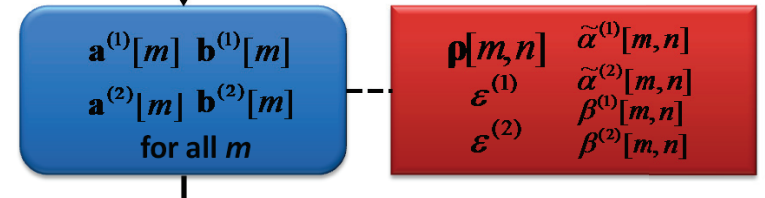

Figure 2.8: Flow chart for alternating optimization applied for model-based change detection for extended data model

In order to implement this estimation approach, a good initial estimate of the gain and offset terms are required, or a good initial estimate of reflectance vectors at each index $m$. One potential method for obtaining initial estimates involves the 
use of the NDVI discussed in Section 2.4.3. NDVI is essentially a measure of the relative increase in signal level between $0.66 \mu \mathrm{m}$ and $0.86 \mu \mathrm{m}$. The cutoff of chlorophyll absorption occurs near $0.66 \mu \mathrm{m}$ and is replaced by high reflectance resulting from scattering within the cell structure of vegetation. As a result, the NDVI will be large for healthy vegetation in the scene. Using a library vegetation reflectance $\boldsymbol{\rho}_{v e g}$, the measurement associated with vegetation for uncalibrated data is given by,

$$
\begin{aligned}
\mathbf{r}_{\text {veg }}^{(t)} & =\mathbf{a}^{(t)}[m] \odot \boldsymbol{\rho}_{\text {veg }}^{(t)} \odot\left(\tilde{\alpha}^{(t)} \mathbf{S}^{(t)} \boldsymbol{\epsilon}^{(t)}+\beta^{(t)} \mathbf{D}^{(t)} \boldsymbol{\epsilon}^{(t)}\right) \\
& +\mathbf{a}^{(t)}[m] \odot \mathbf{P}^{(t)} \boldsymbol{\epsilon}^{(t)}+\mathbf{b}^{(t)}[m]
\end{aligned}
$$

Similarly, deep shadow pixels are identified in the scene by examining those pixels with the lowest spectral norm. Deep shadows are assumed to receive no direct or diffuse illumination. Consequently, the reflectance of such a pixel is assumed to be zero without loss of generality, i.e., $\boldsymbol{\rho}_{\text {shad }}=\mathbf{0}$. The measurement associated with shadow pixels is given by,

$$
\mathbf{r}_{\text {shad }}^{(t)}=\mathbf{a}^{(t)}[m] \odot \mathbf{P}^{(t)} \boldsymbol{\epsilon}^{(t)}+\mathbf{b}^{(t)}[m]
$$

By defining,

$$
\mathbf{L}_{u-v}^{(t)}=\boldsymbol{\rho}_{v e g}^{(t)} \odot\left(\tilde{\alpha}^{(t)} \mathbf{S}^{(t)} \boldsymbol{\epsilon}^{(t)}+\beta^{(t)} \mathbf{D}^{(t)} \boldsymbol{\epsilon}^{(t)}\right)+\mathbf{P}^{(t)} \boldsymbol{\epsilon}^{(t)}
$$

and

$$
\mathbf{L}_{u-s}^{(t)}=\mathbf{P}^{(t)} \boldsymbol{\epsilon}^{(t)}
$$

the vegetation and shade measurements are given by,

$$
\mathbf{r}_{v e g}^{(t)}=\mathbf{a}^{(t)}[m] \odot \mathbf{L}_{u-v}^{(t)}+\mathbf{b}^{(t)}[m]
$$

and

$$
\mathbf{r}_{\text {shad }}^{(t)}=\mathbf{a}^{(t)}[m] \odot \mathbf{L}_{u-s}^{(t)}+\mathbf{b}^{(t)}[m]
$$


The sensor gain and dark current offset terms are then given by,

$$
a^{(t)}\left[\lambda_{k}\right]=\frac{r_{v e g}^{(t)}\left[\lambda_{k}\right]-r_{s h a d}^{(t)}\left[\lambda_{k}\right]}{L_{u-v}^{(t)}\left[\lambda_{k}\right]-L_{u-s}^{(t)}\left[\lambda_{k}\right]}
$$

and

$$
b^{(t)}\left[\lambda_{k}\right]=\frac{r_{s h a d}^{(t)}\left[\lambda_{k}\right] L_{u-v}^{(t)}\left[\lambda_{k}\right]-r_{v e g}^{(t)}\left[\lambda_{k}\right] L_{u-s}^{(t)}\left[\lambda_{k}\right]}{L_{u-v}^{(t)}\left[\lambda_{k}\right]-L_{u-s}^{(t)}\left[\lambda_{k}\right]}
$$

In practice, estimates of the upwelling radiance for the vegetation and shadow must be used, $\hat{\mathbf{L}}_{u-v}^{(t)}$ and $\hat{\mathbf{L}}_{u-s}^{(t)}$, which are determined by the initial estimates of the basis coefficients, shadow coefficients, and the vegetation reflectance used. Note that in most cases, it will be extremely difficult to identify a vegetation pixel and a deep shadow pixel in every row $(m=1, \ldots, M)$. Consequently, one must use the same estimate of the detector gain and dark current offset for all rows initially, i.e., $\mathbf{a}^{(t)}[m]=$ $\mathbf{a}^{(t)}$ and $\mathbf{b}^{(t)}[m]=\mathbf{b}^{(t)}$. Ignoring the spatial dependence of the gain and offset may suffice for initial estimates wherein later iterations of the $\mathrm{AO}$ can incorporate the spatial dependence.

The same NDVI derivations apply for relatively calibrated data, where in this case $\mathbf{a}_{g}^{(t)}$ replaces $\mathbf{a}^{(t)}$ and $\mathbf{b}_{g}^{(t)}$ replaces $\mathbf{b}^{(t)}$. Additionally, the estimation problem can be simplified assuming the dark current offset was sufficiently removed during the calibration process. In this case, $\mathbf{b}_{g}^{(t)} \approx 0$ as discussed earlier. As a result, the initial estimate of the offset is $\hat{\mathbf{b}}_{g}^{(t)}=0$ and the initial estimate of the gain can be obtained directly from a shadow measurement by

$$
a_{g}^{(t)}\left[\lambda_{k}\right]=\frac{r_{\text {shad }}^{(t)}\left[\lambda_{k}\right]}{L_{u-s}^{(t)}\left[\lambda_{k}\right]}
$$

which greatly simplifies the initial estimation problem.

Several additional notes must be made in regard to the validity and applicability of this model for airborne data. Figure 2.7(a) displays how a pushbroom imaging 
spectrometer ideally collects imagery. However, in practice, the aircraft rolls and tilts while collecting imagery. The majority of airborne data must be geocorrected to compensate for the roll and tilt of the aircraft. This roll manifests itself in the uncorrected imagery as warping in the spatial structures of the scene. Straight roads appear curvy and other spatial structures appear warped. The geocorrection remaps the pixels in the scene to correct for this warping. Figure 2.9 displays an example of geocorrection applied to airborne imagery. The geocorrection is typically applied
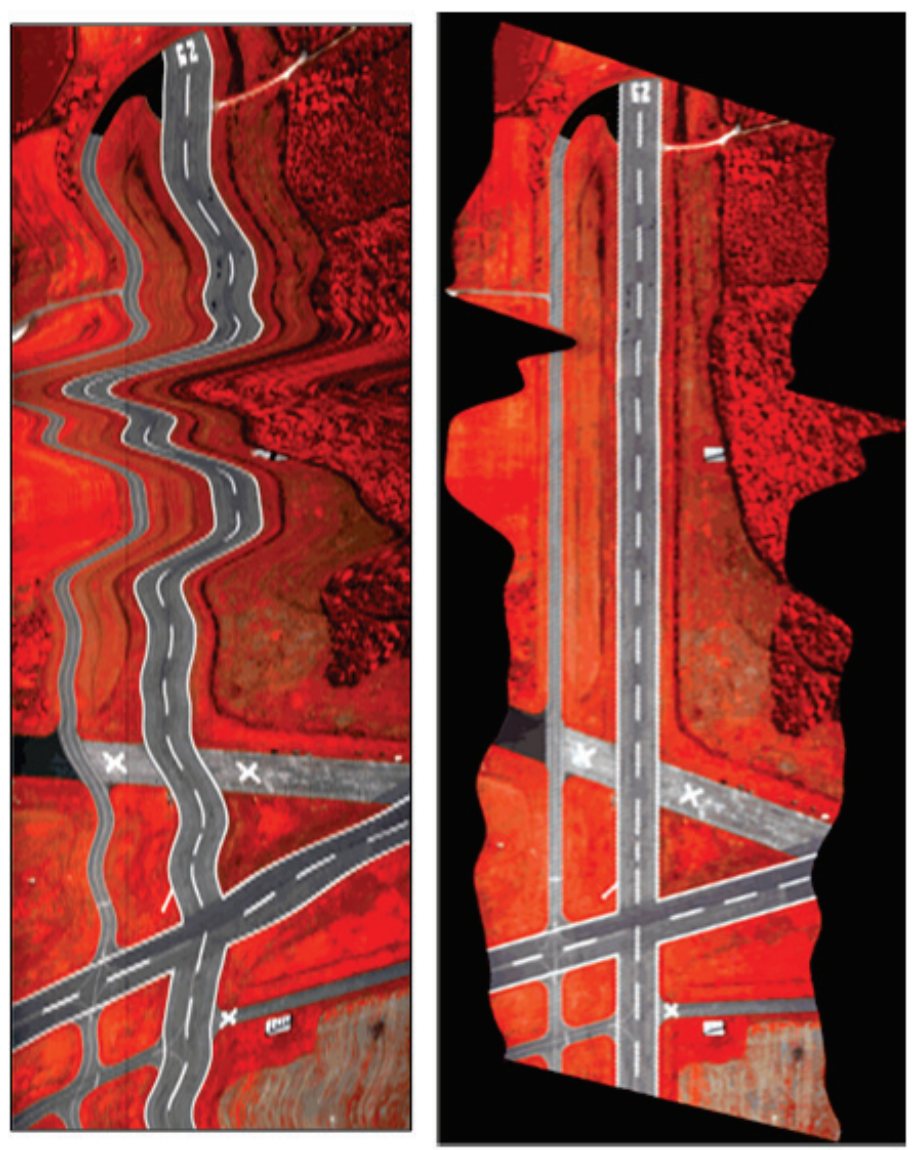

Figure 2.9: Example of geocorrection applied to airborne imagery to correct rolling of aircraft during collection 
after the data has been calibrated. As a result, in the geocorrected imagery, the assumption that the gain and offset terms are a function of only one spatial index may be invalid. In the example images provided in Figure 2.9, the same gain and offset applies along all pixels in a column of the original uncorrected imagery. When the geocorrection has been applied, the same dependence no longer holds. The warping is more significant for certain portions of the image than others. In regions where the warping is minimal, the assumption may still hold true. From previous analysis, the variation of the detector gain and offset varies slowly as a function of the spatial index. Consequently, a change in several rows as a result of the geocorrection may not be significant in cases of small warping. For significant warping, an alternative method must be determined. If the transformation applied to the spatial positions is known, one can potentially use this knowledge to determine the spatial dependence of the gain and offset terms. 


\section{Chapter 3: HYPERSPECTRAL NOISE ESTIMATION}

\subsection{Introduction}

The model-based approach to the change detection problem requires an estimate of the noise covariance matrix $\boldsymbol{\Gamma}^{(t)}[m, n]$. Noise estimation for hyperspectral data is a complex problem with more general application in hyperspectral data exploitation. In order to effectively exploit hyperspectral data, an appropriate data model must be used to describe the variability of the data, which ultimately requires an estimate of the noise. Data exploitation tasks, such as dimensionality reduction, classification, target detection, anomaly detection, and unmixing all require appropriate data models $[49,78,47,50]$. The data models used for these tasks are complicated by the fact that variability in hyperspectral imagery arises from two fundamentally different processes: sensor noise and scene variability. Kerekes provides an extensive end-toend model for hyperspectral data that explains the terms contributing to the scene variability [46].

In the applications above, the combined sensor noise and scene variability is often characterized using the estimated sample covariance matrix of the scene, which is then used in the respective data models and exploitation tasks. Gaussian mixture models are often used to account for the presence of multiple material classes 
within the scene $[80,30]$. In many cases, the variability of the data is driven primarily by the variability associated with the spatial texture and material diversity and the sample covariance matrix can provide a good description of this variability when using mixture models. However, in other applications an estimate of the variability due solely to the sensor noise component is desirable. Green discusses a maximum noise fraction (MNF) transform that requires an estimate of the noise covariance [36]. The MNF is very similar to the reduced rank filtering (RRF) method described in the work of Morillo-Contreras, which also requires a noise estimate [57]. Additionally, applications that compare temporal hyperspectral measurements, such as the model-based change detection problem, require knowledge of the sensor noise characteristics. Here, the problem and challenges associated with estimating hyperspectral sensor noise characteristics for pushbroom and whiskbroom imaging type hyperspectral systems is discussed.

In order to understand the characteristics of the noise due to the sensor, one must understand the noise sources associated with the detector array and readout electronics, and how these noise components are impacted by the calibration routines typically applied to raw hyperspectral data. This work focuses on silicon detector array cameras used in the visible/near-infrared (VNIR) spectral range, for which the literature on noise is extensive. In general, visible cameras have a signal-dependent noise component and a signal-independent noise component that add independently $[39,38,76,40,14,26,48]$. The signal-dependent component, commonly referred to as shot or photon noise, results from the random nature of the photon arrival/detection process. The signal-independent components result from sensor electronics and quantization noise. The characteristics of these noise sources are transformed by 
calibration routines applied to the data. In particular, non-uniformity correction is applied to the sensed data to remove pattern noise present as discussed in Section 2.6. Additionally, spectral binning or filtering may be applied to improve signal-to-noise ratio (SNR). Both of these processes alter the statistics of the noise terms.

Healey provides an in-depth discussion of the noise sources of visible cameras and calibration procedures that are typically applied to broadband imagery [39, 38]. In this work, Healey provides a description of the image noise and how the calibration impacts these noise terms. He subsequently develops a linear model that describes the noise variance of the calibrated data as a function of signal level. He additionally provides a method for determining the gain and offset terms for this linear model using controlled light sources. However, this work has not directly been extended to hyperspectral data sets.

While Healey provides a method for characterizing the camera noise in the laboratory using calibration sources, one would like to be able to estimate the noise using in-scene data for situations where laboratory characterization cannot be done. In many cases, noise is estimated in a supervised fashion from spatially uniform calibration panels intentionally placed within the imagery for system analysis. As a result of their uniformity, any variability in measurements from pixel to pixel is assumed to result from sensor noise. Nischan discusses the use of a calibration panel within HYDICE hyperspectral imagery to estimate noise [62]. However, this approach may not incorporate the signal-dependence of the noise if the noise is analyzed at only the single illumination level dictated by the reflectance of the calibration panel. Suen and Healey make use of the multiple gray-level calibration panels in HYDICE images to 
estimate a gain and offset describing the noise variance as a function of signal-level [83].

One of the limitations with the supervised approach using uniform areas such as calibration panels is the difficulty in assessing the texture associated with the pixels. Calibration panels can often be weathered from use in the field and may lack the desired spatial uniformity. In many cases, a user must manually identify spatial pixels which appear to be uniform; however, the variance associated with the measurements may be greater than the expected noise of the system due to texture and spatial non-uniformity. Aiazzi describes a window-based method for estimating hyperspectral noise variance that explicitly tries to identify spatially uniform regions in an automated fashion [1]. In this work, he computes the sample mean and sample variance in a small window around each pixel, and creates a scatter plot of the estimated variance versus mean for each band separately. He fits a horizontal line through the lower region of the scatter plot; the y-intercept of this line corresponds to the noise variance for that particular band. However, this approach assumes a signal-independent noise variance which fails to address the true signal dependence of the noise.

Gao et. al. discuss an automated algorithm for estimating noise which initially uses an "object seeking" algorithm that attempts to segment all pixels into object classes [34]. Linear regression is then applied to each object class separately and the residual error associated with the regression is assumed to be noise. However, this model also assumes a signal-independent noise. Additionally, the similarity metric used often has difficulty differentiating subtle class differences when classifying pixels. Martin-Herrero builds on to this work and offers an improved method for classification 
of the pixels into classes [52], but this also fails to address the signal dependence and spatial dependence of the noise statistics.

Green introduces a noise estimation technique in his work with the MNF transform discussed earlier [36]. Green assumes a signal plus noise model for multi-spectral data, both of which are stochastic. The noise covariance is estimated using neighboring pixel differences under the assumption of strong spatial correlation in the signal versus weak spatial correlation of the noise. By differencing, the signal component is removed, leaving only a noise component whose variance is twice that of the true noise. This method is subject to error when a significant amount of texture exists in the scene. Additionally, the model does not explicitly incorporate the signal-dependence or spatial dependence of the noise.

An alternate method for estimating the sensor noise was developed for use on AVIRIS hyperspectral data. In this work, Roger discusses a multiple regression model to describe the data [69]. Essentially, the regression model assumes the spectral measurement of a spatial pixel at a particular band can be described as a linear combination of the measurements of the same spatial pixel at the immediately adjacent bands and the neighboring pixel at the same band. Separate regression coefficients are estimated for small, non-overlapping blocks of pixels. After estimating the regression coefficients, the residual error in the regression model is attributed to noise. The sample variance of each block is computed and a statistical method is applied to the sample estimates generated for all the blocks to select a homogeneous set to average as the noise variance for that particular band. Bioucas-Diaz utilizes a similar regression model, but models the measurement at a particular band as a linear combination of all the remaining bands rather than simply the adjacent bands [11]. 
These methods are used primarily in developing signal-independent noise estimates. However, the approaches potentially can be expanded to develop a signal-dependent noise estimate.

In this chapter, a comprehensive model of HSI sensor noise and associated methods to estimate the model parameters from measured data are provided. While the previous work considered above addresses components needed in a realistic model, none of the approaches are complete. Ultimately, the noise estimation techniques described suffer from difficulty in identifying spatially uniform areas in the scene, ignore spatial and spectral dependences of the noise, and/or fail to identify the signal-dependence of the noise. Healey provides a more complete and accurate model for the noise terms and estimation of this noise for broadband imagery. However, this work has not been directly extended to hyperspectral data. The model presented here extends Healey's work in modeling camera noise in hyperspectral data sets. In particular, the gain and offset term developed in Healey's work will have spatial and spectral dependences associated with them, which result from the calibration process. The previous chapter provided information about calibration of hyperspectral data. In Section 3.2, the calibration techniques will be briefly reviewed in regard to the noise estimation problem. In Section 3.3, the theoretical distribution of uncalibrated and calibrated hyperspectral noise is discussed. Finally, in Section 3.4, techniques for noise estimation using calibration data or from in-scene data via supervised and unsupervised methods are provided. 


\subsection{Hyperspectral Data and Calibration}

The noise statistics associated with hyperspectral data are unique not only because of the calibration routines applied but also because of the manner in which the data is collected. Here, the focus is given to noise characteristics of VNIR-SWIR pushbroom hyperspectral imaging systems, such as that displayed in Figure 2.7(a), using photonic detector technology. In general, the results can be extended for similar hyperspectral imaging sensors.

Many sources exist that discuss the various noise components associated with photonic array camera systems $[38,76,40,14]$. From (2.77), the raw, uncalibrated hyperspectral data are related to the incoming radiance by the linear model,

$$
r[m, n, k]=a[m, k] L[m, n, k]+b[m, k]+\nu_{s}[m, n, k]+\nu_{i}[m, n, k],
$$

where $\nu_{s}[m, n, k]$ is a zero-mean, signal-dependent noise with variance dependent on $L[m, n, k]$, and $\nu_{i}[m, n, k]$ is a zero-mean, signal-independent noise. Ultimately, one would like to estimate the total distribution associated with these noise sources in order to accurately model hyperspectral data. However, the calibration process used to remove dark current and PRNU ultimately impacts the noise statistics of the raw data.

From (2.78), the linear calibration applied to raw imagery is given by,

$$
c[m, n, k]=\frac{r[m, n, k]-b_{c}[m, k]}{a_{c}[m, k]},
$$

For the moment, assume that an absolute radiometric calibration is performed, such that the NUC corrected data is given by,

$$
\hat{L}[m, n, k]=\frac{r[m, n, k]-\hat{b}[m, k]}{\hat{a}[m, k]},
$$


which is described similarly by Schott [76]. While residual non-uniformity can exist as a result of the nonlinear response of the detectors, the assumption is made that the residual error is relatively small in comparison to other noise terms existing in the system.

After performing the NUC, the hyperspectral data may be filtered either spatially or spectrally to improve SNR. Here the case is examined in which the data is filtered spectrally by,

$$
\hat{L}_{f}[m, n, k]=\sum_{l=-\left(N_{f}-1\right) / 2}^{\left(N_{f}-1\right) / 2} h[l] \hat{L}[m, n, k-l],
$$

where $N_{f}$ is the odd filter length and $h$ are the respective filter coefficients. After filtering spectrally, the data is often downsampled spectrally to reduce the number of bands which no longer provide additional information as a result of the reduction in spectral resolution. Each step of the calibration process will impact the noise characteristics.

\subsection{Theoretical Noise Model}

A linear noise model is developed describing the expected variance of the calibrated hyperspectral data based upon the uncalibrated noise model and the calibration steps applied. The linear model describes the variance as a direct function of the signal collected by the sensor.

\subsubsection{Uncalibrated Data Noise Model}

The number of detected photoelectrons $N_{p}$ for a given integration time can be modeled as a Poisson random process [38] for which the variance of the noise is equal to the mean of the signal. This shot noise has a variance $\sigma_{N_{p}}^{2}$ equal to the mean 
detected photoelectrons as given in (2.74), i.e.,

$$
\sigma_{N_{p}}^{2}[m, n, k]=\mu_{N_{p}}[m, n, k]
$$

The associated shot noise variance for $\nu_{s}$ of the uncalibrated data model given in (2.77) is then given by,

$$
\begin{aligned}
\sigma_{s}^{2}[m, n, k]=\operatorname{var}\left(\nu_{s}[m, n, k]\right) & =g^{2}[m, k] \sigma_{N_{p}}^{2}[m, n, k] \\
& =g^{2}[m, k] \mu_{N_{p}}[m, k] \\
& =g[m, k]\left(\mu_{R}[m, n, k]-b[m, k]\right),
\end{aligned}
$$

with $\mu_{R}[m, n, k]=a[m, k] L[m, n, k]+b[m, k]$. Following from this, the total noise variance for the uncalibrated data is given by,

$$
\begin{aligned}
\sigma_{R}^{2}[m, n, k] & =\sigma_{s}^{2}[m, n, k]+\sigma_{i}^{2}[m, k] \\
& =g[m, k]\left(\mu_{R}[m, n, k]-b[m, k]\right)+\sigma_{i}^{2}[m, k],
\end{aligned}
$$

where

$$
\operatorname{var}\left(\nu_{i}[m, n, k]\right)=\sigma_{i}^{2}[m, k]
$$

resulting in a noise model for the uncalibrated data. Note, in general, the variance of the signal-independent noise does not depend on the frame index $n$.

\subsubsection{Calibrated Data Noise Model}

The application of the NUC and spectral filtering will alter the noise variance and associated linear noise model. Using (3.1), the variance of the NUC data is given by,

$$
\sigma_{\hat{L}}^{2}[m, n, k]=\frac{1}{\hat{a}^{2}[m, k]} \sigma_{R}^{2}[m, n, k] .
$$


Using the linear model given in (3.5), the model describing the calibrated data is given by,

$$
\begin{aligned}
\sigma_{\hat{L}}^{2}[m, n, k] & =\frac{1}{\hat{a}^{2}[m, k]}\left(g[m, k]\left(\mu_{R}[m, n, k]-b[m, k]\right)+\sigma_{i}^{2}[m, k]\right) \\
& =\frac{g[m, k]}{\hat{a}[m, k]} L[m, n, k]+\frac{\sigma_{i}^{2}[m, k]}{\hat{a}^{2}[m, k]}
\end{aligned}
$$

where it is assumed that $\hat{a}[m, k] \approx a[m, k]$ and $\hat{b}[m, k] \approx b[m, k]$.

Finally, the linear model for the spectrally filtered data is examined. Using (3.2), the variance of the filtered data is given by,

$$
\sigma_{\hat{L}_{f}}^{2}[m, n, k]=\sum_{l=-\left(N_{f}-1\right) / 2}^{\left(N_{f}-1\right) / 2} h^{2}[l] \sigma_{\hat{L}}^{2}[m, n, k-l] .
$$

assuming statistical independence of the spectral measurements. If the filter length is relatively small, one can assume the noiseless components of the signals being filtered will be approximately equal because they are neighboring spectral bands,

$$
\hat{L}[m, n, k-l] \approx \hat{L}[m, n, k], \quad-\left(N_{f}-1\right) / 2 \leq l \leq\left(N_{f}-1\right) / 2
$$

and the variance of the random variables being summed will be approximately equal. By defining the sum and sum-squared of the filter coefficients as,

$$
S_{f}=\sum_{l=-\left(N_{f}-1\right) / 2}^{\left(N_{f}-1\right) / 2} h[l]
$$

and

$$
P_{f}=\sum_{l=-\left(N_{f}-1\right) / 2}^{\left(N_{f}-1\right) / 2} h^{2}[l]
$$

one can approximate the filtering by

$$
\hat{L}_{f}[m, n, k] \approx S_{f} \hat{L}[m, n, k],
$$


with the variance of the filtered data then given by

$$
\sigma_{\hat{L}_{f}}^{2}[m, n, k] \approx P_{f} \sigma_{\hat{L}}^{2}[m, n, k]
$$

Substitution using (3.7) results in,

$$
\sigma_{\hat{L}_{f}}^{2}[m, n, k]=\frac{P_{f} g[m, k]}{S_{f} \hat{a}[m, k]} L_{f}[m, n, k]+\frac{P_{f}}{\hat{a}^{2}[m, k]} \sigma_{i}^{2}[m, k]
$$

where $L_{f}$ is the filtered data, which ultimately provides the linear model for the calibrated data. This can be written as a more general linear model given by

$$
\sigma_{\hat{L}_{f}}^{2}[m, n, k]=a_{\hat{L}_{f}}[m, k] L_{f}[m, n, k]+b_{\hat{L}_{f}}[m, k],
$$

where

$$
a_{\hat{L}_{f}}[m, k]=\frac{P_{f} g[m, k]}{S_{f} \hat{a}[m, k]}
$$

and

$$
b_{\hat{L}_{f}}[m, k]=\frac{P_{f}}{\hat{a}^{2}[m, k]} \sigma_{i}^{2}[m, k]
$$

The noise model gain $a_{\hat{L}_{f}}[m, k]$ and offset $b_{\hat{L}_{f}}[m, k]$ are independent of frame index $n$ due to the method of collection and calibration. All of the parameters in this data model are known from the calibration and processing parameters except $g[m, k]$ and $\sigma_{i}^{2}[m, k]$, which are both system characteristics. Consequently, if one can obtain estimates of these parameters, one can characterize the noise in the calibrated data. However, a user who has no knowledge of the calibration process may need to estimate the composite gain $a_{\hat{L}_{f}}$ and offset $b_{\hat{L}_{f}}$ from the imagery itself.

\subsubsection{Spectral Correlation Characteristics}

In addition to analyzing the expected signal-dependence of the noise variance, one must understand how this signal-dependence affects the spectral correlation of 
the noise. The spectral correlation of the uncalibrated data $r$ given in $(2.77)$ is examined first. The $K$-dimensional uncalibrated measurement vector $\mathbf{r}$ at pixel $(m, n)$ is described by,

$$
\mathbf{r}=\boldsymbol{\mu}_{R}+\boldsymbol{\nu}_{s}+\boldsymbol{\nu}_{i}
$$

where

$$
\begin{aligned}
\boldsymbol{\mu}_{R}=[a[m, 1] L[m, n, 1] & +b[m, n, 1], \ldots, a[m, K] L[m, n, K]+b[m, n, K]]^{T} \\
\boldsymbol{\nu}_{s} & =\left[\nu_{s}[m, n, 1], \ldots, \nu_{s}[m, n, K]\right]^{T} \\
\boldsymbol{\nu}_{i} & =\left[\nu_{i}[m, n, 1], \ldots, \nu_{i}[m, n, K]\right]^{T}
\end{aligned}
$$

where the indices $(m, n)$ have been omitted for notational simplicity. Assuming a modestly large number of detected photons, the Poisson random process producing the signal-dependent noise $\boldsymbol{\nu}_{s}$ can be approximated by a Gaussian random process $\tilde{\boldsymbol{\nu}}_{s}$ with a signal-dependent variance,

$$
\tilde{\boldsymbol{\nu}}_{s}=\mathrm{D}_{s} \boldsymbol{\nu}
$$

where

$$
\boldsymbol{\nu} \sim \mathcal{N}(\mathbf{0}, \mathbf{I})
$$

and

$$
\mathbf{D}_{s}=\operatorname{diag}\left\{g[m, 1]\left(\mu_{R}[m, n, 1]-b[m, 1]\right), \ldots, g[m, K]\left(\mu_{R}[m, n, K]-b[m, K]\right)\right\}
$$

Similarly, the signal-independent noise can be modeled as a Gaussian random vector,

$$
\nu_{i} \sim \mathcal{N}\left(\mathbf{0}, \Gamma_{i}\right)
$$

where

$$
\boldsymbol{\Gamma}_{i}=\operatorname{diag}\left\{\sigma_{i}^{2}[m, 1], \ldots, \sigma_{i}^{2}[m, K]\right\}
$$


Using this model, the expected covariance of the uncalibrated data is given by,

$$
\boldsymbol{\Gamma}_{\mathbf{r}}=E\left[\left(\mathbf{r}-\boldsymbol{\mu}_{R}\right)\left(\mathbf{r}-\boldsymbol{\mu}_{R}\right)^{T}\right]
$$

This can be simplified using the measurement model to,

$$
\begin{aligned}
\boldsymbol{\Gamma}_{\mathbf{r}} & =E\left[\left(\tilde{\boldsymbol{\nu}}_{s}+\boldsymbol{\nu}_{i}\right)^{T}\left(\tilde{\boldsymbol{\nu}}_{s}+\boldsymbol{\nu}_{i}\right)\right] \\
& =E\left[\left(\mathbf{D}_{s} \boldsymbol{\nu}+\boldsymbol{\nu}_{i}\right)\left(\mathbf{D}_{s} \boldsymbol{\nu}+\boldsymbol{\nu}_{i}\right)^{T}\right] \\
& =\mathbf{D}_{s} \mathbf{I D}_{s}+\boldsymbol{\Gamma}_{i} \\
& =\mathbf{D}_{s}^{2}+\boldsymbol{\Gamma}_{i}
\end{aligned}
$$

which is a diagonal covariance matrix. Consequently, one expects the uncalibrated data to be spectrally uncorrelated but colored due to the signal-dependent noise variance. The spectral filtering applied during the calibration process can introduce spectral correlation. However, if the filter length is small and the data are downsampled after filtering, this correlation will be minimal. As a result, the calibrated data is expected to be spectrally uncorrelated and colored as well.

\subsubsection{Signal to Noise Ratio}

The signal-dependent noise model additionally allows one to define the signalto-noise ratio (SNR) of the calibrated data as a function of spatial position $[m, n]$, spectral position $k$, and signal level $L$. The SNR is defined here as,

$$
\begin{aligned}
S N R[m, n, k] & =\frac{L_{f}[m, n, k]}{\sigma_{\hat{L}_{f}}[m, n, k]} \\
& =\frac{L_{f}[m, n, k]}{\left(a_{\hat{L}_{f}}[m, k] L_{f}[m, n, k]+b_{\hat{L}_{f}}[m, k]\right)^{1 / 2}} \\
& =\frac{S_{f} L[m, n, k]}{\left(\frac{P_{f} g[m, k]}{\hat{a}[m, k]} L[m, n, k]+\frac{P_{f}}{\hat{a}^{2}[m, k]} \sigma_{i}^{2}[m, k]\right)^{1 / 2}} .
\end{aligned}
$$


This model supports trade studies to determine how to improve SNR at the expense of other system considerations such as spectral resolution, integration time, system front optics, operating temperature, cost, etc.

\subsection{Empirical Estimation of Noise Statistics}

Model parameter estimation is examined using calibration data if available and, alternatively, estimating the composite gain and offset directly from imagery if calibration data is unavailable. Calibration data and imagery for an AFRL VNIR hyperspectral imaging system is utilized here. The calibration process and data are known and available for this system. This allows for comparison of estimates of the parameters for the variance model in (3.12) derived from both the calibration data and the scene image data.

Additionally, the estimated noise parameters can be related back to specifications provided with the camera itself. The spectrometer uses a DALSA Pantera TF 1M60 monochrome area scan camera using a silicon detector array with a charge-coupleddevice (CCD) readout. The square focal plane array (FPA) contains 1024 x 1024 square pixels, each having $12 \mu \mathrm{m}$ side lengths with a $12 \mu \mathrm{m}$ pitch. The FPA is divided into two taps, a right and left half, to achieve a higher data transfer rate. With two taps, the camera is capable of obtaining and transferring data at a maximum rate of 60 frames per second (fps) with a minimum exposure time of $15.685 \mu \mathrm{s}$ with 12-bit data precision. The nominal charge capacity of a detector is 250,000 electrons. Further specifications for the camera are available in the user's manual [25]. 


\subsubsection{Estimation from Calibration Data}

In order to estimate the gain $g[m, k]$ and offset $\sigma_{i}^{2}[m, k]$ of the noise model for uncalibrated data, an integrating sphere with a tunable light level is used. The output illumination level on the sphere is adjusted by opening/closing an iris on the system to allow for the collection of a number of temporal frames at each light level. The sample mean $\hat{\mu}_{R}$ and variance $\hat{\sigma}_{R}^{2}$ for each pixel location $[m, k]$ are used to estimate the signal-dependent and -independent portions of the noise. Additionally, an estimate of the dark current offset $b[m, k]$ in the model is obtained by averaging a number of dark frames. For this testing, 18 different light levels are used to compute the sample statistics using 50 samples. Using least squares regression, the detector photoelectron conversion factor and read noise are estimated from these empirical samples. Figure 3.1 displays the fit to the data for row position $m=170$ and center wavelength position $\lambda_{k}=0.6 \mu m$, indicating the linear dependence of the variance on signal level as described in (3.5). Several points do not fit the regression line well due to the limited number of available samples used to obtain the sample statistics. These data are used to estimate the characteristic noise model terms for each row and wavelength position. Figure 3.2 displays the estimated detector photoelectron conversion $g[m, k]$, given by the least-squares line slope, and signal-independent noise variance $\sigma_{i}^{2}[m, k]$, determined by the sample variance of the dark frames, as a function of wavelength for three different row positions. The estimates for detector photoelectron conversion factor do not appear to depend on either row $m$ or wavelength $k$ position. However, a small drop in the estimated signal-independent noise variance is evident beyond $\lambda_{k}=$ $0.72 \mu \mathrm{m}$ on the focal plane. This results from the division of the focal plane into two separate taps as discussed earlier. The tap on the right half has generally lower read 


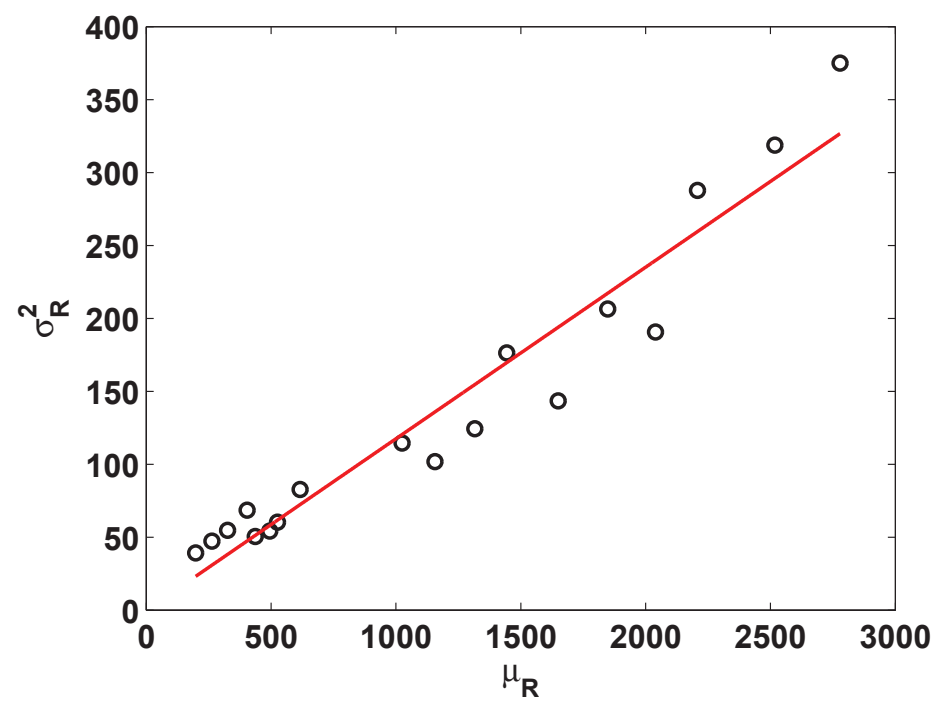

Figure 3.1: Linear fit to empirical data for noise variance at $m=170, \lambda_{k}=0.6 \mu m$

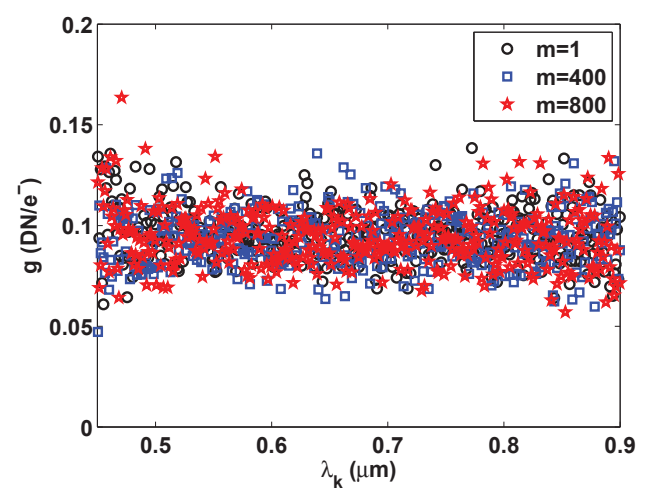

(a) Detector photoelectron conversion $\hat{g}[m, k]$

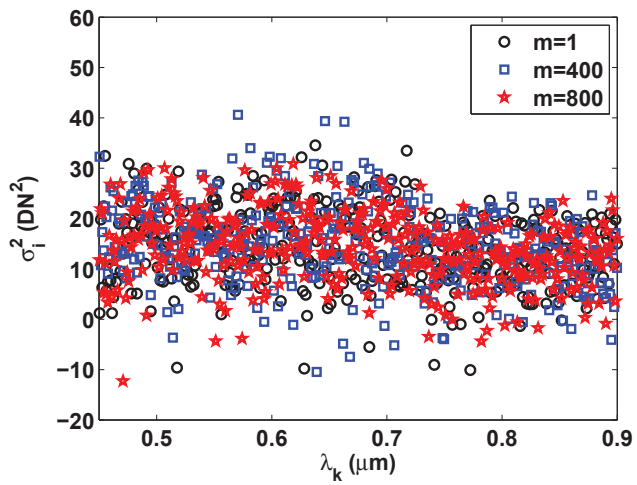

(b) Signal-independent noise variance $\hat{\sigma}_{i}^{2}[m, k]$

Figure 3.2: Estimated noise model parameters for uncalibrated noise variance given in $(3.5)$

noise than that on the left half simply due to the design and fabrication of the camera, producing this small drop. The estimates themselves have a relatively large variance 
associated with them, which results from the limited number of samples available for estimating statistics as discussed earlier. The estimates of the gain are clustered around a mean of $0.09 D N / e^{-}$. The mean read noise variance for wavelengths lower than $0.72 \mu m$ is around $15.9 D N^{2}$ and $11.3 D N^{2}$ for larger wavelengths. Consequently, the estimated model parameters are

$$
\hat{g}[m, k]=\hat{g}[k]=0.09 D N / e^{-}
$$

and

$$
\hat{\sigma}_{i}^{2}[m, k]=\hat{\sigma}_{i}^{2}[k]=\left\{\begin{array}{ll}
15.9 D N^{2} & \lambda_{k} \leq 0.72 \mu m \\
11.3 D N^{2} & \lambda_{k}>0.72 \mu m
\end{array} .\right.
$$

These estimated parameters can be used to verify specified camera performance provided by the camera manufacturer. The estimated photoelectron conversion factor corresponds to approximately 33,000 electrons producing a 3000 DN output. The system operates at well below the charge capacity because a $4 \mathrm{x}$ digital gain term is applied to amplify the low hyperspectral signal levels to the 12-bit depth of the camera. The camera specifications provide a calculated read noise variance of $1.44 D N^{2}$ when operating under the nominal gain. Due to the digital gain of 4, the expected noise variance is closer to $22 D N^{2}$, which is relatively close to the estimate derived from the data.

In many cases, a large number of different light levels of integrating sphere data to perform such a characterization may not be available. In fact, often only two light levels are available for the calibration process used to perform the NUC described earlier. Estimation of the gain and offset can still be achieved using only two light levels. However, the results will be more dependent on the quality of the sample mean and variance calculated for those two light levels. Figure 3.3 displays the estimated 
gain and offset as a function of wavelength using only two light levels. Significant

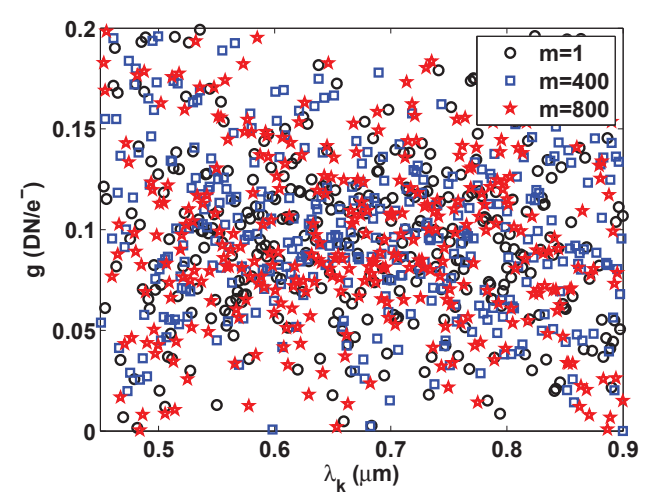

(a) Photoelectron conversion $\hat{g}[m, k]$

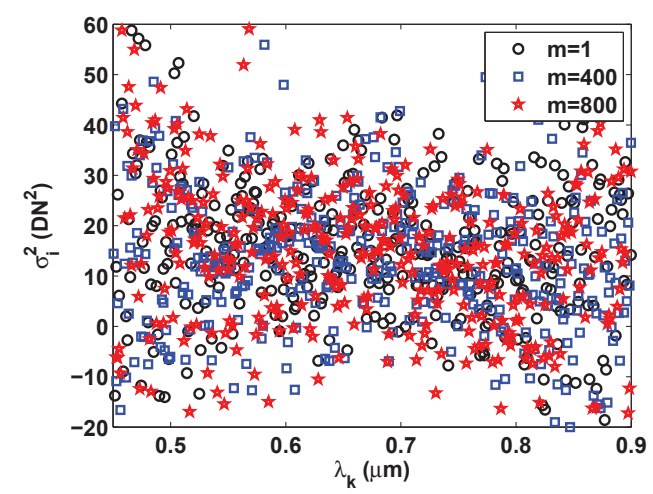

(b) Signal-independent noise variance $\hat{\sigma}_{i}^{2}[m, k]$

Figure 3.3: Estimated noise model parameters for uncalibrated noise variance given in (3.5) using only 2 light levels

variability exists in the estimated gain and offset. However, if one recognizes or knows a priori that the photoelectron gain and read noise variance will be independent of row position $m$, all the data points for a given row (i.e. $m=1, \ldots, M$ ) can be used to perform a least squares fit to estimate the model parameters at a particular wavelength position $k$. Using this approach improves the estimates as shown in Figure 3.4 .

The estimates for $g[k]$ and $\sigma_{i}^{2}[k]$ are two terms needed for the calibrated noise model given in (3.11). The only other needed information is known from the calibration process. The NUC gain term $\hat{a}[m, k]$ is determined using the known integrating sphere output along with the sample mean data $\hat{\mu}_{R}$ used in the previous step to estimate $g[k]$. The estimated gain term is shown in Figure 3.5 across the entire focal plane and for several rows for added detail. The gain term $\hat{a}[m, k]$ has both a row 


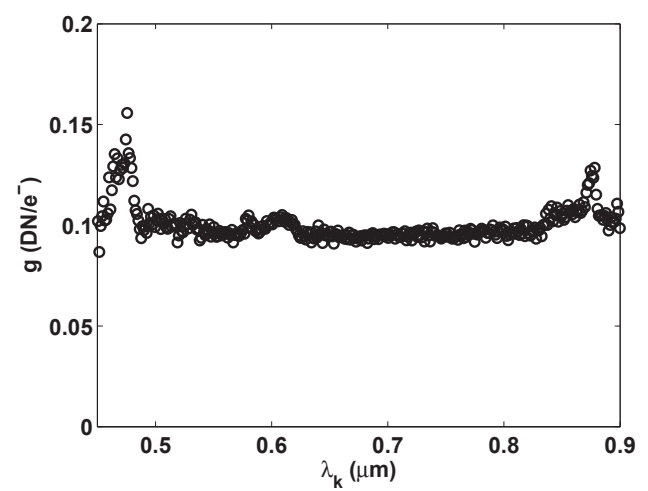

(a) Photoelectron conversion $\hat{g}[k]$

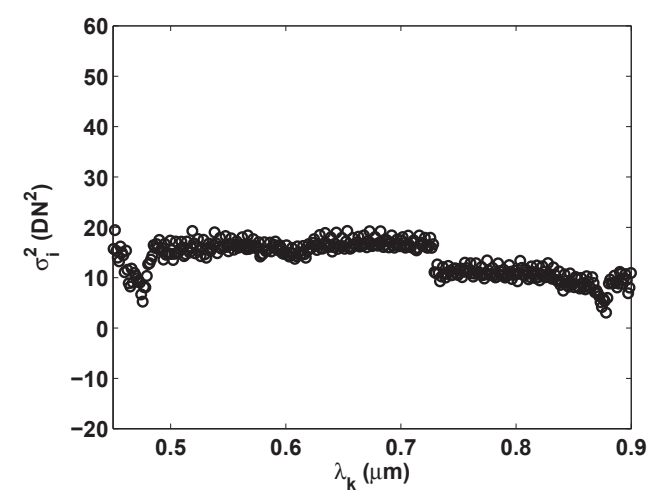

(b) Signal-independent noise variance $\hat{\sigma}_{i}^{2}[k]$

Figure 3.4: Estimated noise model parameters for uncalibrated noise variance given in (3.5) using 2 light levels while utilizing spatial independence

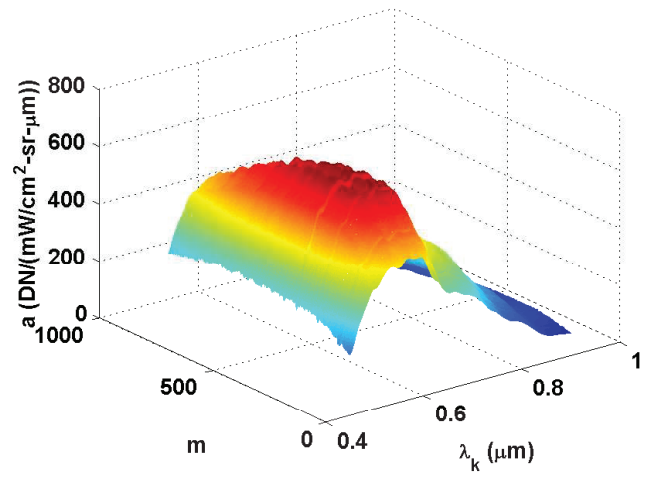

(a) Entire focal plane

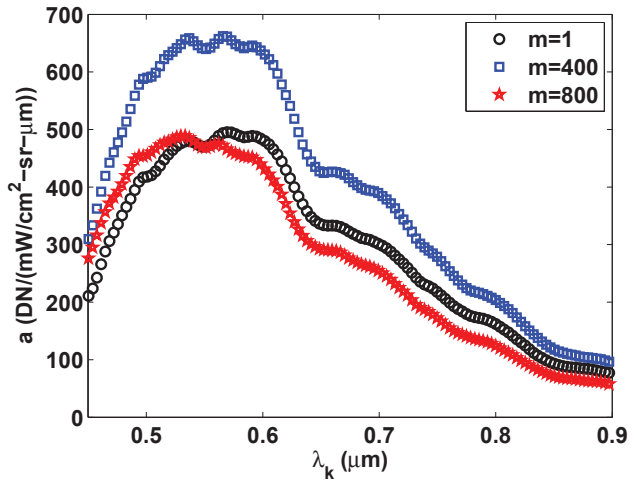

(b) Gain vs. wavelength for 3 rows

Figure 3.5: Estimated NUC gain term $\hat{a}[m, k]$ used for calibrating AFRL data.

dependence $m$ and a spectral dependence $k$. The row dependence results from system irradiance roll-off at the top and bottom of the focal plane. The spectral dependence results from the responsivity of the focal plane coupled with the spectral output of the integrating sphere used for the calibration. The DALSA 1M60 CCD digital camera 
has a silicon detector array with a responsivity curve as shown in Figure 3.6(a). The response of the detectors to different wavelengths varies significantly, with the NUC utilized to correct for this variable response. For this particular sensor and data, the integrating sphere used for the NUC has a known spectral output as shown in Figure 3.6(b). Strong correlation exists between the detector responsivity and the NUC gain

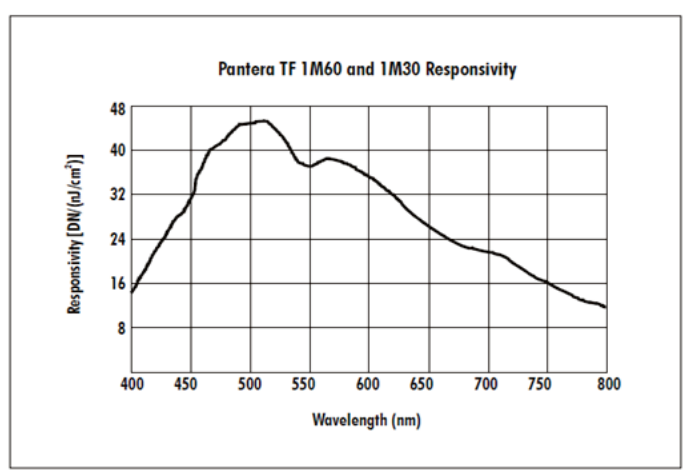

(a) Detector responsivity for AFRL in-house HSI system

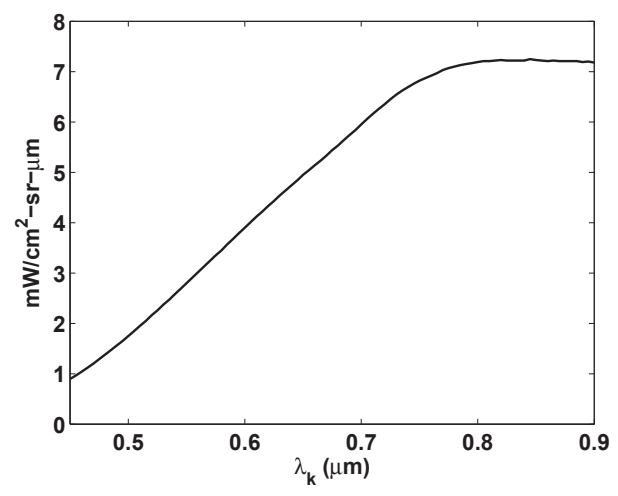

\section{(b) Spectral output of integrating sphere used for calibration}

Figure 3.6: Camera response and integrating sphere output affecting NUC gain applied

term shown in Figure 3.5.

For this data, a Kaiser spectral filter design is implemented with $S_{\text {filt }}=6.6454$ and $P_{\text {filt }}=4.8785$. Using these values along with the NUC gain term, the noise model composite gain $a_{L}[m, k]$ and offset $b_{L}[m, k]$ are computed for the calibrated data as given in (3.13) and (3.14). Figure 3.7 displays the estimated gain and offset as a function of wavelength for several rows on the detector. The results demonstrate both the spectral and spatial dependence of the noise variance model. 


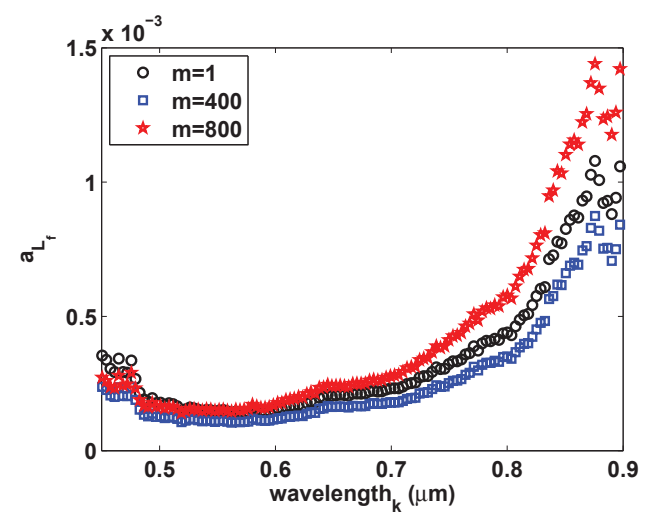

(a) Gain term $\hat{a}_{\hat{L}_{f}}$

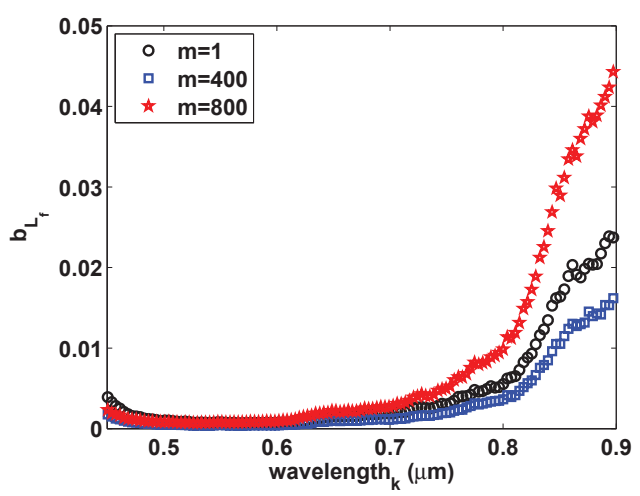

(b) Offset term $\hat{b}_{\hat{L}_{f}}$

Figure 3.7: Estimated gain and offset for noise variance model of calibrated data

After obtaining estimates of these parameters, the significance of the signal dependence can be assessed. The importance of the signal-dependent portion depends on both the signal level and the spectral band since the gain and offset vary spectrally. The fraction of the total sensor noise from the signal-independent and signaldependent terms are compared as a function of signal level $L_{f}$ for a realistic range of signal values. Figure 3.8 compares the relative fractions of the terms for three separate spectral bands across the wavelength range of the sensor. The results demonstrate that the signal-dependent noise is significant and plays an increasing role at higher signal levels.

\subsubsection{Estimation from Imagery}

After examining estimation of noise statistics using calibration data, methods are discussed for noise estimation in cases where calibration data does not exist. Both supervised and unsupervised noise estimation techniques are examined that utilize and extend concepts from the work of Aiazzi [1] and Green [36]. These estimation 


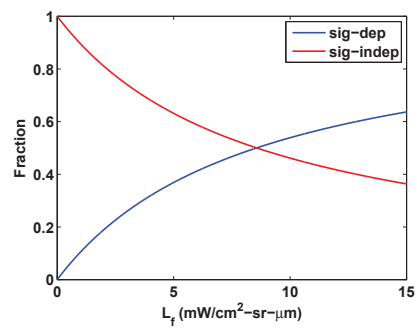

(a) $\lambda_{k}=0.45 \mu m$

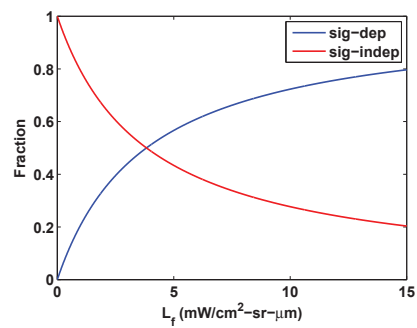

(b) $\lambda_{k}=0.57 \mu m$

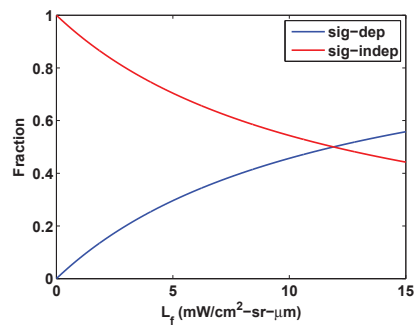

(c) $\lambda_{k}=0.81 \mu \mathrm{m}$

Figure 3.8: Comparison of fraction of total noise resulting from signal-independent and signal-dependent terms as a function of signal level

results are compared with those obtained using other estimation techniques found in the literature.

\section{Supervised Scatter Plot Method}

To begin, a supervised method for estimating the noise variance from scene imagery is examined. This approach applies a modified version of Aiazzi's work to estimate the noise model gain and offset terms [1]. The approach assumes calibration panels or other spatially uniform areas reside within the imagery. In Aiazzi's work, the hyperspectral noise is assumed to be signal-independent, which may be a good assumption if the read noise dominates the signal-dependent shot noise. However, in many cases a signal-dependent model is required as demonstrated for the AFRL data

examined here. In order to estimate $a_{\hat{L}_{f}}[m, k]$ and $b_{\hat{L}_{f}}[m, k]$, at least two estimates of $\sigma_{\hat{L}_{f}}^{2}[m, n, k]$ are required at each row position $m$ and band $k$ along with an estimate of the associated light level $L_{f}[m, n, k]$. This is impractical for real imagery. Consequently, the dependence on $m$ is assumed to be minimal in comparison with the dependence on $k$ and is ignored. 
In Aiazzi's approach, $\sigma_{\hat{L}_{f}}^{2}[k]$ and $L_{f}[m, n, k]$ of a given pixel for a particular band $k$ are estimated via the sample mean and sample variance in a small window around that pixel,

$$
\bar{L}_{f}[m, n, k]=\frac{1}{W^{2}} \sum_{w_{m}=-\frac{W-1}{2}}^{\frac{W-1}{2}} \sum_{w_{n}=-\frac{W-1}{2}}^{\frac{W-1}{2}} \hat{L}_{f}\left[m+w_{m}, n+w_{n}, k\right]
$$

and

$$
\bar{\sigma}_{\hat{L}_{f}}^{2}[m, n, k]=\frac{1}{W^{2}-1} \sum_{w_{m}=-\frac{W-1}{2}}^{\frac{W-1}{2}} \sum_{w_{n}=-\frac{W-1}{2}}^{\frac{W-1}{2}}\left(\hat{L}_{f}\left[m+w_{m}, n+w_{n}, k\right]-\bar{L}_{f}[m, n, k]\right)^{2}
$$

where $W$ is the size of the square window. The process can be generalized for other window types and sizes, a square window is used here for simplicity of notation. For areas in the scene that are spatially uniform, the sample mean provides an estimate of the noiseless signal associated with that group of pixels, whereas the sample variance provides an estimate of the noise variance of the system. For non-spatially-uniform portions of the scene, the sample variance will be much larger than the sensor noise variance. After obtaining the estimated mean and variance of each pixel, Aiazzi creates a scatter plot of the sample variance versus the sample mean for a particular band. He then argues that the bottom portion of this scatter plot (i.e., lowest sample variance pixels) will be indicative of the sensor noise variance. Due to his assumption of a signal-independent noise, he fits a horizontal line through the lower region of the scatter plot and the y-intercept of this horizontal line corresponds to the hyperspectral noise variance $\sigma_{\hat{L}_{f}}^{2}$ for that particular band. Rather than simply finding a lower bound for the scatter plot, he recognizes that the sample mean and variance are random variables. Consequently, he devises an automated method for segmenting the scatter plot into regions to help identify a lower region from which a horizontal line is fit 
through the center of the distribution. For a spatially uniform window, the sample mean is distributed as,

$$
\bar{L}_{f}[m, n, k] \sim \mathcal{N}\left(L_{f}[m, n, k], \frac{\sigma_{\hat{L}_{f}}^{2}[m, n, k]}{W^{2}}\right)
$$

and the sample variance is distributed as a scaled $\chi^{2}$ distribution with $W^{2}-1$ degrees of freedom,

$$
\frac{W^{2}-1}{\sigma_{\hat{L}_{f}}^{2}[m, n, k]} \bar{\sigma}_{\hat{L}_{f}}^{2}[m, n, k] \sim \chi^{2}\left(W^{2}-1\right),
$$

with

$$
E\left\{\bar{\sigma}_{\hat{L}_{f}}^{2}[m, n, k]\right\}=\sigma_{\hat{L}_{f}}^{2}[m, n, k]
$$

and

$$
\operatorname{var}\left\{\bar{\sigma}_{\hat{L}_{f}}^{2}[m, n, k]\right\}=\frac{2 \sigma_{\hat{L}_{f}}^{2}[m, n, k]}{W^{2}-1} .
$$

The data fitting process is repeated for each spectral band to estimate the noise variance of each band. This approach ignores both the spatial-dependence and signaldependence of the noise, which were outlined above. However, the spatial-dependence results mainly from irradiance roll-off of the system. Consequently, if the roll-off is not significant, the spatial dependence can be ignored.

A modification of Aiazzi's approach is proposed here that accounts for the signaldependence of the noise and helps with the difficulty in identifying spatially uniform regions in the scene. In this approach, the scatter plot described above is generated. However, rather than segmenting the scatter plot and fitting a horizontal line through the data, a supervised approach is employed that fits a non-horizontal line through smaller distributions of points on the scatter plot demonstrating the expected distributions associated with the sample mean and variance given in (3.27) and (3.28). After identifying these groups of pixels, a line is fit through the clusters of pixels 
whose gain and offset provide the composite gain $a_{\hat{L}_{f}}$ and offset $b_{\hat{L}_{f}}$ for the noise model above. This approach is compared with Roger's multiple-regression method [69], Green's neighbor differencing method [36], and simply using the mean and variance of manually-identified spatially-uniform regions of the scene.

The tests are performed using measured hyperspectral data of a scene collected using the AFRL VNIR sensor described and characterized above. Figure 3.9 displays a color image corresponding to the hyperspectral data cube. The hyperspectral cube

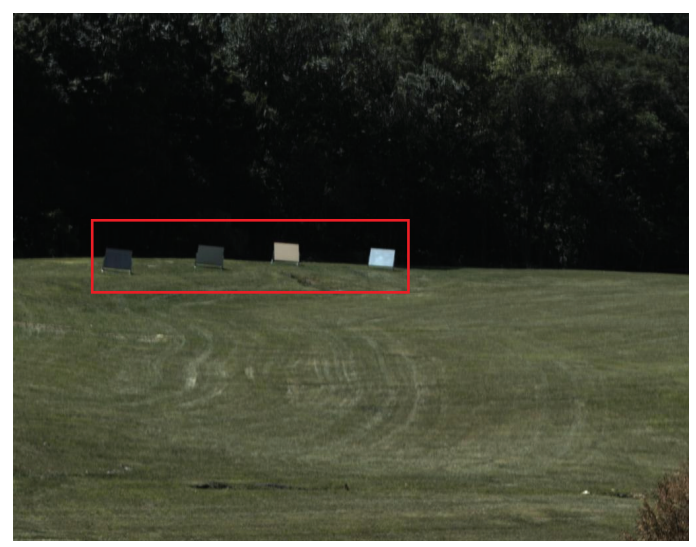

Figure 3.9: Color image of AFRL in-house hyperspectral data

has dimensions of $M=800, N=1024$, and $K=124$. Focus is given to the region outlined in the image containing the four panels (black, green, tan, and gray, from left to right) as they appear to provide the most spatially uniform regions of the scene. As mentioned earlier, one of the challenges with estimating sensor noise variance from imagery is identifying spatially uniform regions in the scene. This task is challenging because areas that may appear spatially uniform upon visual inspection may still possess texture. Consequently, the estimated variance obtained using that spatial 
region will be higher than the actual noise variance of the system. The same can be said for the panels in this scene.

Rather than manually selecting all the pixels in each panel and estimating the variance, the window approach is used with a window size smaller than the panel size. The idea is that smaller areas on each panel are more likely to be spatially uniform. This approach produces a distribution of estimates for the mean and variance associated with each panel. For spatially-uniform regions on the panels, the estimates will be distributed according to the sample mean and variance distributions provided in (3.27) and (3.28). The previous noise analysis demonstrates a noise variance that is significantly smaller than the mean signal. Consequently, the distributions present in the scatter plot are expected to be very narrow along the mean axis and a more elongated along the variance axis due to the scale of each axis. Figure 3.10(a) displays the scatter plot of the sample mean and variance obtained using a $9 \times 9$ window area around each pixel of the black, green, and tan panels for a particular band. Using the scatter plot, clusters of pixels are identified that approximate the expected distribution of the sample mean and variance. In this supervised approach, these pixels are identified manually by selecting points that visually appear to fit the expected distribution. Figure 3.11(a) displays contour plots associated with the theoretical joint-distribution of the sample mean and sample variance of each panel using $W=9, L_{f}=2.5,2.7,7.15$ and $\sigma_{\hat{L}_{f}}=0.0015,0.0016,0.0025$. The contours are extremely narrow, so a zoom on one of the contours associated with the black panel is given for added detail in Figure 3.11(b). After visually identifying the scatter plot points, a line is fit through the data using least-squares regression as demonstrated in Figure 3.10(b). The gain and offset of this linear fit are used as an estimate of $a_{\hat{L}_{f}}$ 


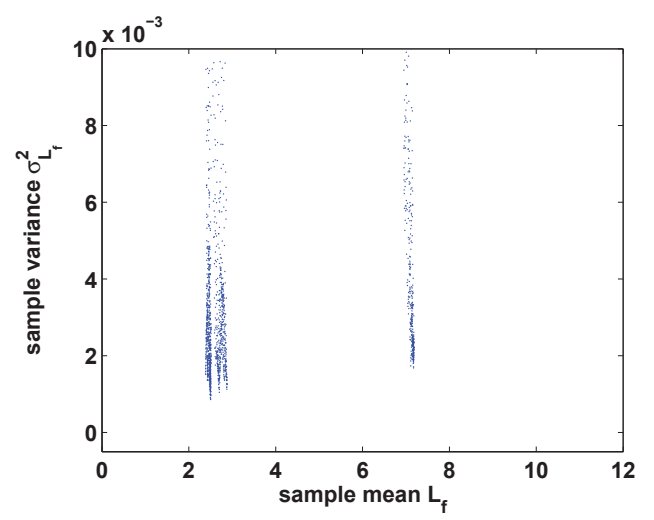

(a) Lower region of scatter plot

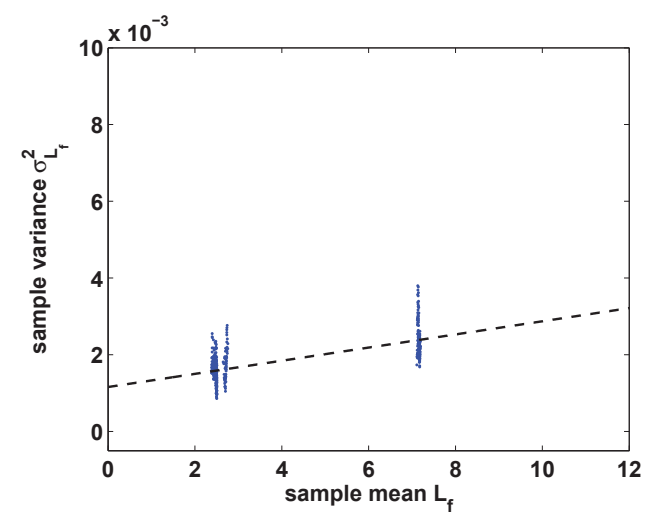

(b) Linear fit to clusters of pixels identified in scatter plot

Figure 3.10: Scatter plot of sample variance versus sample mean in small window around each pixel of the panels for a single spectral band of AFRL hyperspectral data

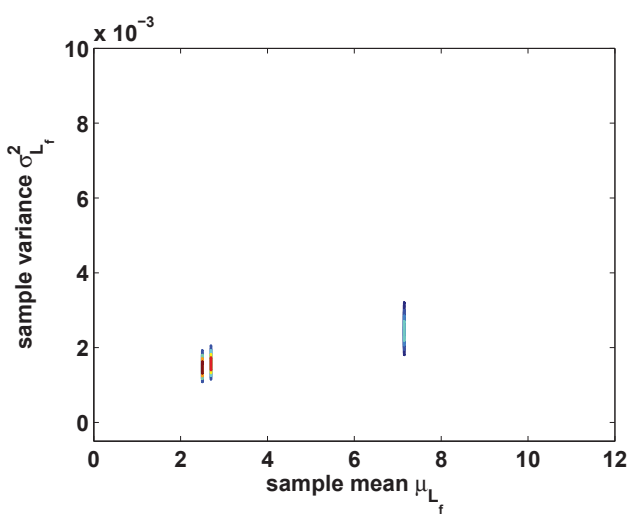

(a) All three panels

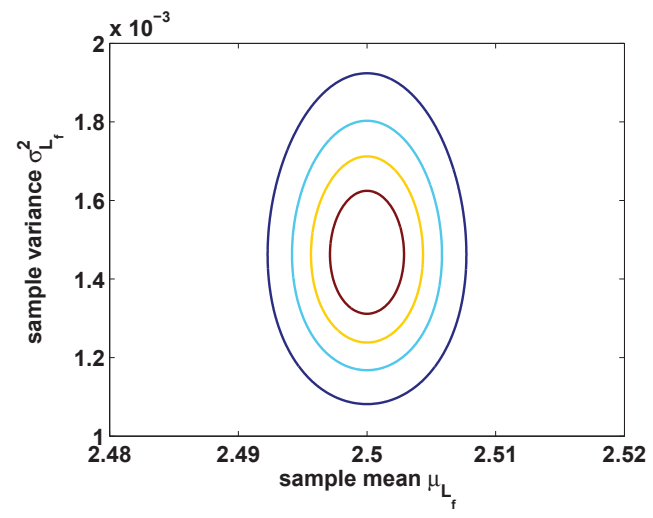

(b) Zoom on black panel contour

Figure 3.11: Contour plot of theoretical joint-distribution of sample mean and sample variance using $W=9, L_{f}=2.5,2.7,7.15$ and $\sigma_{\hat{L}_{f}}=1.5 \mathrm{e}-3,1.6 \mathrm{e}-3,2.5 \mathrm{e}-3$

and $b_{\hat{L}_{f}}$ for the given spectral band. The pixel indices associated with the selected scatter plot points are assumed to correspond to spatially uniform areas on the panels. Using these same pixel indices, a linear fit through the sample estimates for the 
remaining spectral bands is performed. Note, the spectral band used for the initial fit to determine the best pixel indices is left to the user. One can manually fit to each band separately, if so desired. As previously mentioned, the spatial dependence of $a_{\hat{L}_{f}}$ and $b_{\hat{L}_{f}}$ cannot be estimated using this method because of the limitation of the spatial positions of the panels in the scene. Estimates obtained using this scatter plot method are compared with those obtained from the calibration analysis in the previous section for a similar spatial row position $m=500$. The results are shown in Figure 3.12. The estimates correlate strongly, especially for the offset term. While the

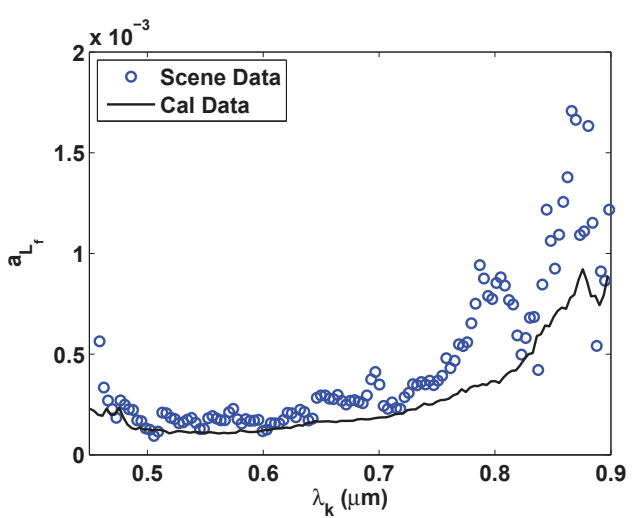

(a) Gain $\hat{a}_{L_{f}}$

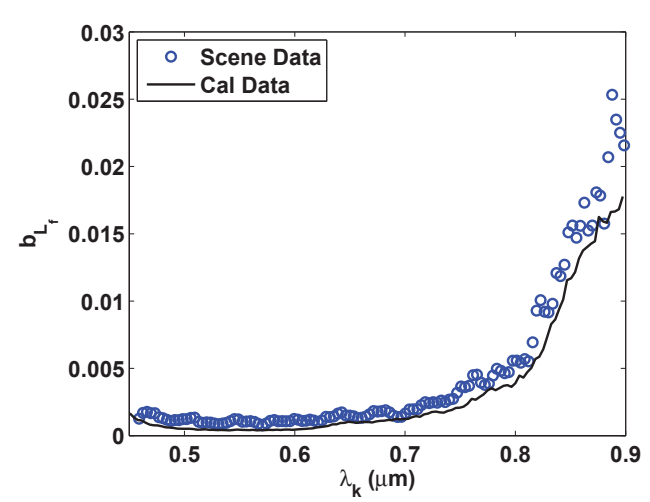

(b) Offset $\hat{b}_{L_{f}}$

Figure 3.12: Comparison of noise model gain and offset estimates obtained using calibration data and supervised scatter plot method

approach is not strictly statistically driven, the results suggest that the method offers a viable solution for estimating signal-dependent hyperspectral noise for situations where a significant number of pixels exist on each calibration panel. 


\section{Unsupervised Scatter Plot Method}

Next, an unsupervised approach is discussed that essentially attempts to perform the same functions done in the supervised approach of the previous section. In this method, the sample variance versus sample mean scatter plot is generated in the supervised method. However, neighbor difference data is used to estimate the sample variance rather than the original data. The neighbor differencing can help remove local trends between pixels, providing a better noise estimate. Additionally, these sample variance estimates can be compared with those obtained in the previous method where no neighbor differencing was used. After obtaining the scatter plot, viable points are identified within the scatter plot in an unsupervised way that were previously identified by manual inspection. This automated identification is achieved by binning the data and examining spatial relationships between the scatter plot points.

For band $k$, the scatter plot of sample variance versus sample mean is generated. The sample mean $\bar{L}_{f}[m, n, k]$ is estimated in a window of size $W$ around each pixel in the original image. The sample variance $\bar{\sigma}_{\hat{L}_{f}}^{2}[m, n, k]$ is estimated using the same size window around each pixel of the neighbor difference image rather than the original image to help suppress local trends that may exist.

The scatter plot is segmented by binning pixels using the sample mean. The minimum and maximum sample mean of the pixels is computed,

$$
\begin{gathered}
\bar{L}_{\min }[k]=\min _{m, n} \bar{L}_{f}[m, n, k] \\
\bar{L}_{\max }[k]=\max _{m, n} \bar{L}_{f}[m, n, k]
\end{gathered}
$$


Using these values, the pixels are segmented into classes using $N_{b}$ linearly spaced bins from $\bar{L}_{\min }[k]$ to $\bar{L}_{\max }[k]$. If a bin has an insignificant number of pixels, (i.e., $<1 \%$ of total number of image pixels), these pixels are grouped into the nearest neighboring bins. In this work, $N_{b}=20$ but the final number of bins meeting the population threshold tends to be around 5 for any particular band of this data. In general, the number of bins will depend on the dynamic range of intensity values for all pixels at a given band. Figure 3.13(b) displays the binning of the scatter plot.

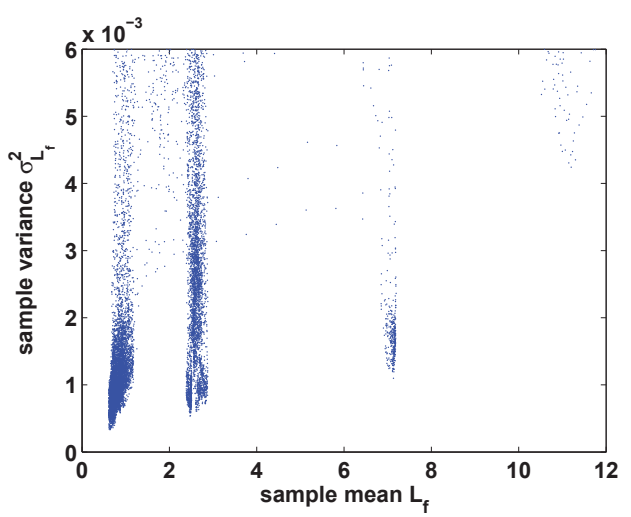

(a) Scatter plot points meeting sample variance threshold

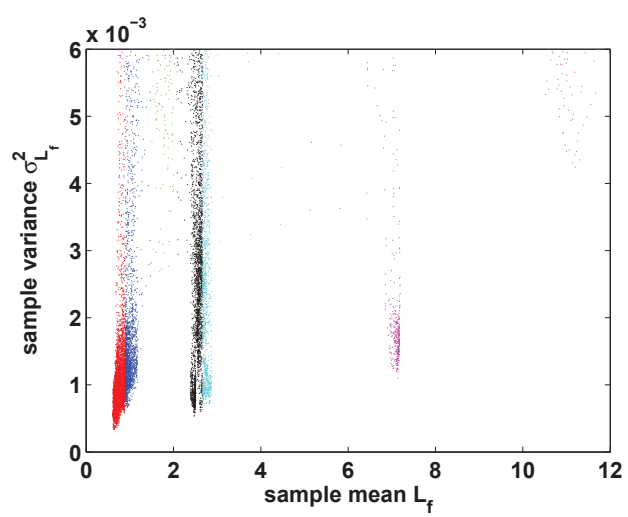

(b) Binning of data

Figure 3.13: Scatter plot of sample mean versus sample variance using neighbor difference data to estimate sample variance

For each bin, the pixels are sorted in terms of ascending sample variance. The minimum sample variance within each bin is assumed to be the true noise variance $\sigma_{\hat{L}_{f}}^{2}[m, n, k]$ for that particular mean level $L_{f}[m, n, k]$. While this noise variance tends to be less than the true noise variance, this temporary assumption provides an avenue for removing pixels with high sample variance in a statistical fashion. The cumulative distribution function $(\mathrm{CDF})$ is computed for the sample variance estimates in the 
bin using this minimum variance as the true variance and assuming the scaled $\chi^{2}$ distribution described above. Sample variance estimates in the bin for which the CDF is less than 0.999 are retained. This will remove sample variance estimates that are statistically unlikely given the assumed distribution of points. Figure 3.14(a) displays the scatter plot points retained after this step. Note the distribution of points present

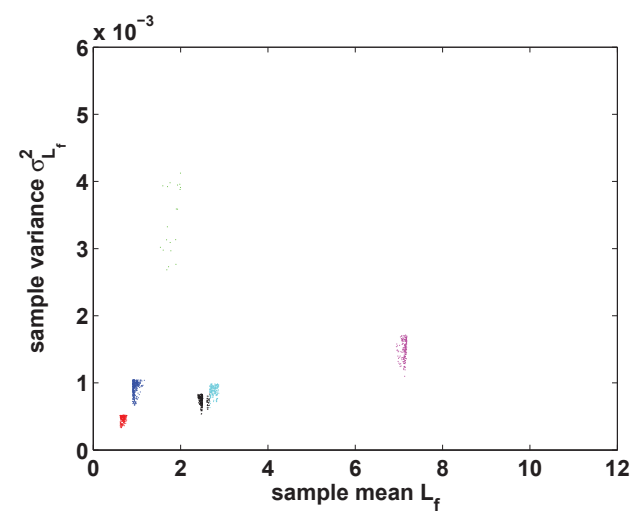

(a) Scatter plot points meeting CDF requirement

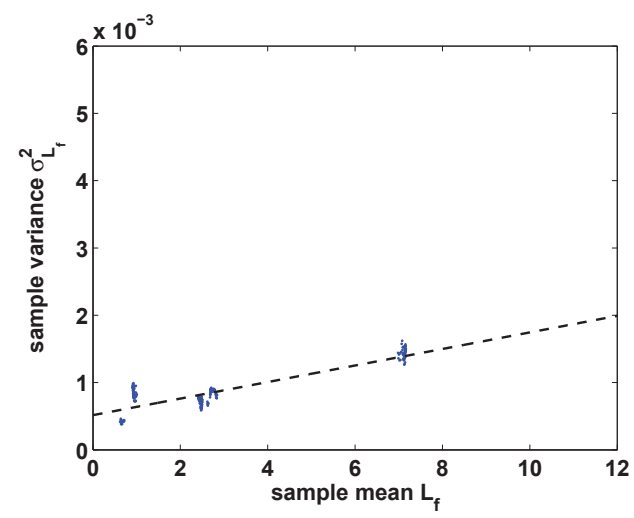

(b) Least-squares fit to data points meeting spatial neighbor requirement

Figure 3.14: Scatter plot thresholding and fit

in upper-right portion of the scatter plot in Figure 3.13(b). These points belong to the gray panel in the scene, which has visually identifiable spatial non-uniformity present resulting in a much larger sample variance. This group of pixels is removed by the CDF threshold step. Once removing sample variance estimates that are statistically unlikely, sample variance estimates are identified that make sense in a physical sense. When choosing a window size $W$ for estimating the sample variance and sample mean, the implicit assumption made by the user is that there likely exist spatially uniform areas within the scene of the same size. If such an area exists in the scene, one 
would expect the sample variance and sample mean estimated for its 8 nearest pixel neighbors to be very similar. As a result, for a given sample variance, if the sample variances of its 8 nearest spatial neighbors also exist within the same bin in the scatter plot, that point is kept. This is repeated for each of the points within a given bin kept from the previous step and for all the bins separately. Figure 3.15 displays the binning of the pixels within the scene region of interest along with the pixels identified by this thresholding process. Note that a couple of the classes (green and yellow) do not

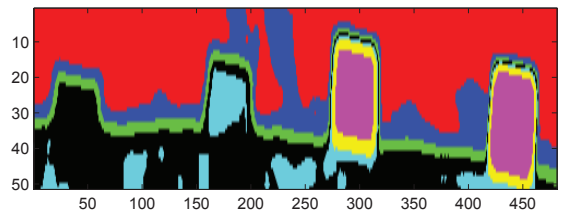

(a) Pixel bins as identified in Figure $3.13(\mathrm{~b})$

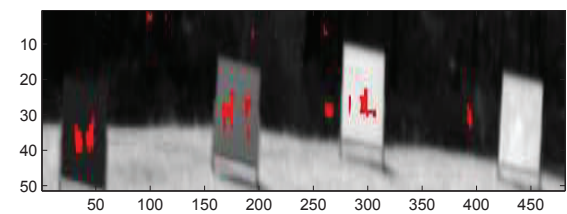

\section{(b) Pixels identified by CDF thresh-} old and neighbor comparison

Figure 3.15: Binning of pixels in scene

appear in the scatter plot simply due to the higher variance of these classes, which lie above the chosen axis limits for the figure. The points retained for line fitting come mostly from the panels. The undesired blue class in the scatter plot comes from the shadowed treeline in portions where the shadows are not as deep resulting in a larger signal. Ideally, this class would not be identified by the automated process. However, at this point classes such as these are difficult to avoid.

After determining the feasible points in the scatter plot, a linear least-squares fit is applied to the points to estimate the noise model composite gain $a_{\hat{L}_{f}}$ and offset $b_{\hat{L}_{f}}$ for the current band as shown in Figure 3.14(b), and the mean least-squares fit error 
is calculated. This procedure is repeated for each spectral band, after which the mean residual least-squares fit error for all bands are compared. Assuming the linear noise model holds true, the pixel indices producing the best linear fit (smallest mean fit error) are assumed to be associated with the most uniform areas in the scene. Using the sample variance and sample mean associated with these pixels, the gain and offset are re-calculated for each band by fitting through these points. The estimated gain and offset as shown in Figure 3.16 match very closely with the estimates obtained using calibration data. Disagreement exists between the calibration data estimates

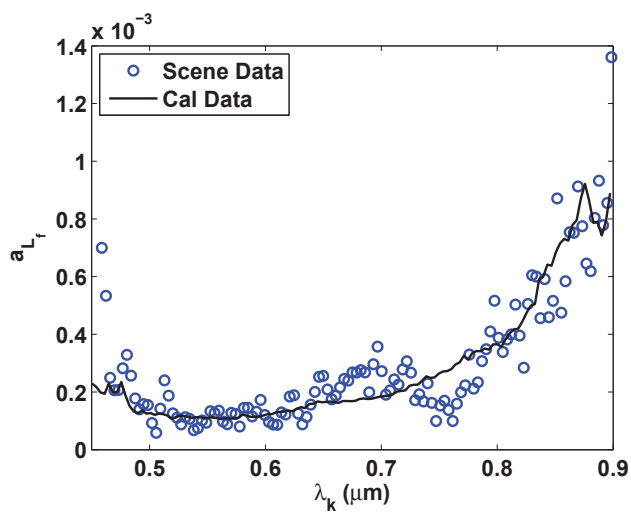

(a) Gain $\hat{a}_{L_{f}}$

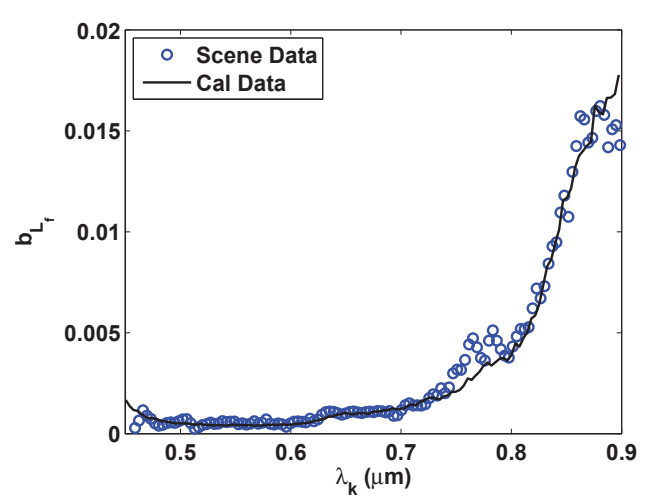

(b) Offset $\hat{b}_{L_{f}}$

Figure 3.16: Comparison of noise model gain and offset estimates obtained using calibration data and unsupervised scatter plot method

and the unsupervised estimates at small wavelengths $(<0.45 \mu m)$. This most likely results from low signal (and correspondingly low SNR) at small wavelengths for this sensor. A lack of dynamic range coupled with low SNR can produce high variability in the estimated slope and offset in the unsupervised technique. The impact of the 
estimation error at these smaller wavelengths may be insignificant as the signal in these bands is low to begin with.

The unsupervised noise estimation technique discussed here attempts to find spatially-uniform regions in the scene. While the approach uses sample statistics, a more formal Bayesian technique could potentially be developed that incorporates spatial structure. This Bayesian approach would utilize Markov random field concepts and essentially apply a hypothesis test to groups of pixels to determine if the area is spatially uniform. While this approach is a more formal development than the scatter plot method, it is more complex as well. Future work may choose to examine this option.

\section{Comparison with Other Methods in the Literature}

The noise estimation results for the two proposed methods are compared with others found in the literature and the advantages and disadvantages of each are discussed. A simple method for estimating the signal-dependent noise is to manually identify uniform regions in the scene and compute the sample mean and sample variance of each uniform region and perform linear regression on those points to compute the gain and offset. For the AFRL data, the panels are used as uniform regions. The gray panel is removed from the analysis because it has visually identifiable spatial nonuniformity. Consequently, for each spectral band $k$, three points exist (one for each panel) through which a line is fit in order to estimate $a_{\hat{L}_{f}}$ and $b_{\hat{L}_{f}}$ using the slope and offset of the line respectively. Figure 3.17 displays the sample mean and sample variance estimated for each of the panels along with a linear fit through the

points. In comparison with the clusters of points in previous scatter plots such as Figures 3.10 and 3.13, the sample variance estimates associated with the panel regions 


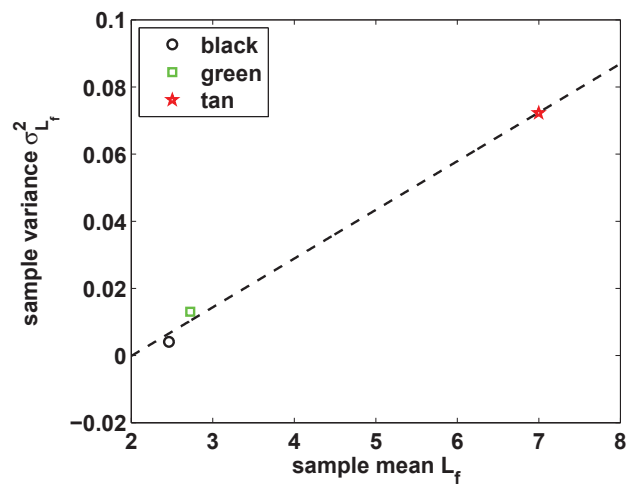

Figure 3.17: Sample mean versus sample variance estimated using all panel pixels for black, green, and tan panels for a single spectral band of AFRL hyperspectral data

are much larger, which results from spatial trends or nonuniformity over the entire panel region that is reduced when looking at smaller spatial regions on each panel. The gain and offset of the linear noise model are estimated for each band using this method and are shown in Figure 3.18. Overall, the estimates of the gain and offset

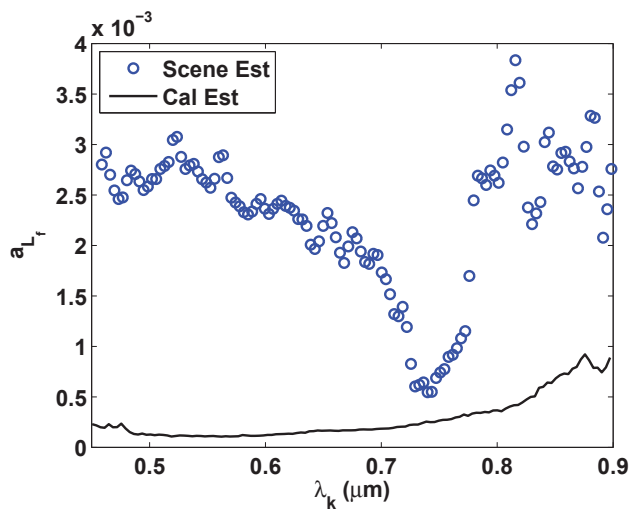

(a) Gain $\hat{a}_{\hat{L}_{f}}$

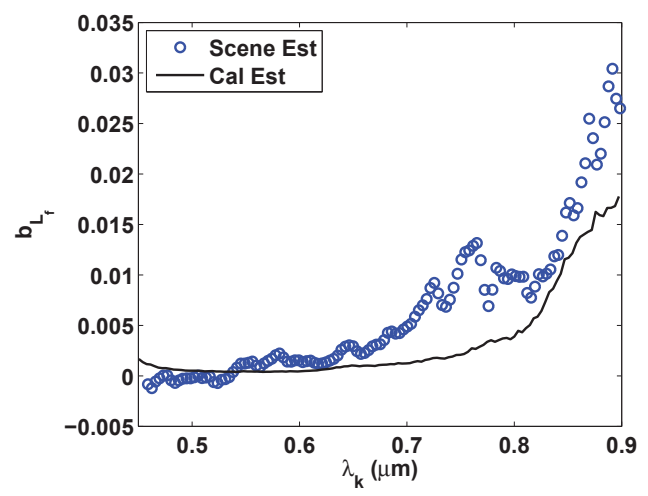

(b) Offset $\hat{b}_{\hat{L}_{f}}$

Figure 3.18: Comparison of noise model gain and offset estimates obtained using calibration data and in-scene methods 
deviate significantly from the estimates obtained using the calibration data. These differences have subsequently been attributed to spatial non-uniformity and trends across the panels, which are not identifiable upon visual inspection.

In Green's approach, each pixel is differenced with a neighboring pixel. The residual difference is then used to estimate the noise variance. This approach assumes that if a relatively uniform area exists within the scene, any spatial non-uniformity that exists will be very local and slowly varying. Consequently, differencing neighboring pixels should essentially remove these non-uniformities leaving residuals for which any variability is due explicitly to sensor noise. Due to the differencing, the estimated variance is twice that of the true noise variance, so the resulting estimate is reduced by one half. Green's original method assumes a signal-independent noise variance.

A modified version of Green's method is applied to the panels in the scene (excluding gray). The noise variance of the black, green, and tan panels are computed after differencing neighboring pixels. Linear regression is then performed to compute the gain and offset of the signal-dependent noise model. The sample variance is estimated using all of the panel pixels as done in the first case discussed. The corresponding sample mean is obtained for each panel using all the panel pixels as well. The gain and offset of the noise model are estimated by fitting a line through the sample mean and variance as in Figure 3.17. Essentially, the only difference between this approach and the results shown in Figure 3.17 is that the sample variance estimates are different due to the pixel differencing approach used. The estimated gain and offset for each spectral band are shown in Figure 3.19. The estimates have improved significantly when compared to those in Figure 3.18. However, the approach is very sensitive to the pixels manually identified as belonging to each panel, which are used to estimate 


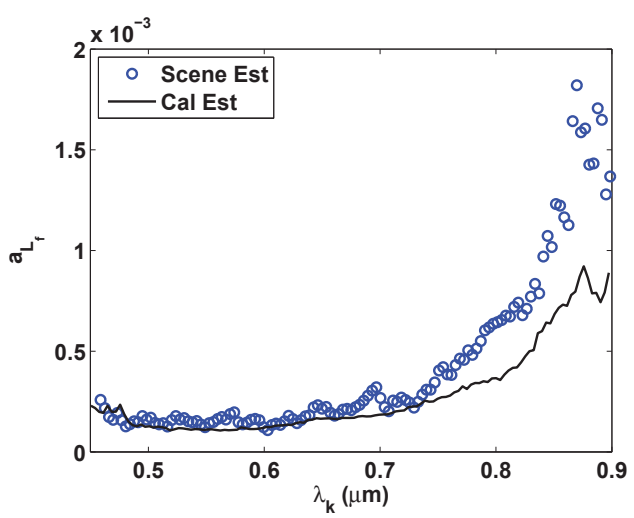

(a) Gain $\hat{a}_{L_{f}}$

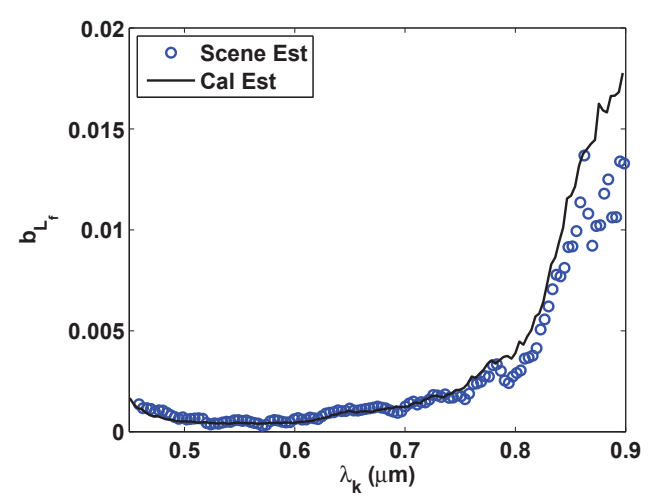

(b) Offset $\hat{b}_{L_{f}}$

Figure 3.19: Comparison of noise model gain and offset estimates obtained using calibration data and modified Green method

the sample variance. When the method was first applied to the panels in this experiment, the resulting gain and offset estimates were poor because pixels near the edge of the panels were used. Consequently, the user must be careful when selecting pixels in the scene to use for estimation.

Roger utilizes a multiple regression approach to remove local non-uniformity in the data. Essentially, he assumes that the measurement for a given pixel $(m, n)$ at spectral band $k$ can be modeled as a linear combination of the measurements at the same pixel at adjacent spectral bands $k-1$ and $k+1$ and the measurement of the neighboring pixel $(m-1, n)$ or $(m+1, n)$ at the same spectral band. He divides the scene into a number of blocks and applies and estimates the regression coefficients separately for each block. Any residual error present after regression results from sensor noise. Roger's method assumes a signal-independent noise. Consequently, a modified version of Roger's method is applied where the regression model is applied to produce a data set of residuals. These residuals are then used to estimate the 
noise variance of the panel regions in the scene from the same pixels used in Green's method above. The estimated noise variance along with the sample mean of each panel is used to fit a line to determine the noise model gain and offset. Figure 3.20 displays the estimated gain and offset using this method. The estimated noise variance

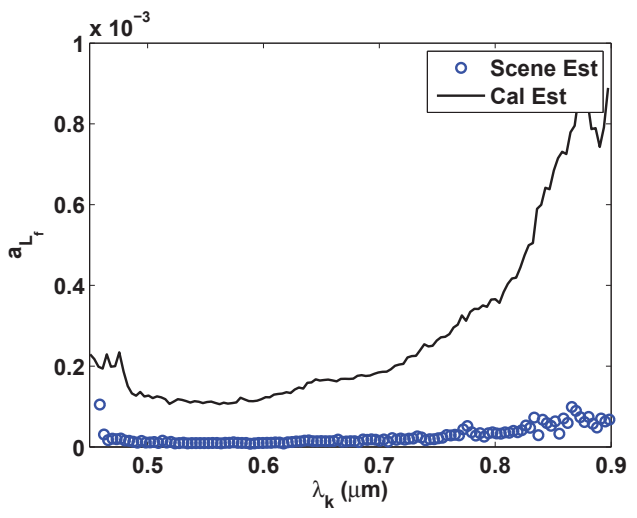

(a) Gain $\hat{a}_{L_{f}}$

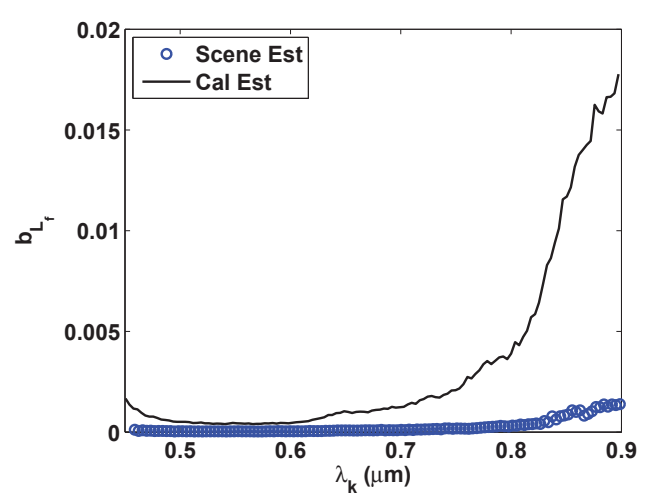

(b) Offset $\hat{b}_{L_{f}}$

Figure 3.20: Comparison of noise model gain and offset estimates obtained using calibration data and modified Roger method

using this method is much lower than the expected noise variance estimated from the calibration data. This may result from the regression method applied, which appears to reduce noise by projection onto a lower dimensional subspace. The performance of modified versions of Green's method and Roger's method have been compared with the estimates obtained from the calibration data. A direct comparison of the original form of both methods is difficult since both assume signal-independent noise. The noise estimated using these techniques is the overall combined signal-independent and signal-dependent noise. As a result, the noise estimates from these methods can only be compared with those from the calibration data for a particular signal 
level. In general, Green's baseline method tends to overestimate the noise variance whereas Roger's method tends to underestimate the noise variance. Overall, the approaches discussed here have limitations. In general, the approaches require the user to manually identify regions in the scene that are uniform, which may be difficult due to small local non-uniformity and illumination trends. Green's modified method tends to remove the local trend problems but again is sensitive to the pixels chosen as uniform areas. Roger's modified method tends to underestimate the noise variance due to projection onto a less noisy subspace. The supervised and unsupervised scatter plot methods provide alternative estimation approaches in cases where they can be applied.

\subsection{Significance of Noise Estimate for Change Detection}

The noise estimation discussion presented in this chapter has broad application in hyperspectral data exploitation. For the MB change detection approach, noise estimation is required to obtain an estimate of the noise covariance matrix $\boldsymbol{\Gamma}^{(t)}[m, n]$ used in the detection statistic of (2.23). In the development of the change detection method, the noise covariance is allowed to vary temporally $(t)$ and spatially $(m, n)$ to allow for signal-dependent noise. As discussed earlier, the noise is expected to be spectrally uncorrelated but colored. As a result, the noise covariance matrix is expected to be diagonal. The user can employ a linear function that describes the

diagonal elements of the noise covariance $\left(\sigma^{(t)}\right)^{2}\left[m, n, \lambda_{k}\right]$ as a function of signal level. From (3.12), the noise variance is estimated as

$$
\left(\sigma^{(t)}\right)^{2}\left[m, n, \lambda_{k}\right]=a_{L}\left[m, \lambda_{k}\right] L^{(t)}\left[m, n, \lambda_{k}\right]+b_{L}\left[m, \lambda_{k}\right]
$$


where $L^{(t)}$ is the measurement. Ideally, the noise estimate would be a function of the mean signal at time $t$ rather than the measurement $L$ itself. However, the MLE of the mean signal is the measurement due to a lack of further information.

For cases where limited noise estimates can be obtained, various dependencies for the noise covariance may be eliminated. As seen in the empirical noise estimation techniques discussed in this chapter, the spatial dependence of the gain $a_{L}$ and offset $b_{L}$ are often removed because this dependence cannot be estimated. In many cases, the noise statistics cannot be estimated from the imagery due to low spatial resolution of the sensor system. For airborne platforms, each spatial pixel may cover an area on the ground greater than 1 meter. As a result, finding spatially uniform areas within the scene can be difficult. In the cases where the noise cannot be estimated and no further a priori information about the noise exists, a whitened noise model must be used, i.e.,

$$
\boldsymbol{\Gamma}^{(t)}[m, n]=\boldsymbol{\Gamma}^{(t)}=\mathbf{I}
$$

where $\mathbf{I}$ is a $K \times K$ identity matrix. The significance of employing a signal-dependent noise covariance for the $\mathrm{MB}$ change detection method versus using a simpler noise model will be dependent on the data set itself. For data sets in which the signalindependent noise source dominates the system noise, the removal of the signaldependence will not be significant. Additionally, if the residual calibration error in the imagery is large, the impact on change detection using the model-based approach can potentially be greater than the lack of a signal-dependent noise model. Further testing on the use of a signal-dependent noise model is provided Chapter 4 . 


\section{Chapter 4: RESULTS}

\subsection{Introduction}

The performance of the model-based (MB) change detection technique is examined for various data sets. Included in the detection problem is the noise estimation problem discussed in Chapter 3. For each data set, the noise estimation performance and the impact on estimation/detection performance of changing various aspects of the model-based approach are discussed. The data sets tested here include an artificial scene generated using MODTRAN and laboratory reflectance signatures, the tower-based hyperspectral data used in the previous noise estimation chapter, airborne HYDICE data, and ARCHER data.

\subsection{MODTRAN Data}

The MB change detection approach is first tested on synthetic imagery, which has been generated using the assumed underlying radiative transfer model given in (2.2) where the RT terms have been generated using MODTRAN and the reflectance vectors have been taken from a spectral library. The use of synthetic imagery provides the advantage of having complete truth information about the RT conditions, shadow conditions, reflectance spectra of each pixel, and positions of the change targets within 
the scene. Consequently, the total performance, both estimation and detection, of the MB approach can be assessed.

\subsubsection{Data Summary}

An artificial scene of size $M=100$ and $N=100$ is constructed to represent a general rural scene with urban structure as well. The time-1 and time- 2 scenes are generated using radiative transfer data from MODTRAN for a wavelength range of $0.4-1.0 \mu m(400-1000 \mathrm{~nm})$ with $K=121$ spectral bands. More specifics about the operation of MODTRAN are given in Appendix A. The data are generated using the same haze model (rural), atmospheric model (mid-latitude summer atmosphere), and sensor height $(1 \mathrm{~km})$ for both time-1 and time-2. The time- 1 scene is generated with a solar zenith angle of $50^{\circ}, 25 \mathrm{~km}$ visibility, and a 0.8 scaling factor for the default water vapor column associated with the mid-latitude summer atmosphere model. For time-2, the solar zenith angle is switched to $25^{\circ}$, the visibility to $15 \mathrm{~km}$, and the default water vapor column is used without scaling. Figure 4.1 provides material map and reflectance information for the scene. The scene is generated using the following materials:

- Dry grass: light green

- Oak leaf: dark green

- Maple leaf: bright green

- Gray concrete: gray

- Silver paint: blue

- Terra cota roof tiles: red 
which are denoted by their indicated material map color.

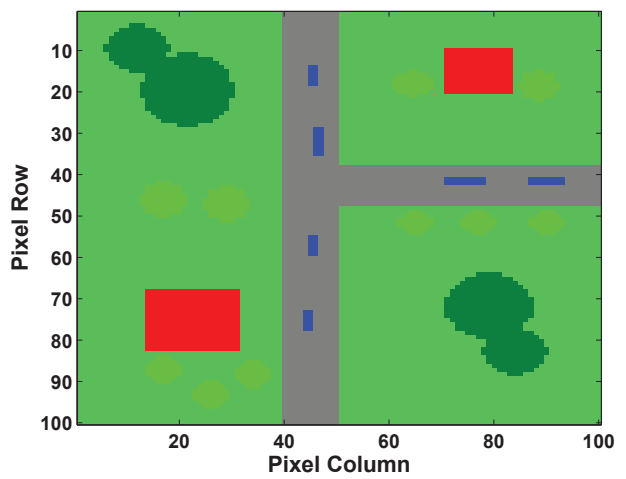

(a) Material map

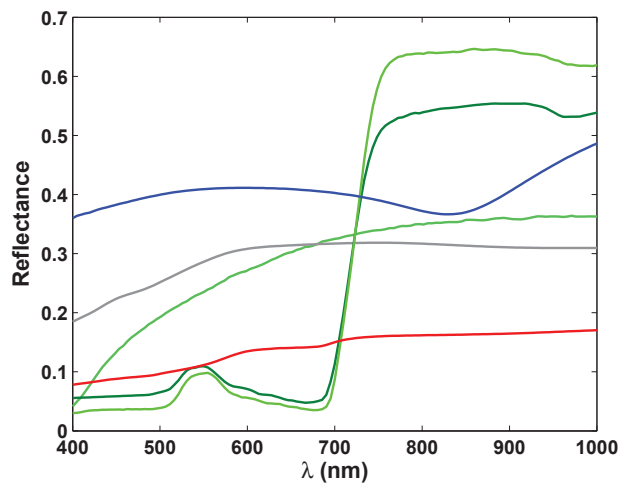

(b) Material reflectance spectra

Figure 4.1: Material reflectance truth data

Shadows are inserted to add the effects of shadow changes between observations for which the algorithm is aimed to ameliorate. The shadows are created randomly from a manually created binary shadow map of ones and zeros. If the shadow map for a given pixel is one, random shadow coefficients are applied for the pixel. If the shadow map is zero, the shadow coefficients are set to 1 . The direct solar shadow coefficients, $\alpha$ are generated as uniformly distributed random variables $\mathcal{U}(0,0.2)$ whereas the diffuse shadow coefficients are generated as $\mathcal{U}(0,0.6)$. Figure 4.2 and 4.3 display the true shadow coefficients $(\tilde{\alpha}, \beta)$ where the direct shadow coefficients are scaled by the cosine of the solar zenith angle. For all shadow coefficient images presented, white corresponds to $\tilde{\alpha}, \beta=1$ and black corresponds to $\tilde{\alpha}, \beta=0$.

A change target is inserted into the time-2 scene at various arbitrary locations. The targets are $2 \times 2$ pixels in size and correspond to a red-brick reflectance spectrum. Six different target locations are chosen resulting in a total of 24 target pixels $(0.24 \%$ 


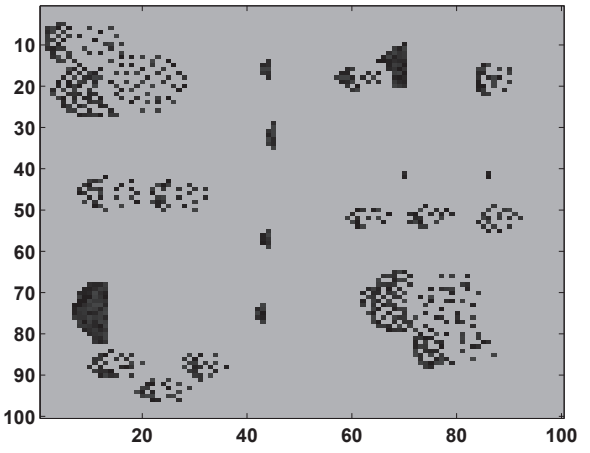

(a) $\tilde{\alpha}^{(1)}$

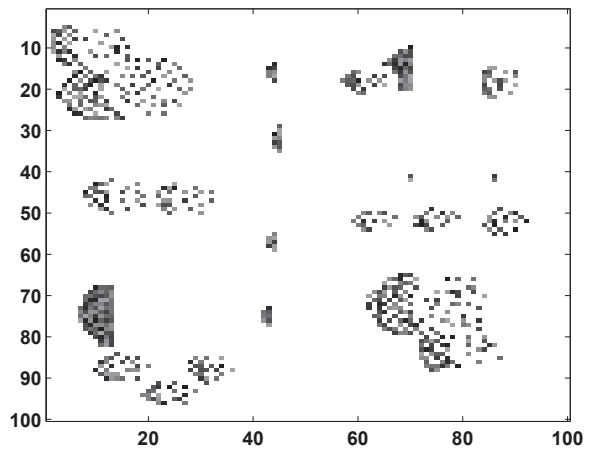

(b) $\beta^{(1)}$

Figure 4.2: Shadow coefficient truth for time-1 MODTRAN data

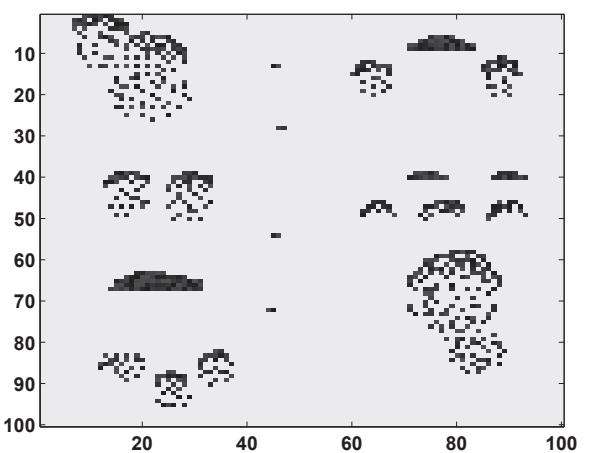

(a) $\tilde{\alpha}^{(2)}$

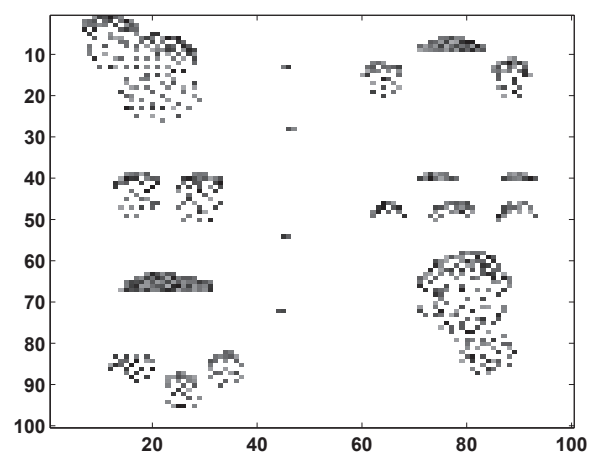

(b) $\beta^{(2)}$

Figure 4.3: Shadow coefficient truth for time-2 MODTRAN data 
of the pixels). The target truth map and reflectance signature are given in Figure 4.4 .

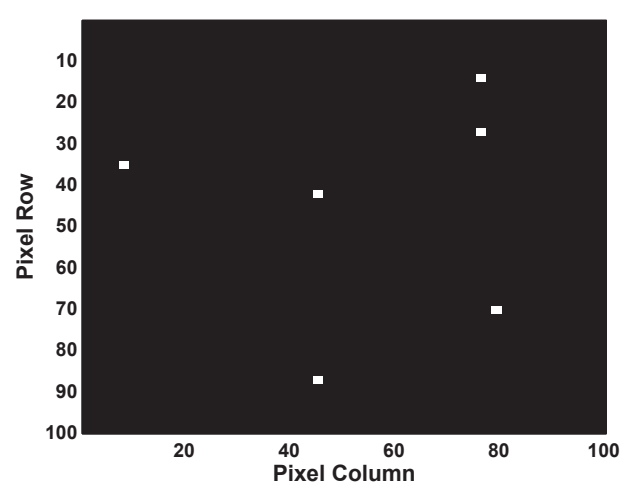

(a) Target map

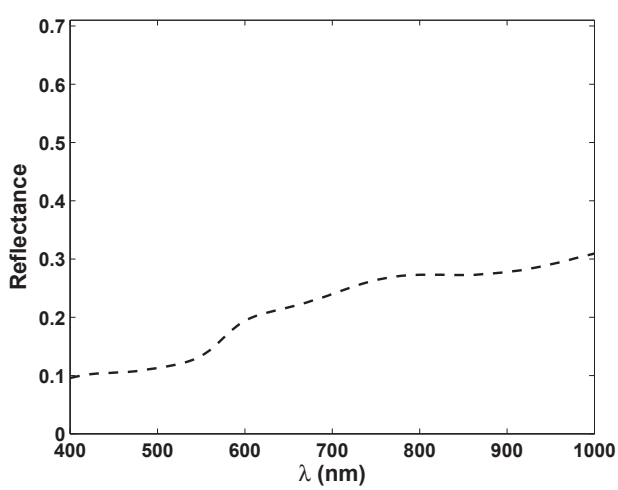

(b) Target reflectance spectrum

Figure 4.4: Target truth for MODTRAN data

A signal-dependent sensor noise model as discussed in Chapter 3 is used for which the variance is given by

$$
\sigma^{2}\left[m, n, \lambda_{k}\right]=a_{L} L\left[m, n, \lambda_{k}\right]+b_{L}
$$

with $a_{L}=3 \times 10^{-3}$ and $b_{L}=0.05$. The simulated noise characteristics are similar to those of the AFRL data examined in the previous chapter. Figure 4.5 displays color images for the simulated time-1 and time-2 data generated using red, green, and blue bands from the hyperspectral data cube. Most of the change targets inserted into the time-2 scene are fairly apparent except for one present in the upper right quadrant of the scene in the terra cota material class. These two material reflectance spectra are similar, making the change less apparent. 


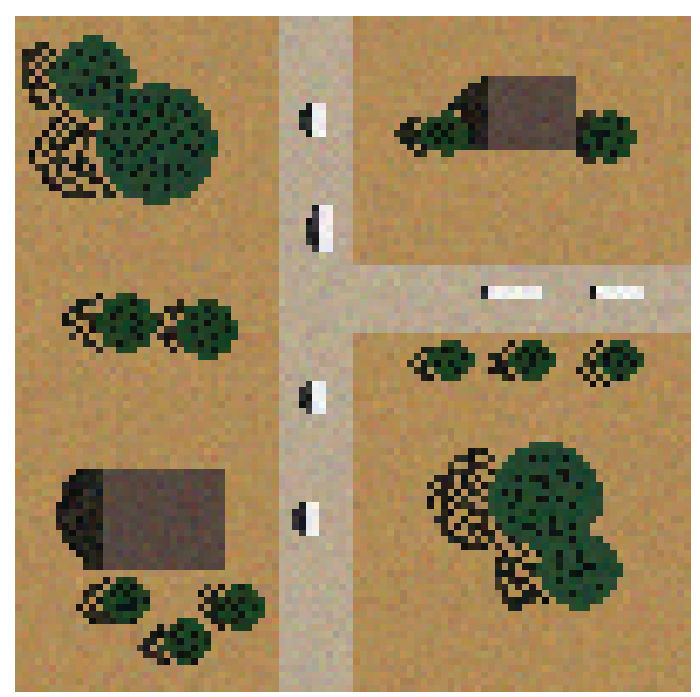

(a) Time-1 data

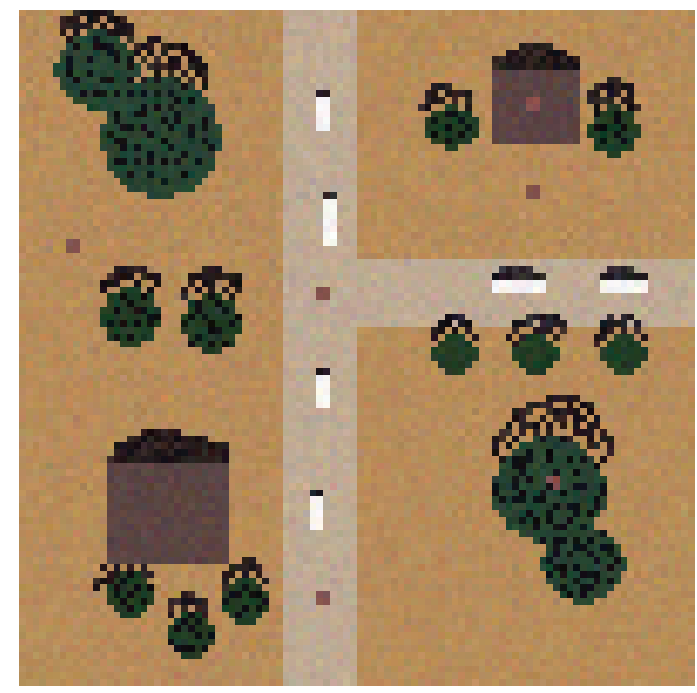

(b) Time-2 data with targets inserted

Figure 4.5: Color images of simulated noisy MODTRAN data

\subsubsection{Noise Estimation}

While the noise characteristics of the data are known in this case a priori, the unsupervised noise estimation technique described in Chapter 3 is applied to the data for comparison. The unsupervised method is implemented using a square window of size $9 \times 9$ with 40 classes used for mean class segmentation. Figure 4.6 displays the estimates in comparison with the spectrally constant known values used when generating the data. While a degree of estimation error does exist, the estimates tend to fluctuate around the true values. The spectral mean of the estimated gain and offset are $4.8 \times 10^{-3}$ and 0.0446 , which are relatively close to the true values of $3 \times 10^{-3}$ and 0.05 . For the remaining simulations, the true gain and offset are used to assess change detection performance in the absence of noise estimation error. 


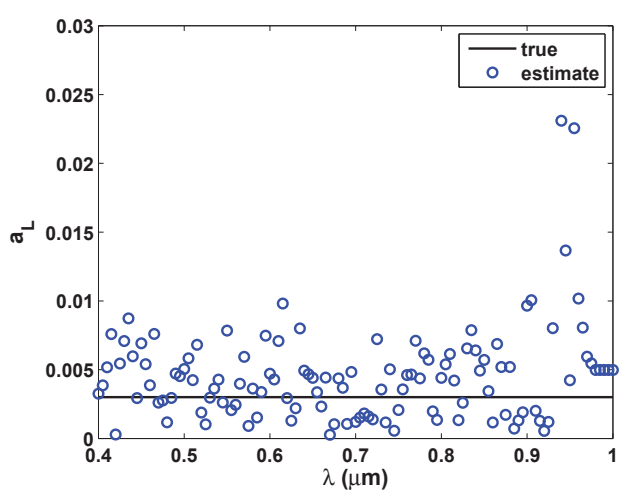

(a) Gain $\hat{a}_{L}$

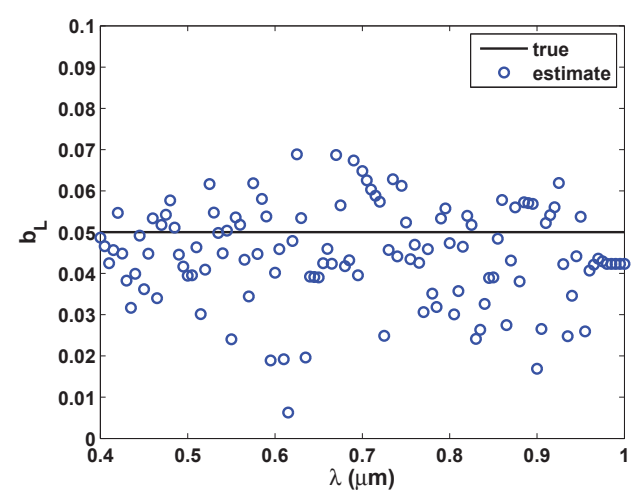

(b) Offset $\hat{b}_{L}$

Figure 4.6: Estimated noise model gain and offset obtained using unsupervised scatter plot method for MODTRAN data

\subsubsection{Results}

The MB algorithm is applied to this data set for a number of different configurations and initializations. For these simulations, an RT subspace dimensionality of $I=10$ is used based upon subspace error and bias error examination in previous work [53] and based upon detection performance examination as a function of $I$ for which results are shown later in this section. The RT conditions are initialized assuming the sensor altitude and solar position are known, which is a fair assumption. MODTRAN is used to generate RT conditions. using the standard mid-latitude summer atmospheric profile with an average visibility of $20 \mathrm{~km}$ for both time- 1 and time- 2 and using the default water vapor column associated with the atmosphere model. This initial assumption differs from that used to simulate the data. Figure 4.7 displays the resulting initial estimates of the RT terms in comparison with the true terms used when generating the data. From a physical standpoint, the true visibility of the time-1 data is greater than that of the initial estimate and the water vapor content 


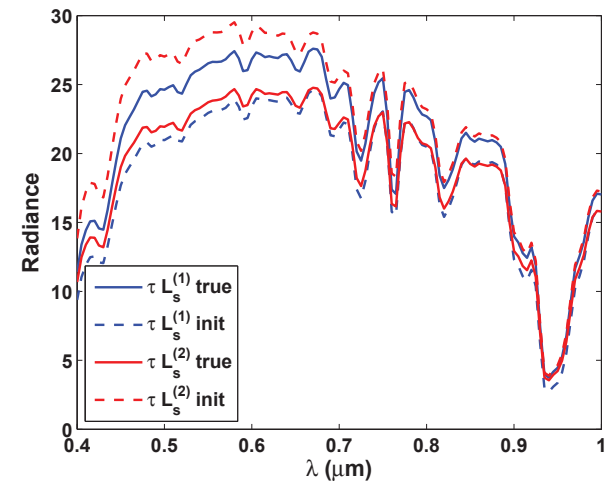

(a) $\boldsymbol{\tau} \odot \mathbf{L}_{s}$

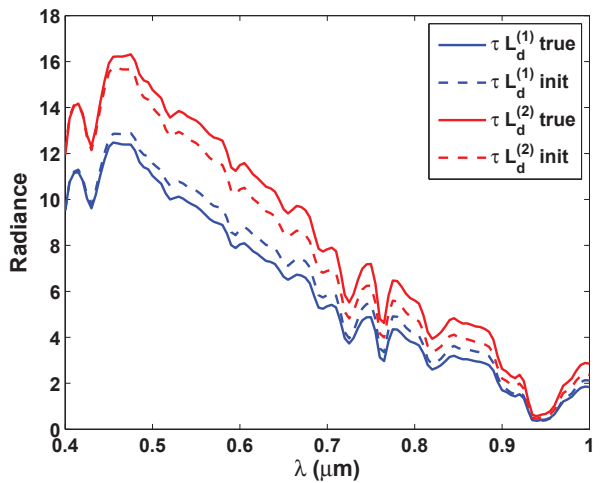

(b) $\boldsymbol{\tau} \odot \mathbf{L}_{d}$

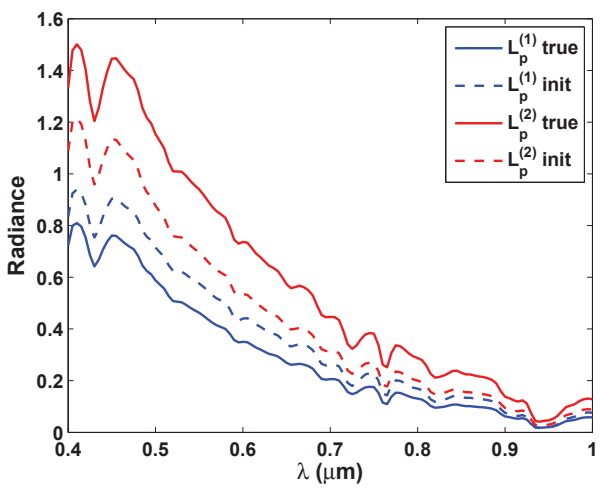

(c) $\mathbf{L}_{p}$

Figure 4.7: Initial estimates of RT terms for MODTRAN data compared with truth 
is lower, which ultimately results in higher atmospheric transmission. As a result, the true direct solar radiance $\boldsymbol{\tau} \odot \mathbf{L}_{s}$ is greater than the initial estimate. Similarly, the true diffuse radiance $\boldsymbol{\tau} \odot \mathbf{L}_{d}$ and path radiance $\mathbf{L}_{p}$ are less than the initial estimates. Additionally, the assumed visibility for the time-2 data is greater than the true visibility resulting in a higher direct solar radiance and lower diffuse and path radiance.

The estimation and detection results are compared for the MB algorithm run in the three different reflectance estimation modalities: 1) no spectral filtering of reflectance (NF), 2) spectral filtering of reflectance (SF), and 3) reflectance subspace representation using Legendre basis functions (RS). For the spectral filtering implementations of the algorithm, a Bartlett window of length 7 is used. Similarly, a reflectance subspace dimension of $I_{r}=40$ is used when implementing the Legendre subspace representation, as discussed earlier. Algorithm performance is assessed for several different initializations of the shadow coefficients. In particular, the percentage of pixels classified as "shadow pixels" for initialization is varied. Additionally, the algorithm is tested using perfect initial estimates of both the shadow coefficients and RT basis coefficients, which will serve as a baseline for change detection performance. For shadow initialization, pixels identified as shadow pixels are initialized with a direct shadow coefficient value of $\alpha=0.1$ and a diffuse shadow coefficient value of $\beta=0.5$. The remaining non-shadow pixels are initialized with $\alpha=1$ and $\beta=1$. The geometric cosine projection term is initialized using the known solar zenith angle as $\cos \theta_{m, n}^{(t)}=\cos \theta_{s}^{(t)}$. For all the simulations, 10 iterations of the $\mathrm{AO}$ algorithm is used. Figure 4.8 displays the convergence criteria given in (2.56) and (2.57) for one particular case for the MODTRAN data. Both of the convergence criteria decrease in 


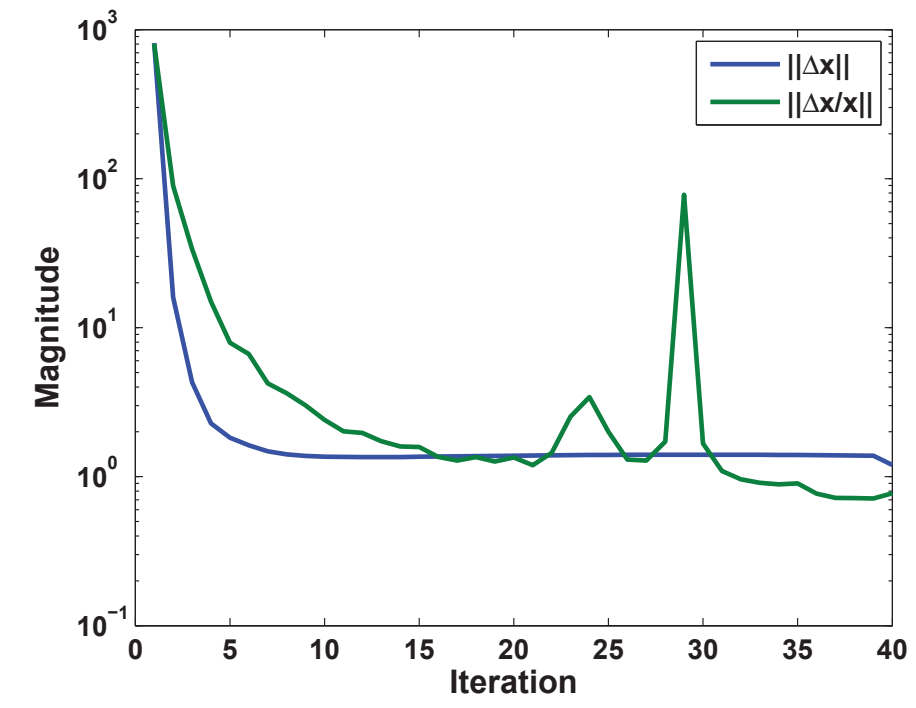

Figure 4.8: Convergence criteria as a function of iteration for AFRL data

general. The normalized convergence criteria demonstrates discontinuous behavior. No appreciable changes in detection performance or state estimates is observed for iterations beyond 10. As a result, 10 iterations are applied for testing of MODTRAN data.

\section{$5 \%$ Shadow Initialization}

The histogram threshold method discussed in Section 2.4.3 is used to detect the bottom $5 \%$ of spectral norm pixels in both the time- 1 and time- 2 scenes. These pixels are detected as shadow pixels and initialized with the shadow coefficient values given above. Figures 4.9 and 4.10 display the initial shadow coefficient estimates for the time-1 and time-2 data respectively. In comparison with the shadow truth given in Figures 4.2 and 4.3, the shadow initialization is relatively accurate. However, a portion of shadow pixels are not detected because the true number of shadow pixels 


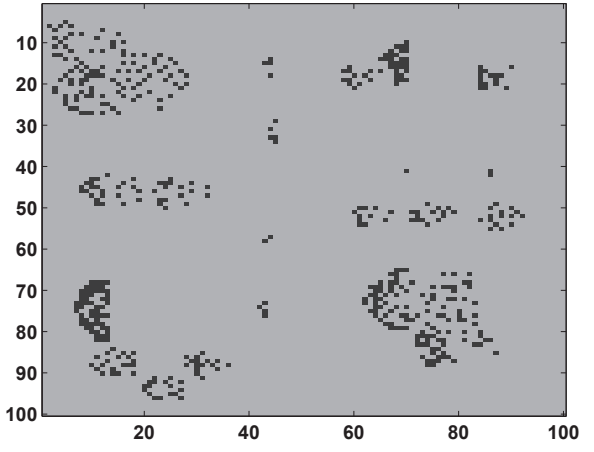

(a) $\tilde{\alpha}^{(1)}$

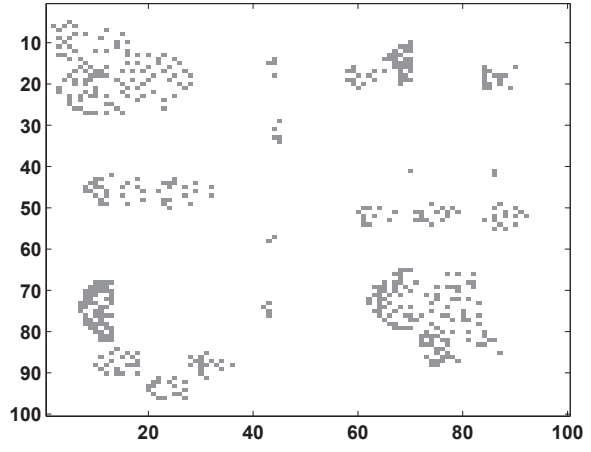

(b) $\beta^{(1)}$

Figure 4.9: Initial estimates for time-1 shadow coefficients using bottom $5 \%$ of spectral norm pixels for MODTRAN data

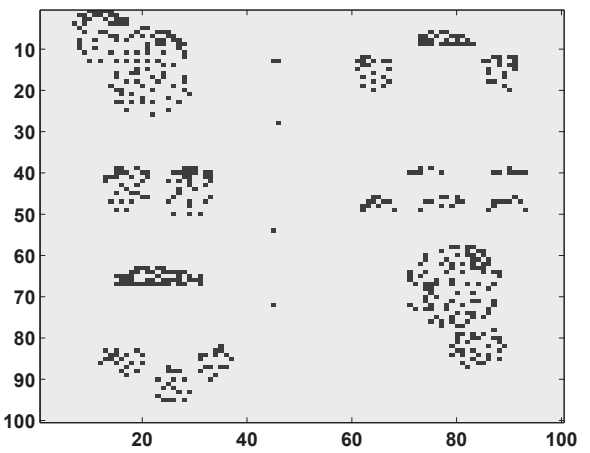

(a) $\tilde{\alpha}^{(2)}$

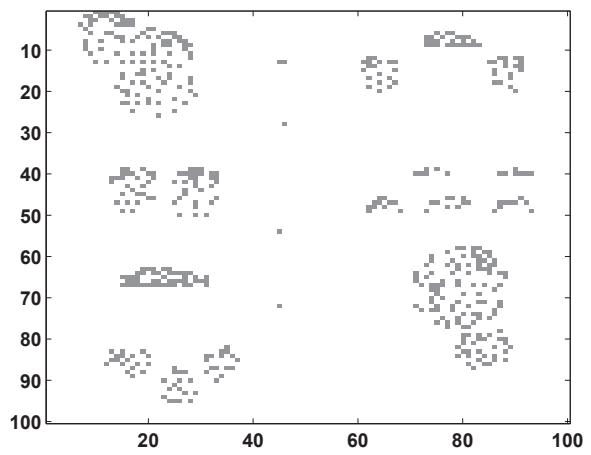

(b) $\beta^{(2)}$

Figure 4.10: Initial estimates for time-2 shadow coefficients using bottom $5 \%$ of spectral norm pixels for MODTRAN data 
is closer to $7 \%$. Additionally, the initial estimates of the coefficients for shadow pixels do not match exactly due to the random nature in which the true coefficients were generated.

The resulting model parameter estimates using this initialization are obtained and compared with their true values. Figure 4.11 displays the RT estimates for time-1 for the three different reflectance estimation modalities. Figure 4.12 displays the

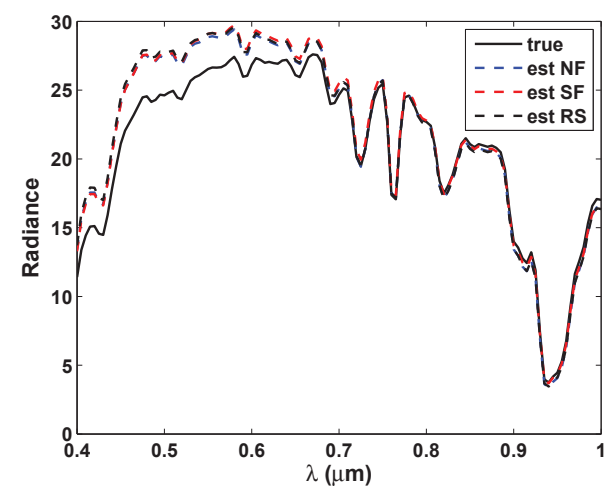

(a) $\boldsymbol{\tau} \odot \mathbf{L}_{s}$

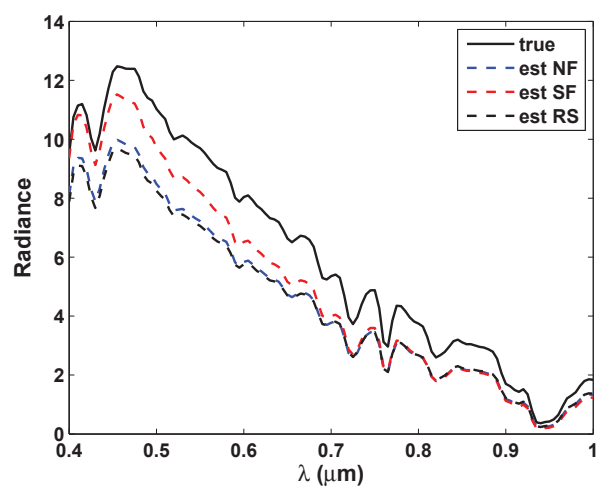

(b) $\boldsymbol{\tau} \odot \mathbf{L}_{d}$

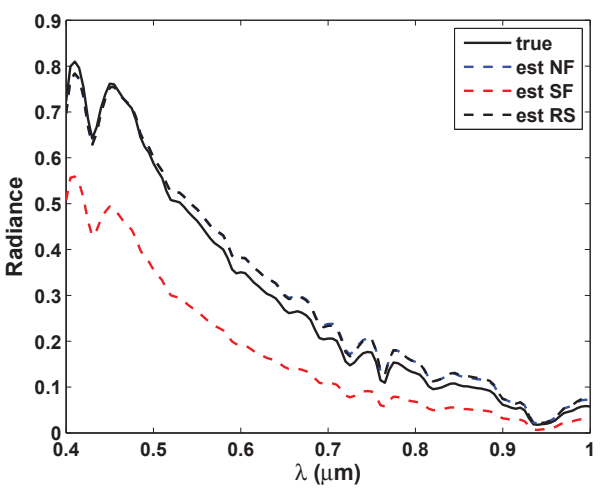

(c) $\mathbf{L}_{p}$

Figure 4.11: RT estimates for time-1 MODTRAN data for different reflectance estimation modalities using $5 \%$ shadow initialization.

illumination estimates for time-2. In general, the direct solar radiance term is overes- 


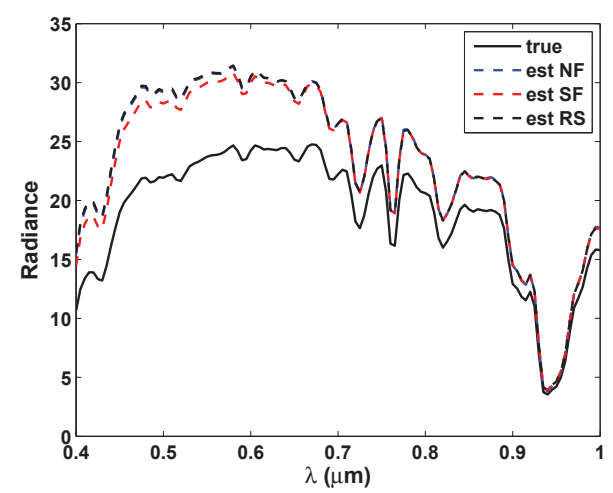

(a) $\boldsymbol{\tau} \odot \mathbf{L}_{s}$

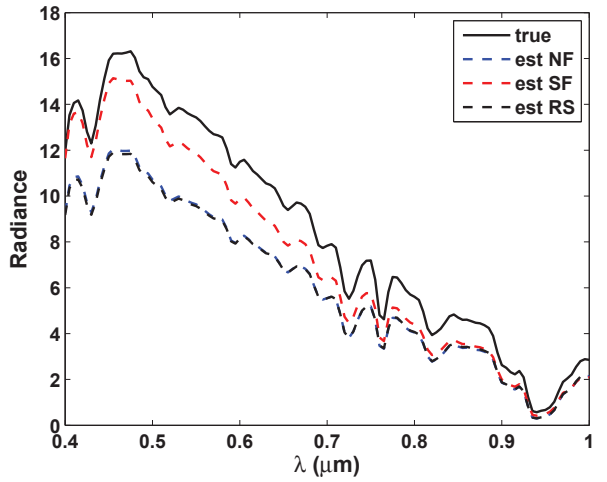

(b) $\boldsymbol{\tau} \odot \mathbf{L}_{d}$

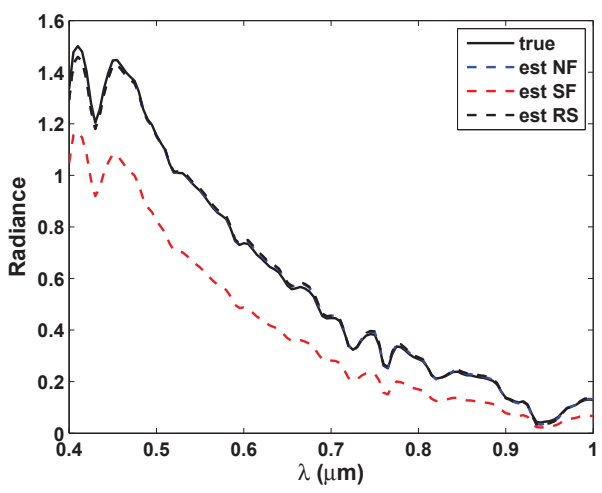

(c) $\mathbf{L}_{p}$

Figure 4.12: RT estimates for time-2 MODTRAN data for different reflectance estimation modalities using $5 \%$ shadow initialization. 
timated for both the time- 1 and time- 2 data, whereas the diffuse and path radiance terms are underestimated. This results from the combined inaccurate RT initialization and shadow initialization. Despite the inaccuracy of the final RT estimates, the residual error for non-shadow pixels is still relatively small. Figure 4.13 displays the predicted measurement $\hat{L}$ for a dry grass pixel for both the time- 1 and time- 2 data. Figure 4.14 displays the same for an oak leaf pixel. The residual measurement error

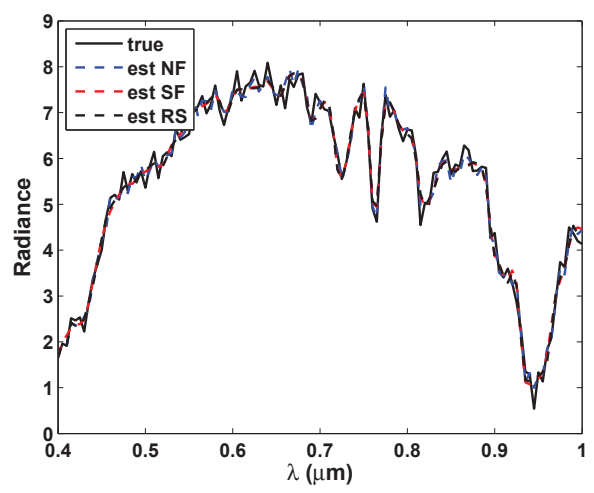

(a) Time-1

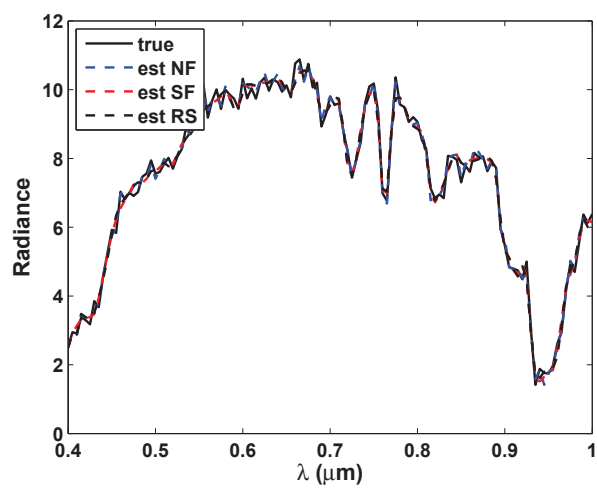

(b) Time-2

Figure 4.13: Comparison of predicted measurement $\hat{L}$ with true measurement for dry grass pixel using $5 \%$ shadow initialization for MODTRAN data

for these pixels results mostly from noise in the measurements. Additionally, the reflectance estimates for the materials associated with these pixels are nearly perfect as demonstrated in Figure 4.15. The spectral filtering and reflectance subspace methods produce smoother, more accurate reflectance measurements.

Figures 4.16 and 4.17 display the estimated shadow coefficients $\tilde{\alpha}$ and $\beta$ for the time-1 and time-2 data respectively. The shadow estimates using the various reflectance estimation modalities are all very similar. Consequently, only the results for 


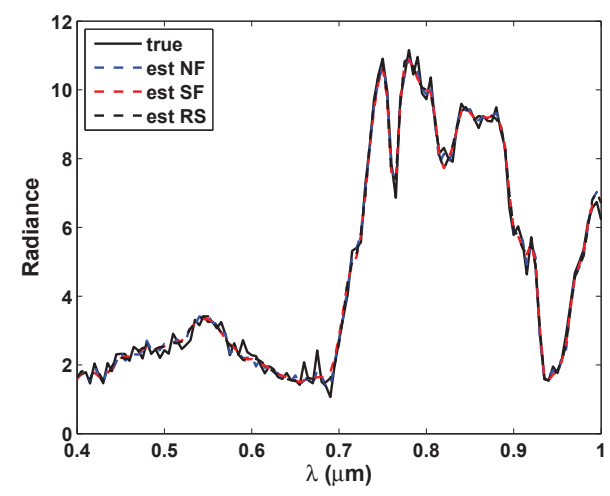

(a) Time-1

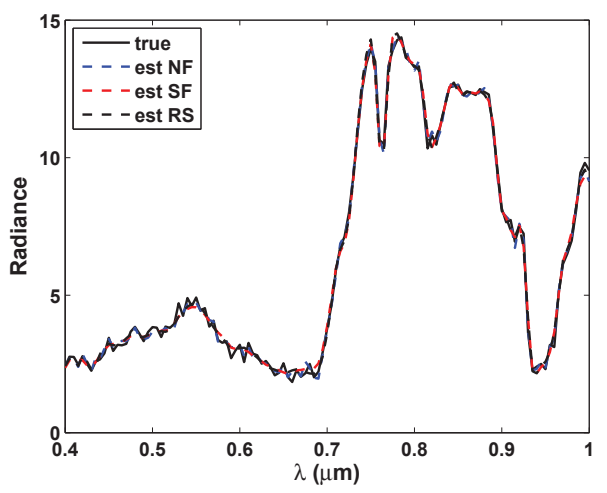

(b) Time-2

Figure 4.14: Comparison of predicted measurement $\hat{L}$ with true measurement for oak leaf pixel using $5 \%$ shadow initialization for MODTRAN data

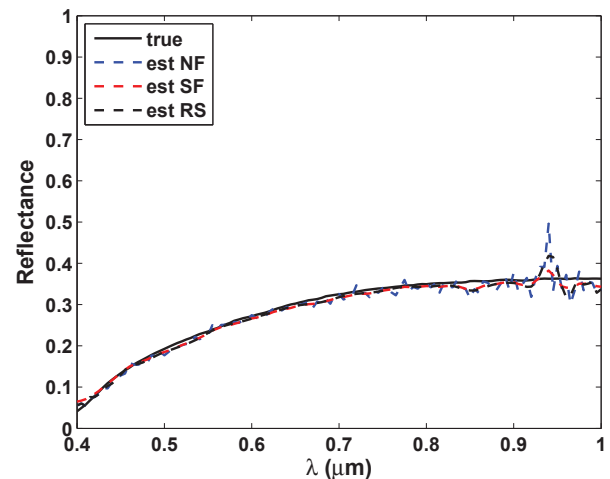

(a) Dry grass

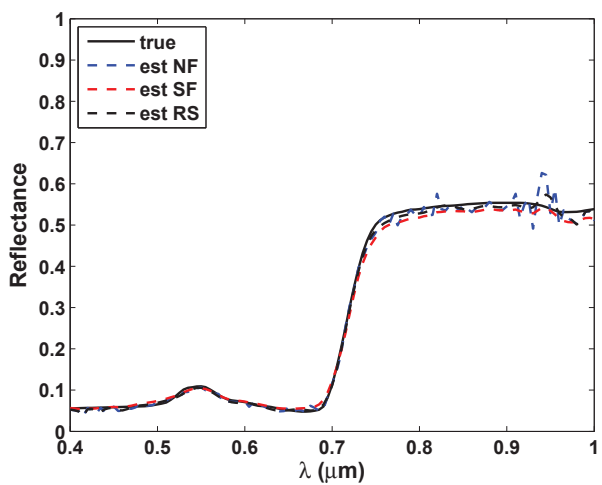

(b) Oak leaf

Figure 4.15: Comparison of estimated reflectance $\rho$ with true reflectance for grass pixel and oak leaf pixel using $5 \%$ shadow initialization for MODTRAN data 
the baseline no spectral filtering method are shown. Several interesting observations

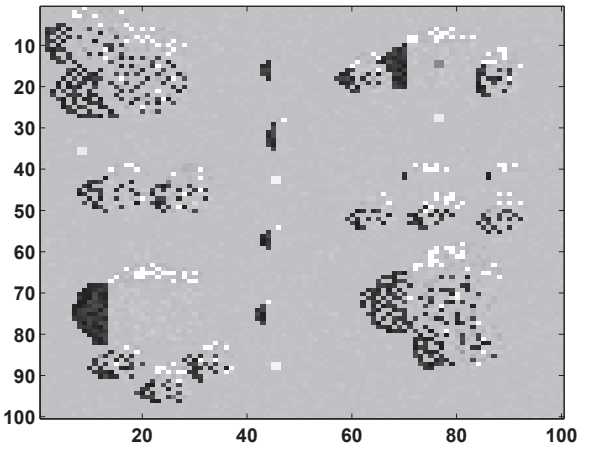

(a) $\hat{\tilde{\alpha}}^{(1)}$

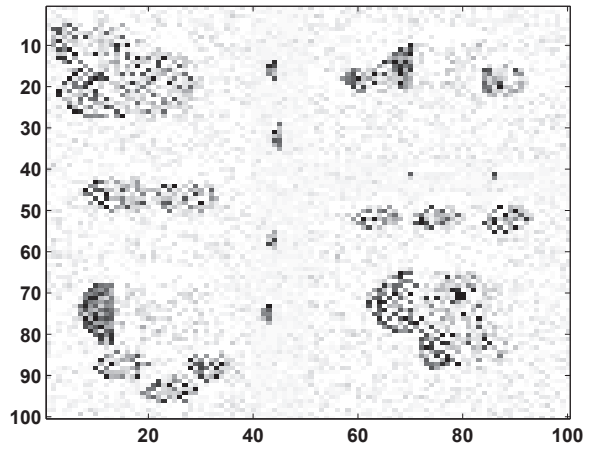

(b) $\hat{\beta}^{(1)}$

Figure 4.16: Estimated shadow coefficients for time-1 using 5\% shadow initialization for MODTRAN data

can be made concerning the shadow estimates. To begin, the mean of the direct shadow coefficient estimates $\hat{\tilde{\alpha}}^{(t)}$ should approximately equal $\cos \theta_{s}^{(t)}$ for non-shadow pixels. For this simulated data, $\theta_{s}^{(1)}=50^{\circ}$ with $\cos 50^{\circ}=0.6428$ and $\theta_{s}^{(2)}=25^{\circ}$ with $\cos 25^{\circ}=0.9063$. The sample mean of the time- 1 and time- 2 direct shadow coefficients are 0.7145 and 0.8879 respectively, which are greater than and less than the respective true values. Additionally, the mean of the indirect shadow coefficient estimates $\hat{\beta}^{(t)}$ should approximately equal 1 for non-shadow pixels. The sample means of the time- 1 and time-2 indirect shadow coefficients are 0.9421 and 0.9415 . The estimation error results, in large part, from the inaccurate initial estimates of the RT conditions. These results demonstrate that the algorithm can produce a combination of inaccurate shadow estimates and inaccurate RT estimates that still provide a relatively accurate description of the total downwelling radiance, i.e. $\tilde{\alpha} L_{s}+\beta L_{d}$, for 


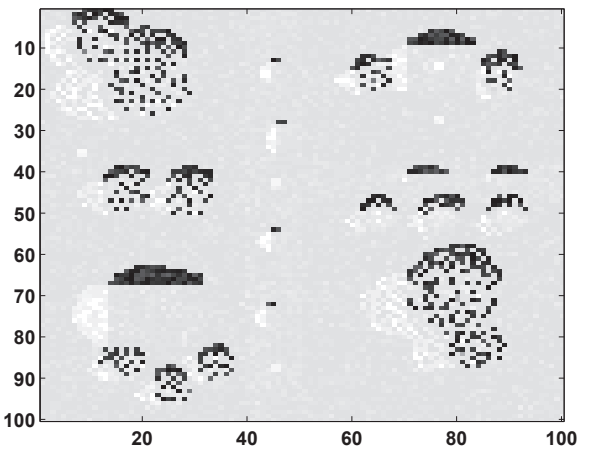

(a) $\hat{\tilde{\alpha}}^{(2)}$

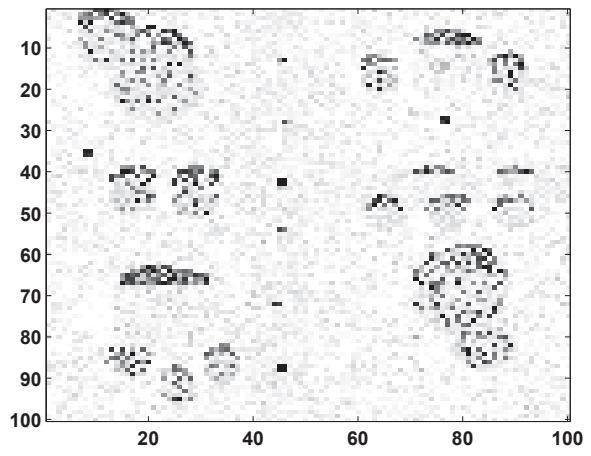

(b) $\hat{\beta}^{(2)}$

Figure 4.17: Estimated shadow coefficients for time-2 using 5\% shadow initialization for MODTRAN data

the majority of non-shadow scene pixels. One set of estimates will compensate for the other. Figure 4.18 displays the estimated total downwelling radiance at time-1 and time-2 in comparison with the true downwelling radiance to demonstrate how the combined inaccurate estimates of the RT terms and shadow coefficients compensate each other to produce an accurate downwelling estimate. Additionally, the inaccurate shadow initialization manifests itself mainly in the direct shadow coefficient estimates for pixels in shadow. Those shadow pixels in one scene that were not detected initially appear as bright pixels in the direct shadow coefficient estimates of the same pixels in the other scene as demonstrated in Figures 4.16 and 4.17. This results from the reflectance estimates after the first step of the AO algorithm. Since the pixels are not initially identified as shadow pixels, the reflectance estimates associated with those pixels are underestimated to compensate for the low measurement. An example of this underestimate is shown in Figure 4.19. On the subsequent shadow estimation step of the AO algorithm, the shadow coefficient of the associated pixel is 


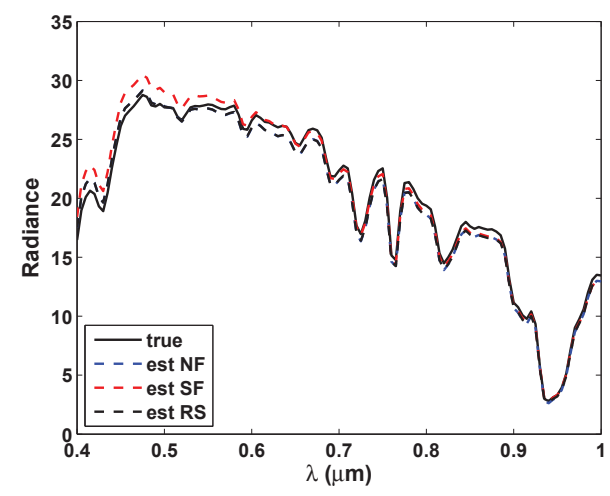

(a) Time-1

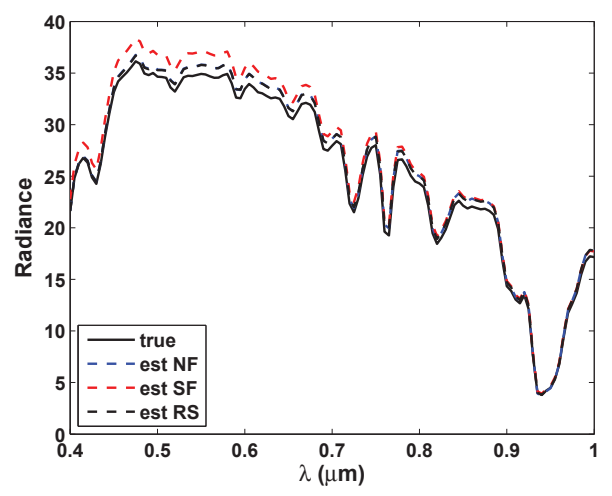

(b) Time-2

Figure 4.18: Estimated total downwelling radiance $\tilde{\alpha} L_{s}+\beta L_{d}$ for non-shadowed pixels using $5 \%$ shadow initialization in comparison with truth for MODTRAN data

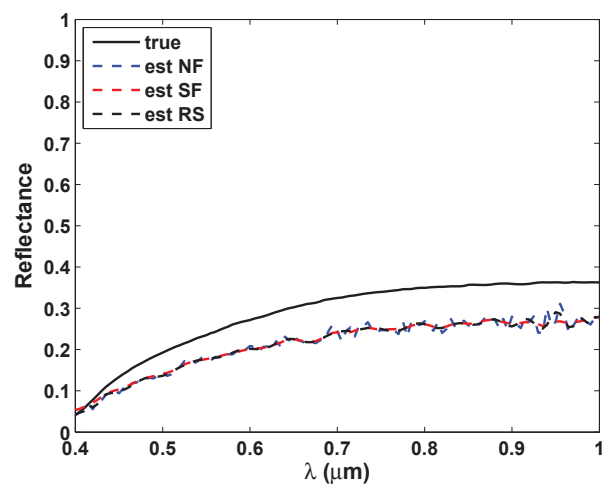

Figure 4.19: Underestimated reflectance for dry grass pixel due to poor shadow initialization for MODTRAN data 
overestimated for the scene in which the pixel is not in shadow to compensate for the underestimated reflectance.

The issue of prime importance, however, is not the parameter estimation accuracy, but how inaccuracies in the parameter estimates for the $\mathrm{AO}$ algorithm affect detection performance. The distribution of the detection statistic $\tilde{D}_{G}$ given in (2.16) is used to examine this further. As discussed, the detection statistic should be distributed as $\tilde{D}_{G} \sim \chi^{2}(2 K)$, where in this case $K=121$. Additionally, a mean of $2 K=242$ and a variance of $4 K=484$ are expected. Figure 4.20 displays the estimated distribution of the detection statistic for the three reflectance estimation modes in comparison with the theoretical distribution. The expected value and variance of the detection

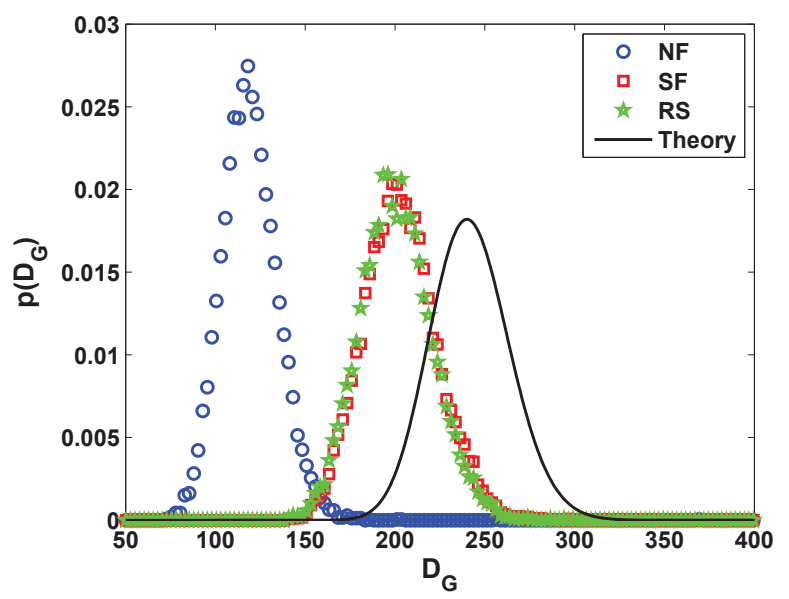

Figure 4.20: Sample distribution of detection statistic for different reflectance estimation modalities using $5 \%$ shadow initialization in comparison with theoretical distribution for MODTRAN data

statistic without reflectance filtering are 119.1 and 236.8, which are much less than the theoretical expected values. This results in part from the degrees of freedom 
in the reflectance estimation that essentially fit part of the random noise. These added degrees of freedom reduce the optimization error. The reflectance filtering and subspace representation both produce detector outputs distributed much closer to the theoretical distribution since the degrees of freedom are further constrained. However, the sample mean and variance of both are still less than expected. The distribution of the detector output provides an indicator of estimation performance, but the impact on detection performance may not be straightforward. Figure 4.21 displays the pixel-based empirical ROC curve performance of the MB algorithm using the three reflectance estimation modalities in comparison with the performance of the baseline $\mathrm{CC}$ and $\mathrm{CE}$ change detection algorithms discussed in the introductory material. In general, all the algorithms perform well by identifying greater than $60 \%$ of

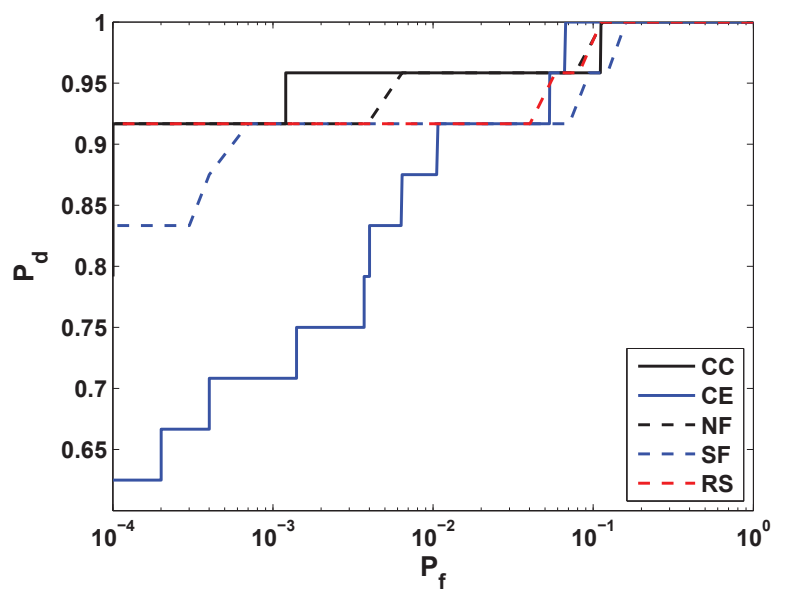

Figure 4.21: Empirical ROC curve performance for different reflectance estimation modalities using $5 \%$ shadow initialization in comparison with $\mathrm{CC}$ and $\mathrm{CE}$ algorithms for MODTRAN data

the target pixels with no false alarms. The performance of the the MB algorithms are 
similar except for a slight drop in performance for the SF method at low false alarms. Examination of the detection statistic images provides further insight into clutter suppression performance, sources of false alarms, and missed detections. Figure 4.22 displays the detection statistic images of the $\mathrm{CC}$ and $\mathrm{CE}$ algorithms respectively. Figure 4.23 displays the detection statistic images for the three reflectance modalities

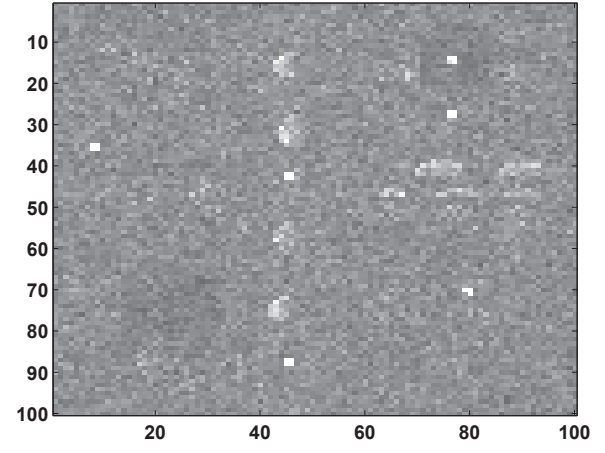

(a) $\mathrm{CC}$

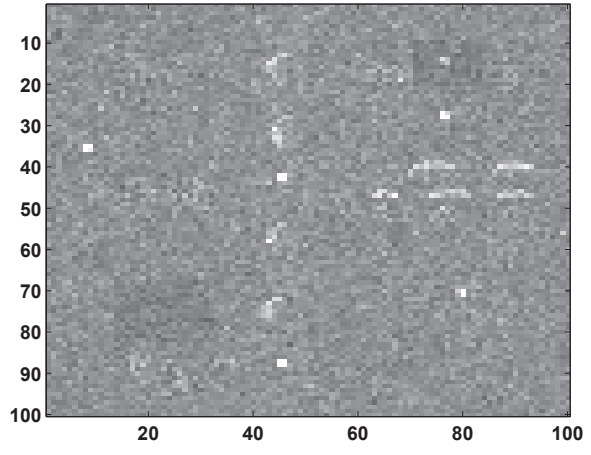

(b) $\mathrm{CE}$

Figure 4.22: Comparison of detection statistic images for CC and CE change detection algorithms for MODDTRAN data

of the MB method. The dynamic range of the images is stretched between 0 and $\mu+7 \sigma$ where $\mu$ is the sample mean of the detection statistic and $\sigma$ is the sample standard deviation. Essentially, all detector outputs greater than $\mu+7 \sigma$ are set to this value to accentuate more subtle detections and false alarms closer to the background distribution. The detection statistic images for the $\mathrm{CC}$ and $\mathrm{CE}$ display a much higher degree of residual clutter than the MB approaches. The majority of the residual clutter exists in the shadow regions of the scenes, which the MB approaches are specifically designed to improve. Indeed, the detection statistic images associated with 


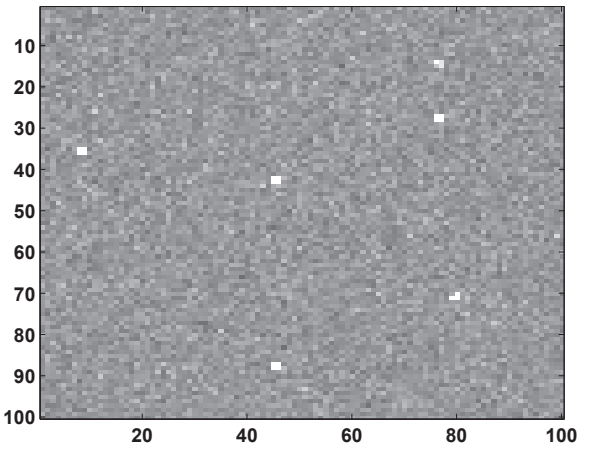

(a) No reflectance filtering

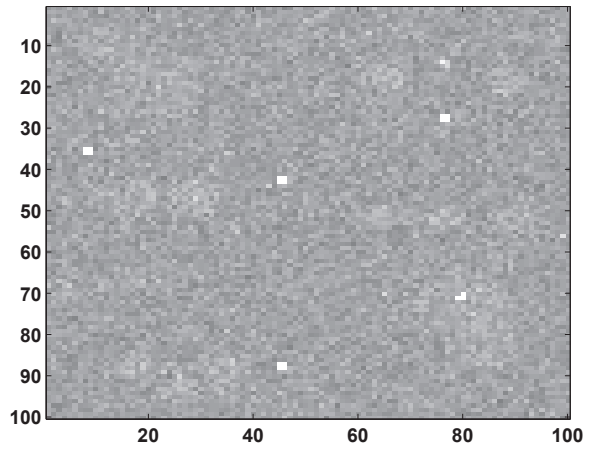

(b) Reflectance filtering

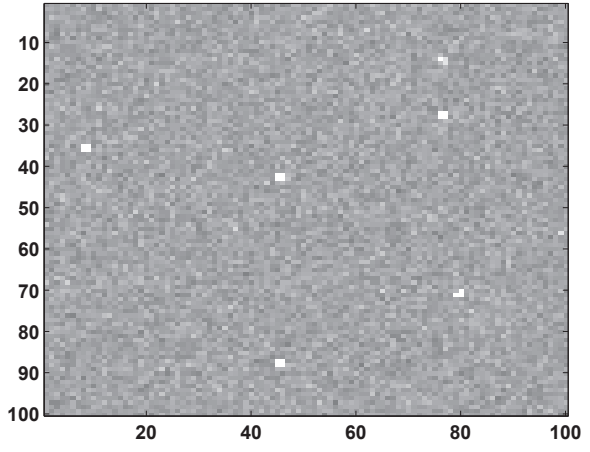

(c) Reflectance subspace

Figure 4.23: Comparison of detection statistic images for different MB reflectance modalities using $5 \%$ shadow initialization for MODTRAN data 
the MB approaches demonstrate very little residual clutter and resemble random noise with bright peaks for the detected change targets. However, the $\mathrm{CC}$ algorithm still performs well because the change target signal is larger than the residual background clutter in the scene. Using an appropriate threshold, the MB approaches identify the majority of change targets with no false alarms. The single exception is the change target positioned on the terra cota roof location. The change in reflectance from terra cota to red brick is subtle and neither possess strong spectral features as shown in Figure 4.24. As a result, the MB algorithm has difficulty detecting this change target

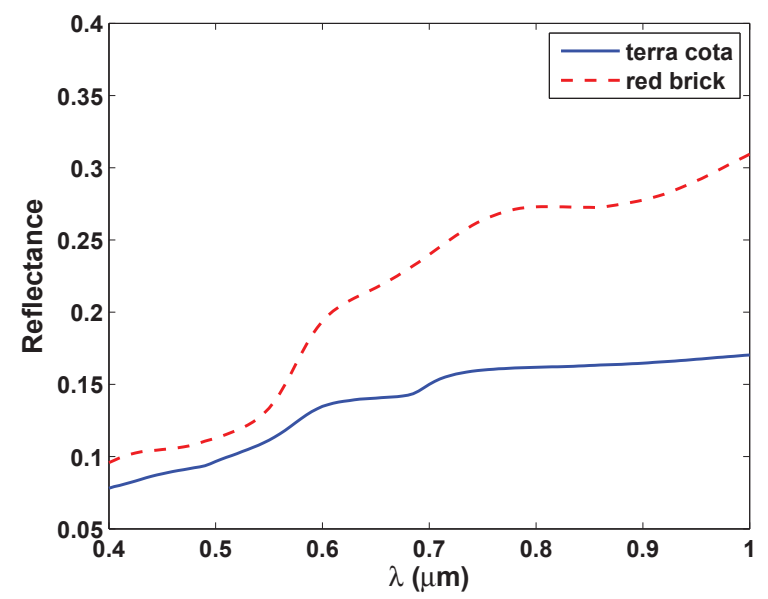

Figure 4.24: Comparison of reflectance spectrum for terra cota material and red brick material used in MODTRAN data

at lower false alarm rates. Overall, no definitive conclusions can be drawn from this single data set. However, it does serve as a proof of concept for application of the MB algorithm. 


\section{0\% Shadow Initialization}

Next, the impact of a different shadow initialization on estimation and detection performance is examined. The histogram threshold method is now used to detect the bottom $10 \%$ of spectral norm pixels in both the time- 1 and time- 2 scenes for shadow initialization. Figures 4.25 and 4.26 display the initial shadow coefficient estimates for the time-1 and time-2 data respectively. In comparison with the shadow truth given

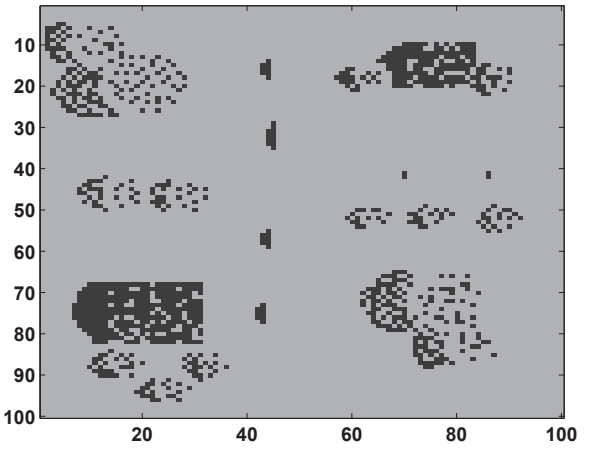

(a) $\tilde{\alpha}^{(1)}$

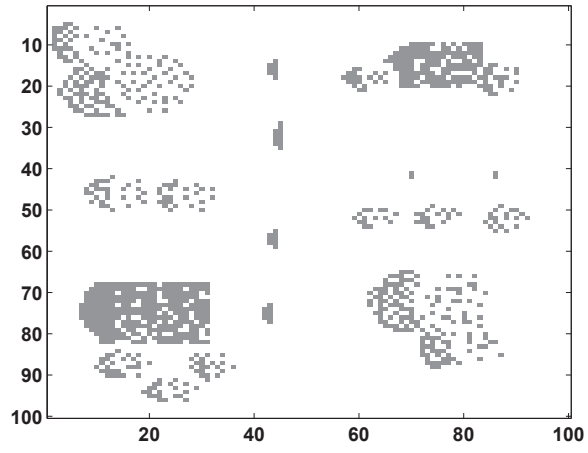

(b) $\beta^{(1)}$

Figure 4.25: Initial estimates for time-1 shadow coefficients using bottom $10 \%$ of spectral norm pixels for MODTRAN data

in Figures 4.2 and 4.3, this shadow initialization has detected all the shadow pixels. However, several non-shadow structures with low reflectance, such as the rectangular areas with terra cota material reflectance assigned, have inaccurately been detected as shadow pixels as well.

Figure 4.27 displays the RT estimates for time- 1 for the three different reflectance estimation modalities. Figure 4.28 displays the RT estimates for time-2. The RT estimates for time-1 are very close to the true illumination used to generate the data. 


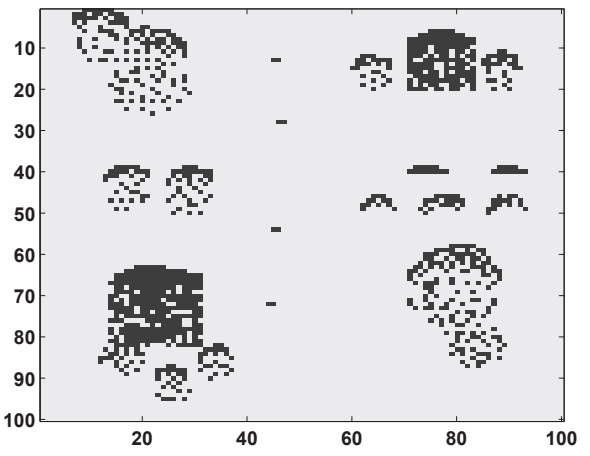

(a) $\tilde{\alpha}^{(2)}$

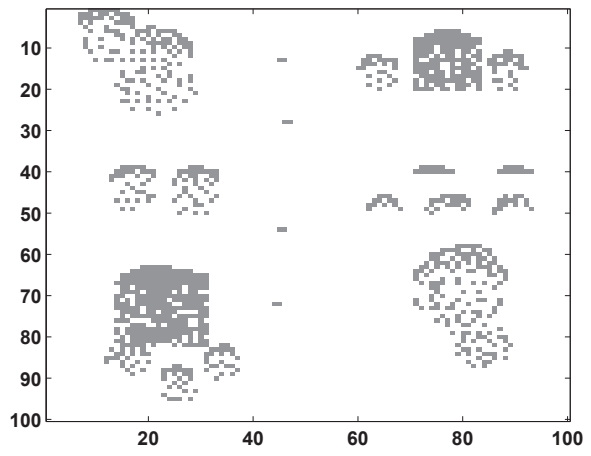

(b) $\beta^{(2)}$

Figure 4.26: Initial estimates for time-2 shadow coefficients using bottom $10 \%$ of spectral norm pixels for MODTRAN data

The direct solar radiance term is overestimated for the time-2 data, whereas the diffuse and path radiance terms are underestimated. This results from the combined inaccurate RT initialization and shadow initialization.

The shadow estimates using the various reflectance estimation modalities are all very similar. Consequently, only the results for the no spectral filtering method are shown. Again, the shadow coefficient estimates correlate strongly with the true shadow conditions. The additional terra cota roof pixels initially identified as shadow pixels still possess low shadow coefficients. This simply results from the ambiguity between low reflectance materials and shadows.

Figure 4.31 displays the empirical ROC curve performance using the $10 \%$ shadow initialization method. The performance of the MB methods in this case is degraded relative to that resulting from the $5 \%$ shadow initialization method. Examination of the detection statistic images provides further insight into clutter suppression performance, sources of false alarms, and missed detections. Figure 4.32 displays the 


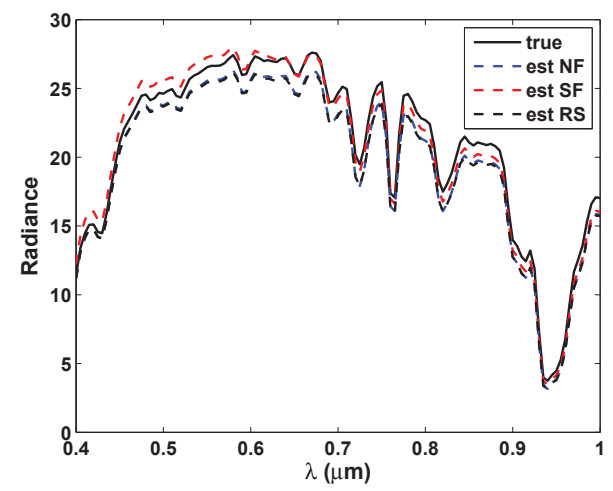

(a) $\boldsymbol{\tau} \odot \mathbf{L}_{s}$

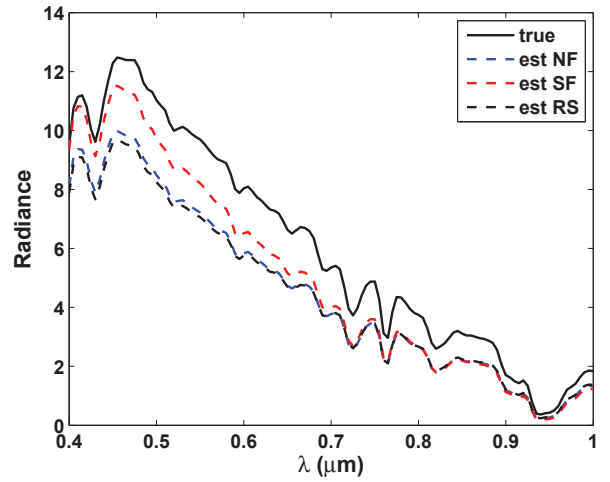

(b) $\boldsymbol{\tau} \odot \mathbf{L}_{d}$

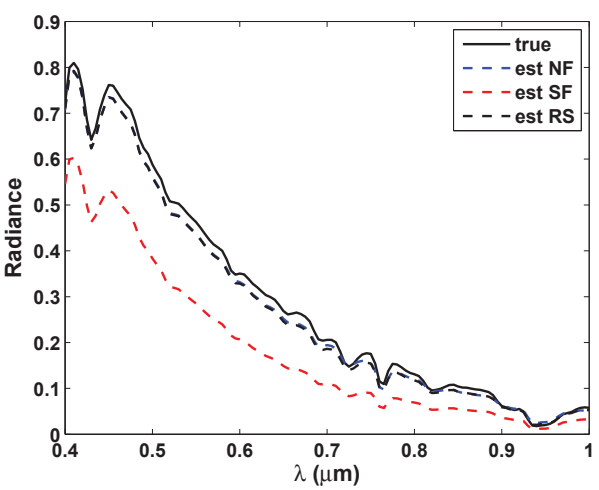

(c) $\mathbf{L}_{p}$

Figure 4.27: RT estimates for time-1 MODTRAN data for different reflectance estimation modalities using $10 \%$ shadow initialization. 


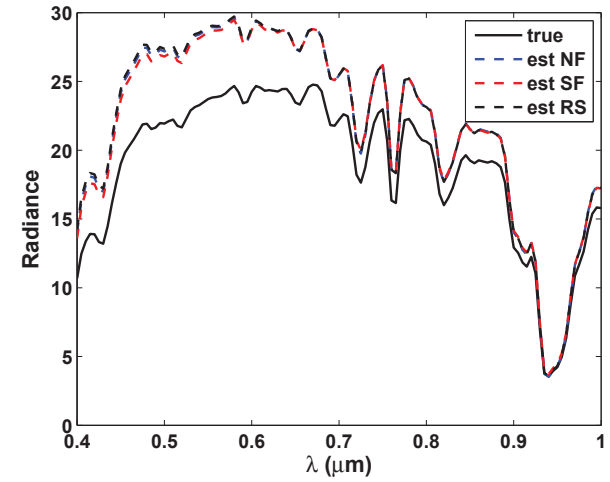

(a) $\boldsymbol{\tau} \odot \mathbf{L}_{s}$

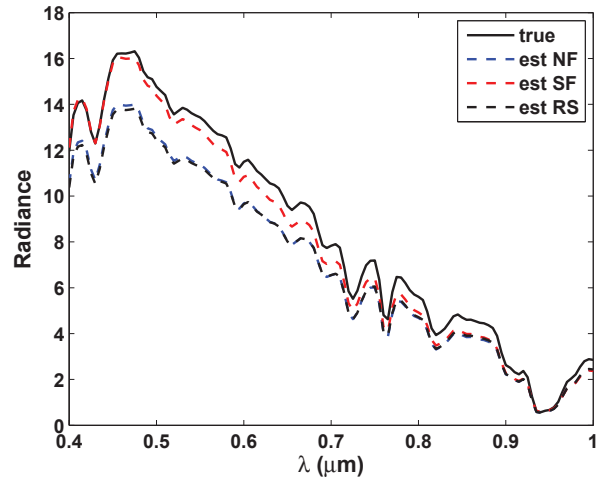

(b) $\boldsymbol{\tau} \odot \mathbf{L}_{d}$

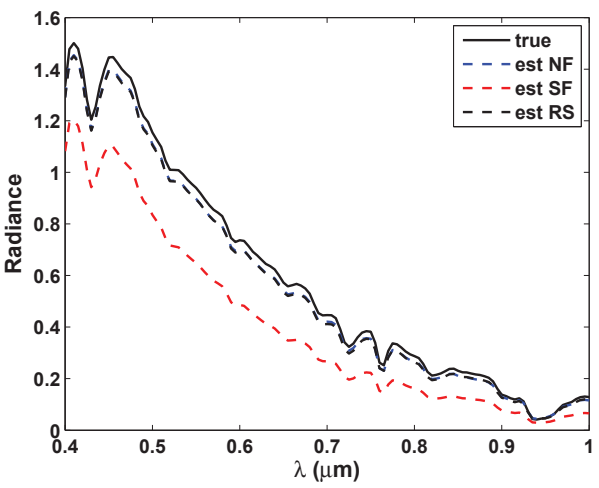

(c) $\mathbf{L}_{p}$

Figure 4.28: RT estimates for time-2 MODTRAN data for different reflectance estimation modalities using $10 \%$ shadow initialization. 


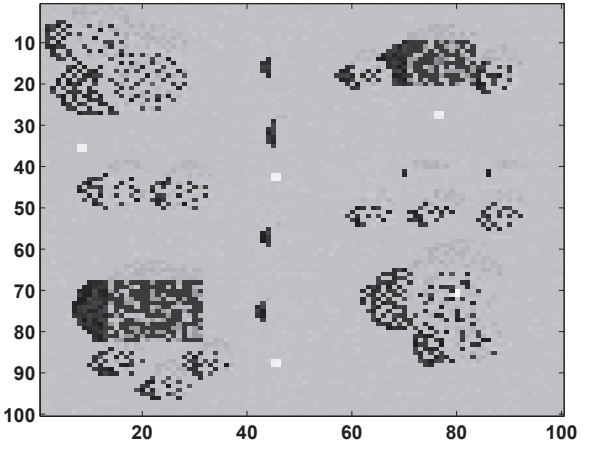

(a) $\hat{\tilde{\alpha}}^{(1)}$

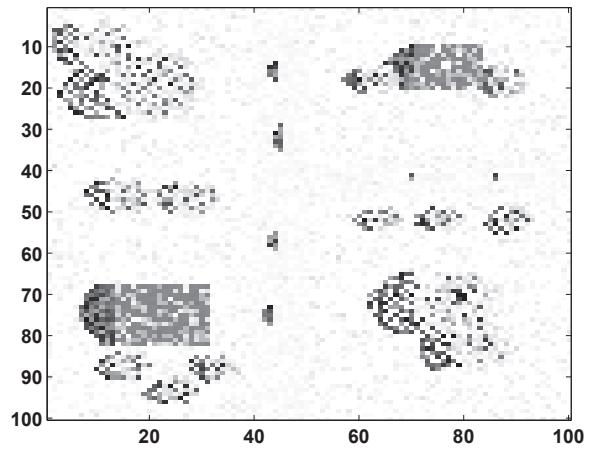

(b) $\hat{\beta}^{(1)}$

Figure 4.29: Estimated shadow coefficients for time-1 data using 10\% shadow initialization for MODTRAN data

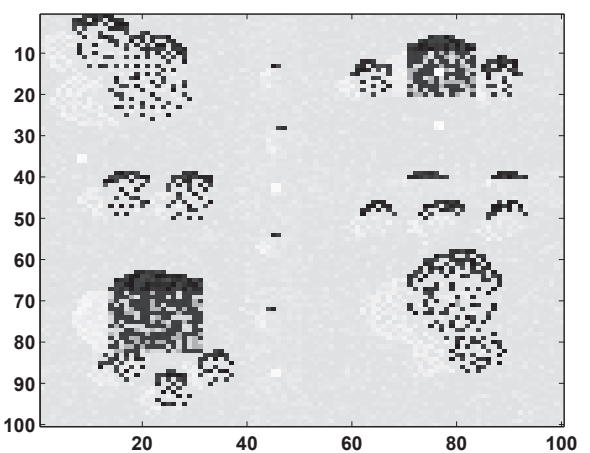

(a) $\hat{\tilde{\alpha}}^{(2)}$

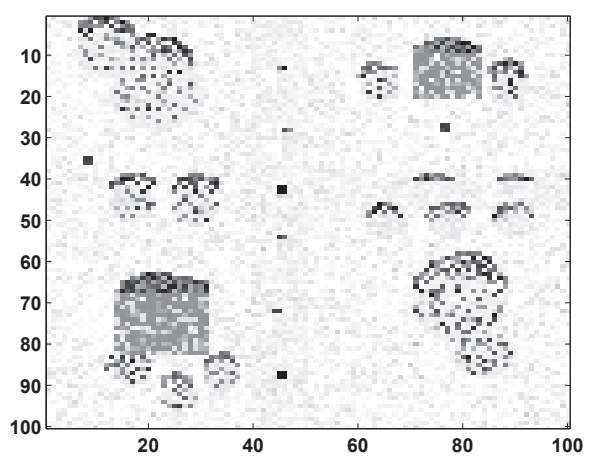

(b) $\hat{\beta}^{(2)}$

Figure 4.30: Estimated shadow coefficients for time-2 data using 10\% shadow initialization for MODTRAN data 


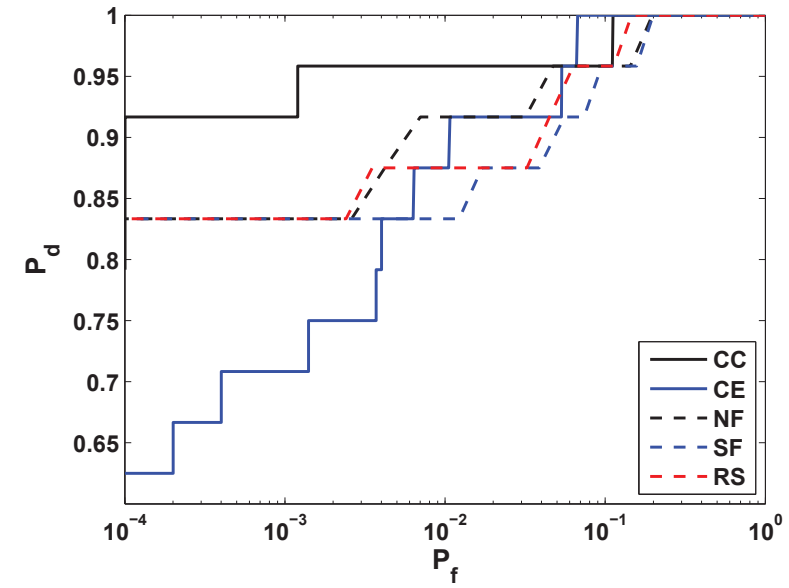

Figure 4.31: Empirical ROC curve performance for different reflectance estimation modalities using $10 \%$ shadow initialization in comparison with $\mathrm{CC}$ and $\mathrm{CE}$ algorithms for MODTRAN data

detection statistic images for the three reflectance modalities of the MB method. The dynamic range of the images is again stretched between 0 and $\mu+7 \sigma$ where $\mu$ is the sample mean of the detection statistic and $\sigma$ is the sample standard deviation. In comparison with the detection statistic images associated with the $5 \%$ shadow initialization method shown in Figure 4.23, there is a reduction in detection performance from increased target suppression associated with the terra cota change target discussed earlier.

The detection performance of the MB change detection algorithm is examined when the true RT and shadow coefficients are used for initialization. The resulting final estimates of the RT terms and shadow coefficients are essentially the same as those used for initialization. Figure 4.33 displays the ROC performance for this case, which is identical to that obtained using the $5 \%$ shadow initialization. This result suggests that the detection performance is not limited by estimation performance, 


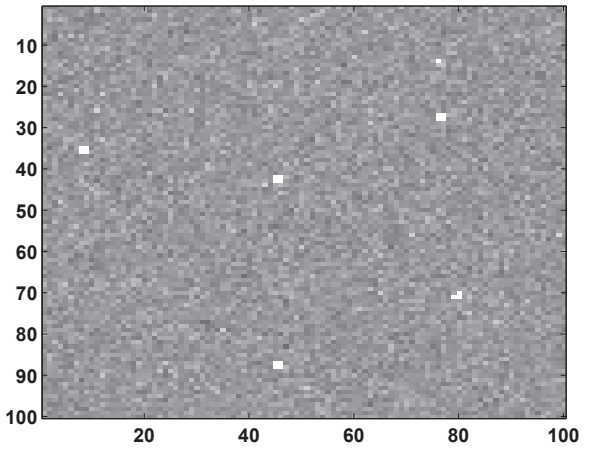

(a) No reflectance filtering

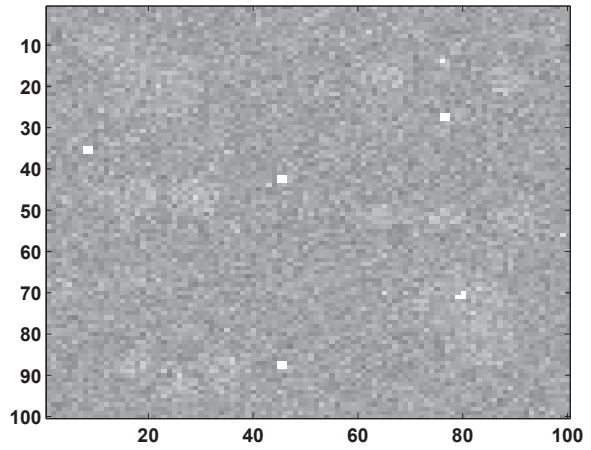

(b) Reflectance filtering

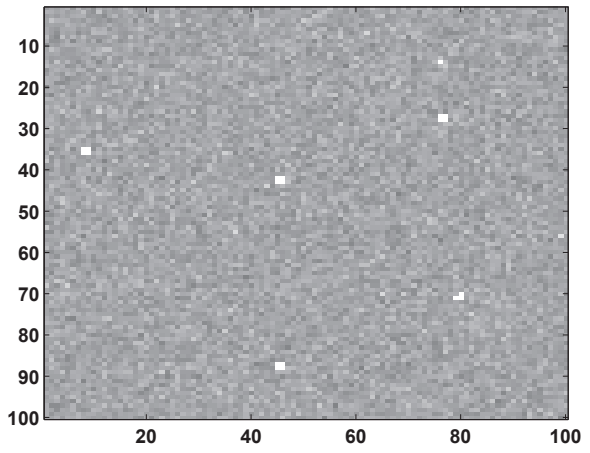

(c) Reflectance subspace

Figure 4.32: Comparison of detection statistic images for different MB reflectance modalities using 10\% shadow initialization for MODTRAN data

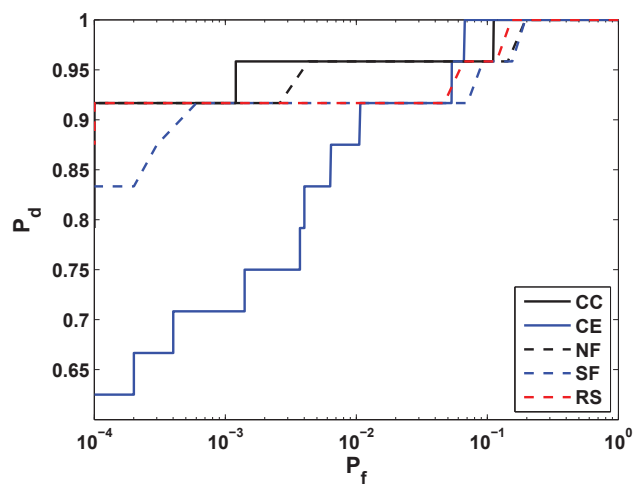

Figure 4.33: Empirical ROC curve performance for different reflectance estimation modalities using true initialization in comparison with $\mathrm{CC}$ and $\mathrm{CE}$ algorithms for MODTRAN data 
but rather by the separability of the change targets themselves as related to change in material reflectance. The ROC performance for all three initialization cases is nearly the same. The small differences in performance are attributed to detection performance associated with the terra cota/red brick change pixels.

\section{SCR Results}

In order to assess the detection performance more fully, the signal-to-clutter statistics associated with the detector outputs are examined. Figures 4.34, 4.35, and 4.36 display the SCR as defined in (2.62) for each target pixel in the scene for the different initialization cases. Figure 4.37 displays the SCR results for CC and CE. Additionally, the average SCR results are provided in Table 4.1. Several interesting observa-

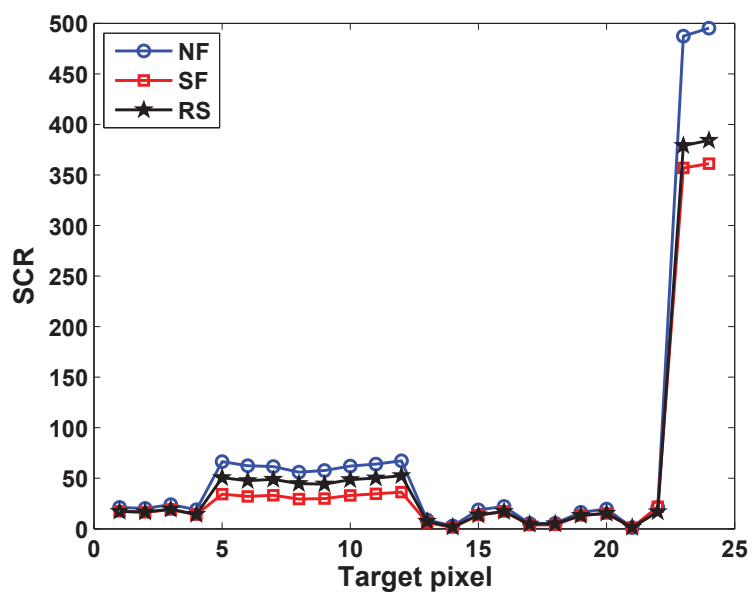

Figure 4.34: Comparison of SCR for different MB reflectance modalities using 5\% shadow initialization for MODTRAN data

tions can be made concerning the SCR results. First, the SCR for the $\mathrm{CC}$ and $\mathrm{CE}$ algorithms is markedly worse than the MB methods. However, CC still manages 


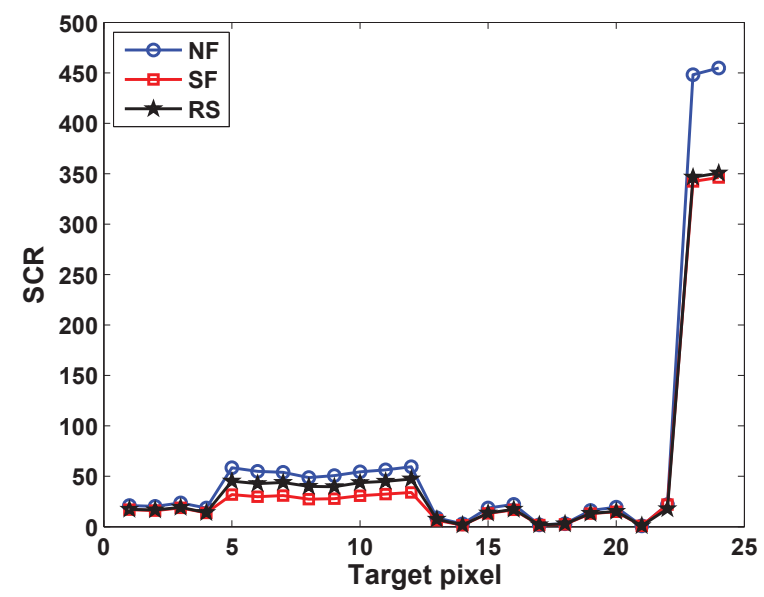

Figure 4.35: Comparison of SCR for different MB reflectance modalities using $10 \%$ shadow initialization for MODTRAN data

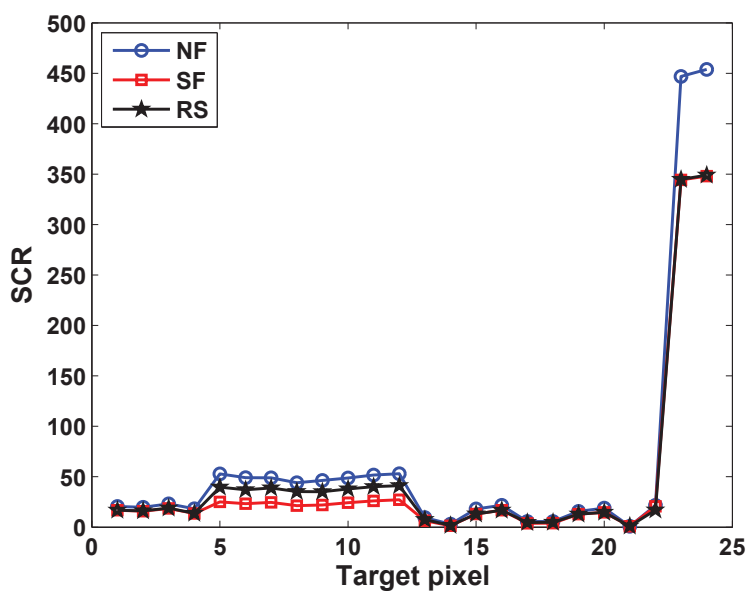

Figure 4.36: Comparison of SCR for different MB reflectance modalities using true initialization for MODTRAN data 


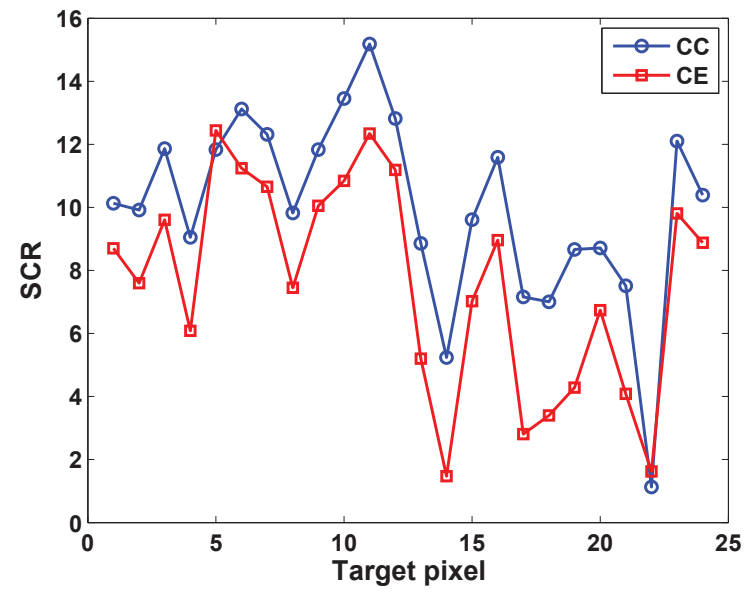

Figure 4.37: Comparison of SCR for CC and CE for MODTRAN data

to achieve high ROC performance while CE performs relatively poorly even though the SCR characteristics are very similar. This trait suggests that the change targets detected with $\mathrm{CC}$ but not in $\mathrm{CE}$ are very close to the edge of the detection statistic distribution for the non-change pixels. With higher data noise, these targets may not be detected with the CC method. Additionally, the mean and standard deviation of the background (non-change) pixels for the MB methods do not change significantly for different initializations. The change in SCR for the methods results mainly from the change in the mean change target signal $\hat{\mu}_{t}$.

\section{Impact of RT Subspace Dimensionality}

The impact of the RT subspace dimensionality $I$ on detection performance is examined. In theory, an optimal value for $I$ exists achieving a balance between minimizing the amount of subspace approximation error while at the same time limiting the degrees of freedom of the RT terms and the number of parameters to estimate. 


\begin{tabular}{|c|c|c|c|c|c|}
\hline Algorithm & $\hat{\mu}_{t}$ & $\hat{\mu}_{b}$ & $\hat{\mu}_{t}-\hat{\mu}_{b}$ & $\hat{\sigma}_{b}$ & $\hat{\mu}_{S C R}$ \\
\hline CC & 296.60 & 120.56 & 176.04 & 17.66 & 9.97 \\
\hline CE & 255.08 & 120.66 & 134.42 & 17.68 & 7.60 \\
\hline NF-5\% & 1200.10 & 119.11 & 1081.00 & 15.38 & 70.25 \\
\hline NF-10\% & 1106.00 & 119.18 & 986.83 & 15.39 & 64.13 \\
\hline NF-True & 1078.5 & 119 & 959.5 & 15.37 & 62.4 \\
\hline SF-5\% & 1175.80 & 202.73 & 973.03 & 20.39 & 47.72 \\
\hline SF-10\% & 1133.50 & 202.97 & 930.56 & 20.45 & 45.5 \\
\hline SF-True & 1084.5 & 202.6 & 881.86 & 20.24 & 43.57 \\
\hline RS-5\% & 1296.90 & 200.67 & 1096.20 & 20.01 & 54.77 \\
\hline RS-10\% & 1204.00 & 200.73 & 1003.30 & 20.01 & 50.13 \\
\hline RS-True & 1166.2 & 200.65 & 965.57 & 20.00 & 48.28 \\
\hline
\end{tabular}

Table 4.1: SCR for various algorithms using MODTRAN data set

More degrees of freedom may allow for the suppression of change targets within the scene by manipulation of the less significant eigenvectors of the RT subspace. Two cases are examined here using the baseline no spectral filtering method with the $5 \%$ shadow initialization case studied above. In the first case, the RT basis coefficients are left unbounded, which will allow the less significant eigenvectors to potentially take on larger values than expected. In the second case, the basis coefficients are bounded as they normally would be in operation of the MB algorithm. For both cases, the subspace dimensionality $I$ is varied from 1 to 50 and the mean SCR is calculated on the output of the MB change detection method. Figure 4.38 displays the SCR results for both cases. The results demonstrate a decrease in SCR performance for both cases when the subspace dimension is less than 4. However, unlike the unbounded case, the performance of the bounded $\mathrm{MB}$ algorithm does not decrease as the subspace dimension becomes large. The basis bounds effectively restrict the impact of the less significant subspace eigenvectors preventing manipulation of these terms to suppress 


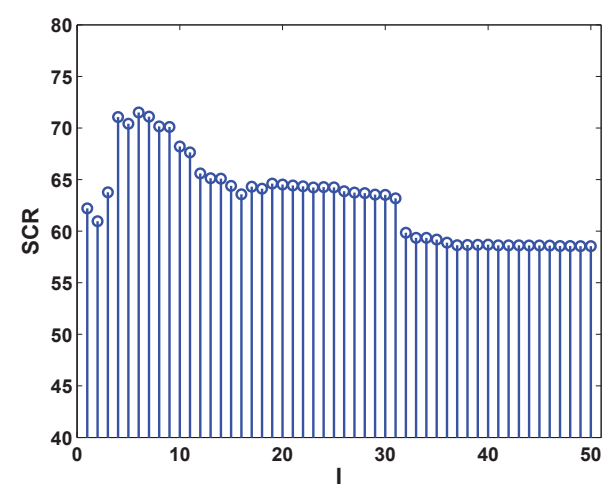

(a) Unbounded

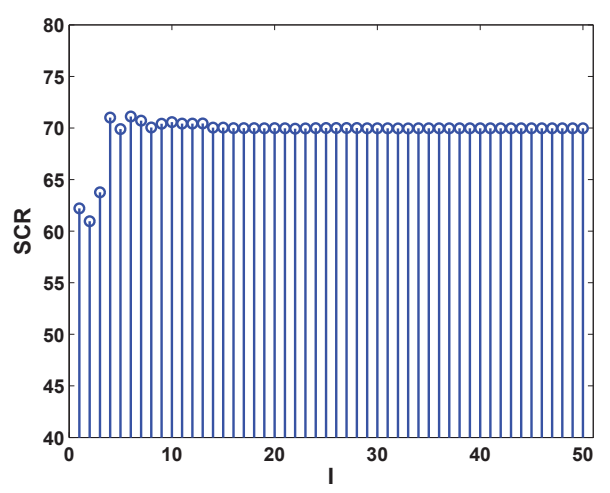

(b) Bounded

Figure 4.38: SCR as a function of subspace dimensionality $I$ for MODTRAN data. In (a), no explicit bounds are placed on the basis coefficients, whereas MODTRAN realizations are used to place bounds on the coefficients for (b)

change targets. However, the results still demonstrate that limited, if any, gain in performance is achieved by using a larger subspace dimension. Consequently, increasing the subspace dimension simply increases the number of parameters to estimate in the data model.

\subsection{AFRL Tower Data}

Concept validation for the proposed change detection method on the synthetic MODTRAN imagery is extended by testing on real imagery. Application of the MB change detection method to real imagery is challenging for a number of reasons. The absolutely calibrated data model does not incorporate any terms to account for calibration error, which can be significant in real imagery. Additionally, certain data sets may demonstrate nonlinear scattering and adjacency effects not captured in the model. Consequently, results using real imagery are much more significant than those obtained using simulated data. In this section, the AFRL in-house hyperspectral 
data discussed in Chapter 3 is used, which utilizes an absolute radiometric calibration producing data with radiometric units. The data calibration process is fully known for this data set, which allows for testing of the uncalibrated and relatively calibrated data models developed as well. Further specifications of the imaging sensor are provided in regards to the data sets used here for change detection testing. After discussing the data, the same change detection tests are applied as for the synthetic imagery of the previous section. Additionally, the uncalibrated and relatively calibrated data models and 4-step AO algorithms are applied to these data.

\subsubsection{Data Summary}

The specifications of the AFRL VNIR pushbroom style imaging spectrometer are provided in Table 4.2. The data were collected in a controlled manner from a tower

\begin{tabular}{|l|c|}
\hline Platform: & Pan and tilt assembly \\
\hline Tower altitude: & $313.94 \mathrm{~m} \mathrm{ASL}(25.9 \mathrm{~m}$ AGL) \\
\hline IFOV: & $0.24 \mathrm{mrad}$ \\
\hline Wavelength range: & $0.46-0.9 \mu \mathrm{m}$ \\
\hline Spectral bands: & 124 \\
\hline Spectral sampling: & $3.6 \mathrm{~nm}$ \\
\hline Spectral Resolution: & 8nm (avg) \\
\hline
\end{tabular}

Table 4.2: Air Force Research Laboratory in-house sensor information

with the sensor mounted on a pan and tilt assembly to help eliminate misregistration between image pairs. The tower data collection occurred on Wright Patterson Air Force Base (WPAFB) $\left(39^{\circ} 46^{\prime} 42^{\prime \prime} \mathrm{N}, 8^{\circ} 5^{\prime} 10^{\prime \prime} \mathrm{W}\right.$ - WGS84) from August 2005 to May 2006 during which more than 100 data sets were collected. The primary purpose of the data collection was to characterize diurnal and seasonal background changes 
and their impact upon target/change detection algorithms [54, 56]. Measurements previously taken with a GPS provide a scene elevation of nominally $288 \mathrm{~m}$ above sea level (ASL) and camera elevation of around $25.9 \mathrm{~m}$ above ground level (AGL). The sensor stared at a declination angle of about $6^{\circ}$ to image the scene resulting in a slant range of $247.8 \mathrm{~m}$. For this study, focus is given to two data sets collected on October 14, 2005 and October 17, 2005 respectively. Figure 4.39 displays color images of the two data sets generated using red, green, and blue bands from the data cubes. The

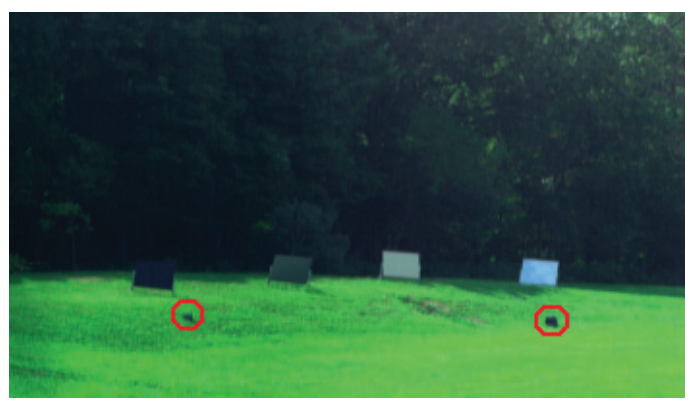

(a) Time-1 data collected 14, 2005 at 13:11 EDT

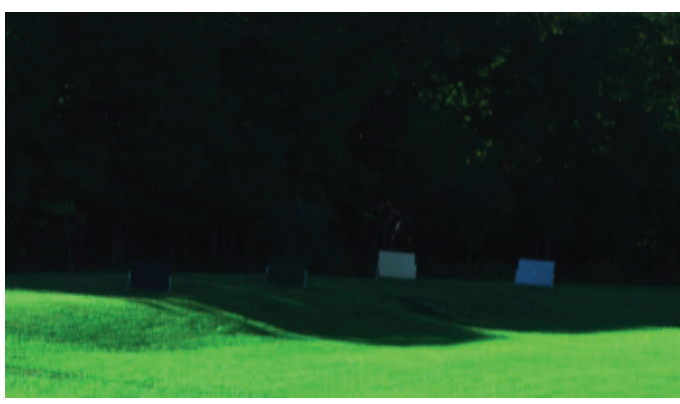

(b) Time-2 data collected October 17, 2005 at 10:57 EDT

Figure 4.39: Change pair collected with AFRL in-house, tower-based VNIR hyperspectral sensor

scene is comprised of mostly grass and trees with four coated aluminum panels (black, green, tan, gray) placed along the treeline. The October 14 data set was collected around solar noon whereas the October 17 data set was collected around 1100 EST. Consequently, significant shadow variation between the data sets exists. Using the provided collection time information and latitude/longitude of the location, the solar position at the time of each collection is calculated. This information is provided in Table 4.3 where the solar elevation (SE) is defined as degrees up from the horizon 
(90-Solar Zenith) and the solar azimuth (SA) is defined as degrees clockwise from North. Two changes of interest have been identified in the scene. These changes

\begin{tabular}{|c|c|c|c|}
\hline Date & Acquisition Time (hh:mm EDT) & SE & SA \\
\hline October 14, 2005 & $13: 11$ & $41.80^{\circ}$ & $176.29^{\circ}$ \\
\hline October 17, 2005 & $10: 57$ & $30.60^{\circ}$ & $137.70^{\circ}$ \\
\hline
\end{tabular}

Table 4.3: AFRL in-house data collection information

correspond to two small tarp bundles that have been removed from time-1 to time-2. The smaller of the two tarps (on left) is a green tarp material whereas the larger tarp is camouflage (a combination of dark green, brown, and black pigments). The challenge for the algorithm is to detect these two small changes while ignoring the shadow variations. These changes are particularly challenging to detect as both have very low reflectance as demonstrated in Figure 4.40. Consequently, the algorithm can

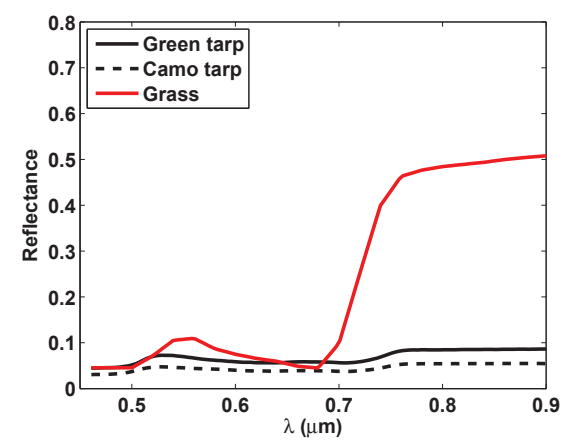

Figure 4.40: Lab reflectance spectra for green tarp, camouflage tarp, and grass for AFRL data

easily confuse these materials as shadows. Additionally, the location of the larger tarp 
corresponds to a shadow location in the time-2 scene, meaning the difference between the two signals will be relatively small. To get a better understanding of the difficulty of the task, the measurements from the time- 1 and time- 2 scenes are displayed for a pixel corresponding to the small green tarp location and the larger camouflage tarp location in Figure 4.41. The change in the measured signal for the camouflage tarp

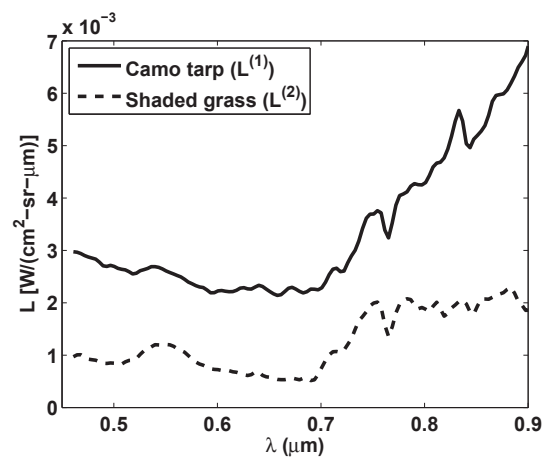

(a) Camouflage tarp

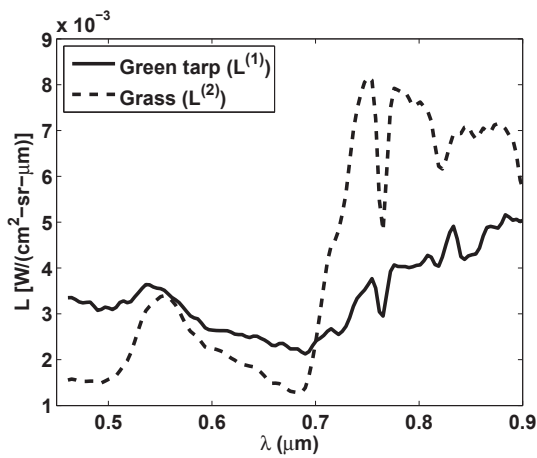

(b) Green tarp

Figure 4.41: Comparison of measured signal associated with change pixel locations at time- 1 and time-2 for AFRL data

pixel is rather subtle because the tarp itself has low reflectance and the corresponding pixels at time-2 are in shade. The two signals can nearly be matched using a simple gain or offset. Consequently, this change will be difficult to detect. The change in signal associated with the green tarp (green tarp at time-1 to sunlit grass at time-2) is more significant.

\subsubsection{Results using Absolutely Calibrated Data Model}

The MB algorithm is applied to this data set for a number of different configurations and initializations. For these cases, an RT subspace dimensionality of $I=10$ 
is used as in the previous case due to a similar wavelength range. The initialization for this data set is more complicated than the previous artificial MODTRAN scene because the true atmospheric conditions are unknown. Additionally, the panels, treeline, and portions of the grass are not flat and the slant viewing geometry associated with this data make the initialization of the cosine projection term $\cos \theta_{m, n}$ associated with $\tilde{\alpha}$ difficult. Consequently, the initialization $\theta_{m, n}=0$ is used due to lack of further knowledge. Additionally, due to this slant viewing geometry, the path from sensor-to-scene varies in length across the scene, which deviates from the physical model used and may complicate detection/estimation performance. The RT conditions are initialized using the known solar position and sensor altitude. MODTRAN is used to generate RT terms using the standard mid-latitude summer atmospheric profile with $20 \mathrm{~km}$ visibility for both time- 1 and time- 2 and using the default water vapor column associated with the atmospheric model. In order to demonstrate the challenges associated with initializing RT parameters, the initial estimates of the expected radiance for the grass region using the initial estimates for the RT conditions are examined using a laboratory grass reflectance spectrum displayed in Figure 4.40. The grass reflectance spectrum is assumed to be a relatively accurate representation of the healthy grass within the scene. Figure 4.42 displays the initial estimates for a grass pixel as compared with the actual measurements for that pixel assuming both the direct and diffuse shadow coefficients are 1. The initial radiance estimates are lower than the true measurements. This discrepancy can result from various phenomena. The visibility of the true atmosphere can be greater than the initial estimate used. Additionally, the grass could demonstrate specular reflectance characteristics that are not captured in the physical model, making the grass spectrum assumed in 


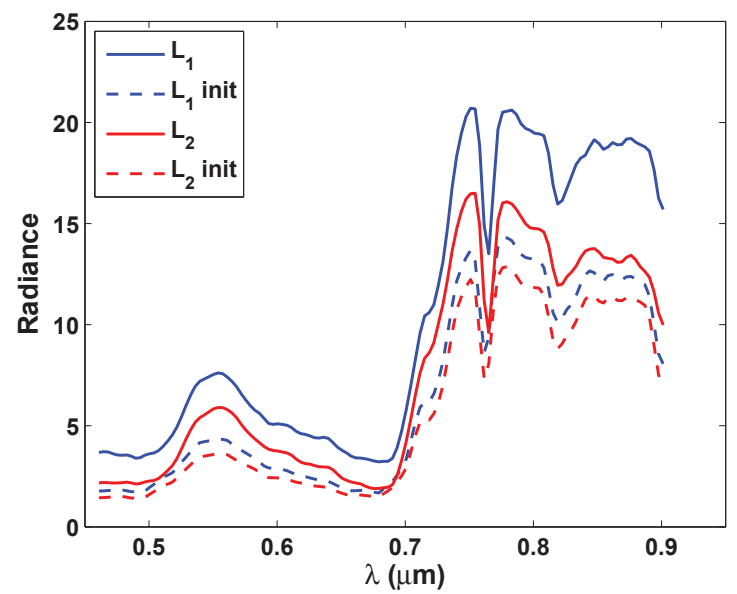

Figure 4.42: Initial estimates of radiance for grass pixel as compared with true measurements for AFRL data

the comparison a poor estimate for the apparent spectral reflectance. Due to the slant viewing geometry and treeline-backed scene, the radiance reaching the sensor may be greater than expected due to scattering introduced by the treeline region, which is a very complex effect to incorporate into the model. The detection and estimation results using this initialization will be examined as this represents the most realistic operational scenario for the algorithm and data set.

The estimation and detection results are compared for the $\mathrm{MB}$ algorithm run in the three different reflectance estimation modalities as in the previous case. The algorithm is run using 20 iterations. Again, this number of iterations was determined by visually examining changes in state estimates and ROC performance and analysis of the convergence criteria as a function of iteration. Figure 4.43 displays the convergence criteria given in (2.56) and (2.57) for one particular case for the AFRL data. No appreciable changes in detection performance or state estimates is observed 


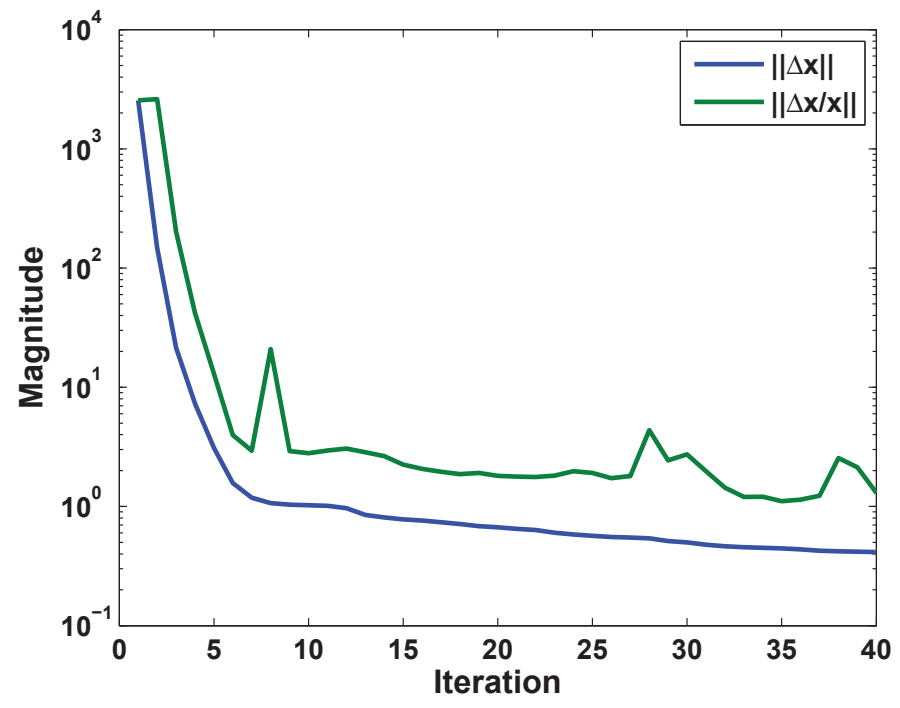

Figure 4.43: Convergence criteria as a function of iteration for AFRL data

for iterations beyond 20. For the reflectance smoothing implementations of the algorithm, a Bartlett window of length 5 is used as the data is relatively smooth to begin with due to lower spectral resolution. As before, a reflectance subspace dimension of $I_{r}=40$ is used when implementing the Legendre subspace representation. Radiative transfer parameter and shadow coefficient estimation error cannot be quantified as the true values of these parameters for the scene are unknown. However, spectral laboratory reflectance spectra of the panels and a nominal grass spectrum are available for comparison.

\section{$5 \%$ Shadow Initialization}

The histogram threshold method is used to detect the bottom $5 \%$ of spectral norm pixels in both the time- 1 and time- 2 scenes. These pixels are detected as shadow pixels and initialized with $\tilde{\alpha}=0.1$ and $\beta=0.5$. Figures 4.44 and 4.45 display the initial 
shadow coefficient estimates for the time-1 and time-2 data respectively. Shadow

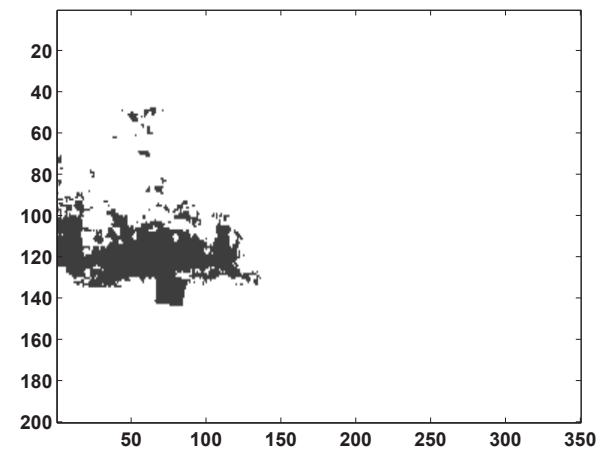

(a) $\tilde{\alpha}^{(1)}$

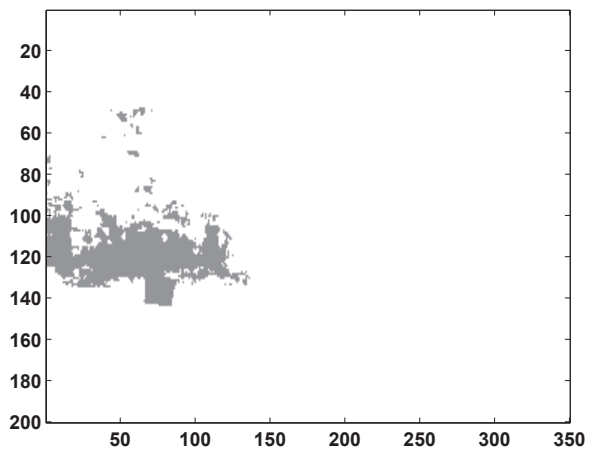

(b) $\beta^{(1)}$

Figure 4.44: Initial estimates for time-1 shadow coefficients using bottom $5 \%$ of spectral norm pixels for AFRL data

truth does not exist for these data, consequently, a quantifiable comparison to truth is not possible. The initial shadow estimates detected by histogram thresholding are the deep shadows present in the treeline, along with the black panel in the scene. Detection and estimation results are obtained using these initial shadow and RT estimates. While no quantifiable assessment of the estimation error of the MB algorithm for these terms is possible, the estimated RT terms can be compared with the various MODTRAN realizations used to create the RT subspace. The realization that is closest to the estimated RT term in a sum-squared-error sense provides a sanity check for the validity of the estimates based on the nominal collection conditions. For this initialization, the estimated time-1 RT parameters are nearest to several realizations generated using a tropical atmosphere with $20 \mathrm{~km}$ visibility and $0.2 \%$ water column. This essentially corresponds to using a model atmosphere with high water content, 


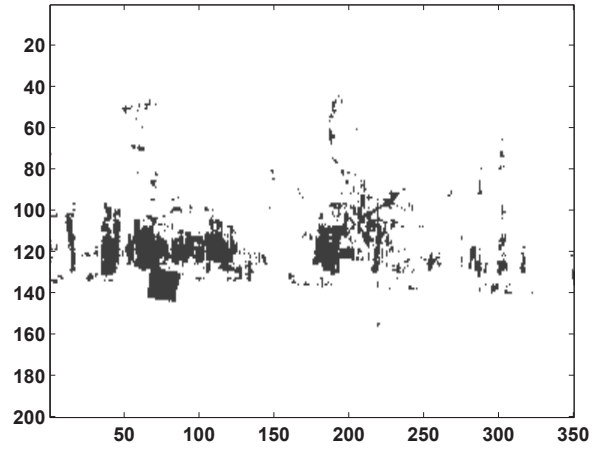

(a) $\tilde{\alpha}^{(2)}$

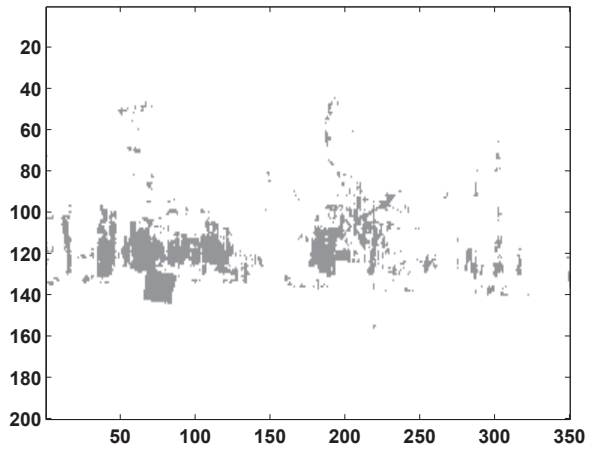

(b) $\beta^{(2)}$

Figure 4.45: Initial estimates for time-2 shadow coefficients using bottom $5 \%$ of spectral norm pixels for AFRL data

but then reducing the water vapor content and increasing the visibility. Figure 4.46 displays the RT estimates for time-1 for the three different reflectance estimation modalities. Conversely, the estimated time-2 RT conditions are closest to MODTRAN realizations generated using a standard U.S. atmospheric model with $30 \mathrm{~km}$ visibility and nearly double the default water vapor column. Figure 4.47 displays the RT estimates for time-2 for the three different reflectance estimation modalities compared with the nearest MODTRAN realization.

The shadow coefficient estimates using the various reflectance estimation modalities are all very similar. Consequently, only the results for the baseline non-filtering method are shown. Figures 4.48 and 4.49 display the shadow coefficient estimates for the time- 1 and time- 2 data. The time- 1 shadow estimates are similar to the initialization used. While the scene itself does not possess many shadows in the grass region, shadow variation in the treeline region is expected but is not visible in the 


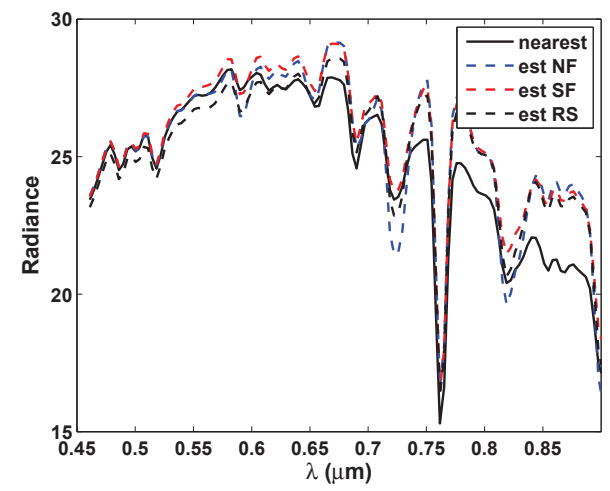

(a) $\boldsymbol{\tau} \odot \mathbf{L}_{s}$

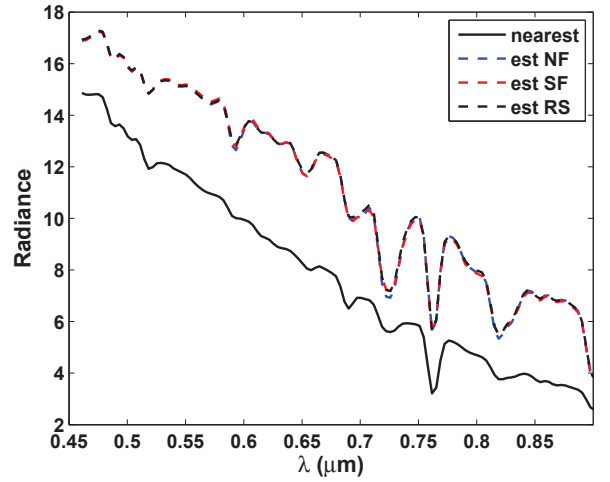

(b) $\boldsymbol{\tau} \odot \mathbf{L}_{d}$

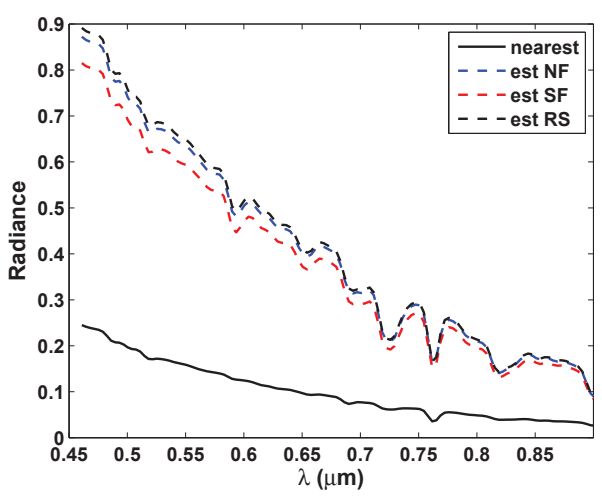

(c) $\mathbf{L}_{p}$

Figure 4.46: RT estimates for time-1 AFRL data for different reflectance estimation modalities using $5 \%$ shadow initialization. 


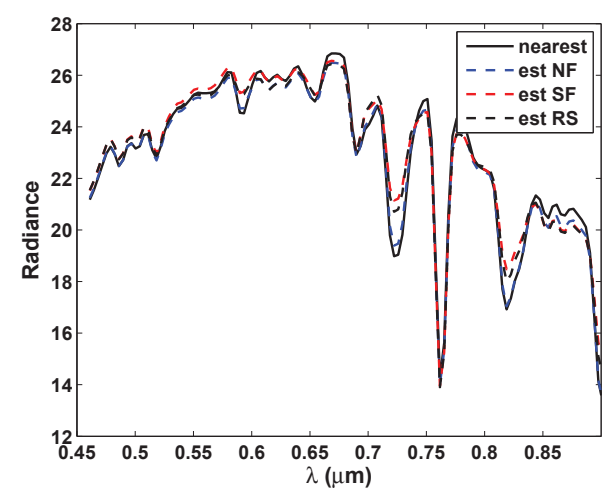

(a) $\boldsymbol{\tau} \odot \mathbf{L}_{s}$

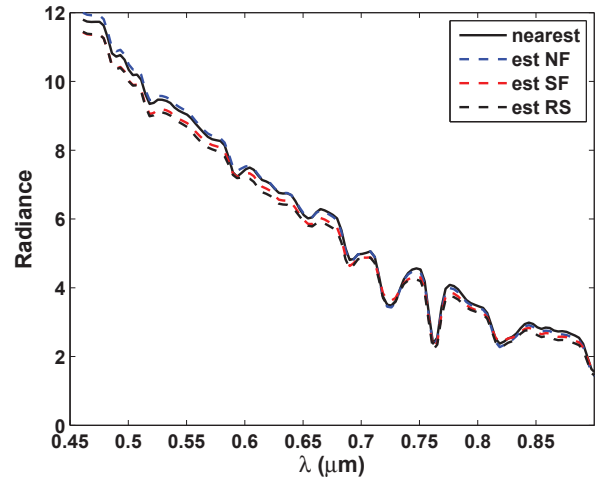

(b) $\boldsymbol{\tau} \odot \mathbf{L}_{d}$

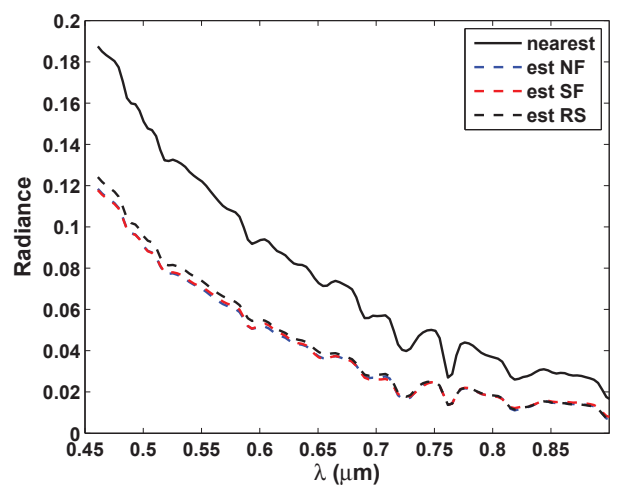

(c) $\mathbf{L}_{p}$

Figure 4.47: RT estimates for time-2 AFRL data for different reflectance estimation modalities using $5 \%$ shadow initialization 


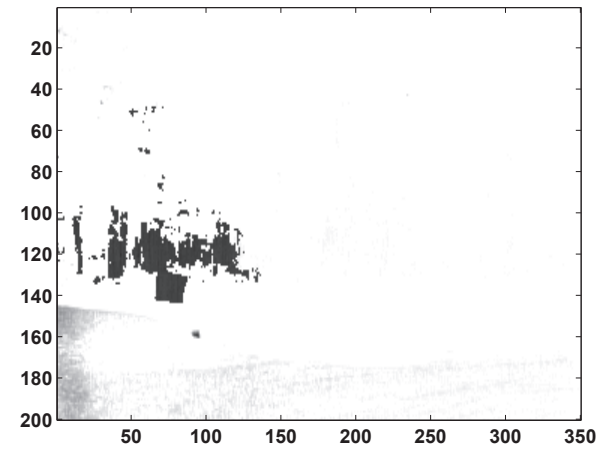

(a) $\hat{\tilde{\alpha}}^{(1)}$

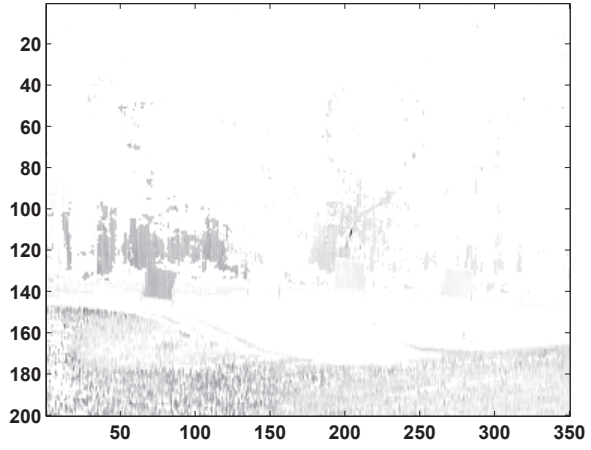

(b) $\hat{\beta}^{(1)}$

Figure 4.48: Estimated shadow coefficients for time-1 data using 5\% shadow initialization for AFRL data

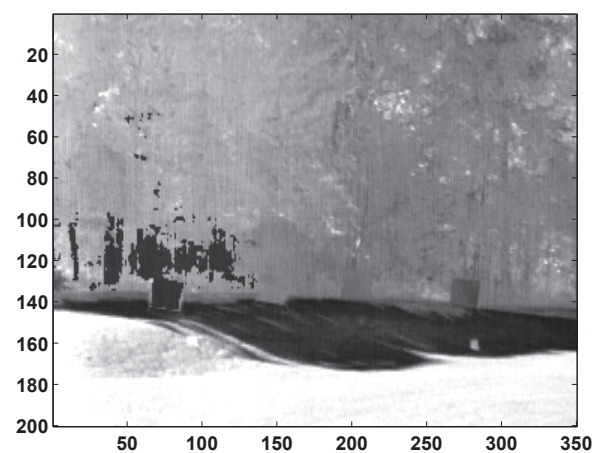

(a) $\hat{\tilde{\alpha}}^{(2)}$

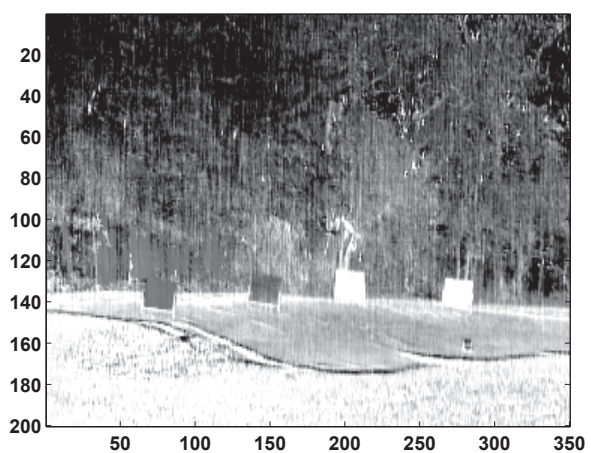

(b) $\hat{\beta}^{(2)}$

Figure 4.49: Estimated shadow coefficients for time-2 data using 5\% shadow initialization for AFRL data 
time-1 shadow estimates. The low initial time-1 RT estimates provided for the algorithm as demonstrated earlier in Figure 4.42 may produce this result. Since the RT basis coefficients are estimated in the final step of the AO algorithm, the reflectance and shadow coefficient estimates often compensate for any residual measurement error resulting from poor initial estimates of the RT conditions. In a sense, the shadow coefficients are overestimated to compensate for underestimated RT conditions. The shadow estimates for the time-2 data correlate well with the shadows observed in the color imagery.

The residual estimation error resulting from the final state estimates is examined. Figure 4.50 displays the estimated measurement $\hat{L}$ for a grass pixel for both the time1 and time-2 data. Figure 4.51 displays the same for a pixel located on the green

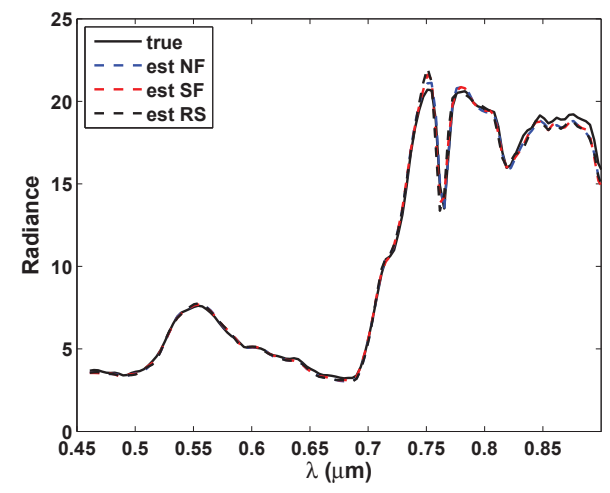

(a) Time-1

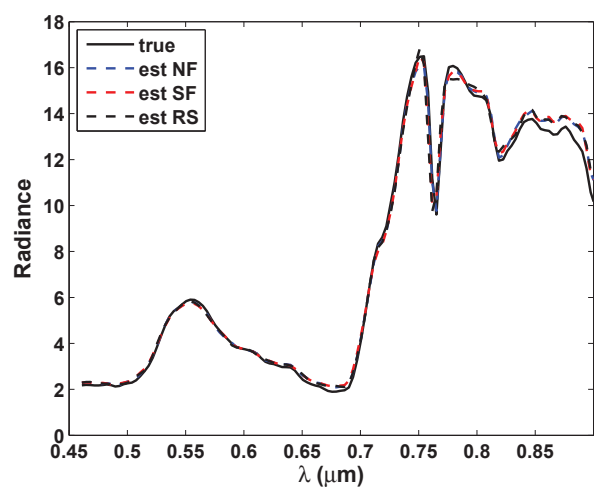

(b) Time-2

Figure 4.50: Comparison of estimated measurement $\hat{L}$ with true measurement $L$ for grass pixel using $5 \%$ shadow initialization for AFRL data

aluminum panel. The optimization error is larger at wavelengths above $0.8 \mu m$ than at lower wavelengths. From previous analysis of this data set, interesting phenomena 


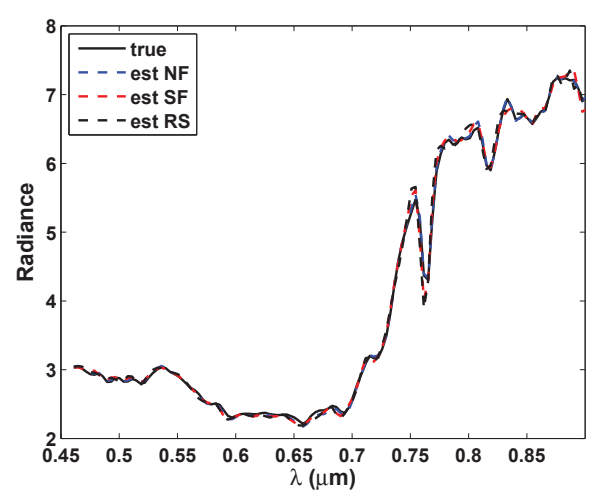

(a) Time-1

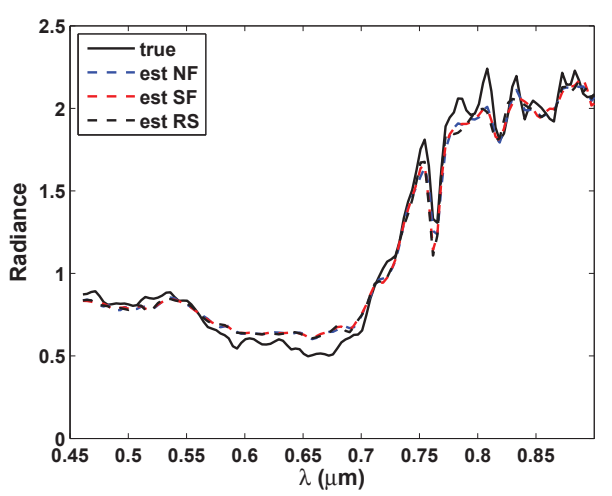

(b) Time-2

Figure 4.51: Comparison of estimated measurement $L$ with true measurement for green panel pixel using 5\% shadow initialization for AFRL data

exist in the data at these higher wavelengths that are not adequately described by the physical model used. This may be related to the scattering affects introduced by the treeline background as discussed earlier. While scattering is typically lower at larger wavelengths, vegetation reflectance is much higher beyond $0.8 \mu m$, which may introduce more adjacency effects. This model mismatch results in residual optimization error at these higher wavelengths. The green panel is located in shadow at time-2, resulting in a low time-2 signal for this material. Figure 4.52 displays the reflectance estimates associated with these pixels in comparison with laboratory truth. The grass reflectance estimates have the same spectral shape as the laboratory spectral signature but vary by a small gain term. The spectral filtering method and reflectance subspace method both provide smoother reflectance estimates. The reflectance estimate of the green panel is much less than the true reflectance signature. The reflectance estimates associated with the panels present a difficult problem in general due to the solar/viewing geometry of the scene along with the geometry of 


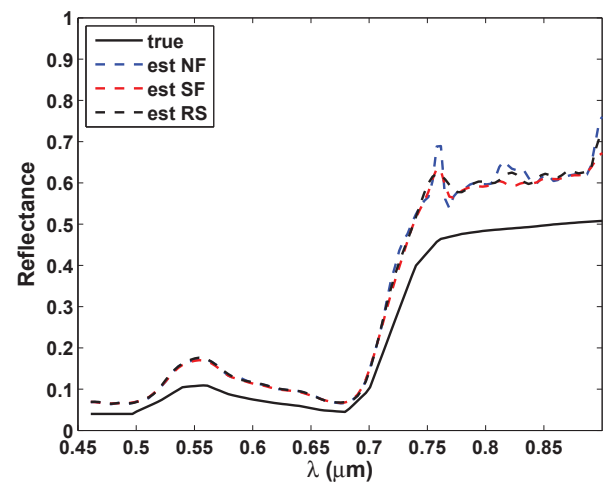

(a) Grass

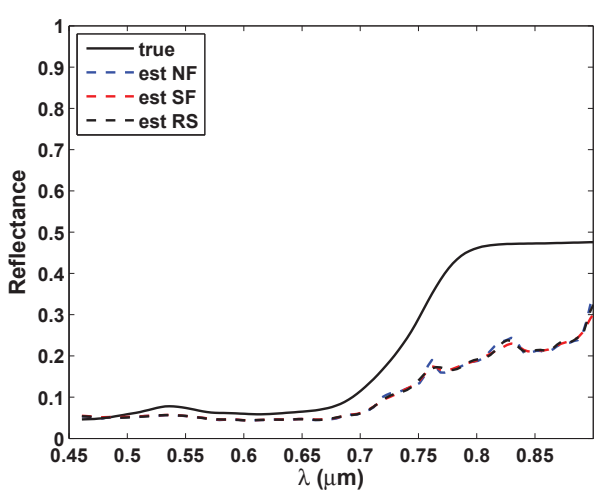

(b) Green panel

Figure 4.52: Comparison of estimated reflectance $\rho$ with laboratory reflectance for grass pixel and green panel pixel using $5 \%$ shadow initialization for AFRL data

the panels and the possible adjacency effects of the treeline. The relative orientation of the panels with regard to the sensor location is much different than the grass and other objects in the scene. Additionally, the panels are located in shadow at time- 2 . As a result, the time- 2 measurements do not provide much information with regard to estimating reflectance parameters because the signals are small. These circumstances allow for relatively inaccurate reflectance estimates that do not produce significant residual optimization error.

Figure 4.53 displays the empirical ROC curve performance of the MB algorithm using the three reflectance estimation modalities in comparison with the performance of the $\mathrm{CC}$ and $\mathrm{CE}$ change detection algorithms discussed. In general, the ROC performance appears poor because all the algorithms fail to detect the more difficult larger camouflage tarp change at low false alarms. However, when comparing solely the detection of the green tarp, the MB methods clearly perform better than the global CC and CE methods. The green tarp change is identified at $P_{d}=0.34$ and $P_{f} \approx 10^{-4}$ 


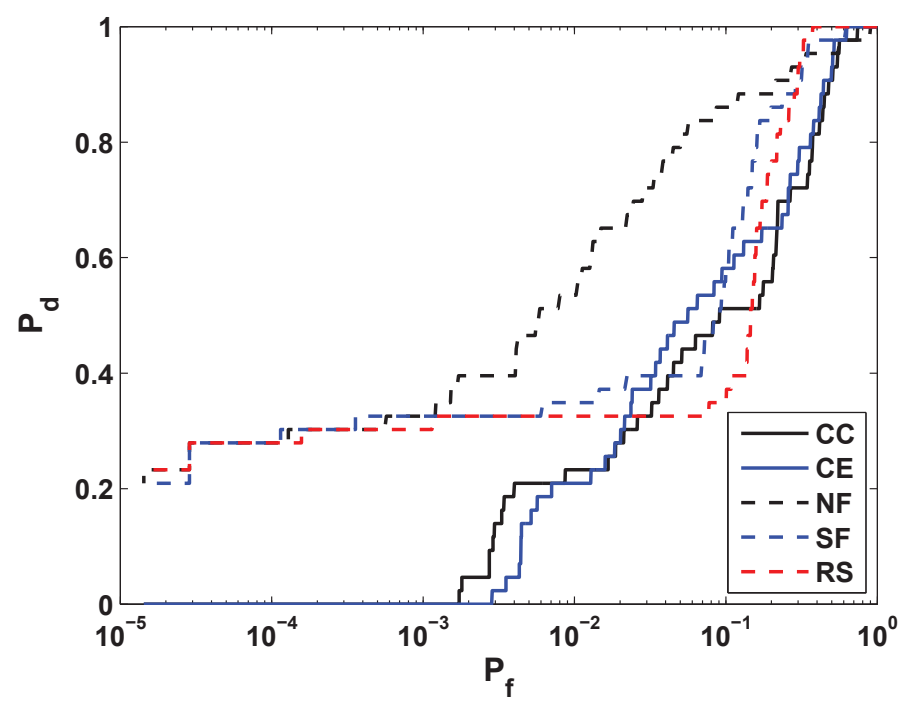

Figure 4.53: Empirical ROC curve performance for different reflectance estimation modalities using $5 \%$ shadow initialization in comparison with $\mathrm{CC}$ and $\mathrm{CE}$ algorithms for AFRL data

with the MB methods, which is at a significantly lower false alarm rate than CC and CE. The MB algorithm with no reflectance filtering additionally achieves improved detection performance for the camouflage tarp change. Examination of the detection statistic images provides further insight into clutter suppression performance, sources of false alarms, and missed detections. Figure 4.54 displays the detection statistic images of the $\mathrm{CC}$ and $\mathrm{CE}$ algorithms respectively. These algorithms demonstrate clutter leakage in the shadow regions as well as in the upper right portion of the tree region. Figure 4.55 displays the detection statistic images for the three reflectance modalities of the MB method. Again, the dynamic range of the images is stretched between 0 and $\mu+7 \sigma$. Visually, the detection statistic image for the MB approach using the reflectance subspace seems to suppress background clutter best, especially in the shadow region. However, the camouflage tarp change is additionally suppressed. 


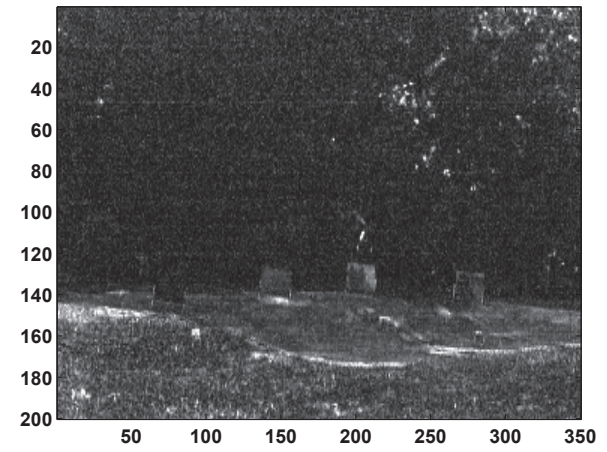

(a) $\mathrm{CC}$

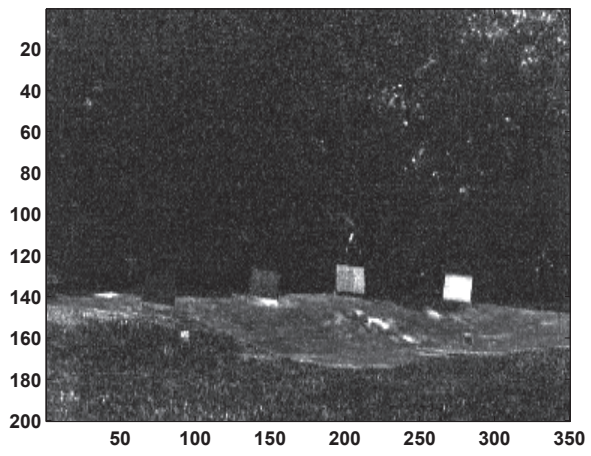

(b) $\mathrm{CE}$

Figure 4.54: Comparison of detection statistic images for $\mathrm{CC}$ and $\mathrm{CE}$ change detection algorithms for AFRL data

All of the MB algorithms possess residual clutter leakage in the lower grass region of the scene. This residual clutter can result from the potential model mismatch associated with treeline scattering discussed earlier.

\section{0\% Shadow Initialization}

The MB methods are repeated using the bottom $10 \%$ of spectral norm pixels in both the time- 1 and time- 2 scenes as shadow pixels. These pixels are detected as shadow pixels and initialized with the same shadow coefficient values as the $5 \%$ case. Figures 4.56 and 4.57 display the initial shadow coefficient estimates for the time-1 and time-2 data respectively. The initial shadow estimates correspond to more of the deep shadows present in the treeline, along with the black panel in the scene. This alternative shadow initialization does not significantly alter the final RT estimates from the previous initialization case, as demonstrated in Figures 4.58 and 4.59. 


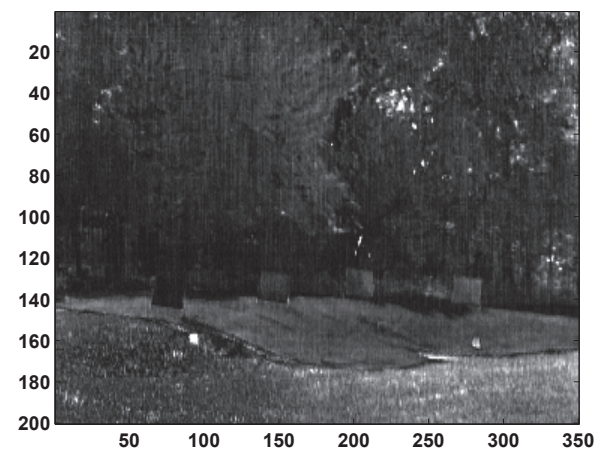

(a) No reflectance filtering

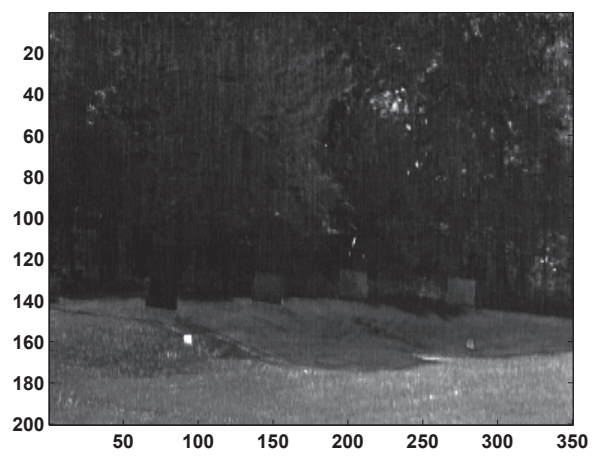

(b) Reflectance filtering

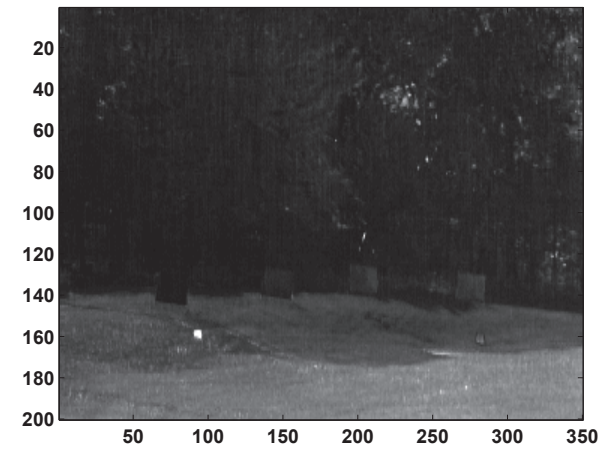

(c) Reflectance subspace

Figure 4.55: Comparison of detection statistic images for different MB reflectance modalities using $5 \%$ shadow initialization for AFRL data 


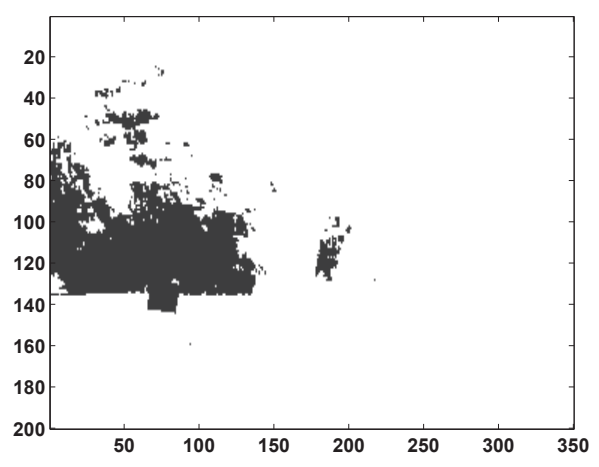

(a) $\tilde{\alpha}^{(1)}$

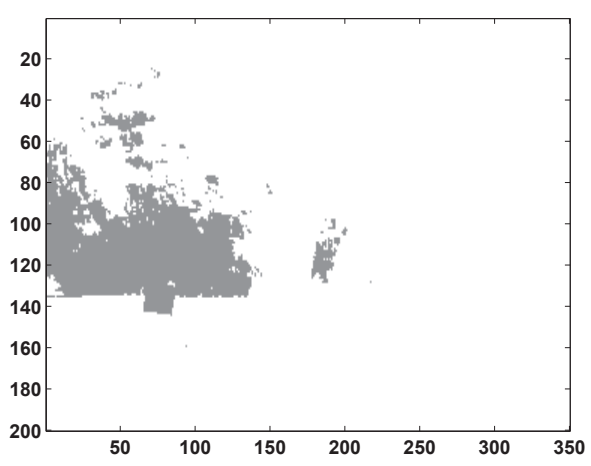

(b) $\beta^{(1)}$

Figure 4.56: Initial estimates for time-1 shadow coefficients using bottom $10 \%$ of spectral norm pixels for AFRL data

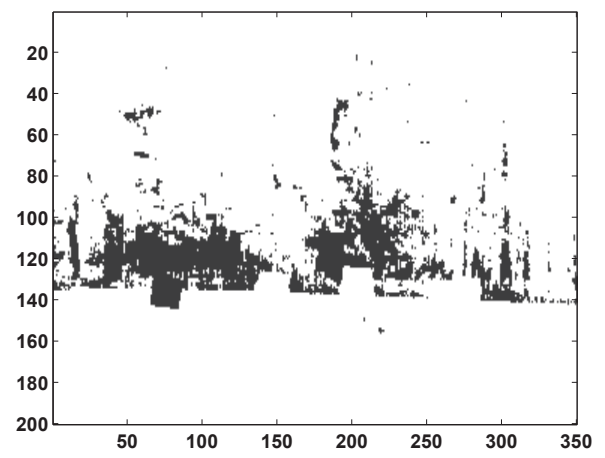

(a) $\tilde{\alpha}^{(2)}$

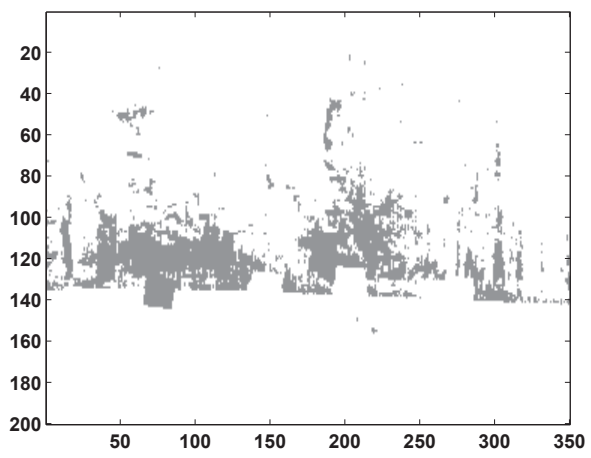

(b) $\beta^{(2)}$

Figure 4.57: Initial estimates for time-2 shadow coefficients using bottom $10 \%$ of spectral norm pixels for AFRL data 


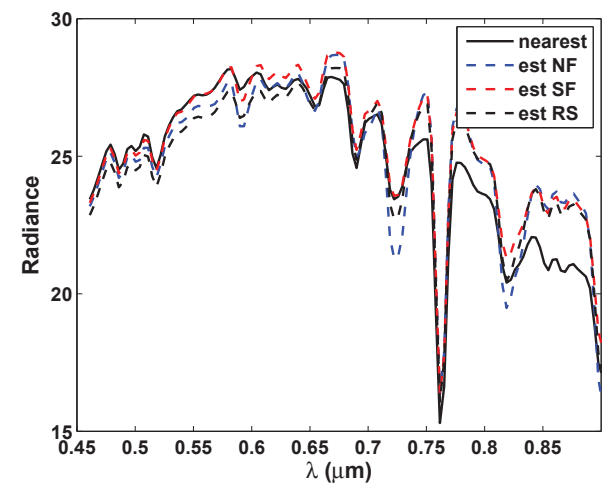

(a) $\boldsymbol{\tau} \odot \mathbf{L}_{s}$

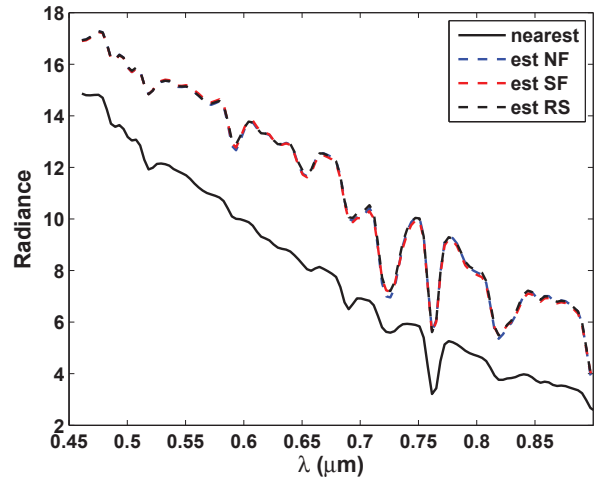

(b) $\boldsymbol{\tau} \odot \mathbf{L}_{d}$

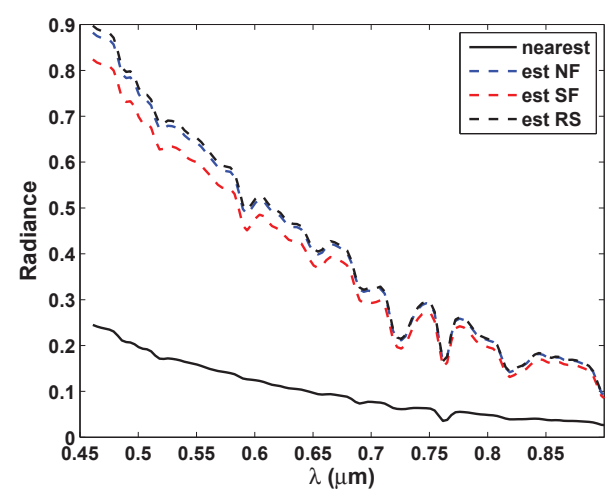

(c) $\mathbf{L}_{p}$

Figure 4.58: RT estimates for time-1 AFRL data for different reflectance estimation modalities using 10\% shadow initialization. 


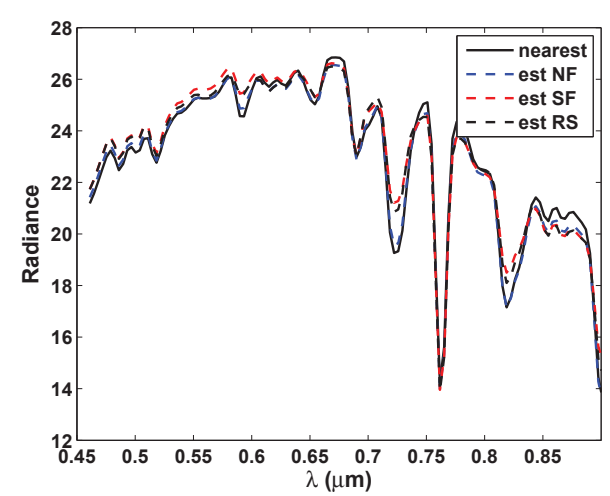

(a) $\boldsymbol{\tau} \odot \mathbf{L}_{s}$

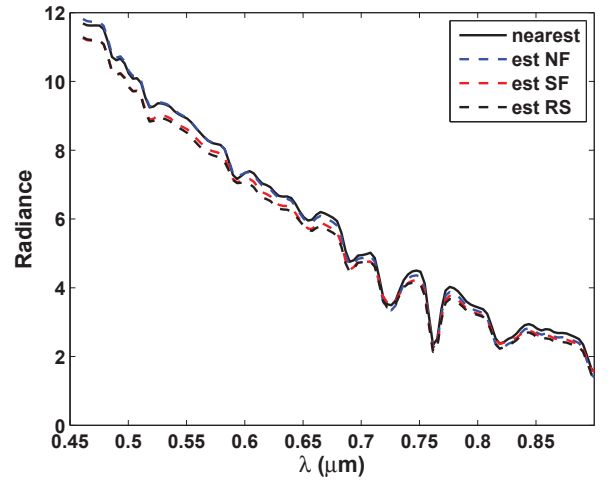

(b) $\boldsymbol{\tau} \odot \mathbf{L}_{d}$

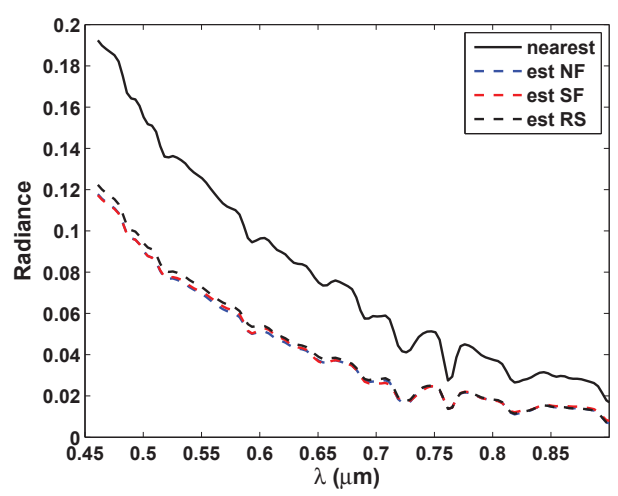

(c) $\mathbf{L}_{p}$

Figure 4.59: RT estimates for time-2 AFRL data for different reflectance estimation modalities using $10 \%$ shadow initialization. 
As previously, the shadow estimates using the various reflectance estimation modalities are all very similar. Consequently, only the results for the baseline non-filtering method are shown. Figures 4.60 and 4.61 display the shadow estimates for the time-1 and time-2 data, which are nearly identical to the previous case. The only significant

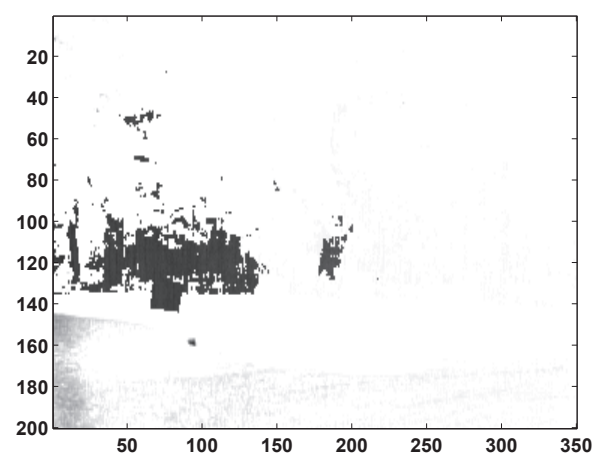

(a) $\hat{\tilde{\alpha}}^{(1)}$

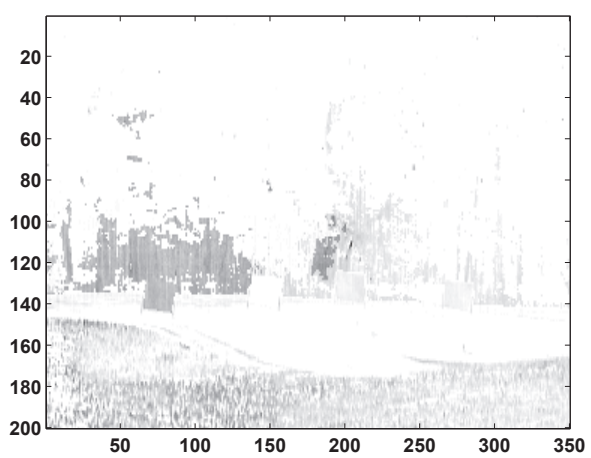

(b) $\hat{\beta}^{(1)}$

Figure 4.60: Estimated shadow coefficients for time-1 data using 10\% shadow initialization for AFRL data

differences occur for the additional $5 \%$ of pixels initialized as shadows. For very dark pixels, ambiguity exists between the shadow coefficients and the reflectance vectors estimated. Essentially, the same measurement can be represented using a low shadow coefficient or a low reflectance estimate, which is the cause of the difference in this case.

Figures 4.62 and 4.63 display the estimated measurement $\hat{L}$ for a grass pixel and a green panel pixel for both the time-1 and time-2 data. Additionally, Figure 4.64 displays the reflectance estimates associated with these pixels in comparison with laboratory truth. Again, the results are nearly identical to the previous shadow initial- 


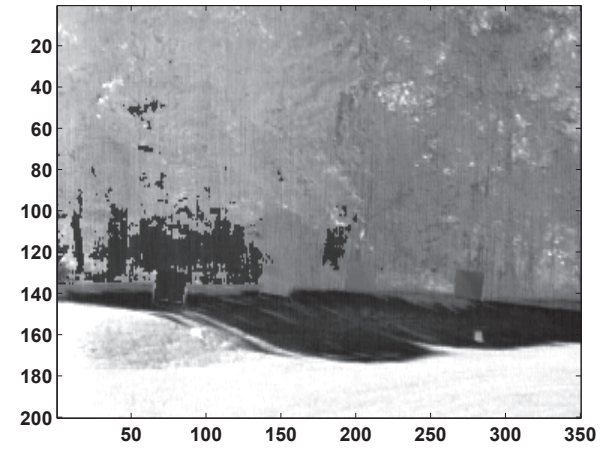

(a) $\hat{\tilde{\alpha}}^{(2)}$

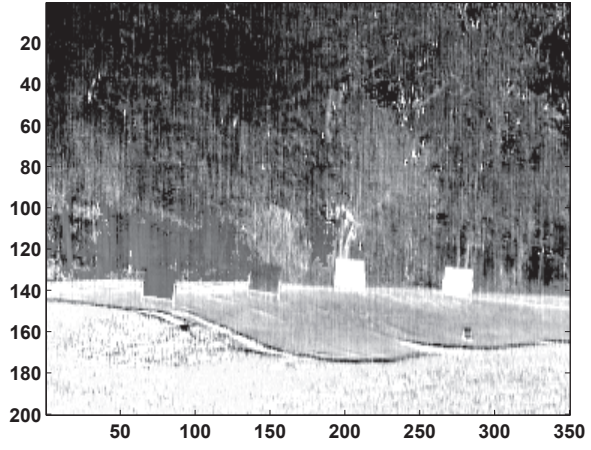

(b) $\hat{\beta}^{(2)}$

Figure 4.61: Estimated shadow coefficients for time-2 data using $10 \%$ shadow initialization for AFRL data

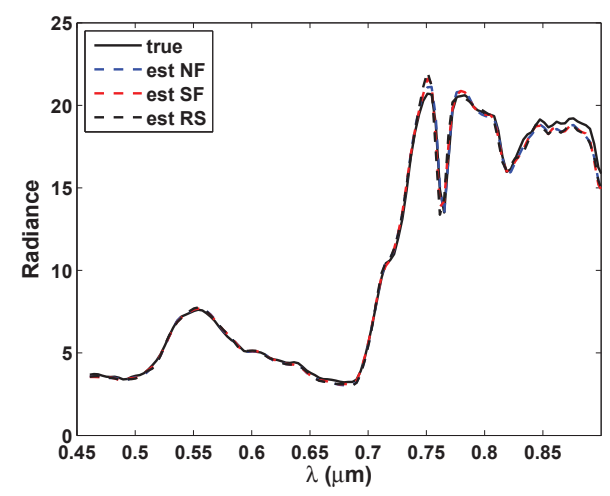

(a) Time-1

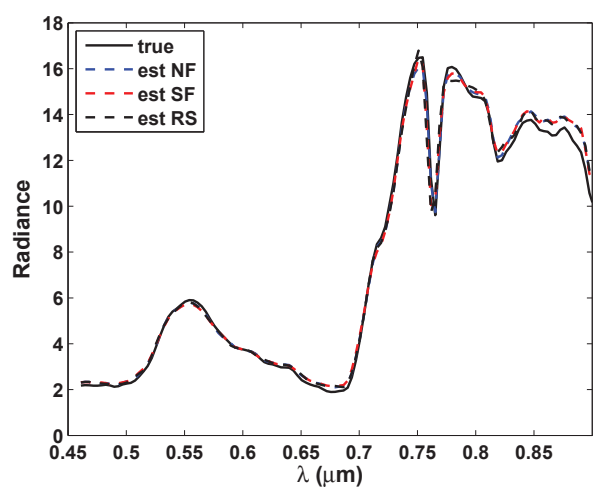

(b) Time-2

Figure 4.62: Comparison of estimated measurement $\hat{L}$ with true measurement $L$ for grass pixel using $10 \%$ shadow initialization for AFRL data 


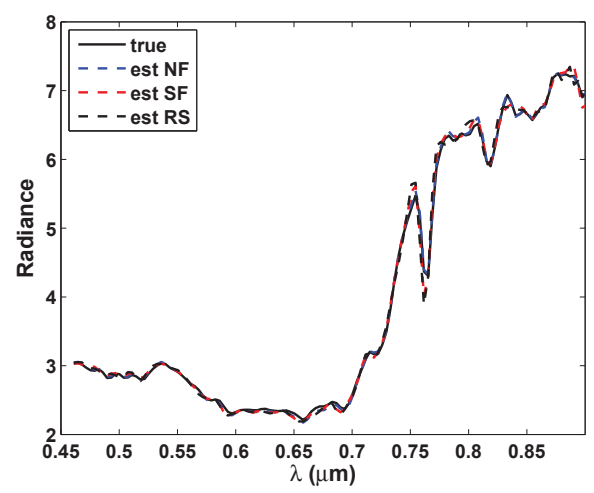

(a) Time-1

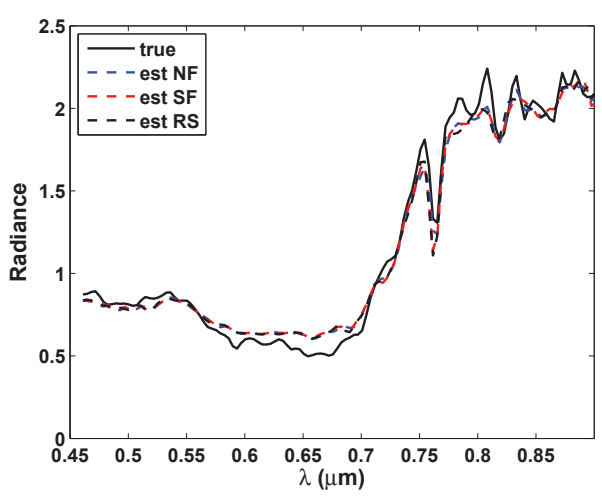

(b) Time-2

Figure 4.63: Comparison of estimated measurement $\hat{L}$ with true measurement $L$ for green panel pixel using 10\% shadow initialization for AFRL data

ization case. These results suggest a relative robustness to the shadow initialization used for this particular data set.

Figure 4.65 displays the empirical ROC curve performance using $10 \%$ shadow initialization in comparison with the performance of the $\mathrm{CC}$ and $\mathrm{CE}$ change detection algorithms discussed. As expected from the similarities of the state estimates, no noticeable difference in performance exists for this initialization case in comparison with the $5 \%$ shadow initialization case. Figure 4.66 displays the detection statistic images for the three reflectance modalities of the MB method, which reiterate this observation. Overall, the estimation and detection characteristics do not vary significantly when using a higher percentage of pixels for shadow initialization.

\section{SCR Results}

Examination of the SCR statistics provides further insight into any subtle differences in detection performance that may exist for the two initialization cases. Figures 


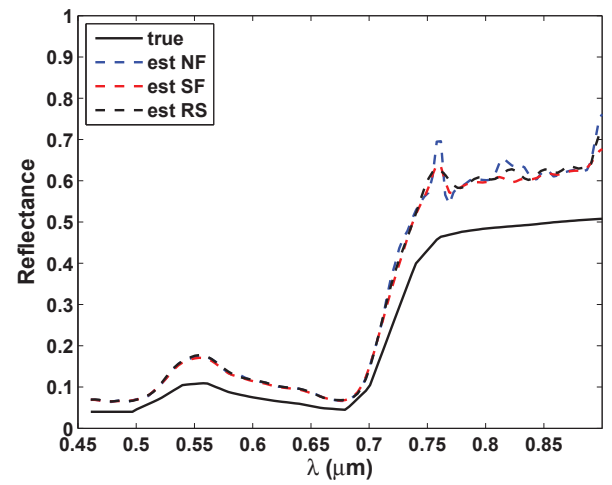

(a) Grass

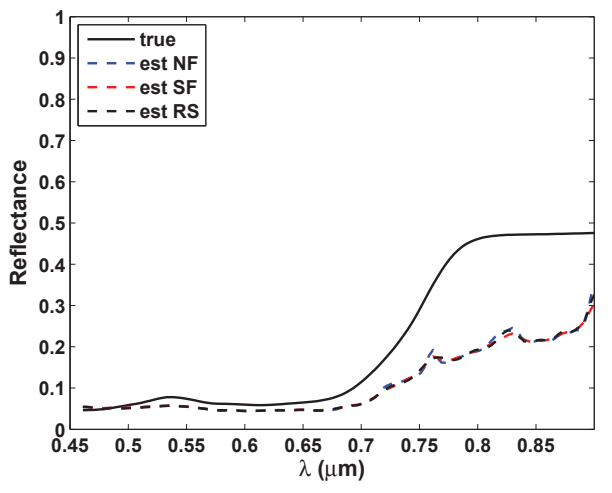

(b) Green panel

Figure 4.64: Comparison of estimated reflectance $\rho$ with true reflectance for grass pixel and green panel pixel using $10 \%$ shadow initialization for AFRL data

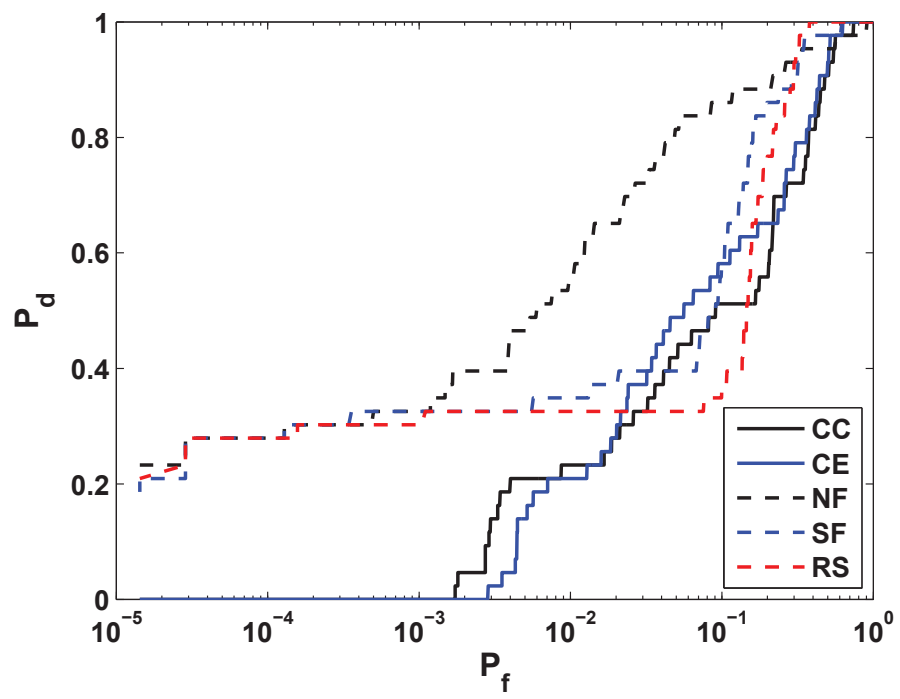

Figure 4.65: Empirical ROC curve performance for different reflectance estimation modalities using 10\% shadow initialization in comparison with $\mathrm{CC}$ and $\mathrm{CE}$ algorithms for AFRL data 


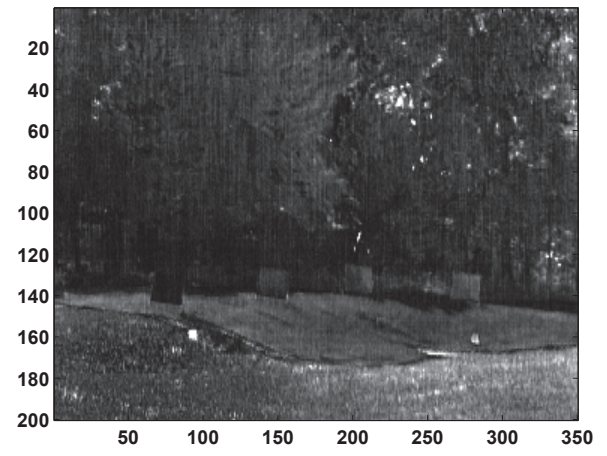

(a) No reflectance filtering

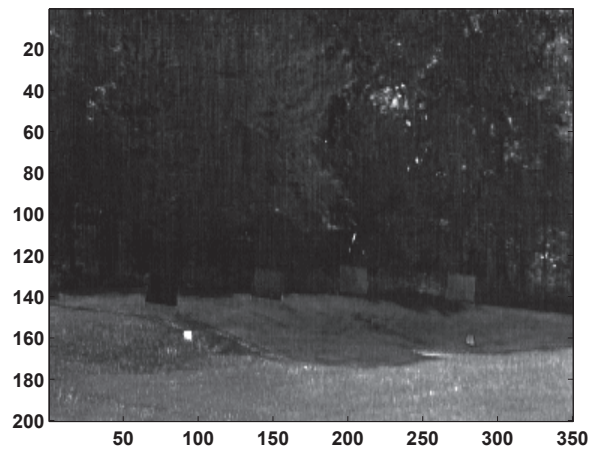

(b) Reflectance filtering

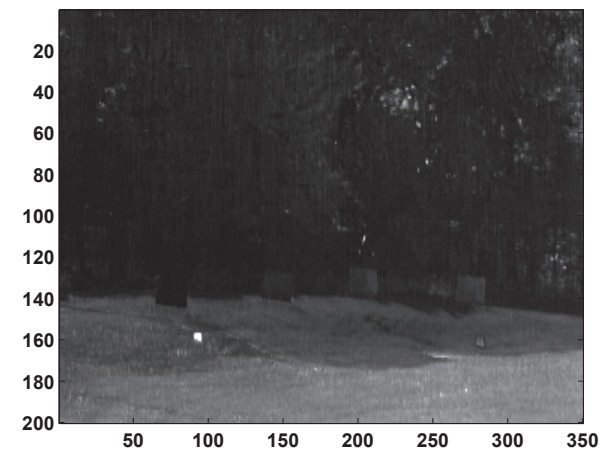

(c) Reflectance subspace

Figure 4.66: Comparison of detection statistic images for different MB reflectance modalities using $10 \%$ shadow initialization for AFRL data 
4.67 and 4.68 display the SCR for each target pixel in the scene for the different initialization cases. Figure 4.69 displays the SCR results for $\mathrm{CC}$ and $\mathrm{CE}$. Again, the

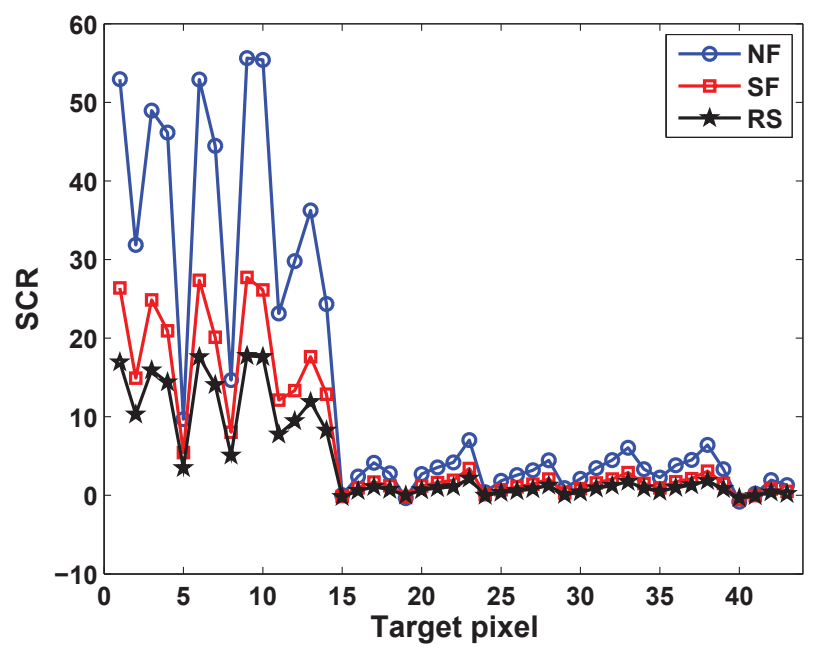

Figure 4.67: Comparison of SCR for different MB reflectance modalities using 5\% shadow initialization for AFRL data

SCR performance associated with the MB methods is much greater than the baseline $\mathrm{CC}$ and $\mathrm{CE}$ algorithms. The difference in SCR for the green tarp change and the more difficult camouflage tarp change is apparent as well. For this data set, negative SCR values result for several of the change targets when the detector output for a target pixel is less than the mean detector output for the background. The average SCR results are provided in Table 4.4. In general, the MB methods achieve a higher SCR than the CC and CE methods. Amongst the MB methods, the non-filtering (NF) method achieves the highest SCR. Minimal difference exists in SCR for the different shadow initializations. Other interesting observations can be made with regard to the background statistics. From earlier theoretical analysis, the expected 


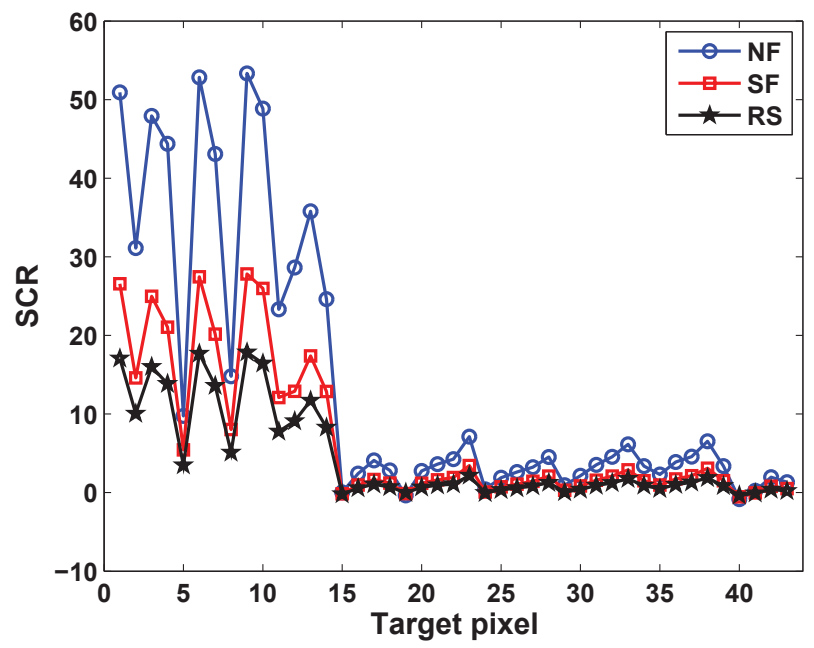

Figure 4.68: Comparison of SCR for different MB reflectance modalities using 10\% shadow initialization for AFRL data

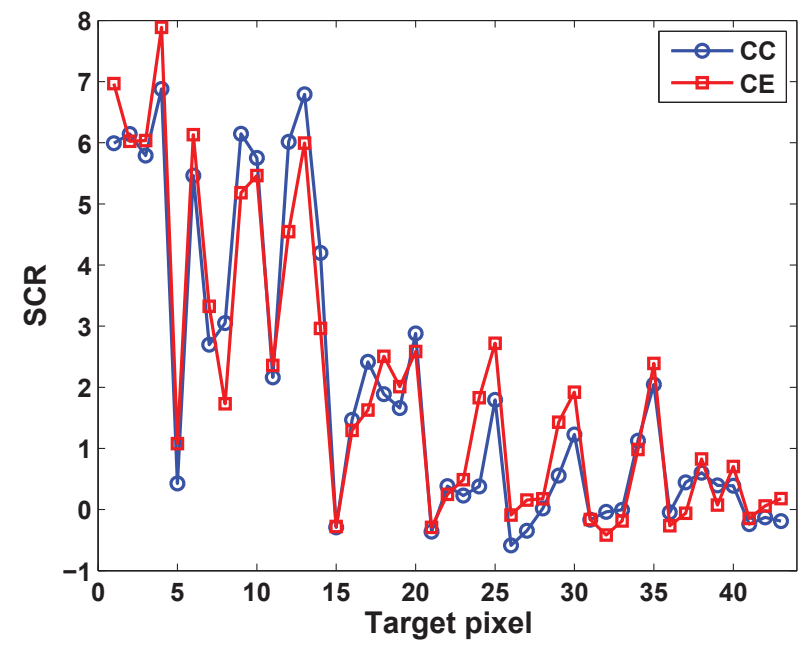

Figure 4.69: Comparison of SCR for CC and CE algorithms for AFRL data 


\begin{tabular}{|c|c|c|c|c|c|}
\hline Algorithm & $\hat{\mu}_{t}$ & $\hat{\mu}_{b}$ & $\hat{\mu}_{t}-\hat{\mu}_{b}$ & $\hat{\sigma}_{b}$ & $\hat{\mu}_{S C R}$ \\
\hline CC & 27.4908 & 9.9891 & 17.5017 & 8.8528 & 1.9770 \\
\hline CE & 29.5145 & 9.9879 & 19.5267 & 9.5423 & 2.0463 \\
\hline NF-5\% & 7462.0 & 592.6055 & 6870.0 & 485.5563 & 14.1491 \\
\hline NF-10\% & 7172.0 & 587.242 & 6585.0 & 477.63 & 13.7879 \\
\hline SF-5\% & 7468.1 & 994.9648 & 6473.2 & 946.1968 & 6.8412 \\
\hline SF-10\% & 7415. & 990.6733 & 6425.0 & 939.8598 & 6.8357 \\
\hline RS-5\% & 7701.0 & 1356.0 & 6345.0 & $1.426 \mathrm{e} 3$ & 4.449 \\
\hline RS-10\% & 7576.0 & 1348.0 & 6228.0 & $1.418 \mathrm{e} 3$ & 4.3931 \\
\hline
\end{tabular}

Table 4.4: Signal to clutter ratio for various algorithms using AFRL data set

mean and variance of the background are $2 K$ and $4 K$ respectively. For this data set, $K=121$. Correspondingly, one expects $\mu_{b}=242$ and $\sigma_{b}^{2}=484$. However, the sample mean and variance of the MB methods are much greater than the expected values, signifying either model mismatch or poor estimation performance. Without truth information about the data, it is difficult to determine the exact cause. Even with larger than expected residual optimization error, the MB methods achieve superior detection performance than $\mathrm{CC}$ and $\mathrm{CE}$. This result suggests that the model mismatch error is smaller than the residual optimization error that results when the null hypothesis is violated. If the residual optimization error results from model mismatch, these detection results suggest that even without a perfect data model, good detection performance can be achieved.

\section{Impact of White Noise Model}

The signal-dependent noise model has been applied for the MB change detection tests on the AFRL data because the noise stats have been estimated with a high degree of confidence. As a result, the impact of applying a signal-dependent noise 
model versus a simple white noise model can explicitly be tested for these data. The MB change detection algorithm using 5\% shadow initialization and no spectral filtering for reflectance is applied here using a white noise model rather than the signal-dependent model. Figure 4.70 displays the SCR and ROC performance using the white noise model as compared with the signal-dependent model. Both the SCR

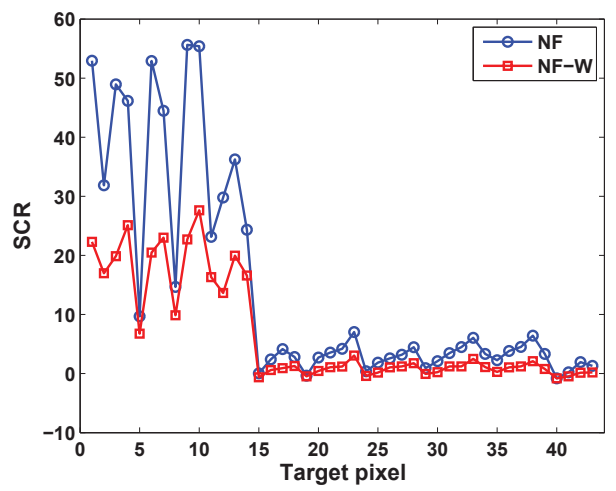

(a) SCR

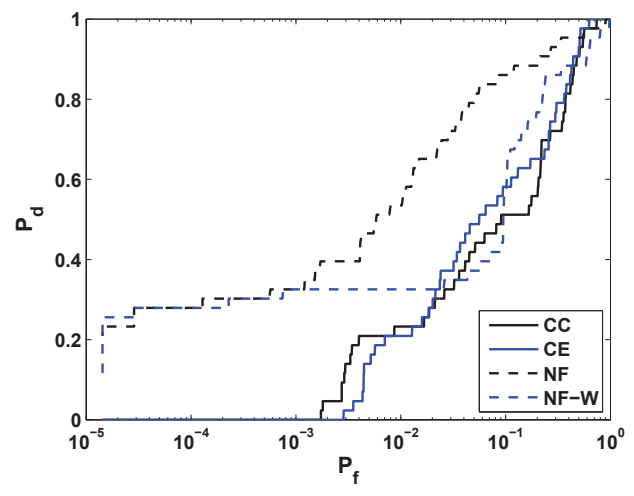

(b) ROC

Figure 4.70: Comparison of detection performance using a signal-dependent noise model (NF) versus a white noise model (NF-W) for AFRL data

and ROC performance improve using the signal-dependent noise model. The average SCR using the white noise model is 6.58 whereas that using the signal-dependent noise model is 14.15. Figure 4.71 displays the detection statistic images associated with both cases. The camouflage target to the right blends into the clutter more for the white noise model than the signal-dependent model. This result is reasonable when considering the nature of the signal-dependent noise model. The camouflage target has very low reflectance and the target location resides in the shade at time- 2 . As a result, the measured signal for both time-1 and time- 2 for the associated target pixels 


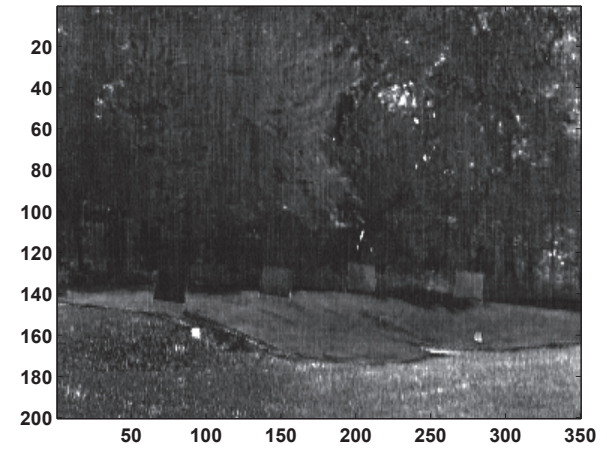

(a) Signal-dependent noise

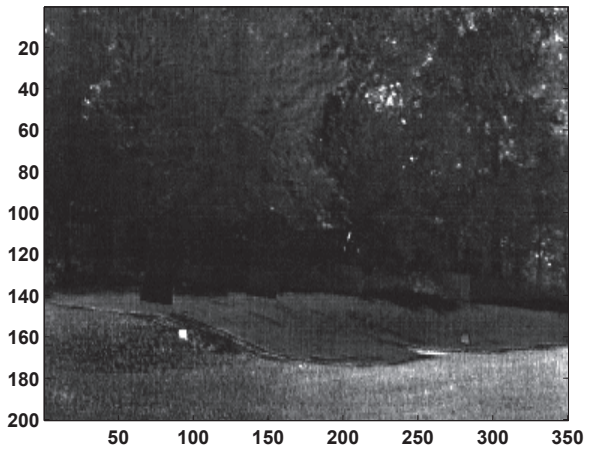

(b) White noise

Figure 4.71: Comparison of detection statistic images using a signal-dependent noise model versus a white noise model for AFRL data

has a relatively low magnitude in comparison with the other measurements of the scene. For the signal-dependent noise model, the noise variance for these pixels will be lower than for other brighter pixels. The residual optimization error is weighted by the inverse covariance matrix. As a result, the residual error for the camouflage target pixels is weighted more heavily than for the white noise model for which the residual error for all pixels is weighted equally.

The estimation results are examined as well in order to determine the impact of the noise model on various state estimates. Figures 4.72 and 4.73 display the RT estimates for time-1 and time-2. The RT estimates for time-1 are very similar for both noise models. However, the RT estimates for the time-2 data differ significantly for the two noise models. The reason for this difference is not entirely clear at this point. Figures 4.74 and 4.75 display the estimated shadow coefficients for time- 1 and time-2 using the white noise model. These estimates are similar to those obtained using the signal-dependent noise model as shown in Figures 4.48 and 4.49. 


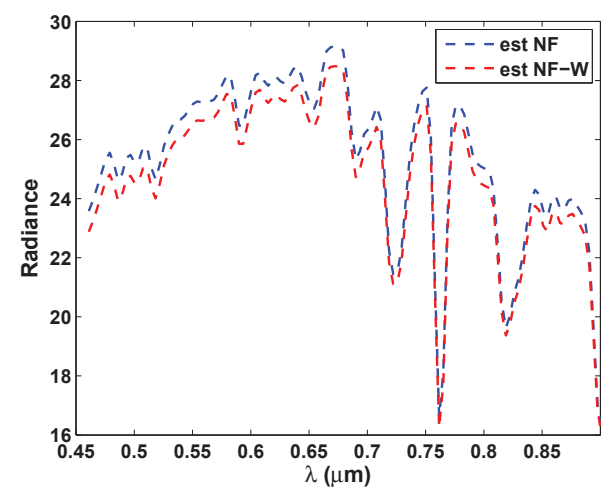

(a) $\boldsymbol{\tau} \odot \mathbf{L}_{s}$

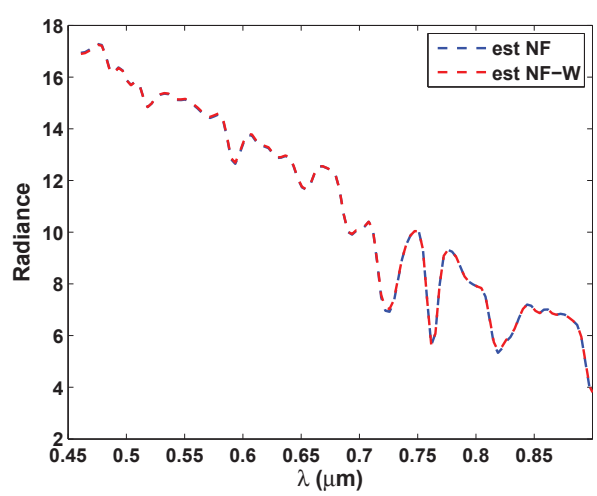

(b) $\boldsymbol{\tau} \odot \mathbf{L}_{d}$

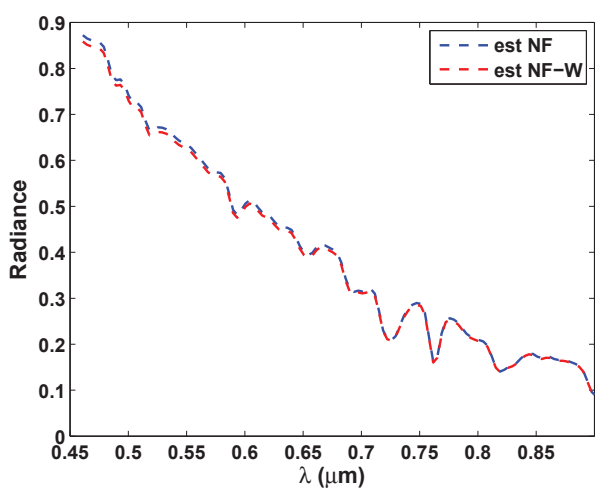

(c) $\mathbf{L}_{p}$

Figure 4.72: RT estimates for time-1 AFRL data using a signal-dependent noise model $(\mathrm{NF})$ versus a white noise model (NF-W) 


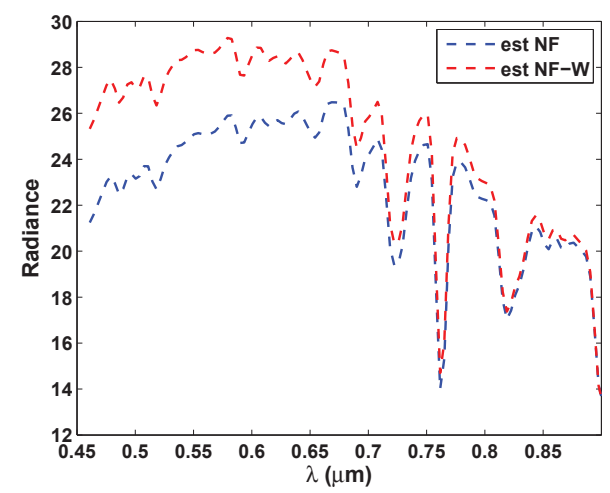

(a) $\boldsymbol{\tau} \odot \mathbf{L}_{s}$

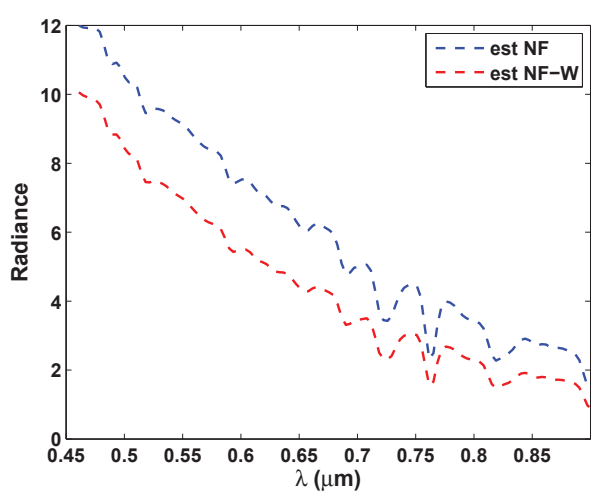

(b) $\boldsymbol{\tau} \odot \mathbf{L}_{d}$

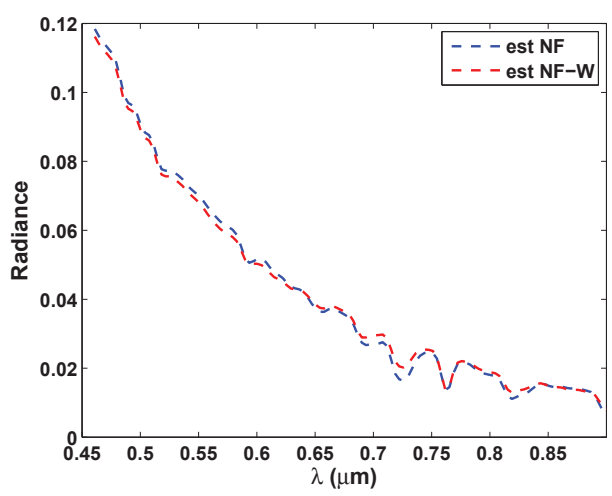

(c) $\mathbf{L}_{p}$

Figure 4.73: RT estimates for time-2 AFRL data using a signal-dependent noise model $(\mathrm{NF})$ versus a white noise model $(\mathrm{NF}-\mathrm{W})$ 


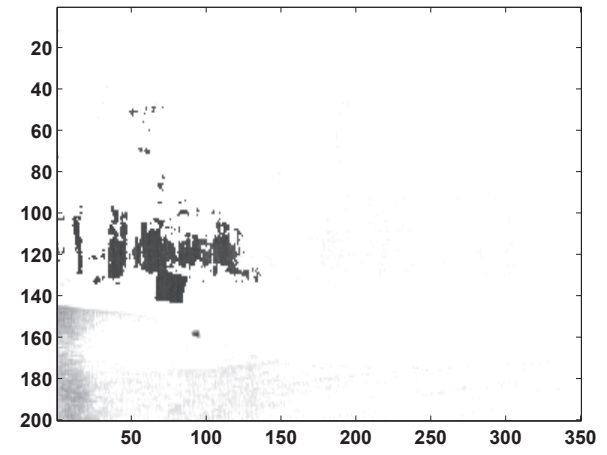

(a) $\hat{\tilde{\alpha}}^{(1)}$

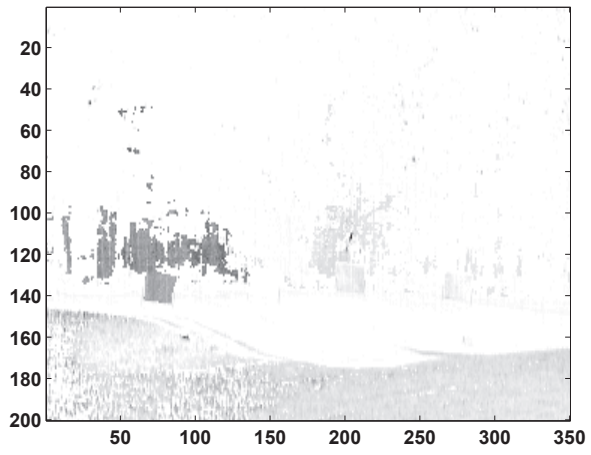

(b) $\hat{\beta}^{(1)}$

Figure 4.74: Estimated shadow coefficients for time-1 data using white noise model for AFRL data

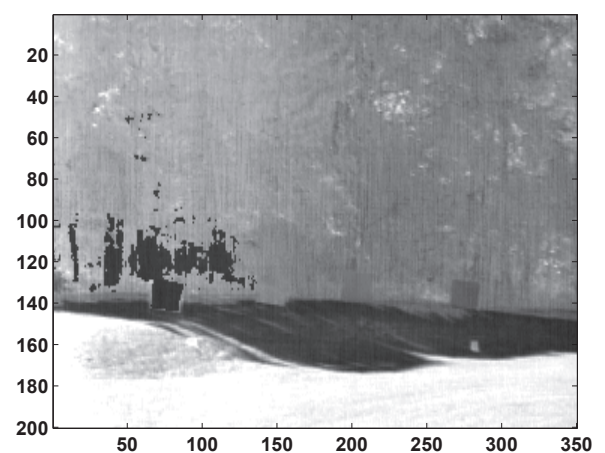

(a) $\hat{\tilde{\alpha}}^{(2)}$

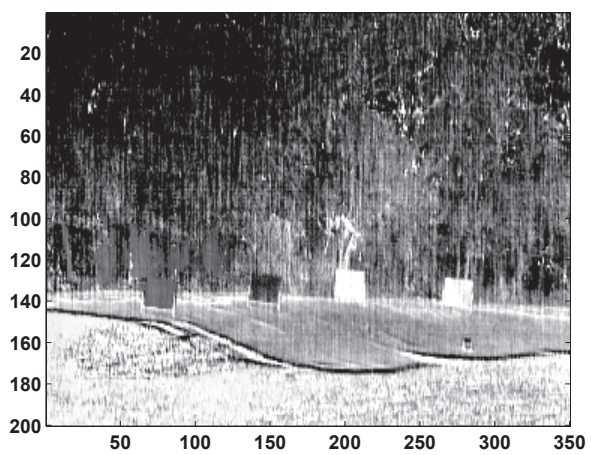

(b) $\hat{\beta}^{(2)}$

Figure 4.75: Estimated shadow coefficients for time-2 data using white noise model for AFRL data 


\subsubsection{Results using Relatively Calibrated Data Model}

The AFRL data are calibrated to absolute sensor-reaching radiance units, which allows for the use of the original data model given in (2.2). However, the relatively calibrated data model given in (2.84) may provide an avenue for compensation of residual calibration error. To test this concept, the relatively calibrated data model is applied with the 4-step AO algorithm on the absolutely calibrated AFRL data. Since the data has been calibrated to an absolute level, the relative gain and offset terms are expected to have values of $\mathbf{a}_{g}^{(t)}[m]=1$ and $\mathbf{b}_{g}^{(t)}[m]=0$. As such, these values are used as the initial estimates for the optimization algorithm. The baseline optimization algorithm with no spectral filtering on reflectance is applied for 20 iterations and $I=10$ as in the previous cases. Additionally, the shadow and basis coefficients are initialized using the bottom $5 \%$ of spectral norm pixels as before.

Figures 4.76 and 4.77 display the final estimated gain and offset terms associated with the relatively calibrated data model. As discussed, one would expect the gain

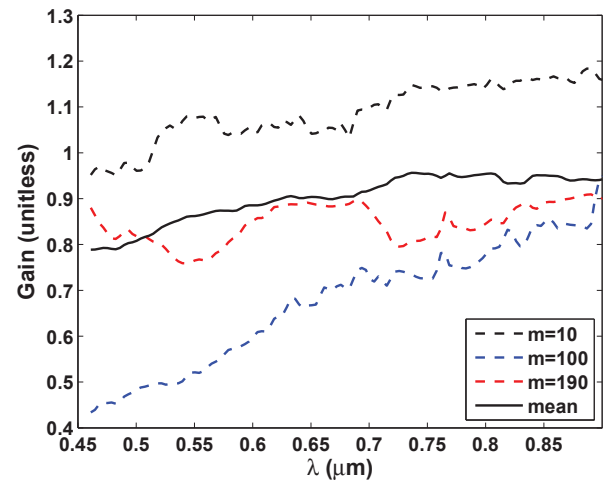

(a) Time-1

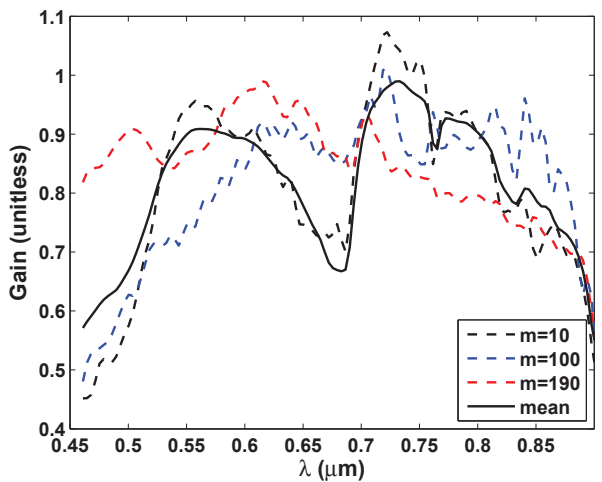

(b) Time-2

Figure 4.76: Estimated relative gain $\mathbf{a}_{g}^{(t)}$ using relatively calibrated data model on calibrated AFRL data 


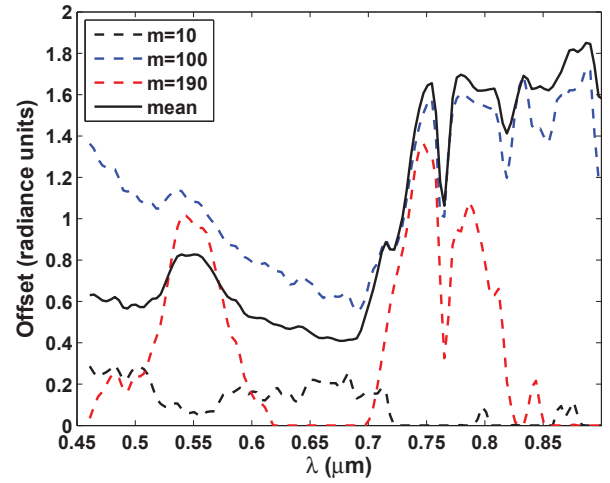

(a) Time-1

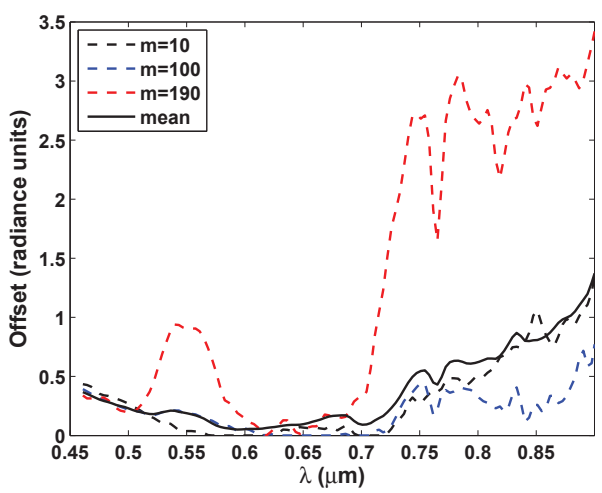

(b) Time-2

Figure 4.77: Estimated relative offset $\mathbf{b}_{g}^{(t)}$ using relatively calibrated data model on calibrated AFRL data

term to be at or near 1 for all bands, assuming good calibration, an accurate data model, and accurate model parameter estimates. Similarly, the offset is expected to be at or near 0 for all bands. For the time-1 data, the gain and offset associated with rows near the top of the scene $(m=10)$ are close to the expected values. However, as the row position gets larger, the gain and offset vary more significantly. The gain and offset for the time-2 data demonstrate much larger variability and spectral structure than those for the time-1 data. The exact cause for this is unclear. The estimated values may be compensating for residual calibration error or model-mismatch error. The estimated offset terms display a spectral structure similar to vegetation spectra, which may suggest that the offset is compensating for adjacency effects produced by the treeline, which are not described by the original data model. 
Figures 4.78 and 4.79 display the estimated shadow coefficients using the relative calibration model and 4-step AO algorithm. The estimates are similar to those obtained using the original data model and correlate well with the shadow conditions of the scenes.

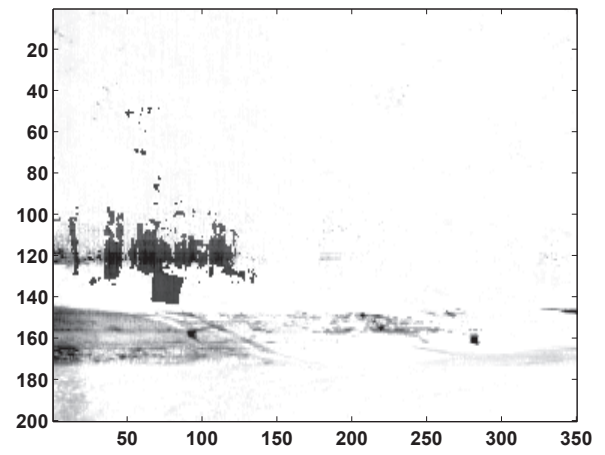

(a) $\hat{\tilde{\alpha}}^{(1)}$

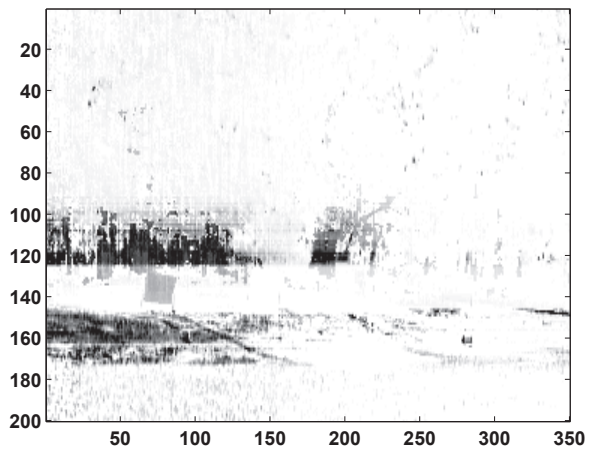

(b) $\hat{\beta}^{(1)}$

Figure 4.78: Estimated shadow coefficients for time-1 data using 5\% shadow initialization with relatively calibrated data model for AFRL data

\subsubsection{Results using Uncalibrated Data Model}

The algorithm is additionally applied to the raw, uncalibrated AFRL imagery using the uncalibrated data model of (2.80). Application on the uncalibrated imagery may be valuable if significant calibration error exists in the calibrated imagery. In this case, the noise model must be adjusted appropriately as the noise statistics of the uncalibrated data are different than those of the calibrated data. The total noise 


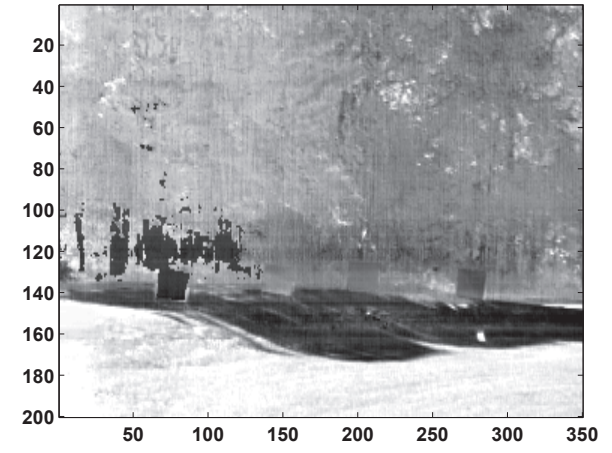

(a) $\hat{\tilde{\alpha}}^{(2)}$

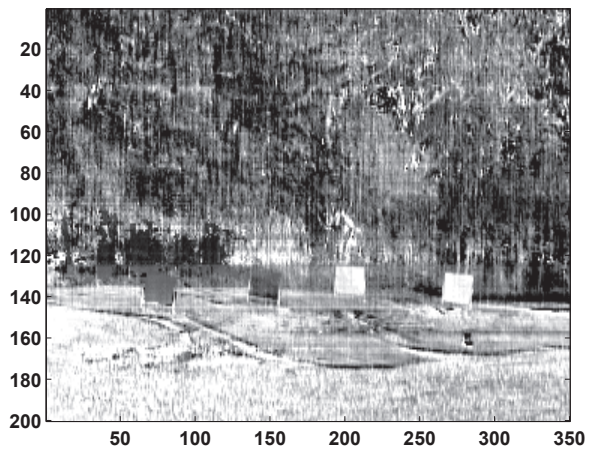

(b) $\hat{\beta}^{(2)}$

Figure 4.79: Estimated shadow coefficients for time-2 data using 5\% shadow initialization with relatively calibrated data model for AFRL data

variance for the uncalibrated imagery as examined in Chapter 3 is given by,

$$
\begin{aligned}
\sigma_{R}^{2}[m, n, k] & =\sigma_{s}^{2}[m, n, k]+\sigma_{i}^{2}[m, k] \\
& =g[m, k]\left(\mu_{R}[m, n, k]-b[m, k]\right)+\sigma_{i}^{2}[m, k],
\end{aligned}
$$

where $\mu_{R}[m, n, k]$ is the mean signal. For this work, the current estimate of the dark current $\hat{b}^{(t)}[m, k]$ is used to obtain an estimate of the noise variance. From the previous noise analysis, the estimated model parameters are,

$$
\hat{g}[m, k]=\hat{g}=0.09 D N / e^{-}
$$

and

$$
\hat{\sigma}_{i}^{2}[m, k]=\hat{\sigma}_{i}^{2}[k]=\left\{\begin{array}{ll}
15.9 D N^{2} & \lambda_{k} \leq 0.72 \mu m \\
11.3 D N^{2} & \lambda_{k}>0.72 \mu m
\end{array} .\right.
$$

While the data is uncalibrated, it is spectrally filtered to improve SNR. Consequently, the noise stats are altered by,

$$
\sigma_{R_{f}}^{2}[m, n, k] \approx P_{f} \sigma_{R}^{2}[m, n, k]
$$


which results in,

$$
\sigma_{R_{f}}^{2}[m, n, k] \approx \frac{P_{f} g}{S_{f}}\left(\mu_{R}[m, n, k]-b[m, k]\right)+P_{f} \sigma_{i}^{2}[k]
$$

Using the measurement itself as an approximation of the mean signal, the noise variance is estimated using,

$$
\left(\sigma_{R_{f}}^{(t)}\right)^{2}[m, n, k] \approx \frac{P_{f} g}{S_{f}}\left(r_{f}^{(t)}[m, n, k]-\hat{b}^{(t)}[m, k]\right)+P_{f} \sigma_{i}^{2}[k]
$$

where $r_{f}$ is the uncalibrated, spectrally-filtered measurement.

For the uncalibrated imagery, an initial estimate for the effective sensor gain coefficient $\mathbf{a}^{(t)}$ and the dark current offset $\mathbf{b}^{(t)}$ are required. Two cases are examined here. First, the gain and dark current offset obtained from the calibration data are used as the initial estimates. In this case, one would expect nearly equivalent results as in the previous case where the calibrated data is used with the relatively calibrated data model. In the second case, the gain and dark current are initialized assuming no prior knowledge about these quantities. In this case, the quantities are estimated using the NDVI method described in Section 2.6.

\section{Using Calibration Data for Initial Estimates of Detector Gain and Dark Current}

The results using the calibration data as initial estimates are examined first. Figures 4.80 and 4.81 display the estimated sensor gain terms in comparison with the gain terms used for initialization obtained from the calibration data. The gain estimates for the time-1 data have a similar spectral shape to those used to calibrate the imagery but vary in magnitude, whereas the time-2 estimated gain possesses additional spectral structure. Figures 4.82 and 4.83 display the estimated dark current offset terms in comparison with those obtained from the calibration data. Again, 


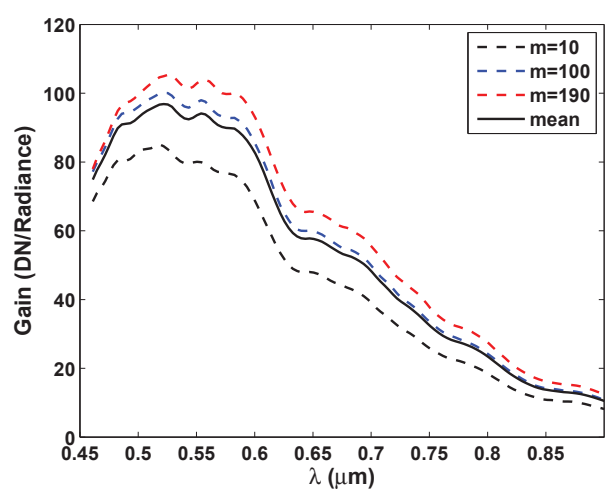

(a) Initialization from calibration data

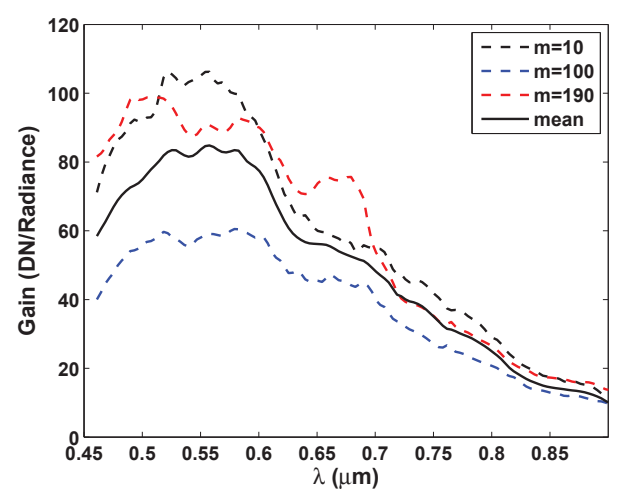

(b) Estimate

Figure 4.80: Estimate of time-1 effective sensor gain coefficient $\mathbf{a}^{(1)}$ in comparison with calibration data for AFRL data

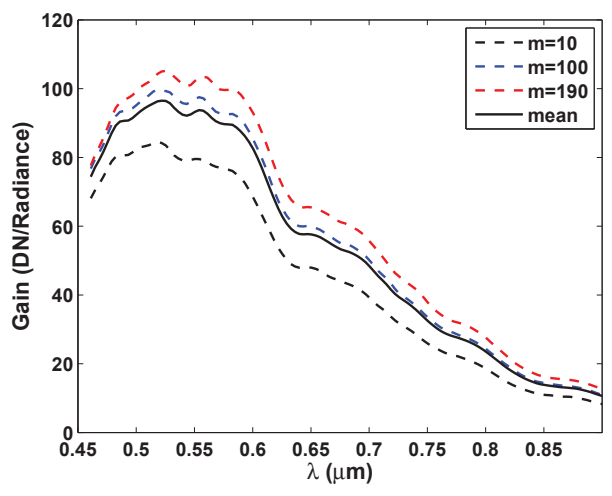

(a) Initialization from calibration data

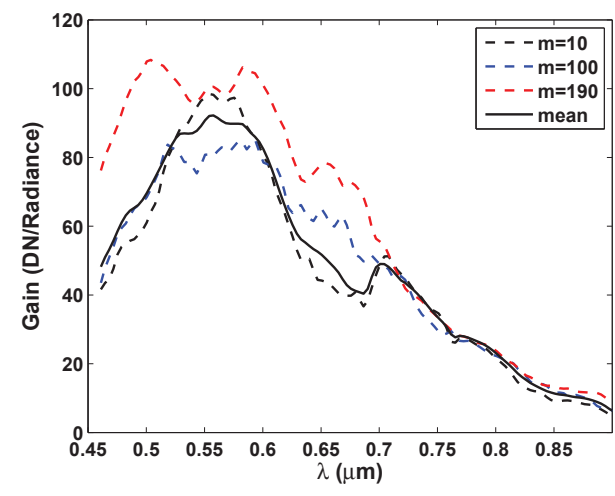

(b) Estimate

Figure 4.81: Estimate of time-2 effective sensor gain coefficient $\mathbf{a}^{(2)}$ in comparison with calibration data for AFRL data 
the estimated quantities demonstrate spectral structure different than that expected based upon the calibration data. One can note that the sensor gain and dark cur-

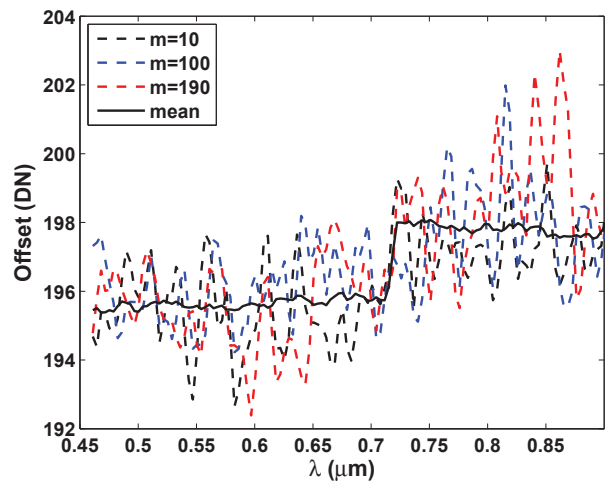

(a) Initialization from calibration data

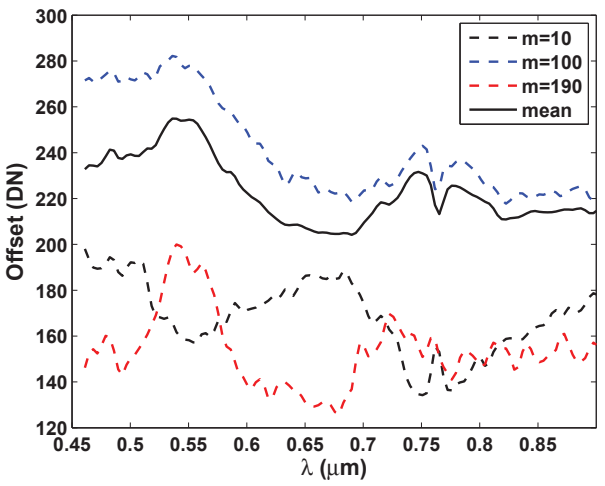

(b) Estimate

Figure 4.82: Estimate of time-1 dark current offset $\mathbf{b}^{(1)}$ in comparison with calibration data for AFRL data

rent offset used to calibrate the time- 1 and time- 2 data are nearly identical. This result suggests that differences present in the final estimated quantities most likely do not result from residual calibration error as one would expect both estimates to demonstrate similar irregularities. Consequently, the estimated gain and offset may be compensating for adjacency effects not accounted for in the data model. The significance of the adjacency effects can vary between data sets depending on the solar position and the turbulence of the atmosphere.

Figures 4.84 and 4.85 display the estimated shadow coefficients for this case. The estimates remain similar to those obtained for the previous case using the absolutely calibrated data. 


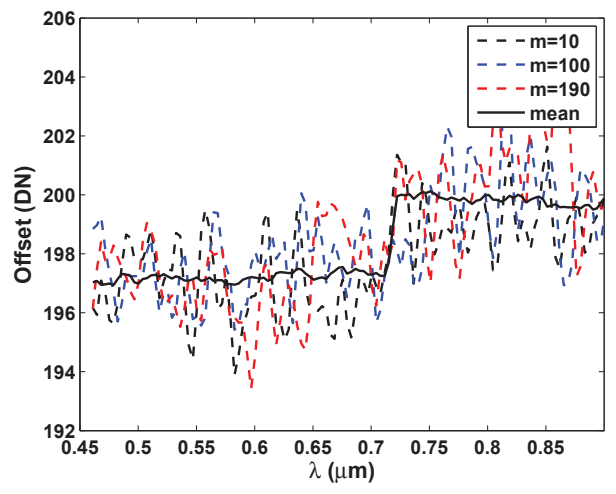

(a) Initialization from calibration data

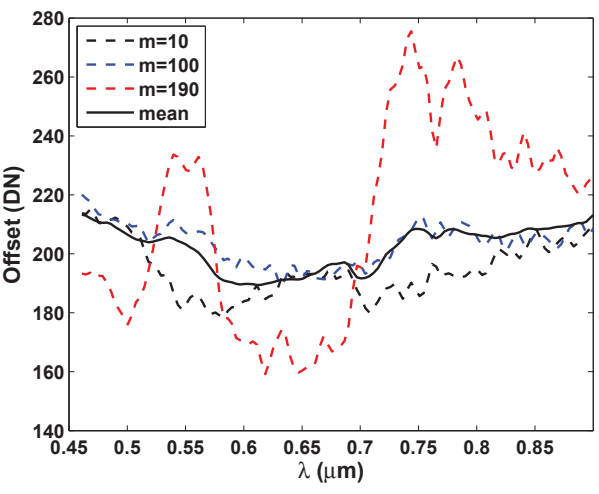

(b) Estimate

Figure 4.83: Estimate of time-2 dark current offset $\mathbf{b}^{(2)}$ in comparison with calibration data for AFRL data

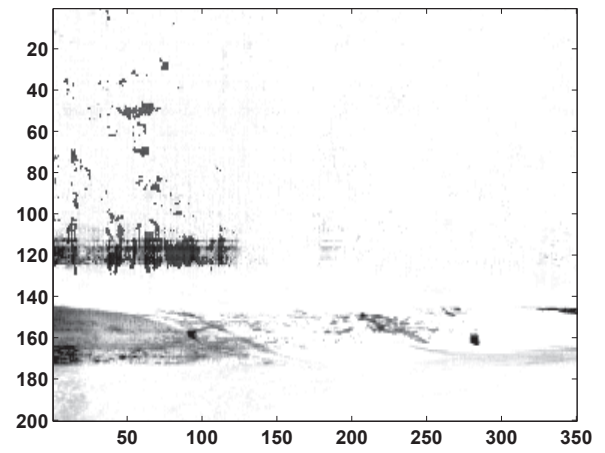

(a) $\hat{\tilde{\alpha}}^{(1)}$

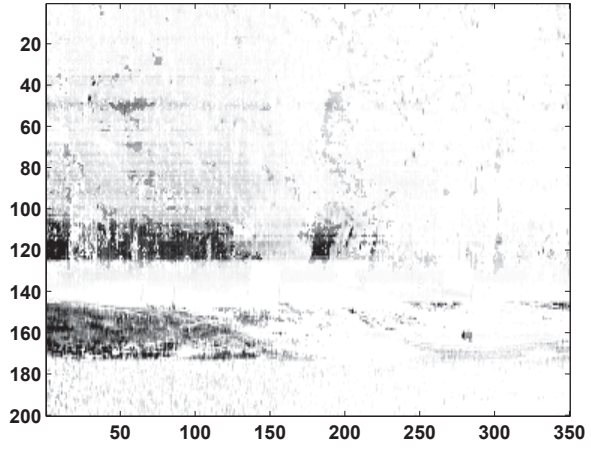

(b) $\hat{\beta}^{(1)}$

Figure 4.84: Estimated shadow coefficients for time-1 data using 5\% shadow initialization with uncalibrated data model for AFRL data 


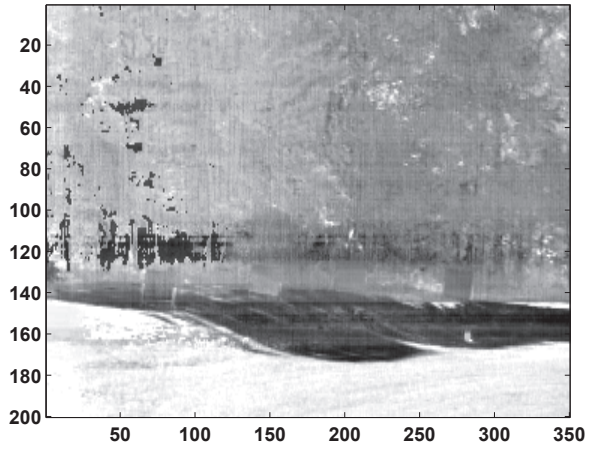

(a) $\hat{\tilde{\alpha}}^{(2)}$

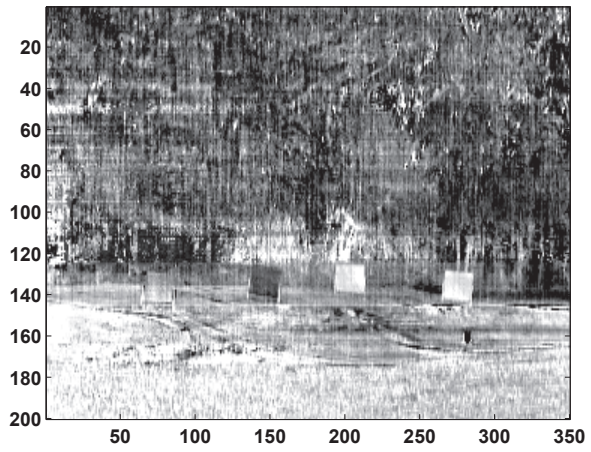

(b) $\hat{\beta}^{(2)}$

Figure 4.85: Estimated shadow coefficients for time-2 data using 5\% shadow initialization with uncalibrated data model for AFRL data

Figure 4.86 displays the estimated reflectance for a grass pixel for the MB methods using the original data model (NF), the relatively calibrated data model (NF-G), and the uncalibrated data model (NF-R). The estimates for all methods are fairly similar.

Figure 4.87 compares the ROC performance for these MB methods as well. The detection performance improves at higher false alarm rates when using the uncalibrated and relatively calibrated data models. This may result from improved suppression of model mismatch error resulting from adjacency from the treeline in the background, which appears in the estimated dark current offset. However, detection performance decreases at lower false alarm rates. The average SCR of the three methods are $S C R_{N F}=14.15, S C R_{N F-G}=14.14$, and $S C R_{N F-R}=18.67$. Figure 4.88 displays the detection statistic images of the three model-based methods to obtain further insight into improved clutter suppression. The residual clutter in the grass region has been reduced for both the uncalibrated and relatively calibrated data model cases in comparison with the results from the absolutely calibrated data. However, new 


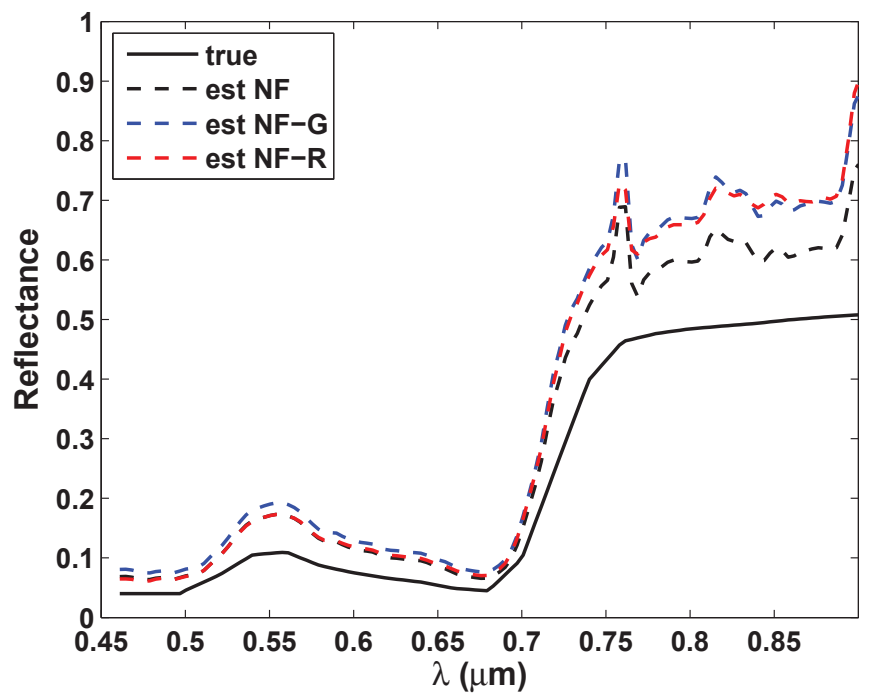

Figure 4.86: Comparison of estimated reflectance $\rho$ with library reflectance for the MB methods using the original data model (NF), the relatively calibrated data model (NF-G), and the uncalibrated data model (NF-R) for AFRL data

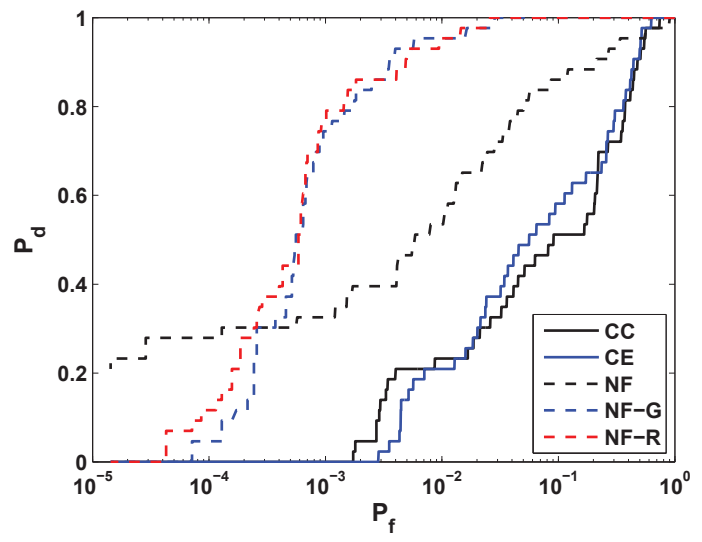

Figure 4.87: Empirical ROC curve performance for the MB methods using the original data model (NF), the relatively calibrated data model (NF-G), and the uncalibrated data model (NF-R) in comparison with $\mathrm{CC}$ and $\mathrm{CE}$ algorithms for AFRL data 


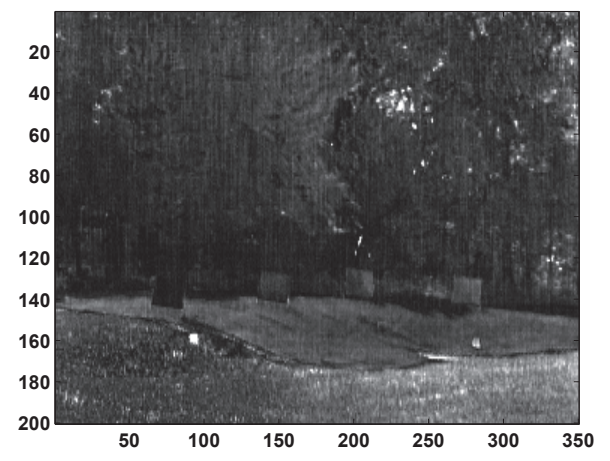

(a) Original data model

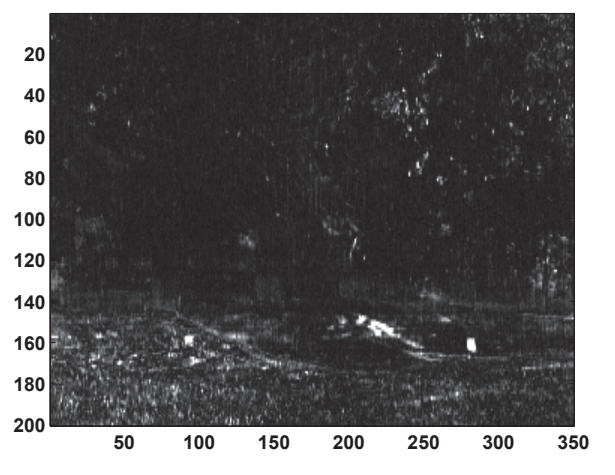

(b) Relative calibration data model

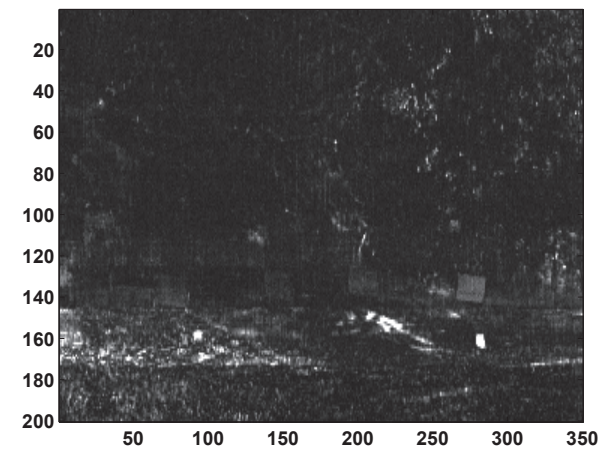

(c) Raw data model

Figure 4.88: Comparison of detection statistic images for MB methods using the original data model, the relatively calibrated data model, and the uncalibrated data model for AFRL data 
residual clutter regions exist in the detection statistic image for the uncalibrated and relatively calibrated data model results that are not found in the absolutely calibrated results. These residual clutter regions are associated with a dirt patch in the grass region and several other areas in the grass, which produce the decline in performance at lower false alarms. The exact reason for this additional clutter is unknown at this time but may be related to small natural reflectance changes or specular reflectance characteristics for the materials in these pixels.

\section{Using NDVI to Obtain Initial Estimates of Detector Gain and Dark Cur- rent}

Next, the NDVI method is used to obtain initial estimates of the detector gain and dark current offset for the uncalibrated data model. Figures 4.89 and 4.90 display the initial estimates of the gain and offset obtained using the NDVI method in comparison with the mean gain and offset obtained from the calibration data used for initialization in the previous section. The estimated gain possesses the same general shape

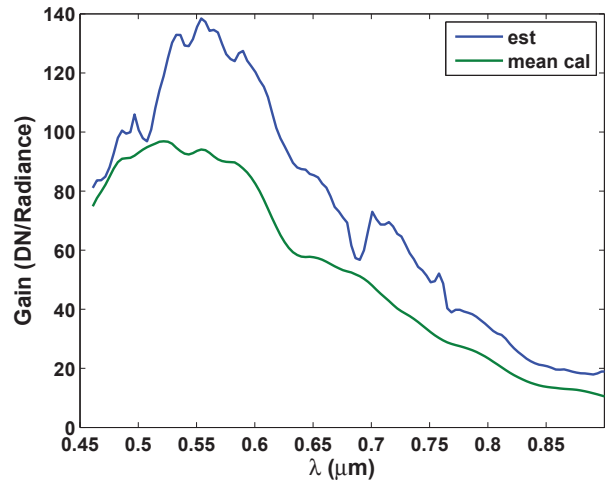

(a) Time-1

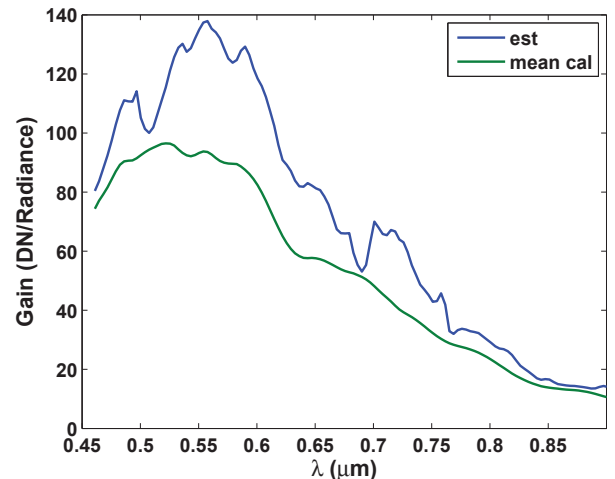

(b) Time-2

Figure 4.89: Estimated detector gain $\mathbf{a}^{(t)}$ obtained using NDVI in comparison with mean detector gain obtained from calibration data for AFRL data 


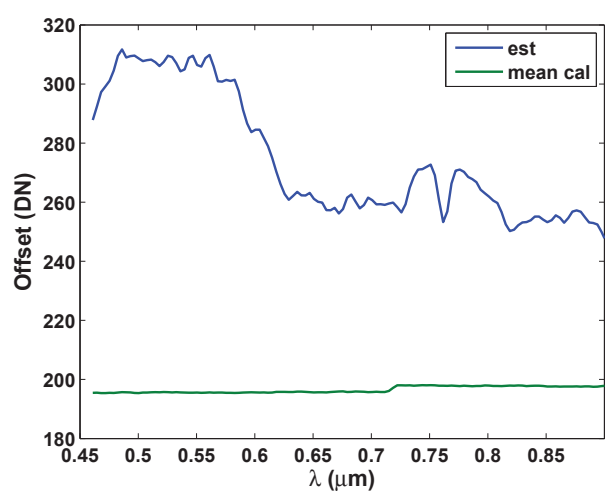

(a) Time-1

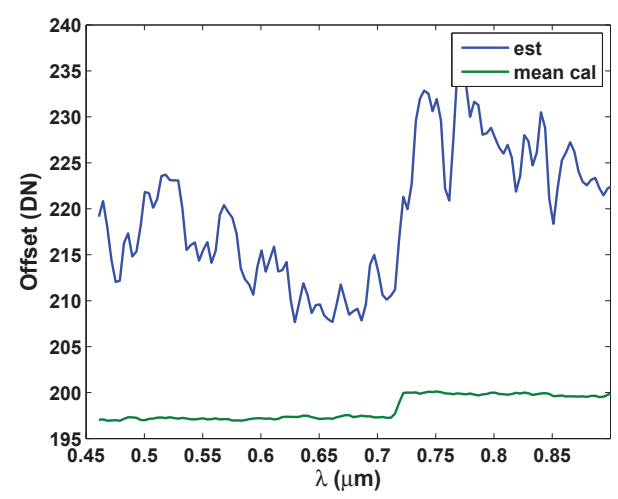

(b) Time-2

Figure 4.90: Estimated dark current offset $\mathbf{b}^{(t)}$ obtained using NDVI in comparison with mean offset obtained from calibration data for AFRL data

as that obtained from the calibration data with a larger overall magnitude. The estimated dark current offset is similarly larger than that of the calibration data. These overestimates may result from underestimated RT terms or underestimated vegetation reflectance relative to their true quantities.

Figures 4.91 and 4.92 display the final estimated detector gain and dark current offset using the NDVI initialization method. In comparison with the detector gain and dark current offset obtained from calibration data and the final estimates obtained when initializing with the calibration data as shown in Figures 4.80 - 4.83, these estimates tend to be larger in magnitude, which results from the higher initial estimates provided by the NDVI method.

Figures 4.93 and 4.94 display the final estimated shadow coefficients obtained using the NDVI initialization. The estimates are similar to those obtained for the previous case with a degree of observable discontinuity along rows near the treeline and grass region. 


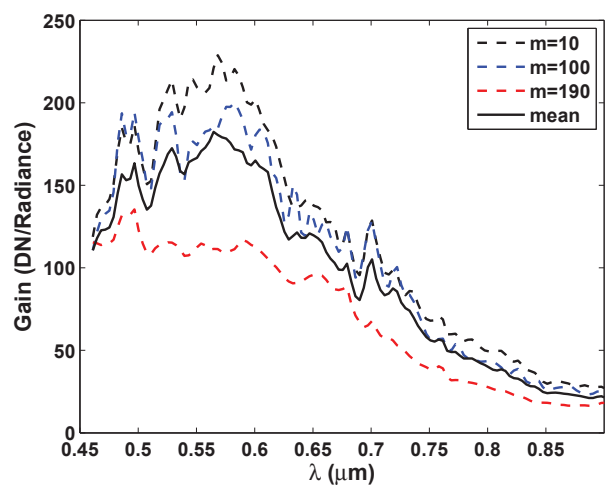

(a) Time-1

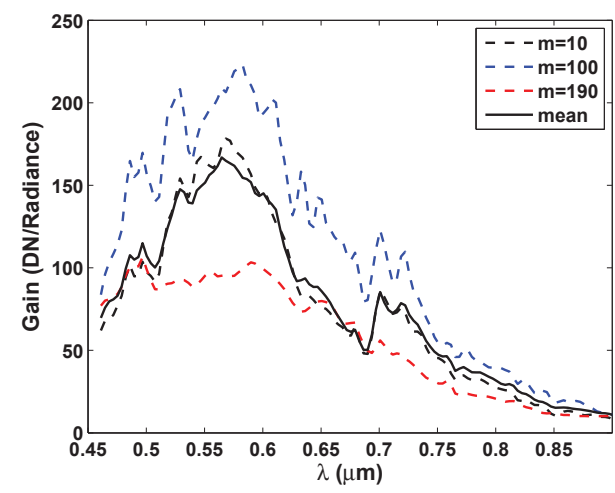

(b) Time-2

Figure 4.91: Estimated detector gain $\mathbf{a}^{(t)}$ obtained using NDVI for initialization for AFRL data

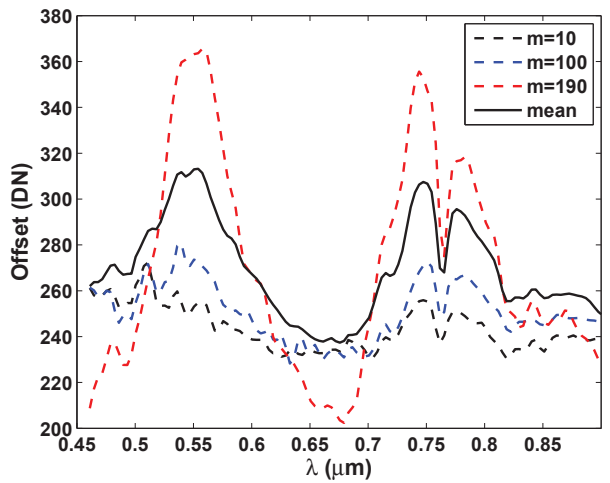

(a) Time-1

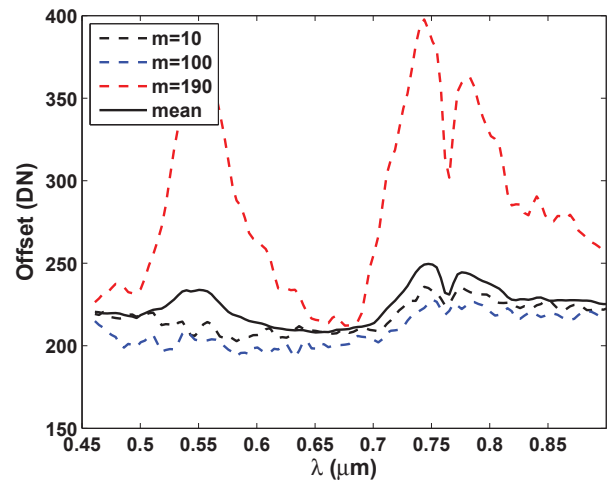

(b) Time-2

Figure 4.92: Estimated dark current offset $\mathbf{b}^{(t)}$ obtained using NDVI for initialization for AFRL data 


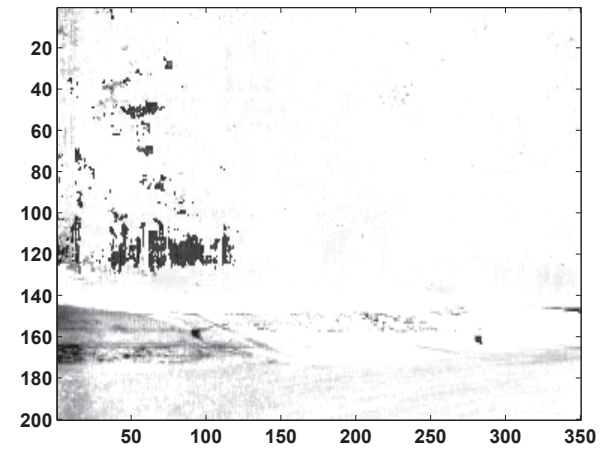

(a) $\hat{\tilde{\alpha}}^{(1)}$

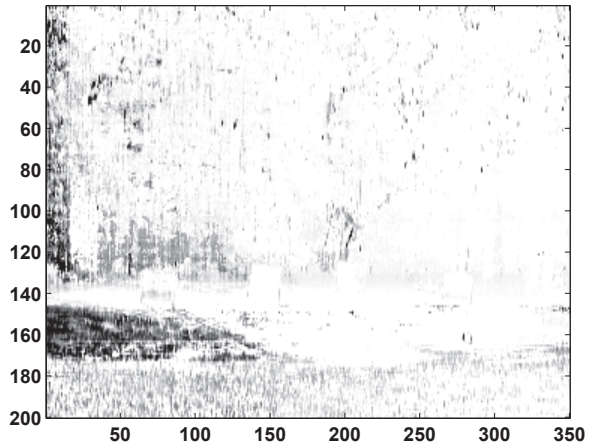

(b) $\hat{\beta}^{(1)}$

Figure 4.93: Estimated shadow coefficients for time-1 data using 5\% shadow initialization with uncalibrated data model and NDVI initialization for AFRL data

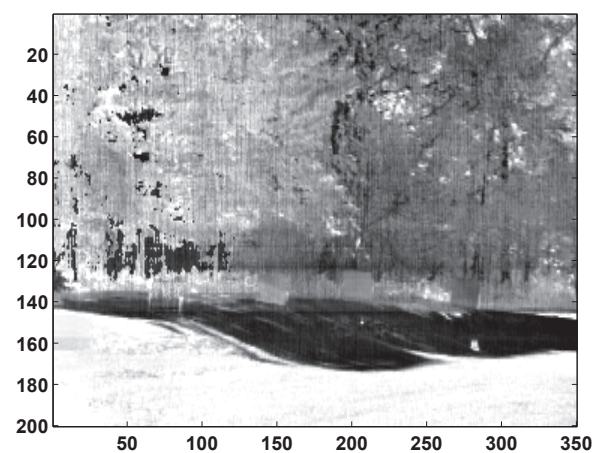

(a) $\hat{\tilde{\alpha}}^{(2)}$

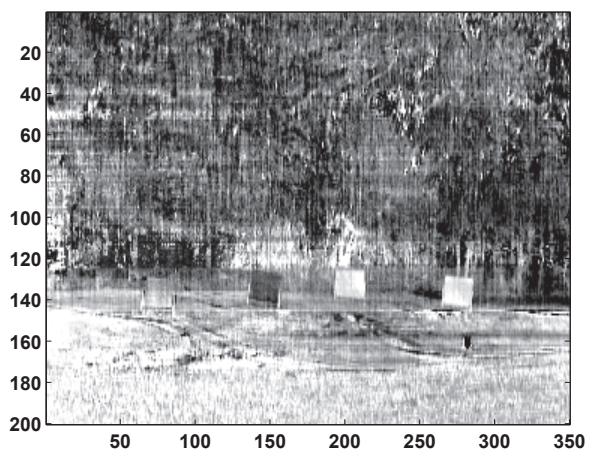

(b) $\hat{\beta}^{(2)}$

Figure 4.94: Estimated shadow coefficients for time-2 data using 5\% shadow initialization with uncalibrated data model and NDVI initialization for AFRL data 
Figure 4.95 displays the estimated reflectance for a grass pixel for the uncalibrated data model with NDVI initialization in comparison with the estimate obtained from the original calibrated data model. In this case, the estimated grass reflectance ob-

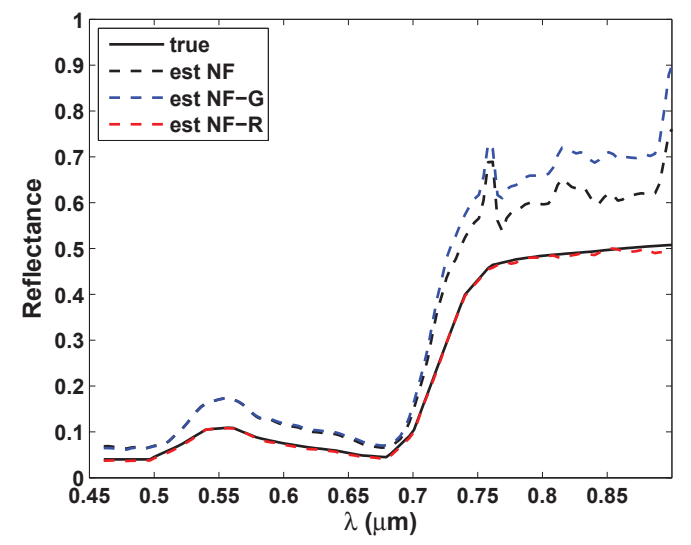

Figure 4.95: Comparison of estimated reflectance $\rho$ with true reflectance for grass pixel for baseline $\mathrm{MB}$ method on calibrated data (NF), the relatively calibrated data model (NF-G), and the uncalibrated data model with NDVI initialization (NF-R)

tained using the NDVI method is nearly identical to the lab grass reflectance provided. This results from the use of this grass reflectance spectrum as the vegetation truth for the NDVI method. Consequently, the initial detector gain and dark current offset have been "optimized" for this particular reflectance. Since the majority of the scene is vegetation, the final estimates of the detector gain and offset are similar to the initial estimates obtained from NDVI.

Figure 4.96 compares the ROC performance of the MB methods using the absolutely calibrated data model $(\mathrm{NF})$, the uncalibrated data model (NF-R), and the uncalibrated data model with NDVI initialization (NF-R-NDVI). A small decline in performance exists when using the NDVI initialization as opposed to using the initial 


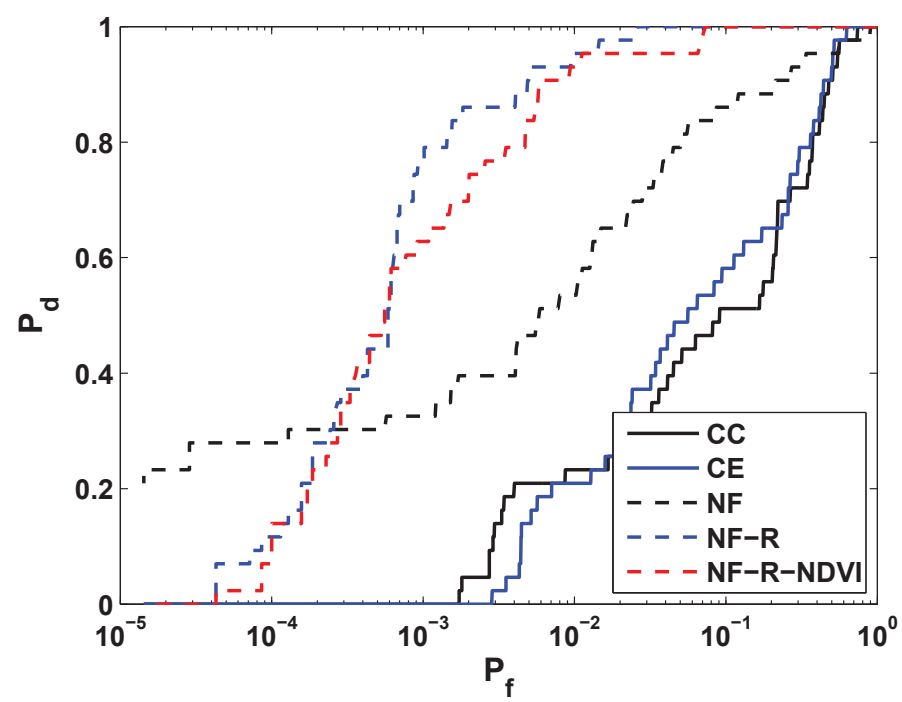

Figure 4.96: Empirical ROC curve performance of the MB methods using the absolutely calibrated data model (NF), the uncalibrated data model (NF-R), and the uncalibrated data model with NDVI initialization (NF-R-NDVI) in comparison with $\mathrm{CC}$ and $\mathrm{CE}$ algorithms for AFRL data

estimates obtained from calibration data. Figure 4.97 displays the detection statistic images of the three model-based methods. For the NDVI case, there appears to be more residual clutter in the dirt patch area described earlier. This may result from the use of vegetation for estimating the initial gain and offset, which may result in estimated detector gain and offset terms optimized for vegetation in the scene but are suboptimal for other material classes.

\subsection{HYDICE Data}

Testing on airborne hyperspectral imagery provides further real world results in a more typical downlooking remote sensing geometry. The same MB change detection 


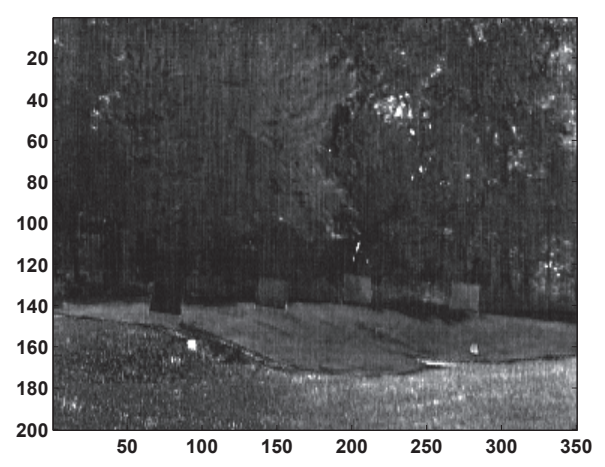

(a) Original data model (NF)

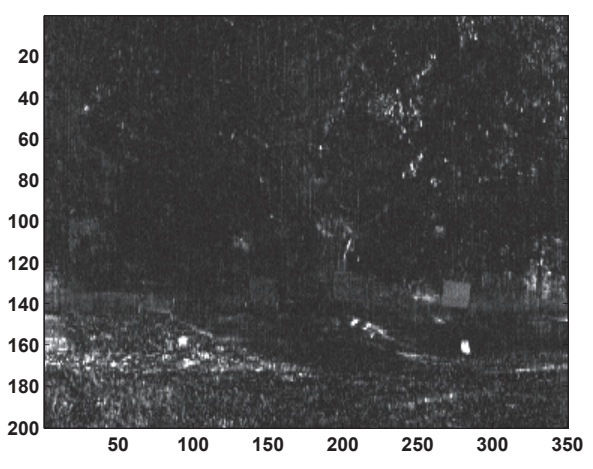

(b) Uncalibrated data model (NFR)

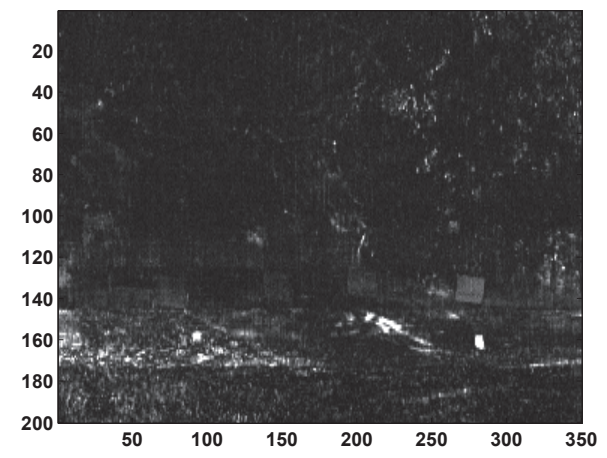

(c) Uncalibrated data model with NDVI initialization (NF-RNDVI)

Figure 4.97: Comparison of detection statistic images for the MB methods using the original data model (NF), the uncalibrated data model (NF-R), and the uncalibrated data model with NDVI initialization (NF-R-NDVI) 
experiments are applied here to HYDICE airborne imagery for further insight into the strengths and limitations associated with the MB approach.

\subsubsection{Data Summary}

The HYDICE sensor is a VNIR-SWIR pushbroom imaging spectrometer with wavelength range of $0.4-2.5 \mu \mathrm{m}$ with 210 spectral bands (nominally 10nm spectral sampling) $[4,3,68,2]$. Other useful system characteristics associated with the sensor are provided in Table 4.5. The HYDICE spectrometer uses a prism to disperse light

\begin{tabular}{|c|c|}
\hline Platform: & ERIM CV-580 \\
\hline Sensor system operating altitude: & 2000-7500m; 6000m design point \\
\hline Aircraft operating altitude: & Sea level - $7500 \mathrm{~m}$ \\
\hline Spectral Range: & $0.4-2.5$ um with 210 bands \\
\hline Aperture diameter: & $27 \mathrm{~mm}$ \\
\hline $\mathrm{f} / \#:$ & 3.0 \\
\hline Swath FOV: & $8.94^{\circ}$ \\
\hline Swath width: & 308 pixels \\
\hline IFOV: & $0.507 \mathrm{mrad}$ \\
\hline Array size: & $320 \times 210$ pixels \\
\hline Channel width (FWHM): & $7.6-16 \mathrm{~nm}$ \\
\hline InSb focal planes for three regions: & A) 0.4-1.0um B) 1.0-1.9um C) 1.9-2.5um \\
\hline Avg SNR (by region): & 217/107/40@5\% reflectance \\
\hline Pixel size: & $40 \times 40 \mu m$ \\
\hline
\end{tabular}

Table 4.5: HYDICE Sensor Information

spectrally. The dispersion angle is the degree to which the light refracts as a function of wavelength. The dispersion angle of a ray of light passing through a prism is a nonlinear function of wavelength due to the wavelength dependence of the index of refraction of the prism material. A linear dispersion would imply that the angle of refraction increases linearly as wavelength increases. Linear dispersion results in a 
uniform spectral sampling for the system. Over a relatively small wavelength range, the dispersion can be approximately linear. However, the HYDICE system has a rather large wavelength range of $0.4-2.5 \mu \mathrm{m}$. In addition to dispersion, the spectral resolution of the system is dependent on wavelength. For the AFRL in-house sensor discussed in the previous section, the spectral resolution of the diffraction grating was around $2 \mathrm{~nm}$ for the center wavelength design. Since the AFRL sensor operates in a relatively small wavelength range $(0.45-0.9 u m)$, the resolution does not vary substantially. In the HYDICE system documentation, the full-width half-max (FWHM) channel width for the system is given to be 7.6-16nm (after binning at lower wavelengths). Richard [68] provides the system spectral resolution curve as given in Figure 4.98. The dotted line in the figure most likely represents the spectral reso-

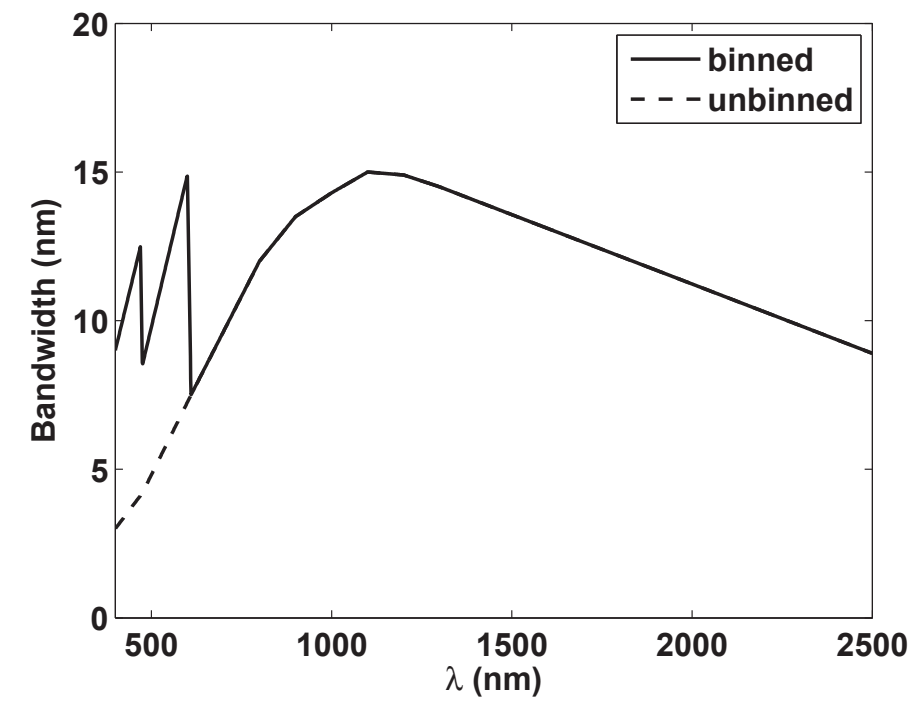

Figure 4.98: Spectral resolution of HYDICE system

lution of the system based upon the wavelength-dependent dispersion of the prism. 
Consequently, the spectral bandwidth of the unbinned data would have the smooth, continuous curve displayed in the figure. Binning is performed during post-processing on the data to improve SNR. However, specifics about binning implementation are not given in the reference materials. Around $600 \mathrm{~nm}$, the spectral bandwidth of the unbinned data is around $7.5 \mathrm{~nm}$. After binning, the bandwidth rises to $15 \mathrm{~nm}$, which suggests a bin width of 2 (i.e. averaging the two nearest spectral bands). Down near $475 \mathrm{~nm}$, the spectral bandwidth of the unbinned data is around $4.2 \mathrm{~nm}$. After binning, the spectral bandwidth rises to around $12.6 \mathrm{~nm}$, which suggests a bin width of 3. However, the type of binning filter used is not explicitly known. The MODTRAN realizations must be appropriately filtered and resampled to accurately match the HYDICE system characteristics.

The HYDICE data collection used for this study occurred at the U.S. Army's Aberdeen Proving Grounds in Maryland, which has an approximate location of 39.33 $\mathrm{N}$ latitude and $76.30^{\circ} \mathrm{W}$ longitude. Elevation in the area is around $8 \mathrm{~m}$ above sea level. The data collections occurred in 1995 on 24, 25, 26, and 29 of August. The time-1 data set was collected on 24 August near 09:10 local time. The time-2 data set was collected the following day later in the afternoon near 15:15 local time. As a result, significant illumination differences exist between the scenes. Figure 4.99 shows the selected areas for change detection testing taken from the two available scenes. The first challenge associated with the change detection problem is registering the data sets. From inspection of the images in Figure 4.99, subtle differences between the two in terms of misregistration are noticeable, including a slight vertical offset of the two scenes and a small degree of warping of the roads in the time-2 scene. In order to correct this misregistration, software developed by Space Computer Corp. 


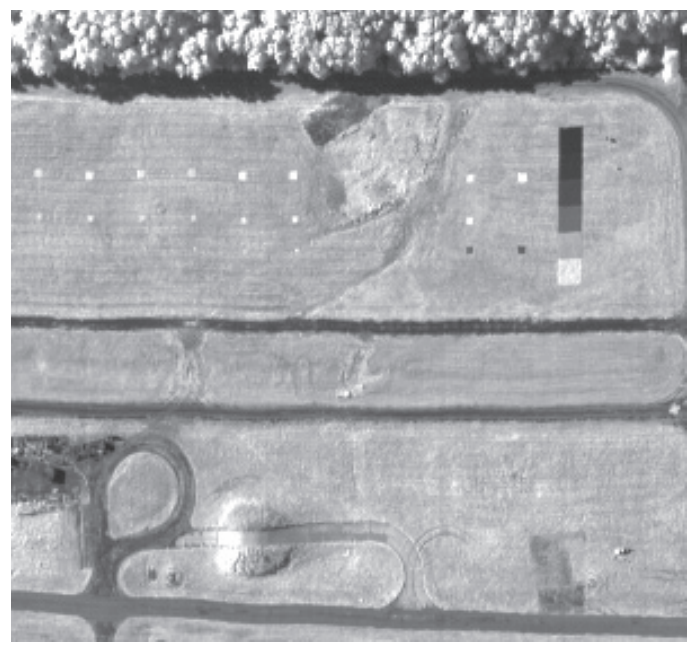

(a) Time-1: 24 August at 09:10:31 EDT

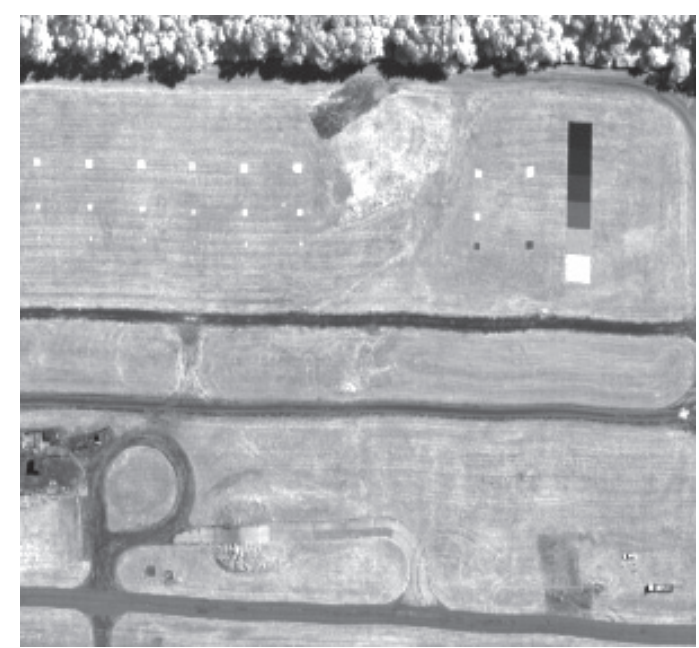

(b) Time-2: 25 August at 15:15:40 EDT

Figure 4.99: HYDICE unregistered change pair

(SCC) called In-Scene Image Stabilization (ISIS) is used [88]. The program uses tie points and/or phase correlation to bring images into subpixel level registration. The user can manually identify tie points in the imagery. Additionally, the user must specify the phase correlation box size, which is used to perform local transformations of the imagery. The interpolation used for the transformation must be specified as well. More information on the process can be found in the ISIS User's Manual [88]. Using ISIS, the images are registered and color images are shown in Figure 4.100. Change target ground truth does not exist for this data set. Consequently, the results presented here are limited to changes that can be visually identified, which precludes any sub-pixel change targets that may exist in the scene. The visually-identified change targets for this data set have been circled in the color images provided in Figure 4.100. 


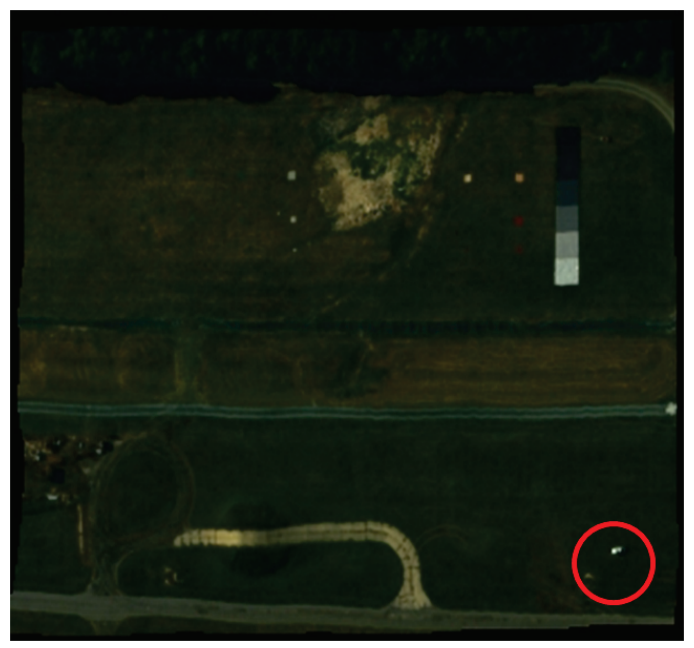

(a) Time-1

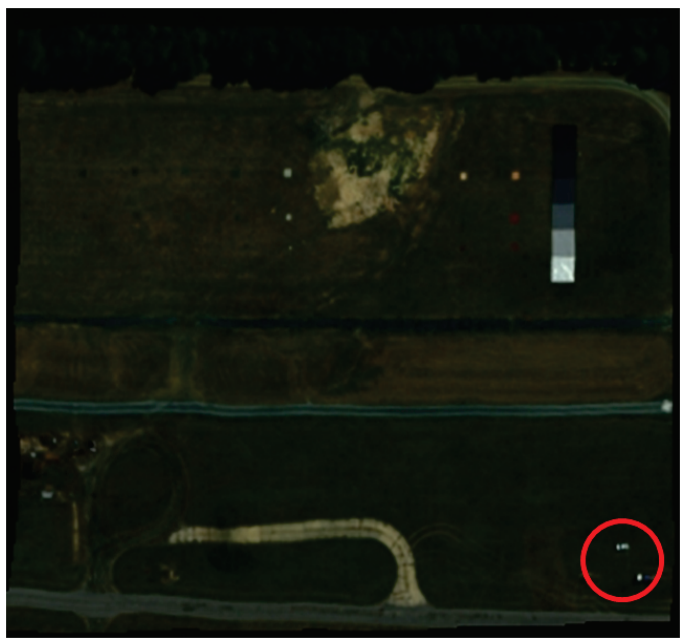

(b) Time-2

Figure 4.100: HYDICE registered change pair

In order to reduce the dimensionality of the data, strong spectral absorption regions and bands beyond $1.8 \mu \mathrm{m}$ are removed for the resized and registered data. Additionally, several bands demonstrating abnormal calibration artifacts have been removed resulting in a final data range of $0.45-1.73 \mu \mathrm{m}$ with $K=106$ spectral bands.

\subsubsection{Noise Estimation}

System noise characteristics are not available for this data set. Several of the noise estimation methods discussed in Chapter 3 are applied and compared to determine the noise gain and offset required for the noise model. First, the mean and variance of each gray calibration panel in the scene are computed to assess the relative spatial uniformity of the panels. With no ground truth available, the pixels in the scene belonging to each panel must be manually identified. Figure 4.101(a) displays the sample mean and variance of each panel for a particular band in the visual wavelength region. The variance associated with the $64 \%$ gray reflector is abnormally 
high suggesting the existence of substantial spatial non-uniformity and hence exclusion from noise analysis. Removing this panel, the sample mean and variance are plotted and compared with those obtained using Green's pixel differencing approach. Figure 4.101(b) displays the results. Typically, Green's method produces lower vari-

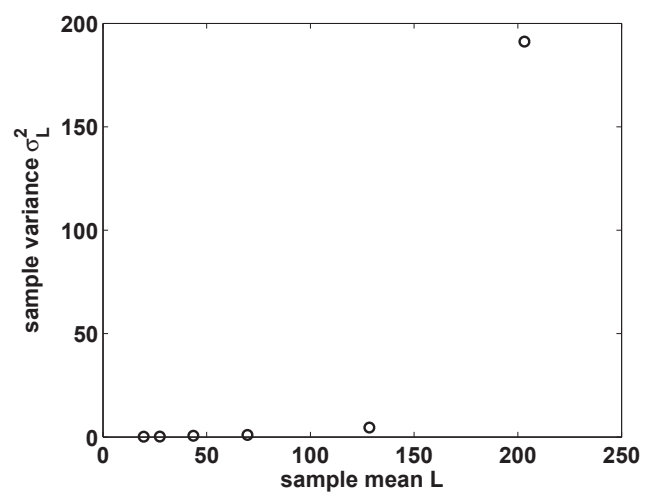

(a) Sample mean and variance computed for all panels

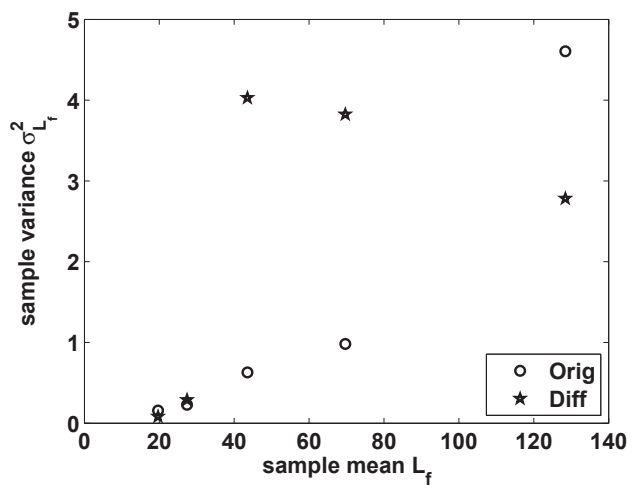

\section{(b) Compare with Green's method for first 5 panels}

Figure 4.101: Sample mean and variance of gray-level panels for a particular band in visual regime of HYDICE data

ance estimates than the non-differencing approach. However, this result does not occur for all the panels in the scene. This suggests that the pixel assignment for each panel may be incorrect or there simply are not enough pixels on each panel to produce viable statistics.

The noise estimates produced by the automated scatter plot method discussed in the previous chapter are compared with manual variance estimates obtained from the difference data (Green's method) and the original data. For the automated scatter plot method, the parameters $W=5$, and $N_{b}=20$ are used. Figure 4.102 displays the estimated gain and offset for each band using this procedure. In this case, many of the 


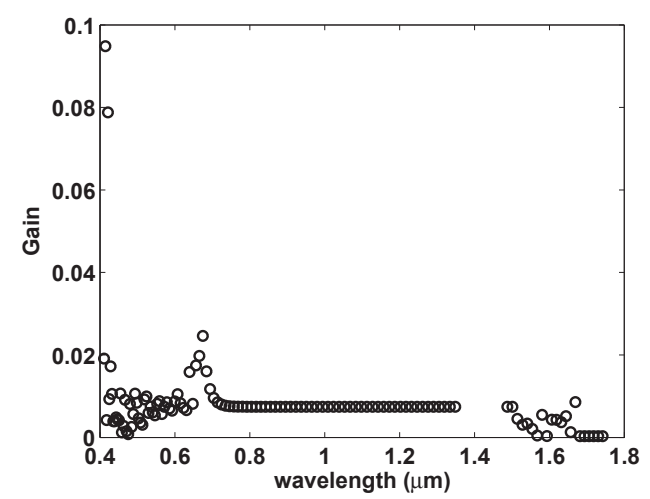

(a) Gain $\hat{a}_{L_{f}}$

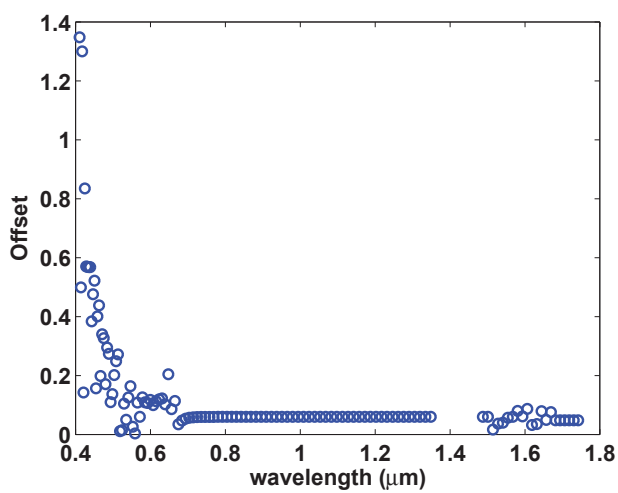

(b) Offset $\hat{b}_{L_{f}}$

Figure 4.102: Estimated gain and offset for noise model using automated scatter plot fitting method for HYDICE data

estimated gain and offset terms were non-positive, meaning that a good fit to the data did not exist. Consequently, these estimates are replaced by interpolated estimates obtained from neighboring positive estimates. Figure 4.103 displays the noise model gain and offset estimated using the sample variance obtained from the original data (excluding 64\% gray panel). Figure 4.104 displays the estimated gain and offset obtained when the sample variance is estimated from neighbor difference pixels as done in Green's method. No consistency exists between any of the noise estimates. Additionally, many of the gain and offset terms associated with the manual variance estimates are negative. As a result, no confidence in the noise estimates obtained for this data set exists and a white noise model is applied for this data set.

\subsubsection{Results using Absolutely Calibrated Data Model}

The MB algorithm is applied to this data set for a number of different configurations and initializations. For these simulations, a RT subspace dimensionality of 


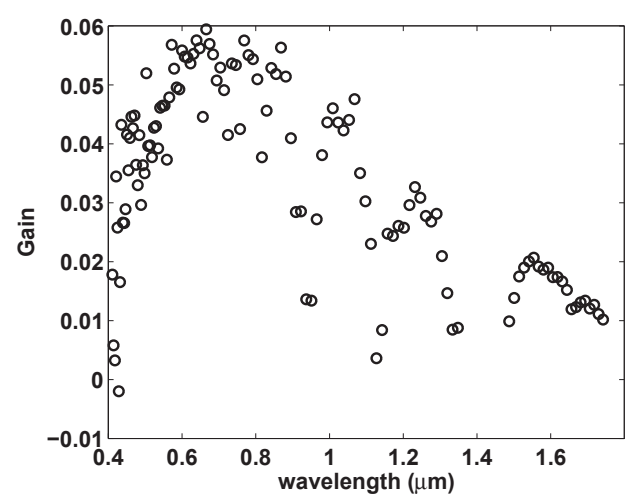

(a) Gain $\hat{a}_{L_{f}}$

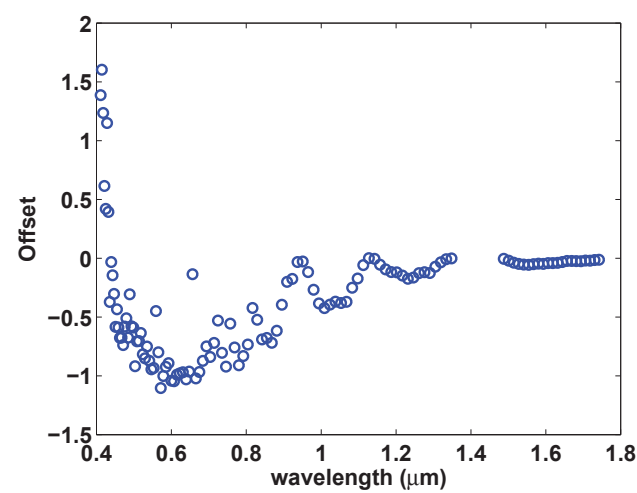

(b) Offset $\hat{b}_{L_{f}}$

Figure 4.103: Estimated gain and offset for noise model using fit to sample mean and sample variance of gray panels for HYDICE data

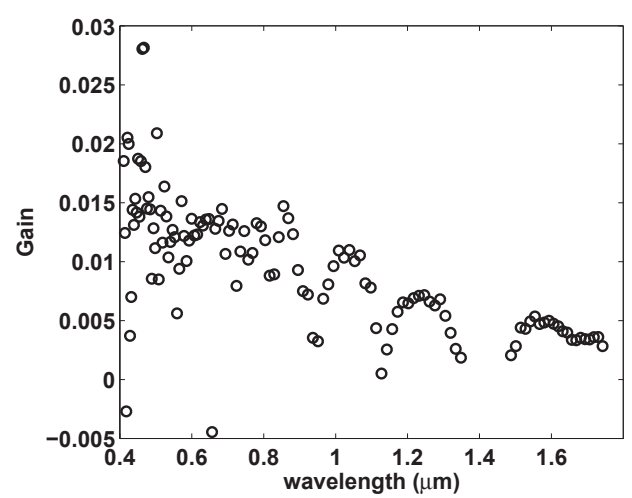

(a) Gain $\hat{a}_{L_{f}}$

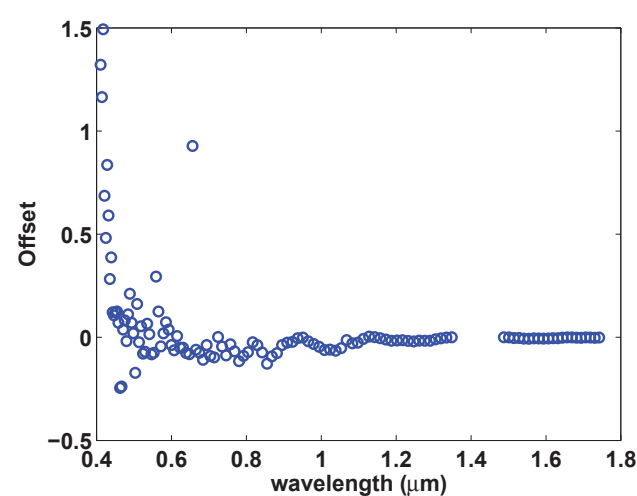

(b) Offset $\hat{b}_{L_{f}}$

Figure 4.104: Estimated gain and offset for noise model using fit to sample mean and sample variance of gray panels. Sample variance estimated using Green's method for HYDICE data

$I=10$ is used. The RT basis coefficients are initialized using MODTRAN simulations with input parameters that are expected to be representative of the data collection conditions. A mid-latitude summer atmospheric model is used along with a rural 
haze model with $20 \mathrm{~km}$ visibility using the default water vapor column for both data sets. The sensor altitude for both simulations is $1.524 \mathrm{~km}$. The cosine projection term is initialized using the known solar position at the time of collection. From the known GPS scene coordinates and time of day, the solar zenith angles are $\theta_{s}^{(1)}=59.4^{\circ}$ and $\theta_{s}^{(2)}=40.3^{\circ}$. Reflectance truth for several objects in the scene exists, which can be used to examine initialization and estimation performance. However, the accuracy of the reflectance truth is suspect due to high variability among the samples provided and the presence of atmospheric artifacts in the spectra, which is demonstrated in the grass spectrum and $64 \%$ gray panel spectrum provided in Figure 4.105(a). These library reflectance spectra are assumed to be close enough to truth to provide useful information in regard to performance. The initial estimates of the expected radiance for the $64 \%$ gray calibration panel using the initial RT estimates and using the gray panel reflectance spectrum are displayed in Figure 4.105(b) along with the actual measured radiance for a panel pixel. The initial estimates are relatively accurate for

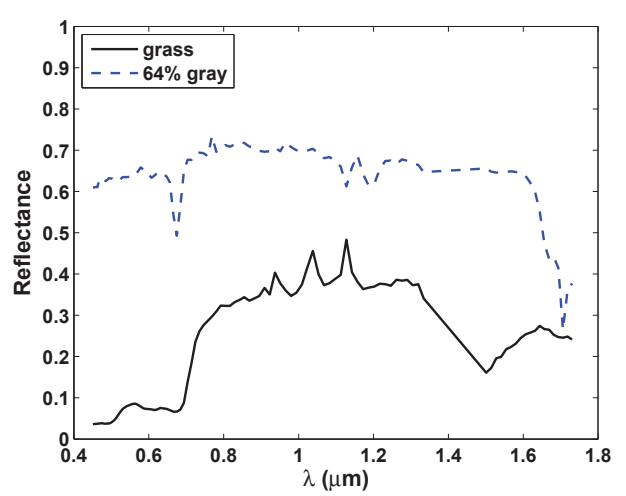

(a) Reflectance truth

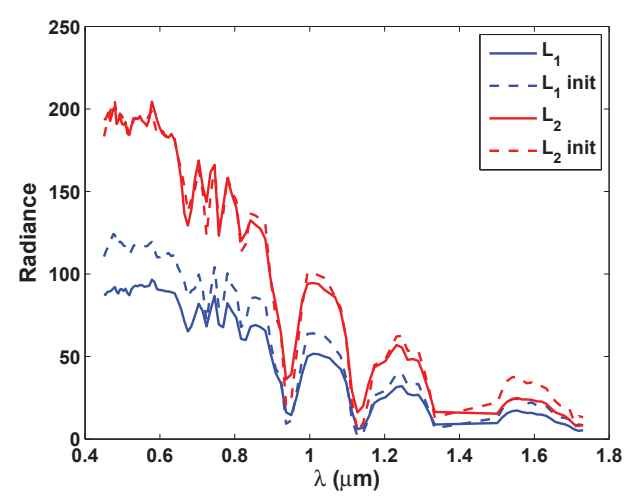

(b) Initial estimates for $64 \%$ gray panel

Figure 4.105: Material reflectance truth and initial radiance estimates for HYDICE data 
the time-2 data but over-estimated for the time-1 data.

The estimation and detection results are compared for the MB algorithm run in the three different reflectance estimation modalities as in the previous case. The algorithm is run using 20 iterations. Figure 4.106 displays the convergence criteria given in (2.56) and (2.57) for one particular case for the HYDICE data. Both of the

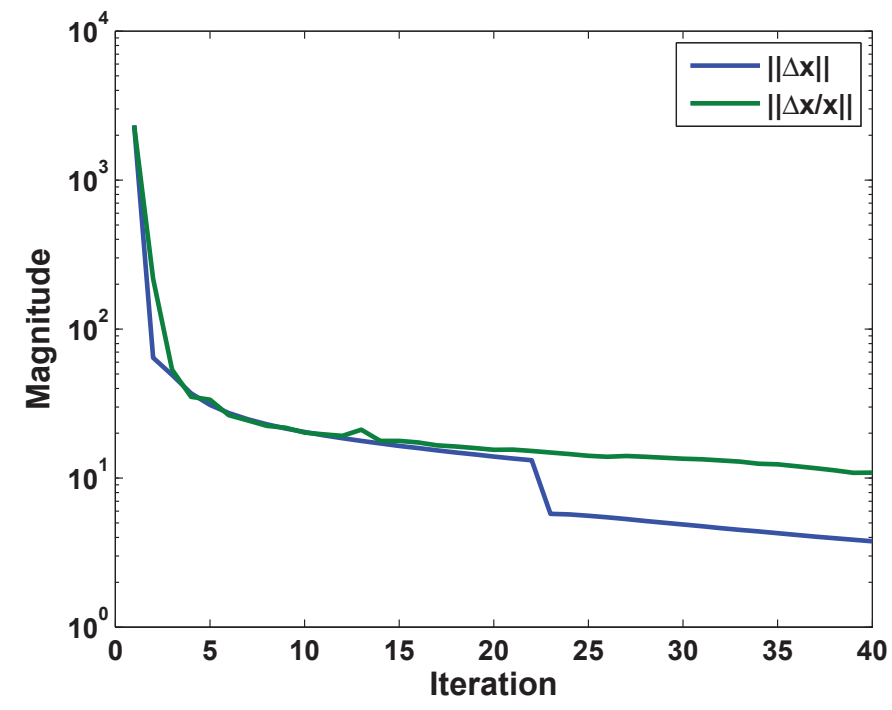

Figure 4.106: Convergence criteria as a function of iteration for HYDICE data

convergence criteria decrease in general. Choosing a threshold to stop iterating is difficult. No appreciable change in detection performance is observed for iterations beyond 20. As a result, 20 iterations are applied for testing of HYDICE data.

For the reflectance smoothing implementations of the algorithm, a Bartlett window of length 5 is used as the data is relatively smooth to begin with due to lower spectral resolution. As before, a reflectance subspace dimension of $I_{r}=40$ is used when implementing the Legendre subspace representation. 


\section{$5 \%$ Shadow Initialization}

The histogram threshold method is used to detect the bottom $5 \%$ of spectral norm pixels in both the time- 1 and time- 2 scenes. These pixels are detected as shadow pixels and initialized with the shadow coefficient values of $\alpha=0.1$ and $\beta=0.5$. Figures 4.107 and 4.108 display the initial shadow coefficient estimates for the time-1 and time-2 data respectively. Shadow truth does not exist for this data. Consequently,

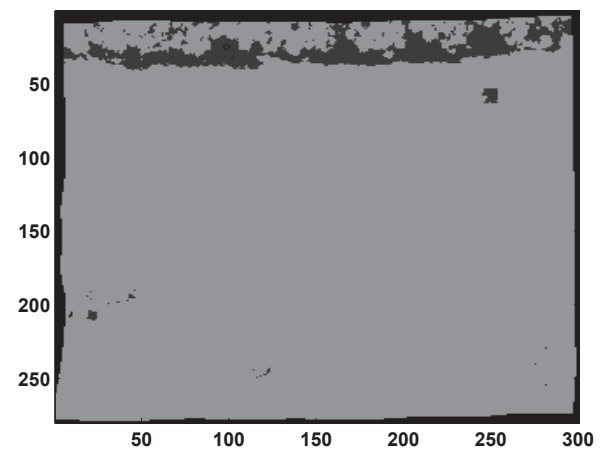

(a) $\tilde{\alpha}^{(1)}$

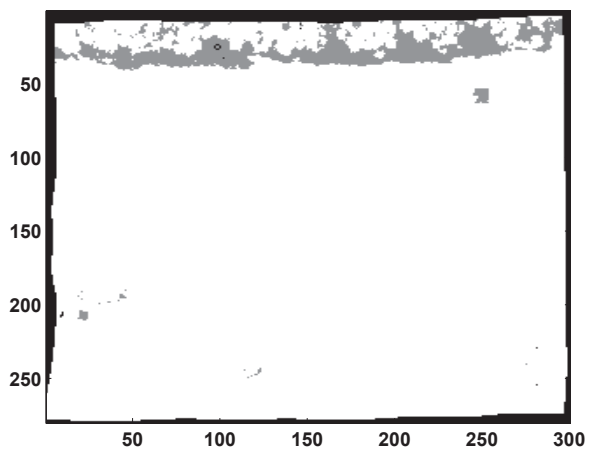

(b) $\beta^{(1)}$

Figure 4.107: Initial estimates for time-1 shadow coefficients using bottom $5 \%$ of spectral norm pixels for HYDICE data

shadow initialization/estimates are compared with shadow regions visually identified in the scene color images shown in Figure 4.100. The treeline/canopy shadows are identified using this initialization along with a couple of the darker gray calibration panels.

Detection and estimation results are obtained using these initial shadow and RT estimates. Unfortunately, the true RT terms and shadow coefficients are unknown. As a result, the estimation performance of the $\mathrm{MB}$ algorithm for these terms cannot 


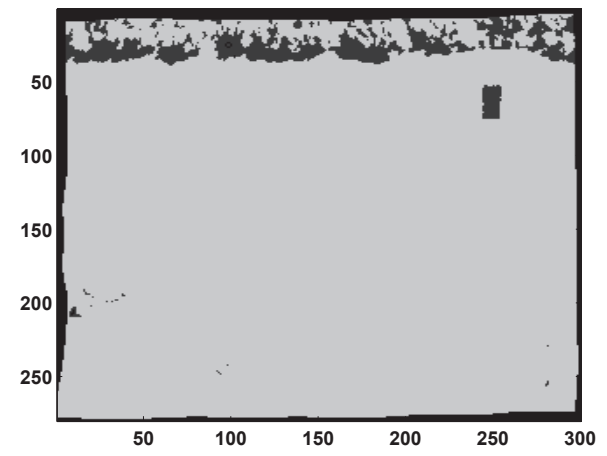

(a) $\tilde{\alpha}^{(2)}$

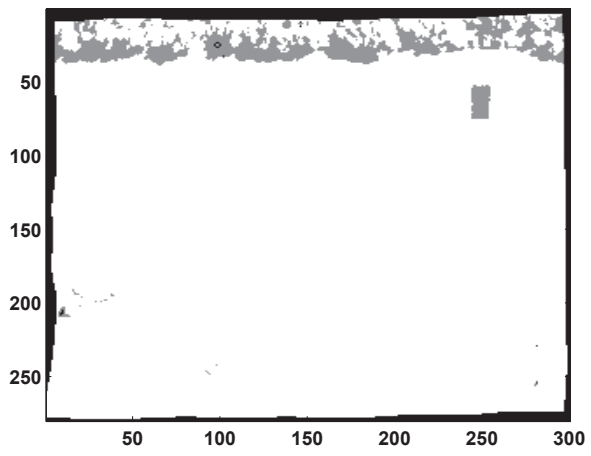

(b) $\beta^{(2)}$

Figure 4.108: Initial estimates for time-2 shadow coefficients using bottom $5 \%$ of spectral norm pixels for HYDICE data

be quantified. However, the estimated RT terms can be compared with various MODTRAN realizations used to create the RT subspace as a sanity check on the estimates. For this initialization, the estimated time-1 RT parameters are nearest to several realizations generated using a mid-latitude summer atmosphere with $25 \mathrm{~km}$ visibility and 1.4 times the default water vapor column associated with this atmospheric model. Figure 4.109 displays the RT estimates for time-1 for the three different reflectance estimation modalities. The estimated time-2 RT terms are closest to MODTRAN realizations generated using a tropical atmospheric model with $30 \mathrm{~km}$ visibility and the default water vapor column. Figure 4.110 displays the RT estimates for time-2 for the three different reflectance estimation modalities compared with the nearest MODTRAN realization.

The shadow coefficient estimates using the various reflectance estimation modalities are all very similar. Consequently, only the results for the baseline non-filtering method are shown. Figures 4.111 and 4.112 display the shadow estimates for the 


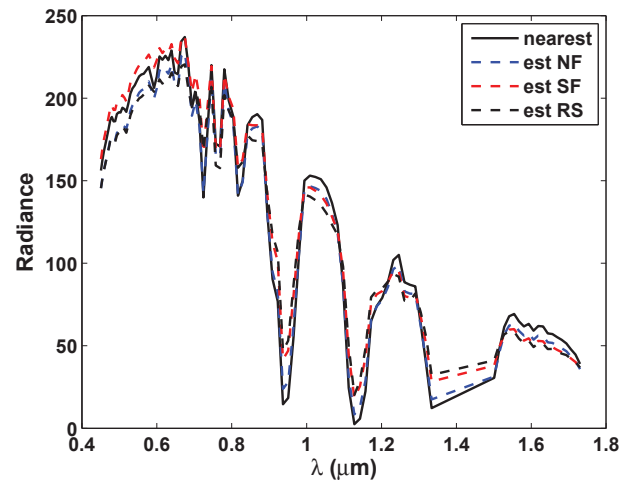

(a) $\boldsymbol{\tau} \odot \mathbf{L}_{s}$

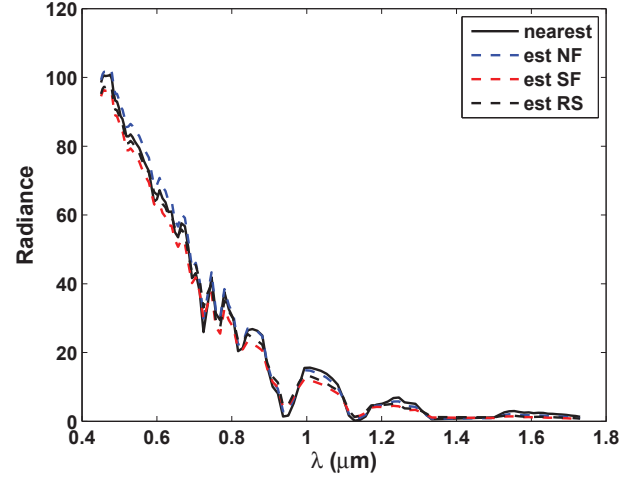

(b) $\boldsymbol{\tau} \odot \mathbf{L}_{d}$

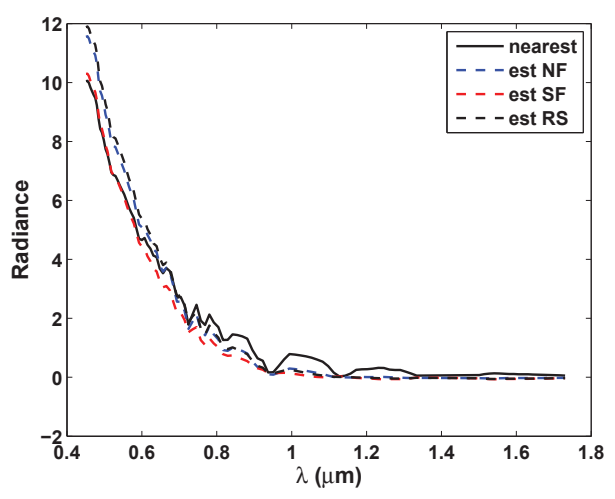

(c) $\mathbf{L}_{p}$

Figure 4.109: RT estimates for time-1 HYDICE data for different reflectance estimation modalities using $5 \%$ shadow initialization 


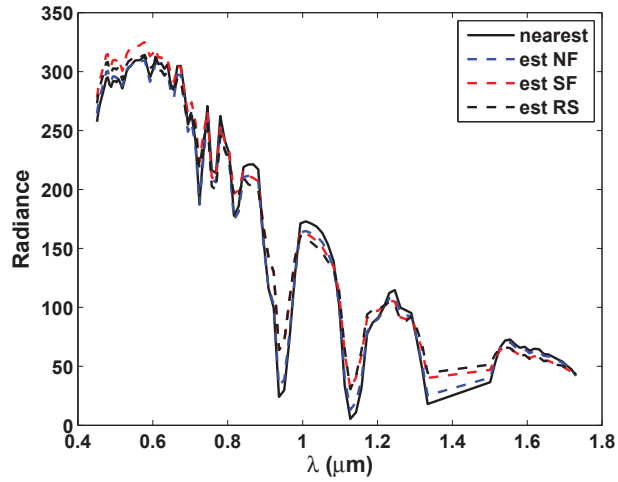

(a) $\boldsymbol{\tau} \odot \mathbf{L}_{s}$

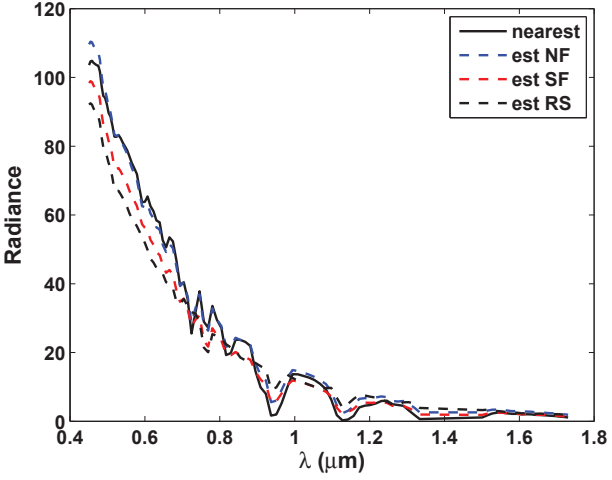

(b) $\boldsymbol{\tau} \odot \mathbf{L}_{d}$

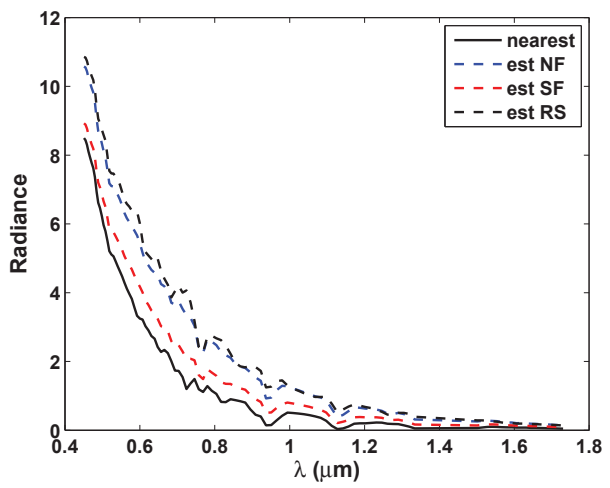

(c) $\mathbf{L}_{p}$

Figure 4.110: RT estimates for time-2 HYDICE data for different reflectance estimation modalities using $5 \%$ shadow initialization. 
time- 1 and time- 2 data. Both the time- 1 and time- 2 estimates correlate well with

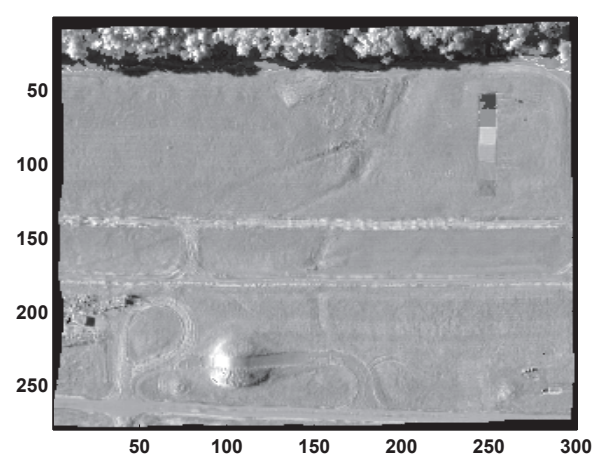

(a) $\hat{\tilde{\alpha}}^{(1)}$

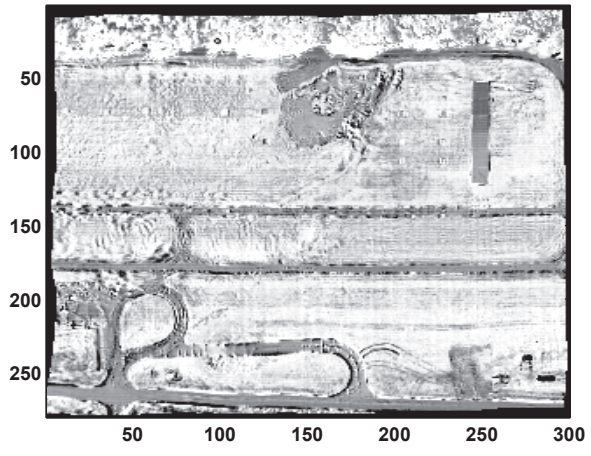

(b) $\hat{\beta}^{(1)}$

Figure 4.111: Estimated shadow coefficients for time-1 data using 5\% shadow initialization for HYDICE data

the shadows observed in the color imagery.

The residual estimation error resulting from model parameter estimates is examined. Figure 4.113 displays the estimated measurement $\hat{L}$ for a grass pixel for both the time- 1 and time-2 data. Figure 4.114 displays the same for a pixel located on the $64 \%$ gray panel. The residual optimization error exists primarily in the atmospheric absorption bands for the data. Figure 4.115 displays the reflectance estimates associated with these pixels in comparison with laboratory truth. In general, the reflectance estimates have the same general shape as the true reflectance. The non-filtered reflectance estimates demonstrate much stronger residual atmospheric absorption phenomena than those obtained using the spectral filtering method and the subspace constrained method. Since the non-filtered method does not constrain the smoothness of the reflectance estimate, the estimate possesses absorption features 


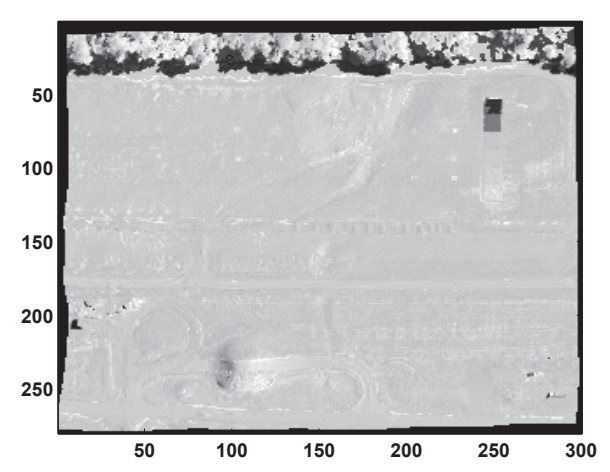

(a) $\hat{\tilde{\alpha}}^{(2)}$

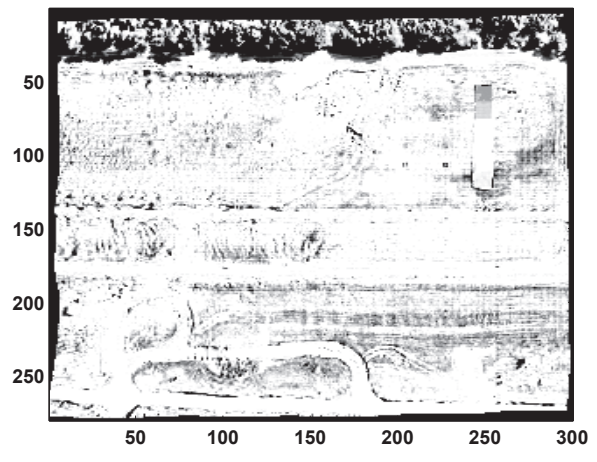

(b) $\hat{\beta}^{(2)}$

Figure 4.112: Estimated shadow coefficients for time-2 data using 5\% shadow initialization for HYDICE data

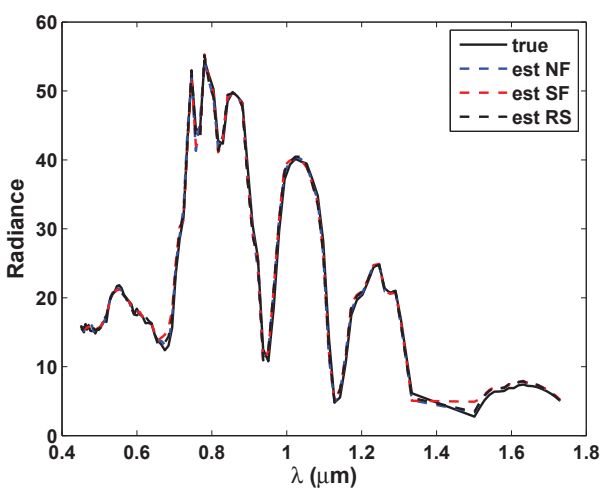

(a) Time-1

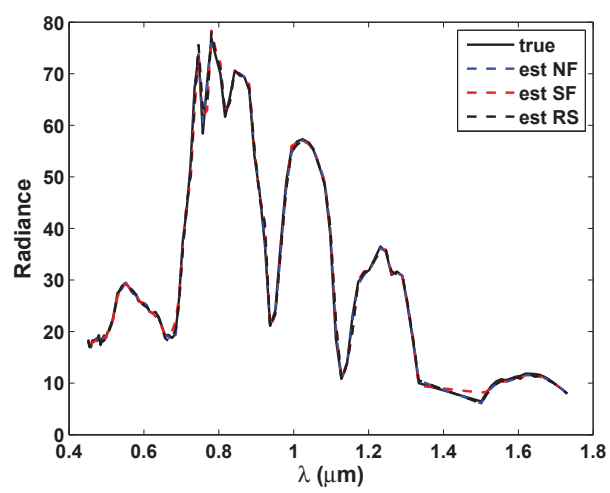

(b) Time-2

Figure 4.113: Comparison of estimated measurement $\hat{L}$ with true measurement $L$ for grass pixel using $5 \%$ shadow initialization for HYDICE data 


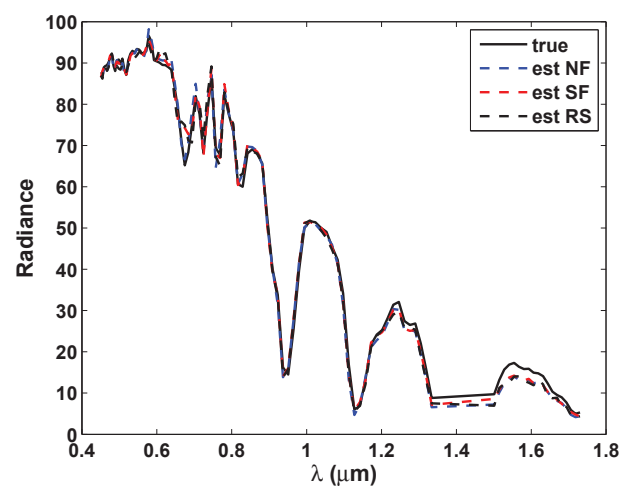

(a) Time-1

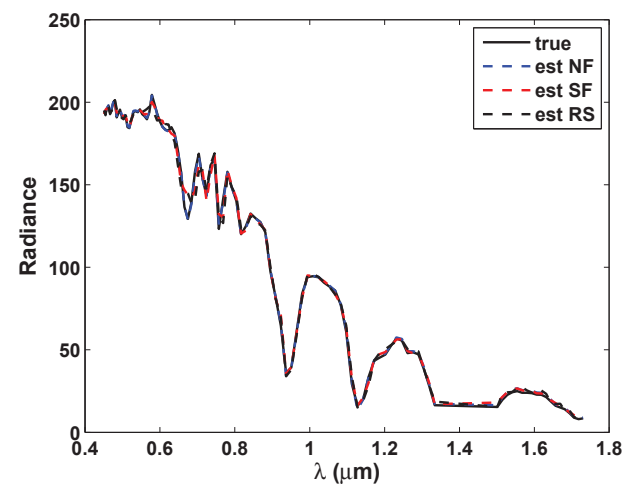

(b) Time-2

Figure 4.114: Comparison of estimated measurement $L$ with true measurement for $64 \%$ gray panel pixel using 5\% shadow initialization for HYDICE data

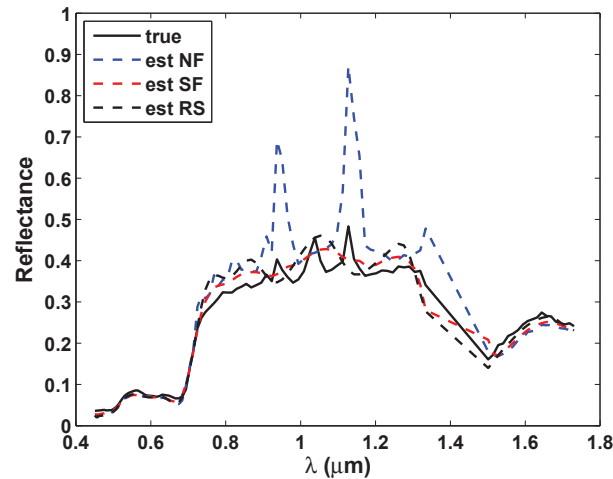

(a) Grass

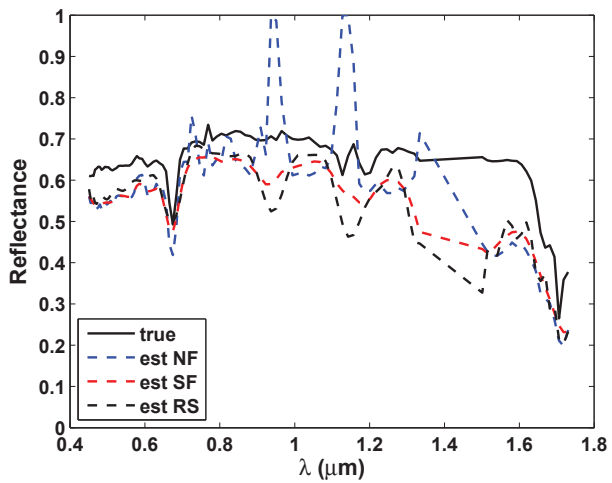

(b) $64 \%$ gray panel

Figure 4.115: Comparison of estimated reflectance $\rho$ with true reflectance for grass pixel and $64 \%$ gray panel using $5 \%$ shadow initialization for HYDICE data 
as these are used to reduce the residual optimization error resulting in these regions. The reflectance estimates for the grass pixel obtained using the spectral filtering and subspace constrained methods are close to the true spectrum. However, the estimates for the gray panel have a higher degree of error, especially in the SWIR spectral region. The error may result from estimation error associated with the RT terms, which are amplified by the higher reflectance of the gray panel.

Figure 4.116 displays the empirical ROC curve performance of the MB algorithm using the three reflectance estimation modalities in comparison with the performance of the $\mathrm{CC}$ and $\mathrm{CE}$ change detection algorithms discussed. In general, the MB methods

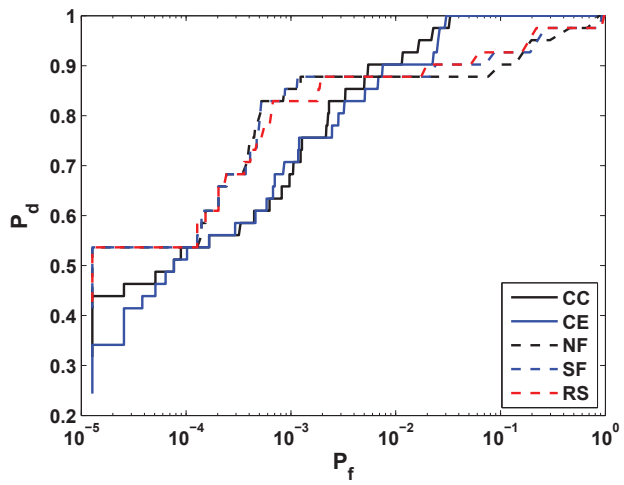

Figure 4.116: Empirical ROC curve performance for different reflectance estimation modalities using $5 \%$ shadow initialization in comparison with $\mathrm{CC}$ and $\mathrm{CE}$ algorithms for HYDICE data

achieve higher performance at lower false alarm rates than the $\mathrm{CC}$ and $\mathrm{CE}$ algorithms. Minimal difference in ROC performance exists between the different modalities of the MB method. Figure 4.117 displays the detection statistic images of the CC and CE algorithms respectively. These algorithms demonstrate clutter leakage in the shadow 


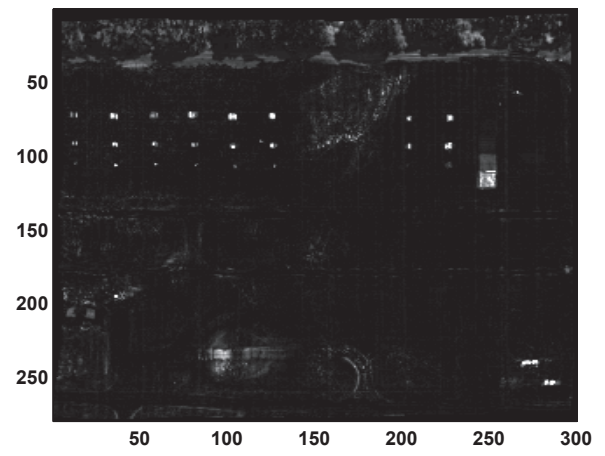

(a) $\mathrm{CC}$

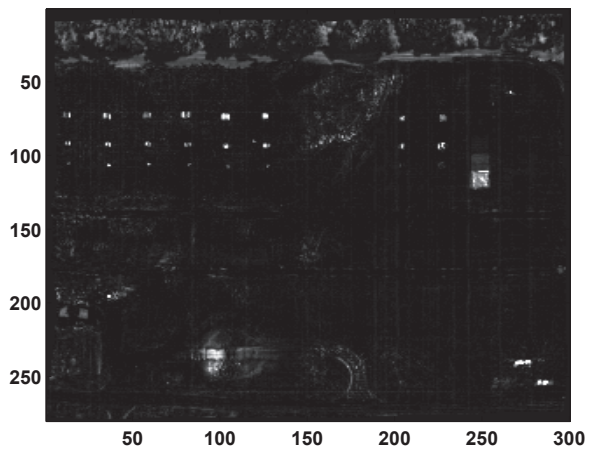

(b) $\mathrm{CE}$

Figure 4.117: Comparison of detection statistic images for $\mathrm{CC}$ and $\mathrm{CE}$ change detection algorithms for HYDICE data

regions as well as the small fabric target regions and the small mound near the bottom of the scene where a noticeable illumination change occurs. Figure 4.118 displays the detection statistic images for the three reflectance modalities of the MB method. The dynamic range of the images is stretched between 0 and $\mu+14 \sigma$. Visually, the $\mathrm{MB}$ methods improve clutter suppression, especially in the regions described above where the CC and CE algorithms struggle. Particularly, the clutter leakage associated with the small mound is completely removed when using the MB methods. Residual clutter does exists for several of the fabric targets, the $64 \%$ gray panel, and a several portions near road edges at the bottom of the scene. This residual clutter can result from model mismatch, inaccurate RT estimates, or misregistration that exists between the two images. 


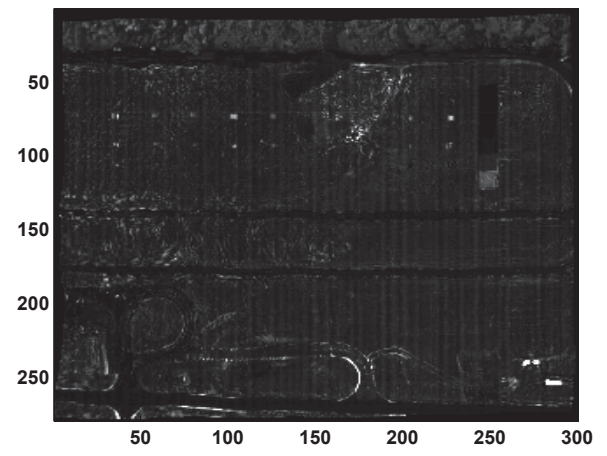

(a) No reflectance filtering

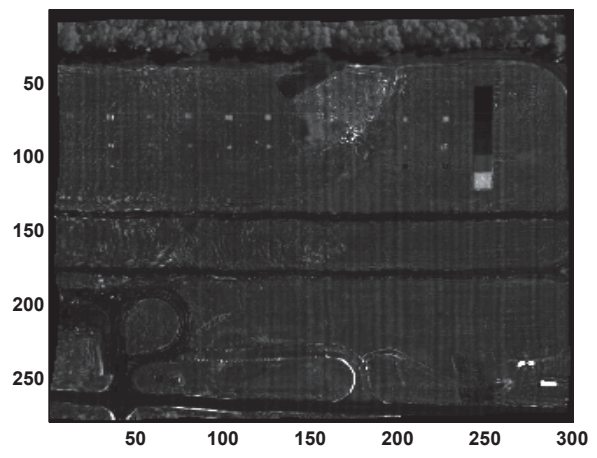

(b) Reflectance filtering

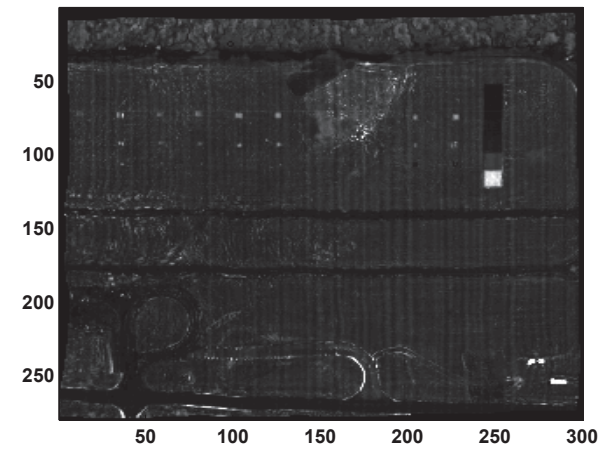

(c) Reflectance subspace

Figure 4.118: Comparison of detection statistic images for different MB reflectance modalities using $5 \%$ shadow initialization for HYDICE data 


\section{0\% Shadow Initialization}

The histogram threshold method is used to detect the bottom $10 \%$ of spectral norm pixels in both the time- 1 and time- 2 scenes. These pixels are detected as shadow pixels and initialized with the shadow coefficient values given above. Figures 4.119 and 4.120 display the initial shadow coefficient estimates for the time-1 and time-2 data respectively. The initial shadow estimates now begin to identify darker

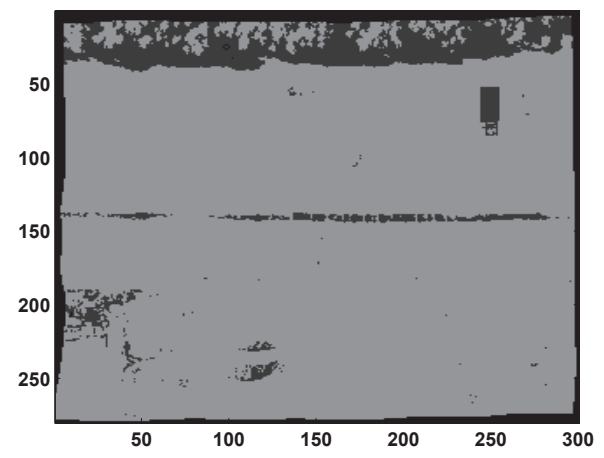

(a) $\tilde{\alpha}^{(1)}$

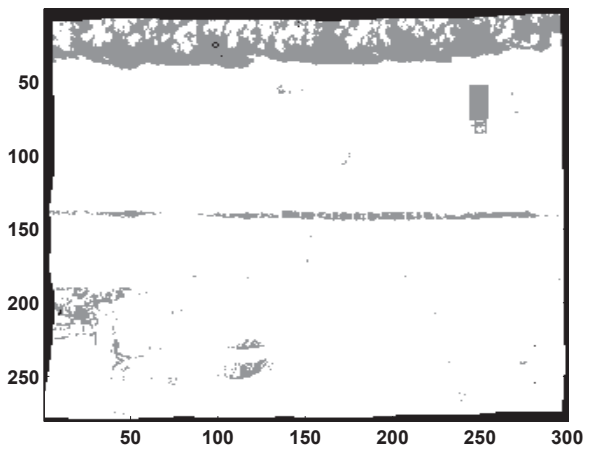

(b) $\beta^{(1)}$

Figure 4.119: Initial estimates for time-1 shadow coefficients using bottom $10 \%$ of spectral norm pixels for HYDICE data

road portions and more of the dark gray calibration panels as shadows pixels.

Detection and estimation results are obtained using these initial shadow and RT estimates. The RT estimates shown in Figures 4.121 and 4.122 are nearly the same as the previous shadow initialization, demonstrating a relative insensitivity to the shadow initialization used.

The shadow estimates using the various reflectance estimation modalities are all very similar. Consequently, only the results for the baseline non-filtering method are 


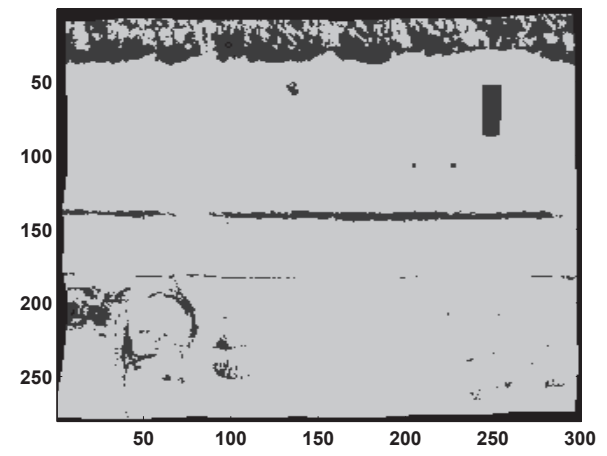

(a) $\tilde{\alpha}^{(2)}$

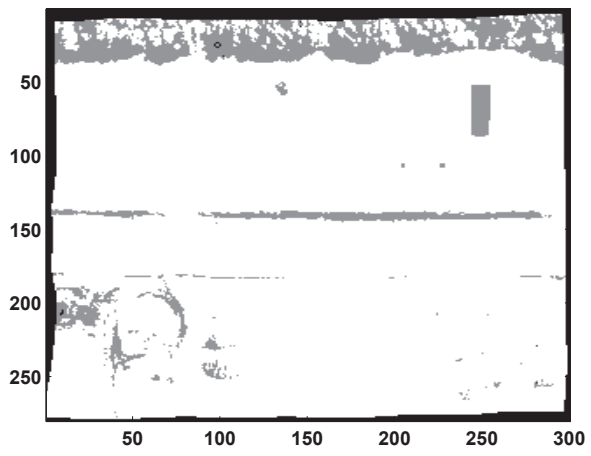

(b) $\beta^{(2)}$

Figure 4.120: Initial estimates for time-2 shadow coefficients using bottom $10 \%$ of spectral norm pixels for AFRL data

shown. Figures 4.123 and 4.124 display the shadow estimates for the time- 1 and time2 data. The shadow estimates obtained in this case are similar to those obtained for the $5 \%$ case with the exception that those additional pixels detected initially as shadows remain as shadow pixels.

The residual estimation error results and reflectance estimates shown in Figures $4.125,4.126$ and 4.127 are all very similar to the results obtained with the $5 \%$ shadow initialization.

Figure 4.128 displays the empirical ROC curve performance of the MB algorithm using the three reflectance estimation modalities in comparison with the performance of the $\mathrm{CC}$ and $\mathrm{CE}$ change detection algorithms discussed. In general, performance is the same as with the previous shadow initialization. The detection statistic images shown in Figure 4.129 are similar as well. 


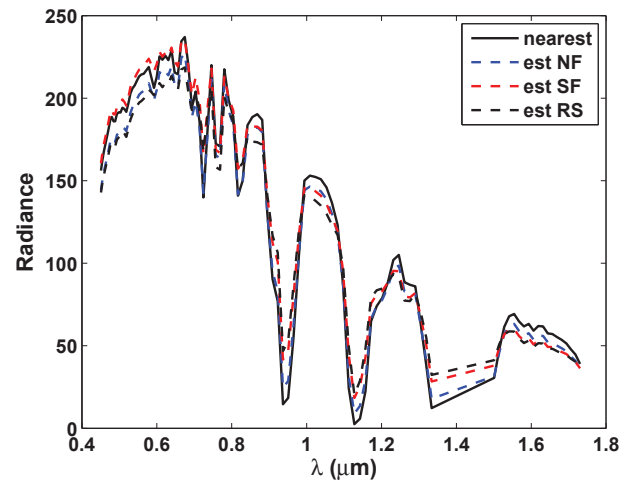

(a) $\boldsymbol{\tau} \odot \mathbf{L}_{s}$

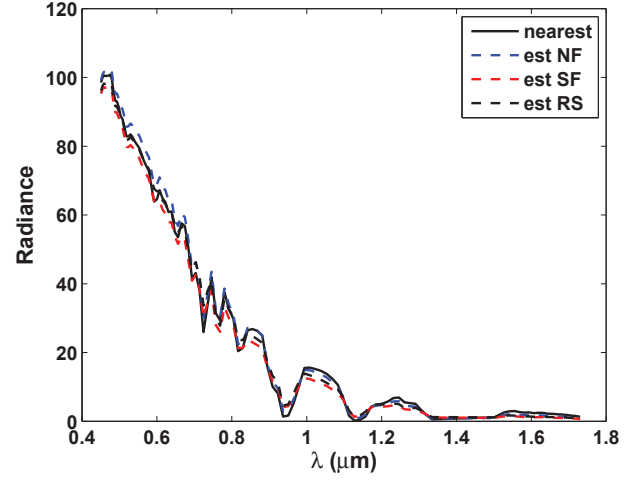

(b) $\boldsymbol{\tau} \odot \mathbf{L}_{d}$

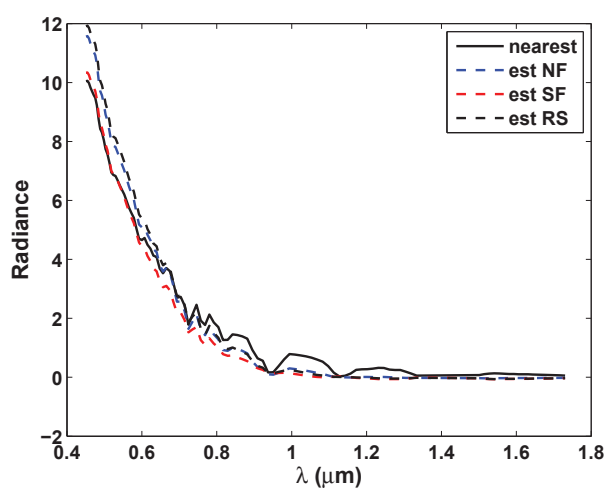

(c) $\mathbf{L}_{p}$

Figure 4.121: RT estimates for time-1 HYDICE data for different reflectance estimation modalities using $10 \%$ shadow initialization. 


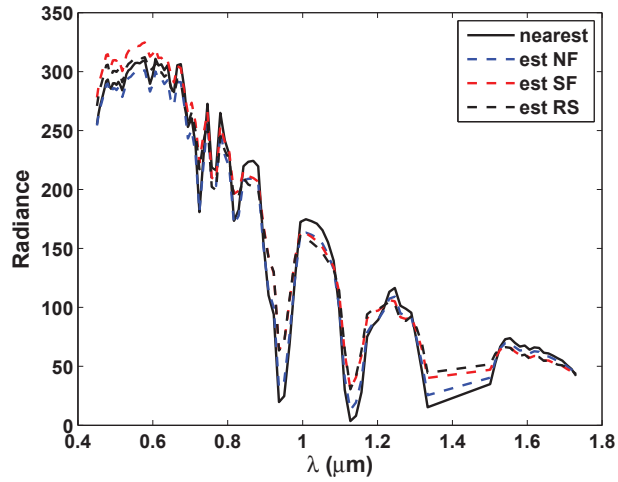

(a) $\boldsymbol{\tau} \odot \mathbf{L}_{s}$

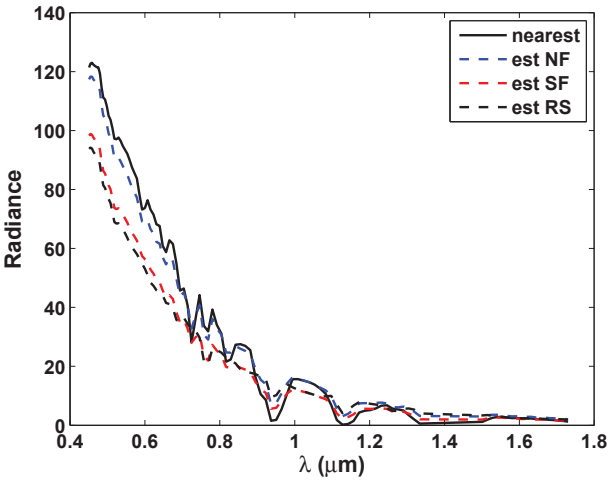

(b) $\boldsymbol{\tau} \odot \mathbf{L}_{d}$

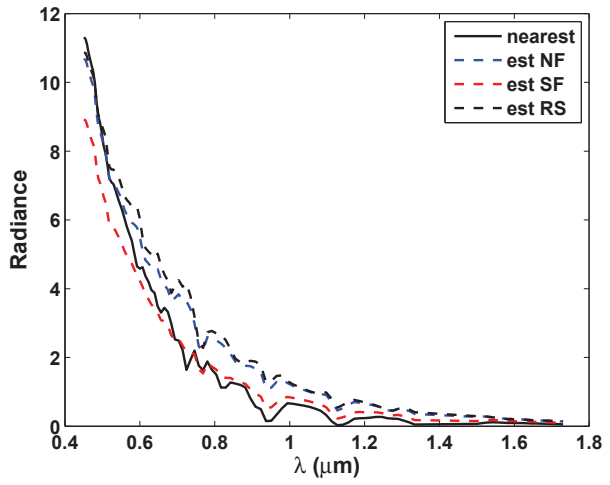

(c) $\mathbf{L}_{p}$

Figure 4.122: RT estimates for time-2 HYDICE data for different reflectance estimation modalities using $10 \%$ shadow initialization. 


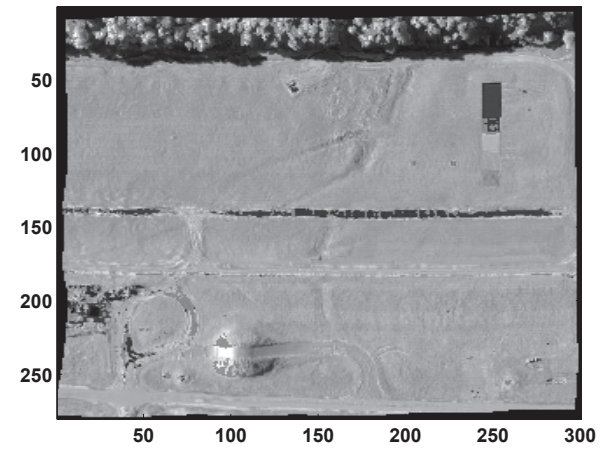

(a) $\hat{\tilde{\alpha}}^{(1)}$

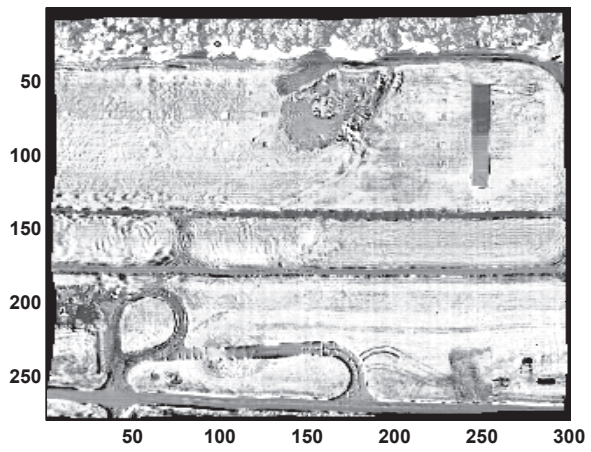

(b) $\hat{\beta}^{(1)}$

Figure 4.123: Estimated shadow coefficients for time-1 data using 10\% shadow initialization for HYDICE data

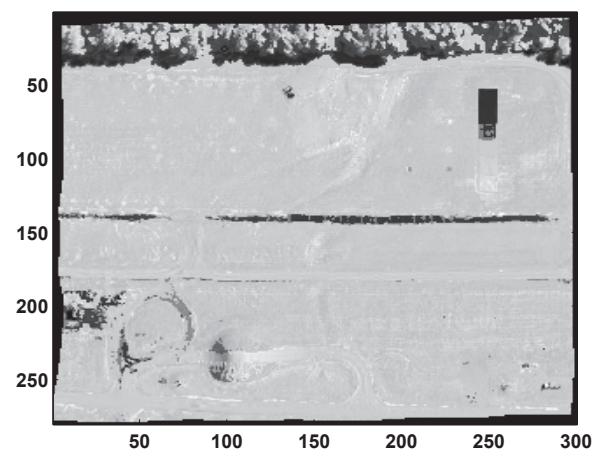

(a) $\hat{\tilde{\alpha}}^{(2)}$

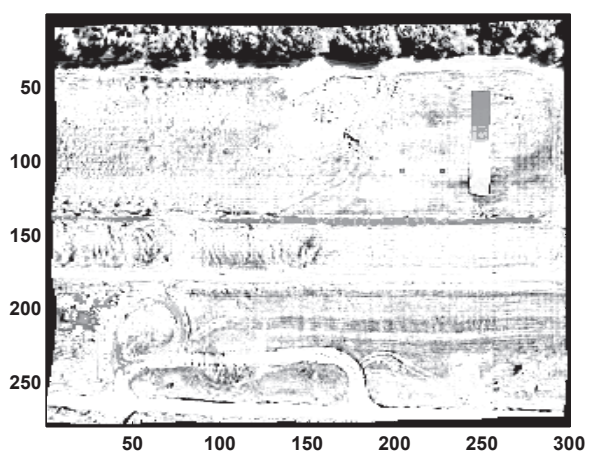

(b) $\hat{\beta}^{(2)}$

Figure 4.124: Estimated shadow coefficients for time-2 data using 10\% shadow initialization for HYDICE data 


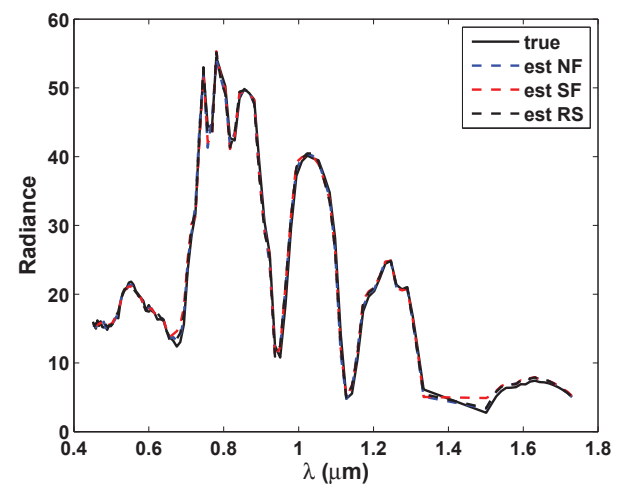

(a) Time-1

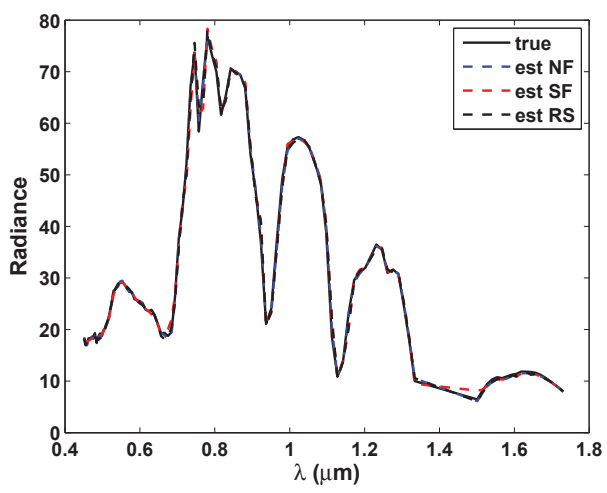

(b) Time-2

Figure 4.125: Comparison of estimated measurement $\hat{L}$ with true measurement $L$ for grass pixel using 10\% shadow initialization for HYDICE data

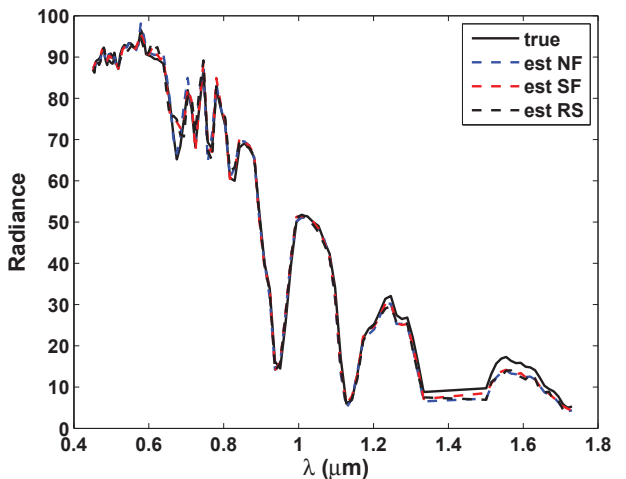

(a) Time-1

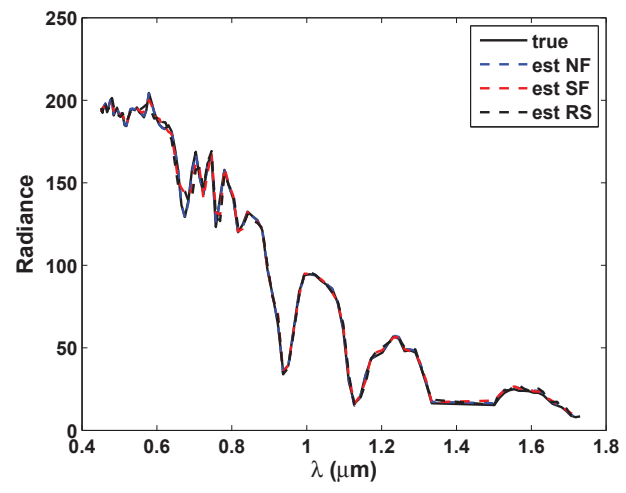

(b) Time-2

Figure 4.126: Comparison of estimated measurement $L$ with true measurement for $64 \%$ gray panel pixel using 10\% shadow initialization for HYDICE data 


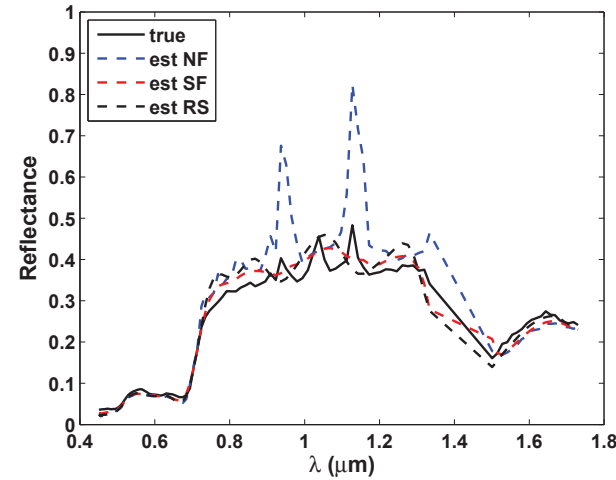

(a) Grass

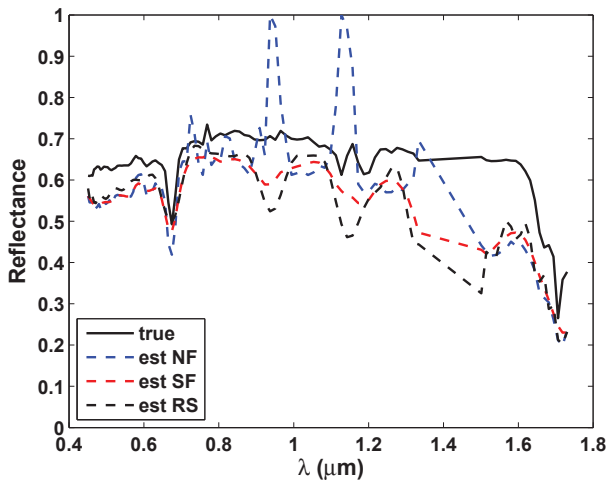

(b) $64 \%$ gray panel

Figure 4.127: Comparison of estimated reflectance $\rho$ with true reflectance for grass pixel and 64\% gray panel using 10\% shadow initialization for HYDICE data

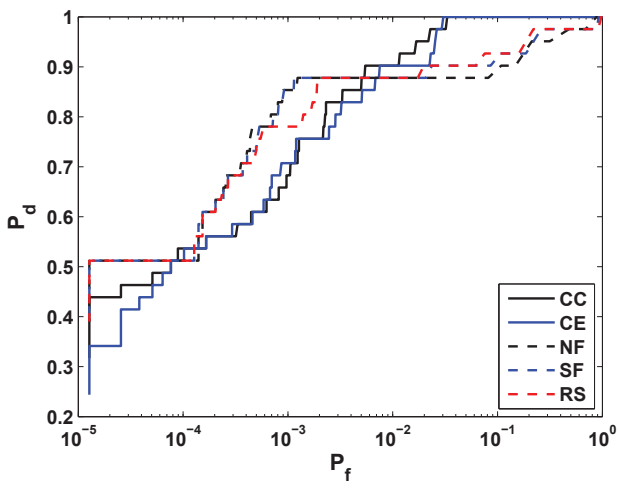

Figure 4.128: Empirical ROC curve performance for different reflectance estimation modalities using 10\% shadow initialization in comparison with $\mathrm{CC}$ and $\mathrm{CE}$ algorithms for HYDICE data 


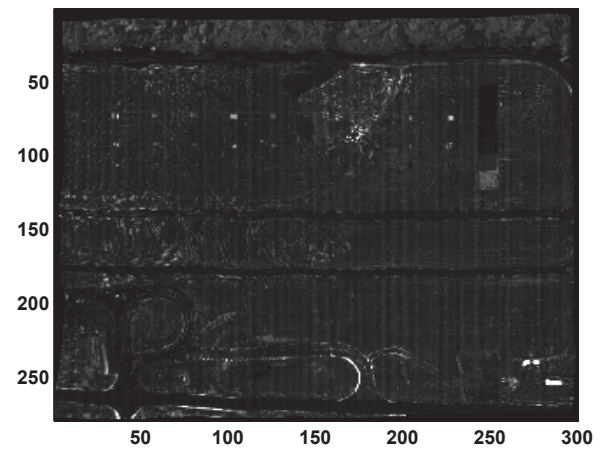

(a) No reflectance filtering

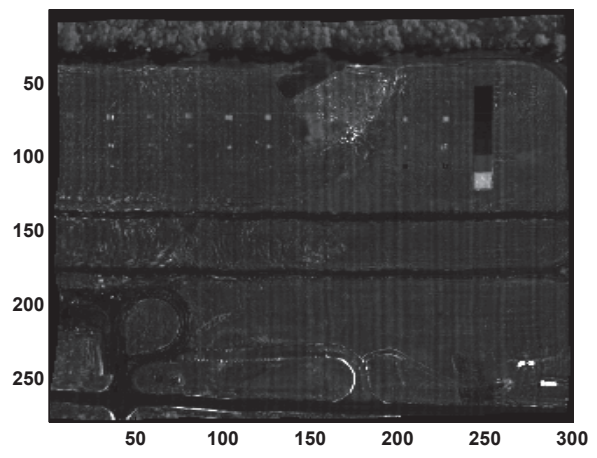

(b) Reflectance filtering

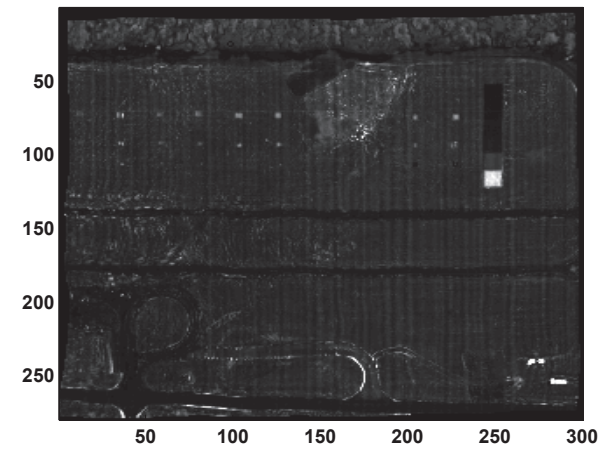

(c) Reflectance subspace

Figure 4.129: Comparison of detection statistic images for different MB reflectance modalities using $10 \%$ shadow initialization for HYDICE data 


\section{SCR Results}

Examination of the SCR statistics provides further insight into any subtle differences in detection performance that may exist for the two initialization cases. Figures 4.130 and 4.131 display the SCR for each target pixel in the scene for the different shadow initialization cases. Figure 4.132 displays the SCR results for CC and CE. The average SCR results are provided in Table 4.6. In general, the MB methods

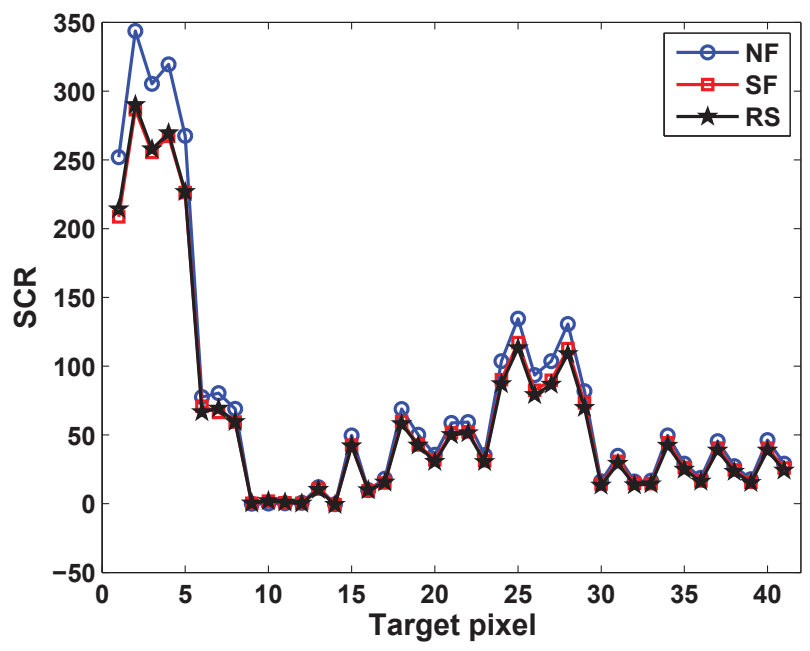

Figure 4.130: Comparison of SCR for different MB reflectance modalities using 5\% shadow initialization for HYDICE data

achieve a higher SCR than the $\mathrm{CC}$ and $\mathrm{CE}$ methods. Amongst the MB methods, the non-filtering (NF) method achieves the highest SCR and the SF and RS methods achieve similar performance. A slight decrease in SCR exists when using 10\% shadow initialization versus $5 \%$ initialization. 


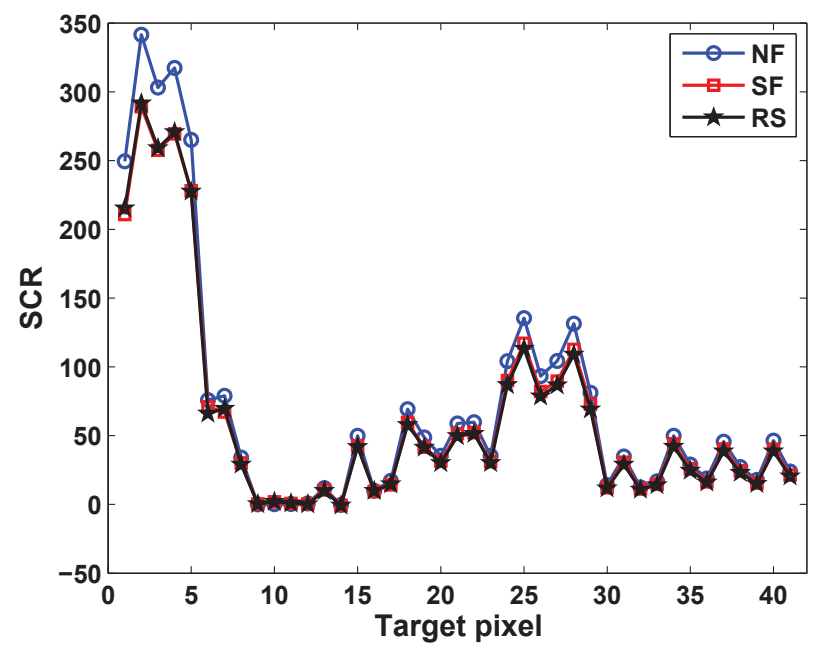

Figure 4.131: Comparison of SCR for different MB reflectance modalities using 10\% shadow initialization for HYDICE data

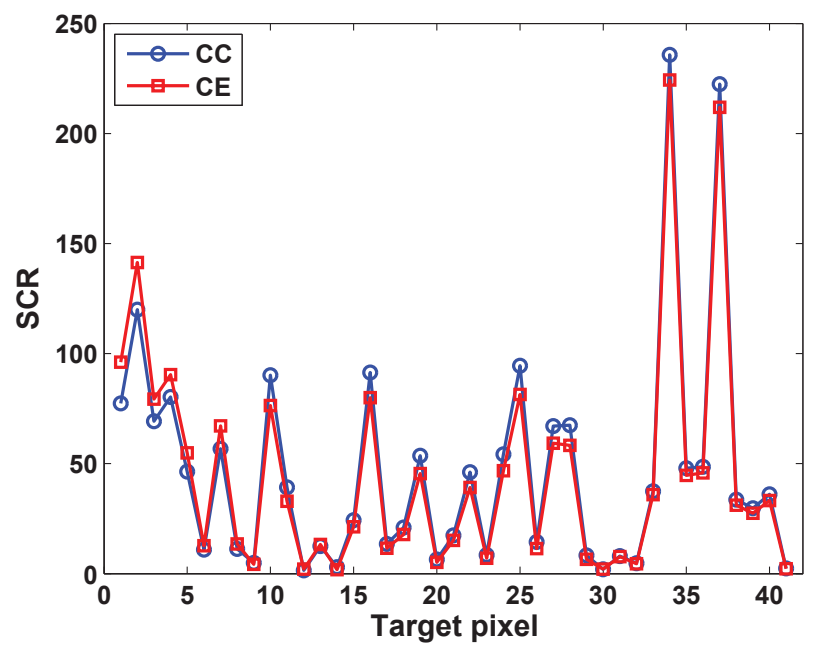

Figure 4.132: Comparison of SCR for CC and CE algorithms for HYDICE data 


\begin{tabular}{|c|c|c|c|c|c|}
\hline Algorithm & $\hat{\mu}_{t}$ & $\hat{\mu}_{b}$ & $\hat{\mu}_{t}-\hat{\mu}_{b}$ & $\hat{\sigma}_{b}$ & $\hat{\mu}_{S C R}$ \\
\hline CC & $1.11 \times 10^{3}$ & 9.46 & $1.10 \times 10^{3}$ & 23.55 & 46.83 \\
\hline CE & 947.32 & 9.54 & 937.78 & 20.63 & 45.44 \\
\hline NF-5\% & $1.30 \times 10^{4}$ & 140.11 & $1.28 \times 10^{4}$ & 169.26 & 75.83 \\
\hline NF-10\% & $1.26 \times 10^{4}$ & 141.82 & $1.24 \times 10^{4}$ & 167.80 & 74.36 \\
\hline SF-5\% & $1.33 \times 10^{4}$ & 257.35 & $1.30 \times 10^{4}$ & 200.64 & 64.98 \\
\hline SF-10\% & $1.30 \times 10^{4}$ & 256.31 & $1.27 \times 10^{4}$ & 199.35 & 64.16 \\
\hline RS-5\% & $1.36 \times 10^{4}$ & 232.22 & $1.33 \times 10^{4}$ & 208.22 & 64.25 \\
\hline RS-10\% & $1.33 \times 10^{4}$ & 231.79 & $1.30 \times 10^{4}$ & 206.60 & 63.33 \\
\hline
\end{tabular}

Table 4.6: Signal to clutter ratio for various algorithms using HYDICE data set

\subsubsection{Results using Relatively Calibrated Data Model}

Next, the 4-step alternating optimization algorithm using the relatively calibrated data model given in (2.84) is applied to the HYDICE change pair. As discussed earlier, the HYDICE data is calibrated to radiance units. Consequently, the relative gain and offset terms are expected to have values of $\mathbf{a}_{g}^{(t)}[m]=1$ and $\mathbf{b}_{g}^{(t)}[m]=0$. The shadow coefficients and basis coefficients are initialized as earlier when using the original physical model with $5 \%$ histogram threshold method. No spectral filtering is applied to the reflectance estimates.

Figures 4.133 and 4.134 display the final estimated relative gain and offset terms associated with the data model. For the most part, the relative gain values for both the time-1 and time-2 data linger around their expected values of 1 . The relative offset values, however, vary considerably from their expected value of 0 . This discrepancy may be related to the final estimated RT terms for the data or from model mismatch error. The time- 1 and time-2 estimates of the RT terms are given in Figures 4.135 and 4.136. In general, the estimated downwelling radiance using the relatively calibrated 


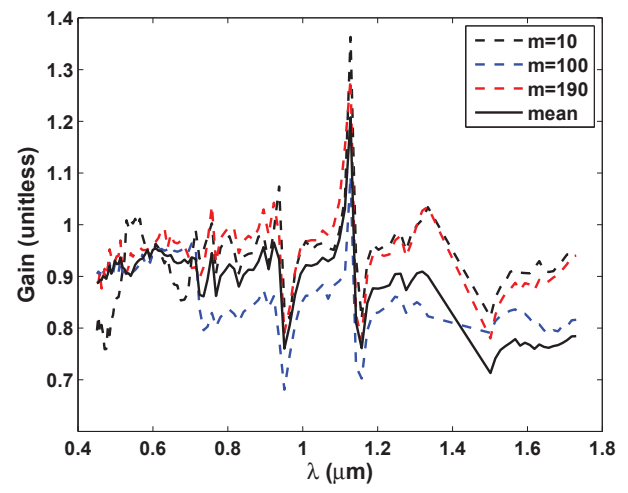

(a) Time-1

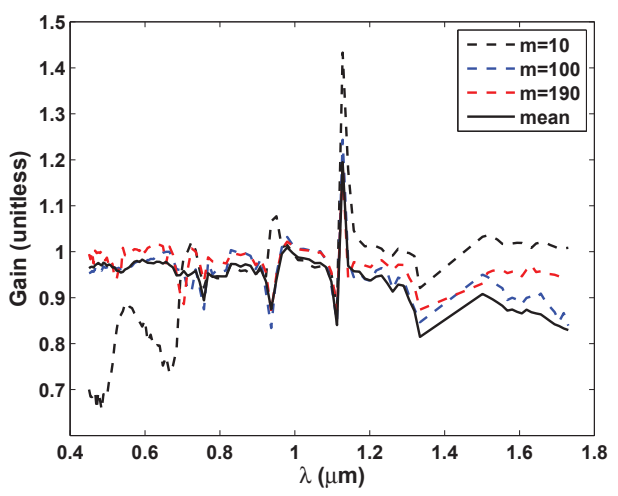

(b) Time-2

Figure 4.133: Estimated relative gain $\mathbf{a}_{g}^{(t)}$ for HYDICE data

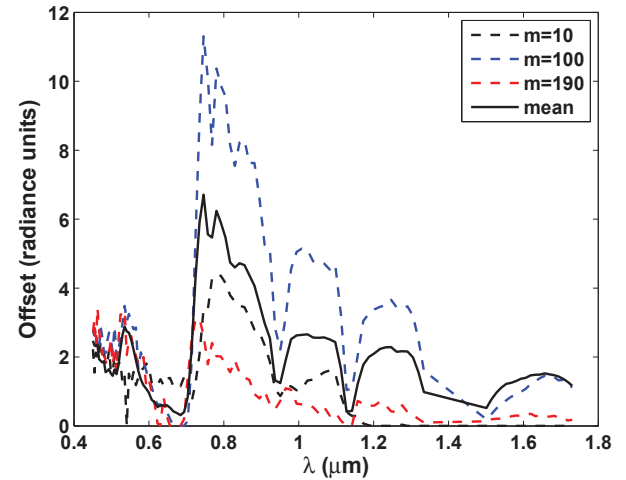

(a) Time-1

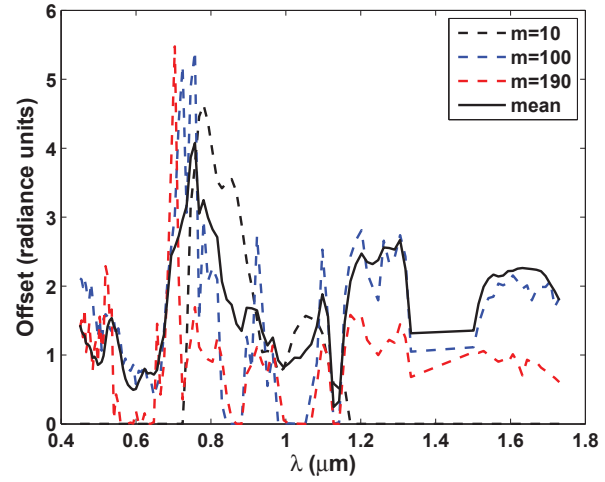

(b) Time-2

Figure 4.134: Estimated relative offset $\mathbf{b}_{g}^{(t)}$ for HYDICE data 


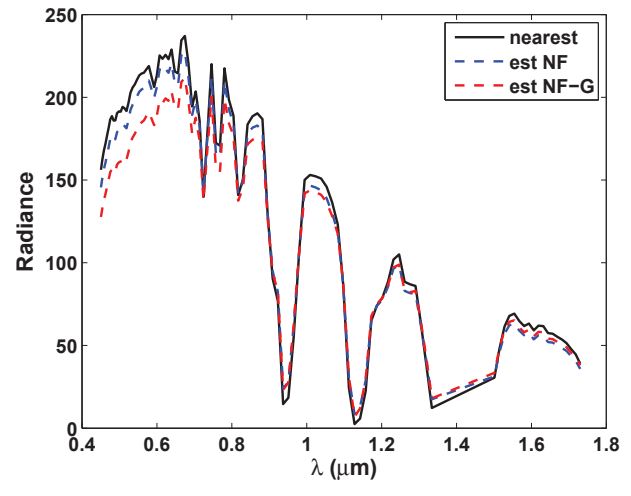

(a) $\boldsymbol{\tau} \odot \mathbf{L}_{s}$

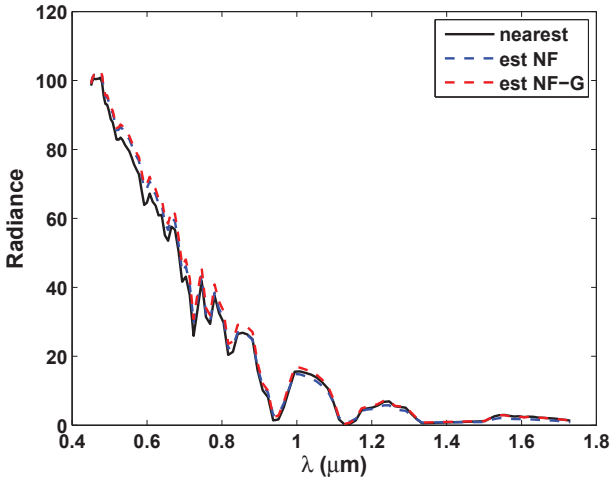

(b) $\boldsymbol{\tau} \odot \mathbf{L}_{d}$

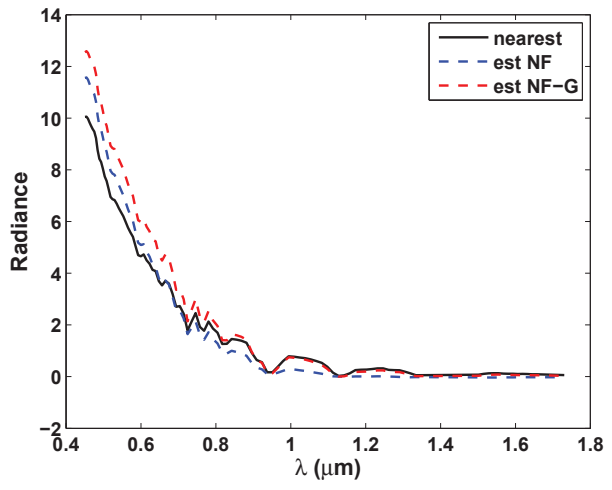

(c) $\mathbf{L}_{p}$

Figure 4.135: RT estimates for time-1 HYDICE data for both original data model and relative calibration model 


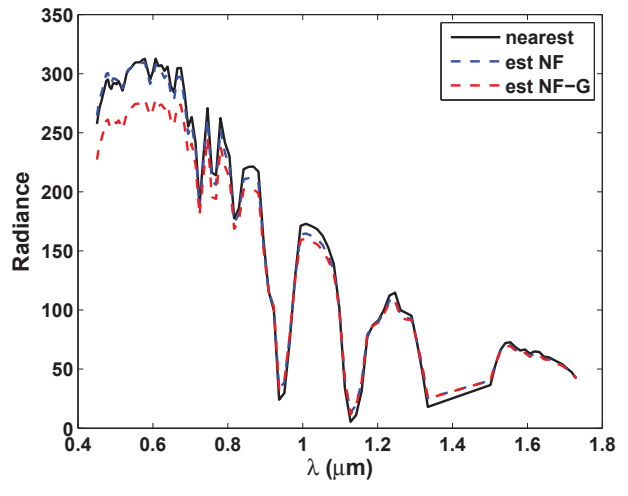

(a) $\boldsymbol{\tau} \odot \mathbf{L}_{s}$

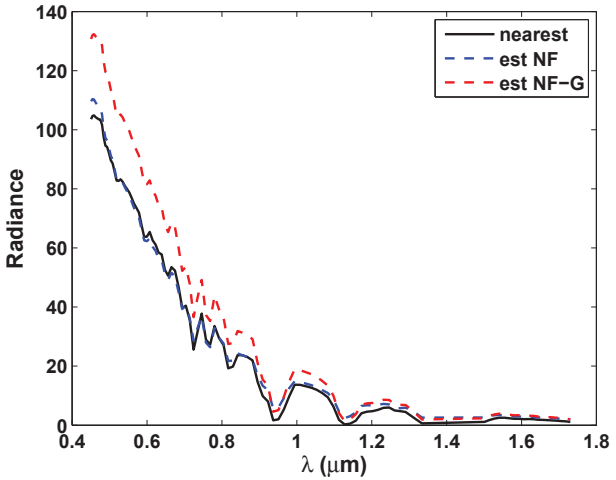

(b) $\boldsymbol{\tau} \odot \mathbf{L}_{d}$

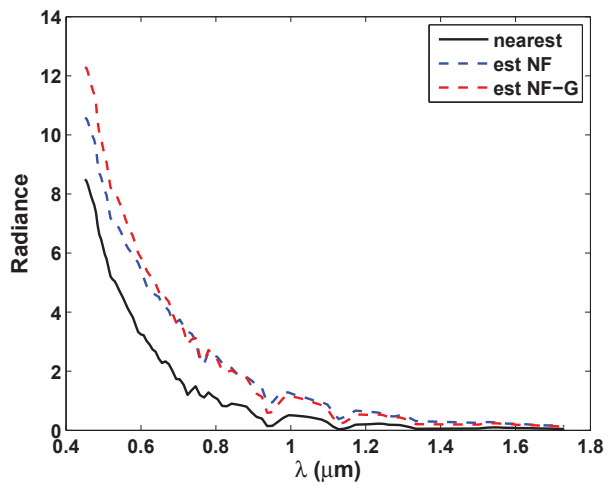

(c) $\mathbf{L}_{p}$

Figure 4.136: RT estimates for time-2 HYDICE data for both original data model and relative calibration model 
model is less than that obtained using the original data model. As a result, the calibration offset term may be compensating for the difference.

Figures 4.137 and 4.138 display the estimated shadow coefficients for this case. The estimates are similar to those obtained using the original data model and correlate well with the shadow conditions visible in the scene color images.

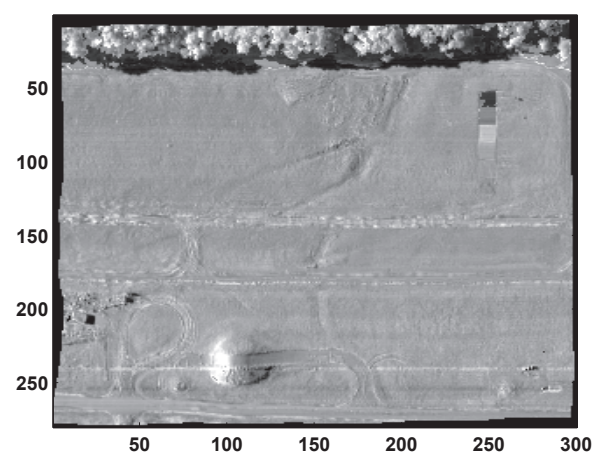

(a) $\hat{\tilde{\alpha}}^{(1)}$

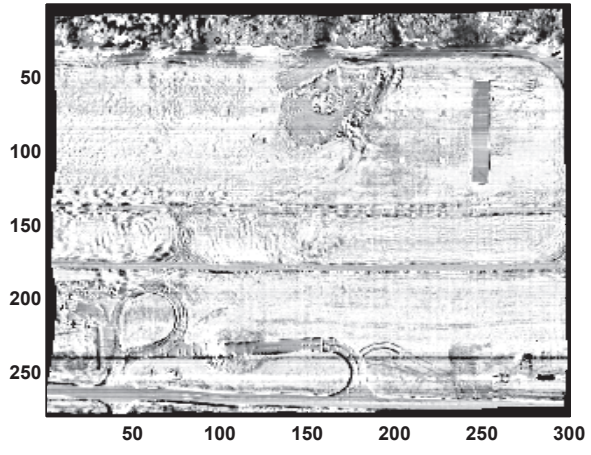

(b) $\hat{\beta}^{(1)}$

Figure 4.137: Estimated shadow coefficients for time-1 data using 5\% shadow initialization with relatively calibrated data model for HYDICE data

Figure 4.139 compares the ROC performance of the MB methods using the original data model $(\mathrm{NF})$ and the relatively calibrated data model (NF-G). The ROC performance decreases when using the general data model. Figure 4.140 displays the detection statistic images for both of the MB methods for further insight. The total background clutter is further reduced using the general model. However, this reduction comes at the expense of target suppression as well. The overall SCR for the original model is $S C R_{N F}=75.83$, whereas that of the general data model is 


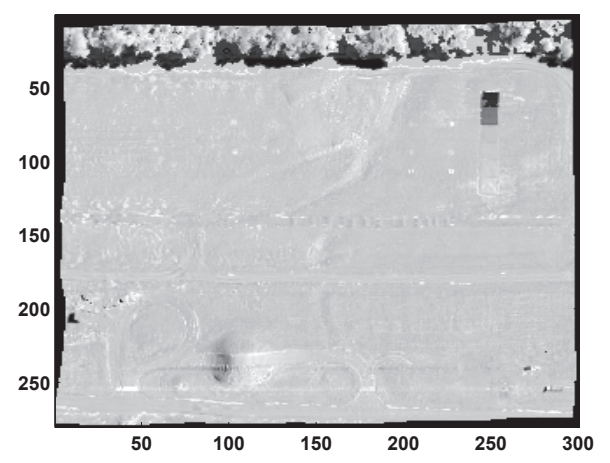

(a) $\hat{\tilde{\alpha}}^{(2)}$

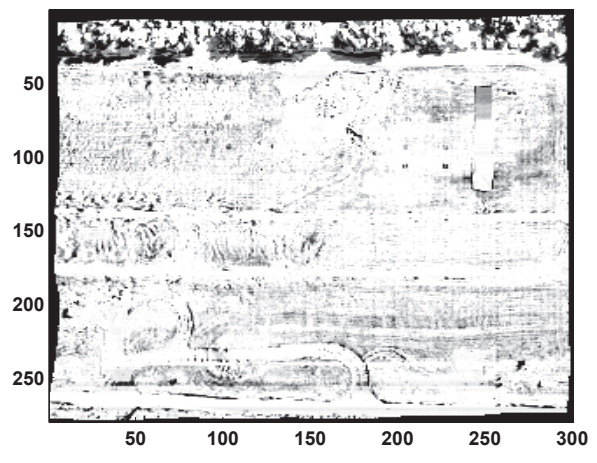

(b) $\hat{\beta}^{(2)}$

Figure 4.138: Estimated shadow coefficients for time-2 data using 5\% shadow initialization with relatively calibrated data model for HYDICE data

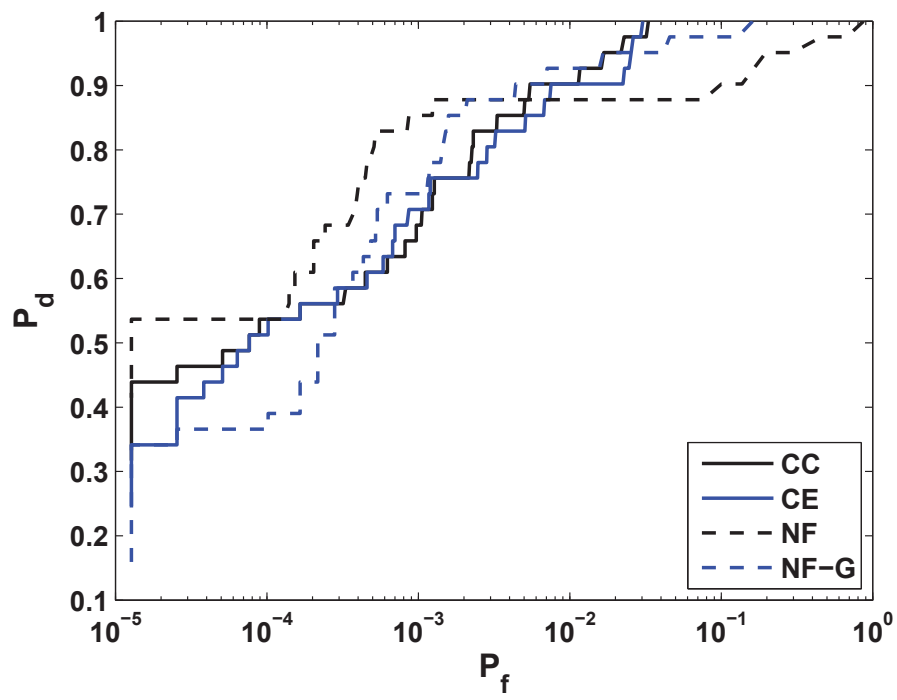

Figure 4.139: Empirical ROC curve performance for baseline MB method using original data model and relatively calibrated data model in comparison with $\mathrm{CC}$ and $\mathrm{CE}$ algorithms for HYDICE data 


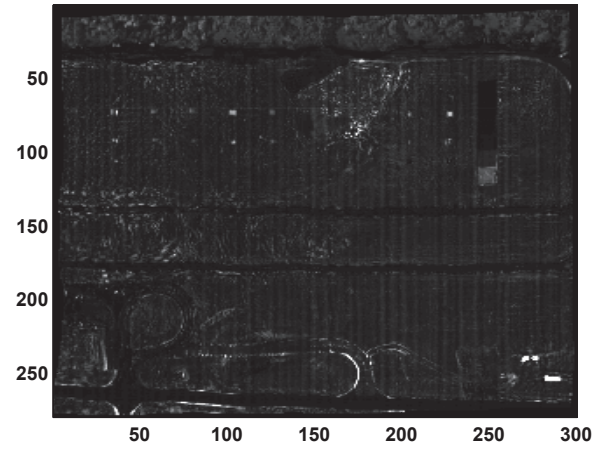

(a) Original data model

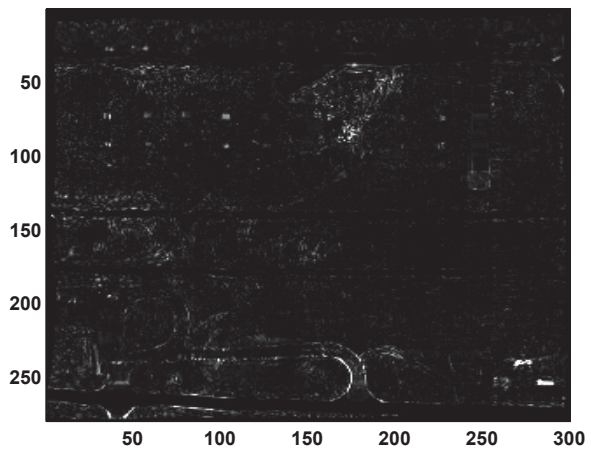

(b) Relative calibration data model

Figure 4.140: Comparison of detection statistic images for MB methods using original data model and relative calibration data model for HYDICE data

$S C R_{N F-G}=45.41$. While the added degrees of freedom help with overall background suppression, the same residual clutter areas exist as in the original data model (along the roads and for several of the fabric targets), which suggests that residual calibration error is not the cause of these false alarms.

\subsection{ARCHER Data}

Next, the MB change detection method is applied to ARCHER data. Application on data collected by this sensor is challenging due to calibration, geocorrection, and registration processes applied to the uncalibrated data sets. The relatively calibrated data model is used for this data set and the detection and estimation results are presented.

\subsubsection{Data Summary}

ARCHER is a VNIR pushbroom hyperspectral system flown by the Civil Air Patrol (CAP) [81]. Table 4.7 displays the specifications of the sensor . The hyperspectral 


\begin{tabular}{|l|c|}
\hline Platform: & Gippsland GA-8 \\
\hline Altitude: & $762 \mathrm{~m}(2500 \mathrm{ft})$ AGL \\
\hline Ground Swath: & $0.5 \mathrm{~km}$ \\
\hline Wavelength range: & $0.5-1.0 \mu \mathrm{m}$ \\
\hline Spectral bands: & 40 \\
\hline Spectral Resolution: & $11.7 \mathrm{~nm}(\mathrm{avg})$ \\
\hline GSD & $1 \mathrm{~m}$ \\
\hline
\end{tabular}

Table 4.7: ARCHER sensor information

data cubes possess a wavelength range of $0.5-1.0 \mu m$ with $K=40$ spectral bands with an average spectral resolution of $11.7 \mathrm{~nm}$.

The uncalibrated imagery collected by the system must be calibrated to remove detector non-uniformity, geocorrected to remove image warping resulting from aircraft rolling during collection, and co-registered to produce a change pair. The system lacks an on-board calibration source to perform NUC. Consequently, the calibration process first removes an estimate of the dark current and then utilizes an in-scene method to remove sensor gain nonuniformity. The in-scene NUC method is applied adaptively over the scene by estimating the spectral mean in the along-track spatial dimension for a relatively large number of samples. One of the rows in the along-track dimension is chosen as the reference and the NUC gain is applied to the remaining rows such that the spectral mean of the other rows is the same as the reference row. The spectral mean statistics are re-estimated at regular intervals after a certain number of samples are collected. The implicit assumption for this approach is that within the samples averaged, the pixels should be covering similar material classes on the ground. Consequently, the long-term spatial averages within the block should be approximately equal and can be used to estimate the amount of detector response 
non-uniformity. However, this approach is very susceptible to anomalous pixels within the block, which can skew the mean statistics along a row.

The data used for this study were collected at Ft. A.P. Hill $\left(38^{\circ} 7^{\prime} 10^{\prime \prime} N, 77^{\circ} 16^{\prime} 1^{\prime \prime} W\right)$, which has a nominal altitude near 66m (216.5ft) ASL, as part of the Multisensor Architecture Spectral Test (MAST) campaign. Figure 4.141 displays color images of the two data sets, collected June 15, 2005 and June 17, 2005 respectively, with change targets identified. The scene is comprised of a mostly natural vegetative background

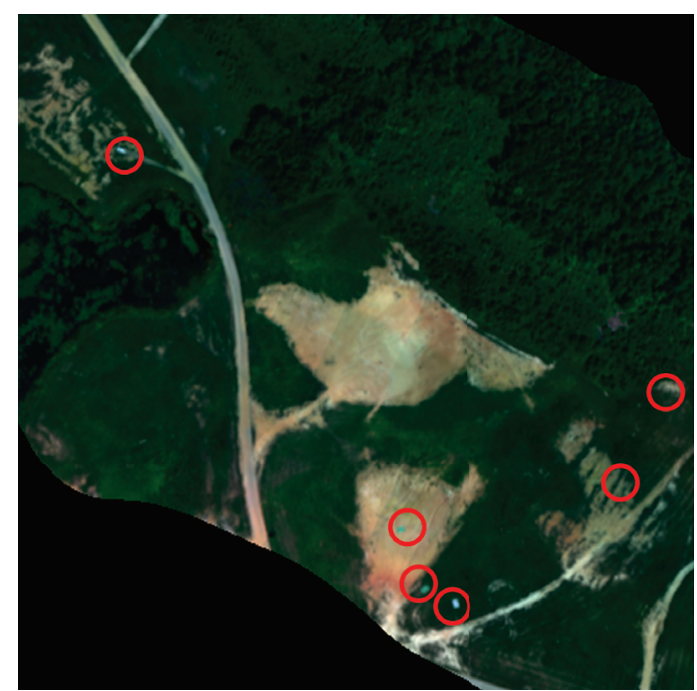

(a) June 15, 2005

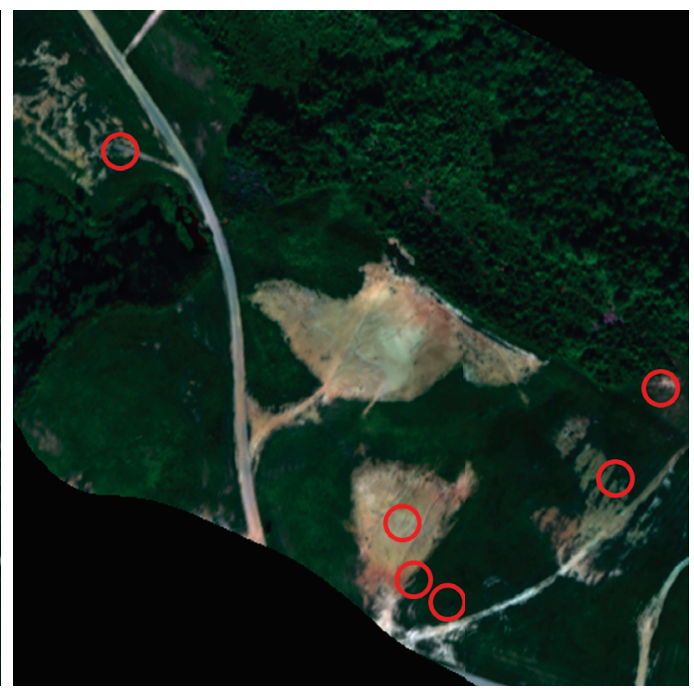

(b) June 17, 2005

Figure 4.141: ARCHER MAST change pair

with several roads/paths and large dirt patches. The change targets correspond to various tarps and nets removed/inserted between the two scenes. Spectral ground truth for objects in the scene are not available. Both of the data sets were collected in the morning of the respective day. However, the data are collected nearly two hours apart, which results in significant shadow variation between the two images. 
The shadow differences are apparent along the edges of the tree regions in the scene. This collection information is provided in Table 4.8. Due to a lack of calibration data

\begin{tabular}{|c|c|c|c|}
\hline Date & Acquisition Time (EDT) & SE & SA \\
\hline June 15,2005 & $10: 49$ & $56.64^{\circ}$ & $106.03^{\circ}$ \\
\hline June 17,2005 & $09: 09$ & $37.13^{\circ}$ & $87.75^{\circ}$ \\
\hline
\end{tabular}

Table 4.8: ARCHER MAST data collection information

and a lack of spatially uniform regions in the scene, an accurate noise estimate cannot be obtained for this data. Consequently, a white noise model is applied for the MB change detection method.

\subsubsection{Results using Relatively Calibrated Data Model}

Analysis of results for the other data sets tested to this point demonstrate a minimal dependence for detection performance on the shadow initialization used. For this data set, the residual calibration error is of greater importance. As discussed, a relative calibration procedure is applied to the data. Consequently, the relatively calibrated data model given in (2.84) must be applied to this change pair. Due to the geocorrection and registration applied to these data sets, the original spatial dependence of the relative gain $\mathbf{a}_{g}^{(t)}$ and offset $\mathbf{b}_{g}^{(t)}$ has been warped and is no longer simply dependent on $m$. The spatial dependence is more complicated and unknown for this data. As a result, two methods are applied for estimation of the relative gain and offset terms. In the first method, the gain and offset are simply assumed spatially constant across the whole scene, i.e. not dependent on $m$ or $n$. In the second method, an adaptive approach is utilized for which the gain and offset are assumed spatially 
constant within a small block of size $B_{m} \times B_{n}$. The image is divided into a total of $B$ blocks of this size (except for image boundaries where the block size will be smaller) and the gain and offset are estimated separately for each block. This approach more closely resembles the adaptive relative in-scene calibration technique applied to these data.

For both techniques, the RT terms are initialized from MODTRAN using a standard mid-latitude summer model with $20 \mathrm{~km}$ visibility and the default water vapor column along with the known sensor altitude and solar position associated with each data cube. The shadow coefficients are initialized using the bottom $5 \%$ of spectral norm pixels as shadow pixels with $\alpha=0.1$ and $\beta=0.5$. The cosine projection term is initialized as $\cos \theta_{s}^{(t)}$ using the known solar zenith $\theta_{s}$ for both time-1 and time- 2 . Figures 4.142 and 4.143 display the initial estimates of the time- 1 and time- 2 shadow coefficients. The detected shadow pixels include shadowed areas along the treeline

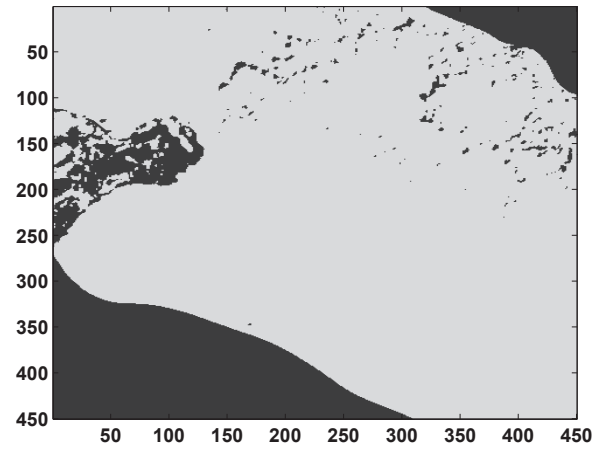

(a) $\tilde{\alpha}^{(1)}$

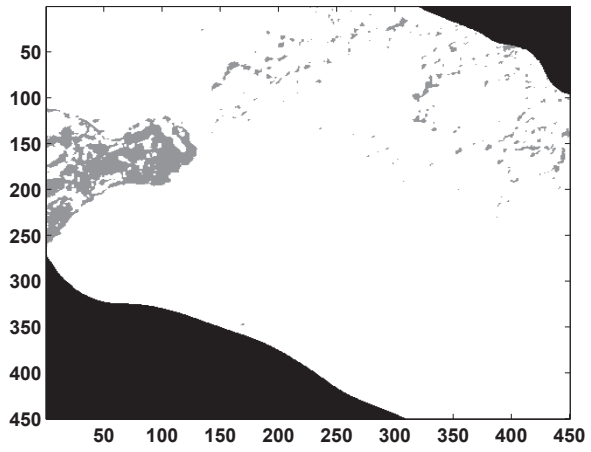

(b) $\beta^{(1)}$

Figure 4.142: Initial estimates for time-1 shadow coefficients using bottom $5 \%$ of spectral norm pixels for MAST data 


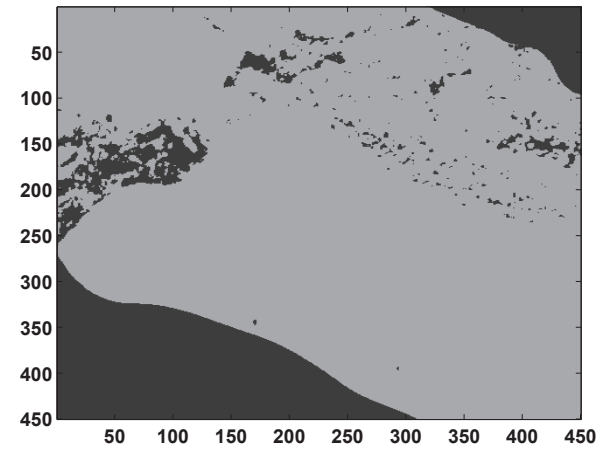

(a) $\tilde{\alpha}^{(2)}$

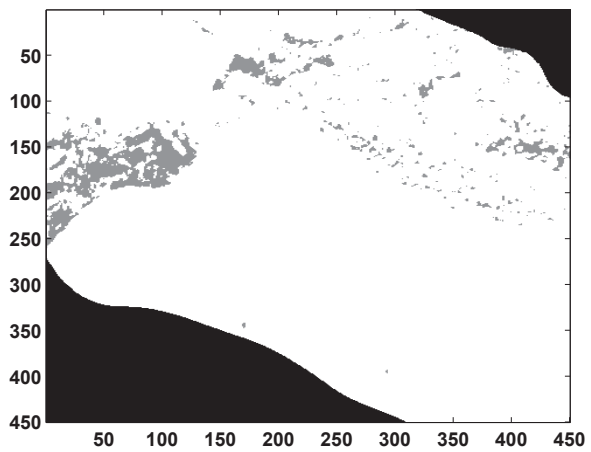

(b) $\beta^{(2)}$

Figure 4.143: Initial estimates for time-2 shadow coefficients using bottom $10 \%$ of spectral norm pixels for MAST data

and also a darker area in the bottom left portion of the scene that may be water.

The relative calibration gain and offset terms are initialized using the NDVI method previously described using the general leaf reflectance signature provided in Figure 4.144 as the vegetation signature. The initial gain and offset using this technique are shown in Figure 4.145. Due to dark current subtraction, one expects the offset term to be near 0 . Deviation from this expected value can result from poor dark current subtraction or more likely from poor estimates of the shadow term or vegetation reflectance used for NDVI. These initial estimates are applied for all pixels for both the spatially constant case and for all blocks in the spatially adaptive case.

The ARCHER data is only 40 bands and has rather low spectral resolution. As a result, the spectral smoothing techniques employed for the reflectance estimates do not impact the results significantly. As a result, only the results using the baseline non-spectral-filtered reflectance estimates are shown here. The block size for the adaptive technique is $B_{m}=45$ by $B_{n}=45$ pixels. In general, the total number 


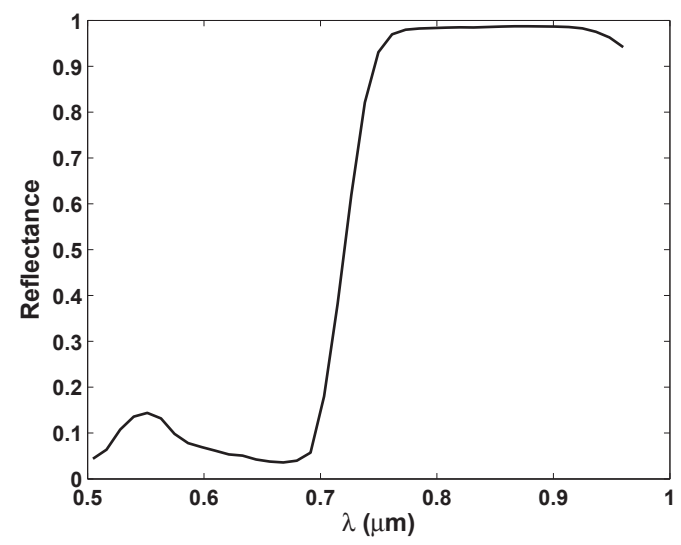

Figure 4.144: General leaf reflectance spectrum used to obtain initial calibration term estimates using NDVI for MAST data

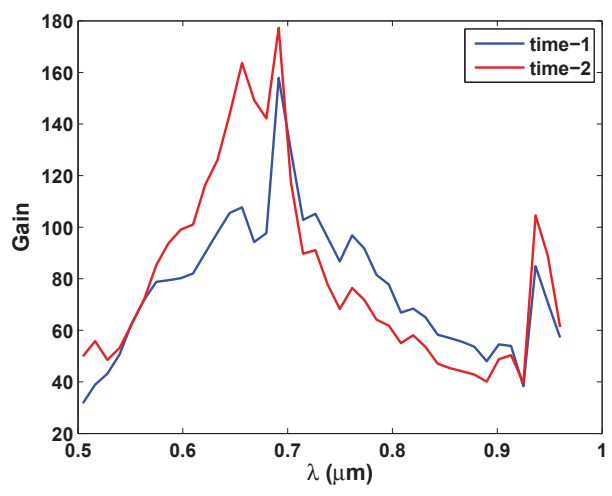

(a) Gain $\mathbf{a}_{g}^{(t)}$

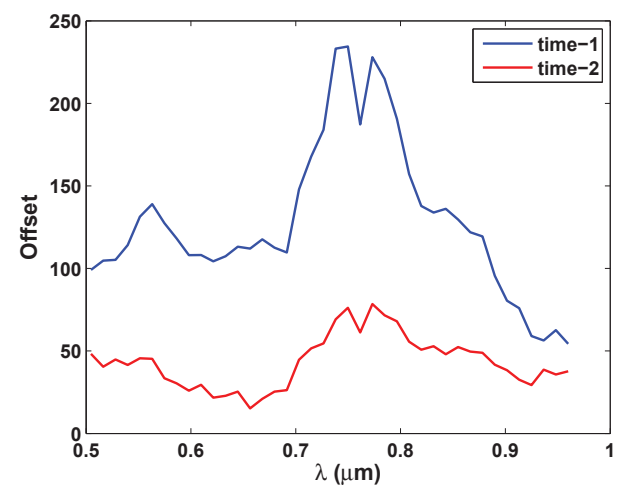

(b) Offset $\mathbf{b}_{g}^{(t)}$

Figure 4.145: Initial estimates of relative calibration gain and offset for ARCHER MAST data 
of pixels within each block must be greater than $2 K$ to avoid an under-determined estimation problem.

\section{Estimation Results}

Due to a lack of truth for the radiative transfer terms and the shadow coefficients, the accuracy of the estimates cannot be quantified. The results are simply provided here to ensure the results are reasonable. Figures 4.146 and 4.147 display the estimated shadow coefficients using spatially constant calibration terms for the data model. Figures 4.148 and 4.149 display the estimated shadow coefficients using spa-

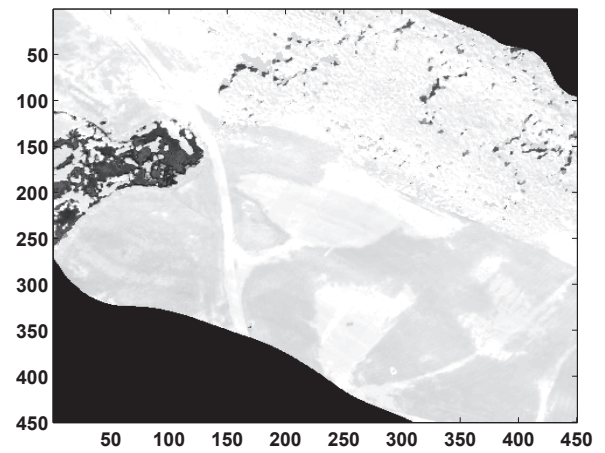

(a) $\hat{\tilde{\alpha}}^{(1)}$

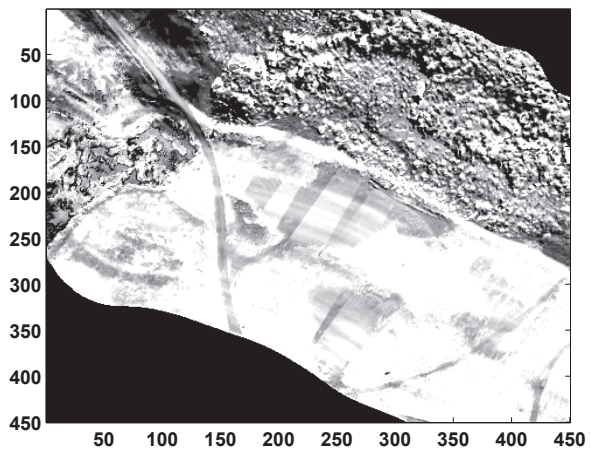

(b) $\hat{\beta}^{(1)}$

Figure 4.146: Estimated shadow coefficients for time-1 data using spatially constant calibration terms for MAST data

tially adaptive calibration terms for the data model. In general, the direct shadow coefficient estimates demonstrate correlation with the shadows visible in the color images of Figure 4.141. However, the indirect shadow coefficient estimates demonstrate unexpected artifacts that most likely result from compensation of residual calibration error or poor calibration term estimates. Additionally, discontinuities are visible in 


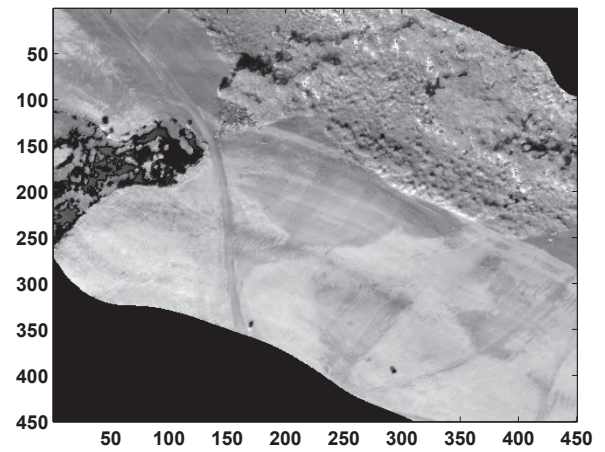

(a) $\hat{\tilde{\alpha}}^{(2)}$

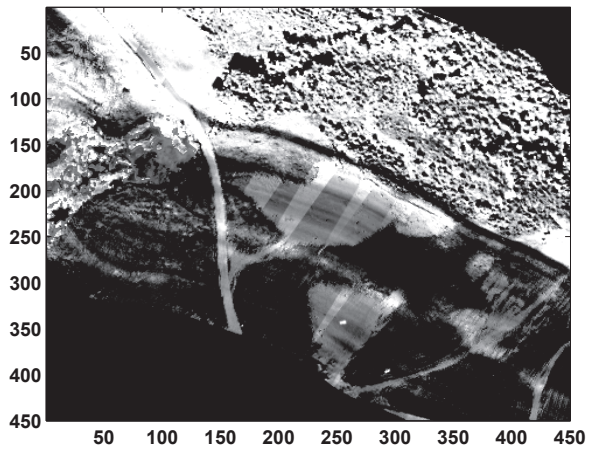

(b) $\hat{\beta}^{(2)}$

Figure 4.147: Estimated shadow coefficients for time-2 data using spatially constant calibration terms for MAST data

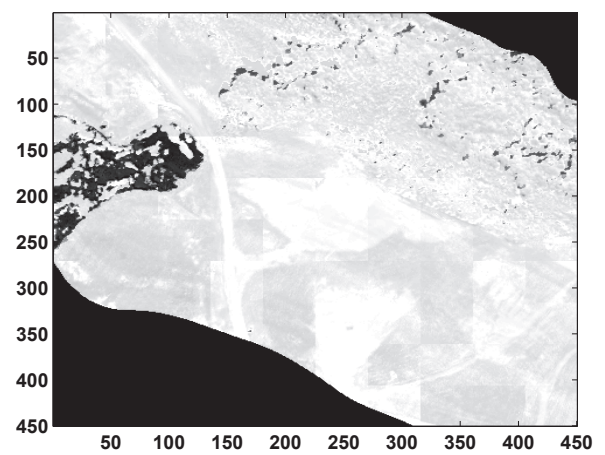

(a) $\hat{\tilde{\alpha}}^{(1)}$

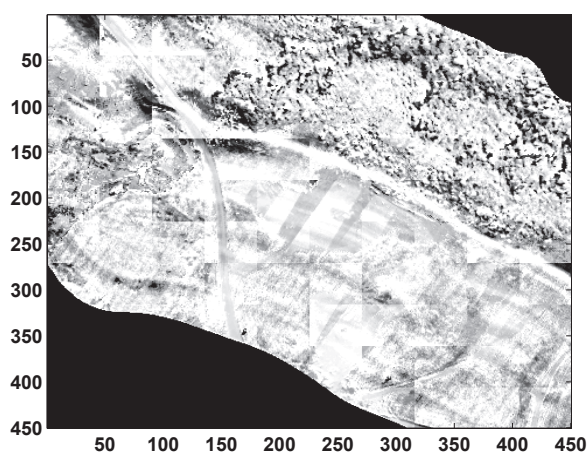

(b) $\hat{\beta}^{(1)}$

Figure 4.148: Estimated shadow coefficients for time-1 data using spatially adaptive calibration terms for MAST data 


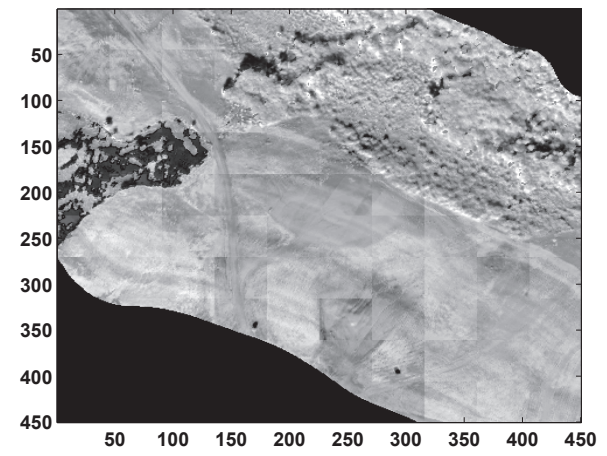

(a) $\hat{\tilde{\alpha}}^{(2)}$

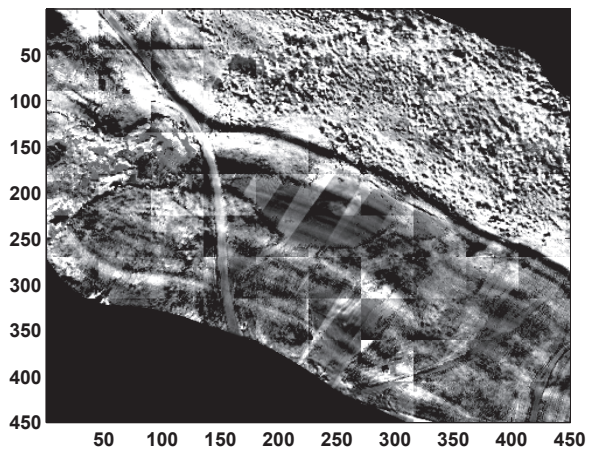

(b) $\hat{\beta}^{(2)}$

Figure 4.149: Estimated shadow coefficients for time-2 data using spatially adaptive calibration terms for MAST data

the shadow coefficient estimates for the adaptive method at the boundaries of each adaptive spatial block.

Figures 4.150 and 4.151 display the time-1 and time-2 RT estimates respectively. In general, the RT estimates for both methods are very similar for the time-1 data. The spatially adaptive method produces a higher direct solar radiance estimate and lower diffuse solar radiance and path radiance estimates for the time- 2 data.

Figure 4.152 displays the estimated calibration gain and offset using both the spatially constant calibration and the spatially adaptive calibration. The mean calibration gain and offset over all the adaptive blocks is shown here. The estimated calibration gain terms have a similar spectral shape as the mean detector gain for the AFRL data shown in Figure 4.89. The shape of the gain term is directly related to the spectral responsivity of the focal plane detector material. The AFRL camera and the ARCHER camera both use silicon detectors. As a result, the spectral responsivity and corresponding calibration gain are expected to be similar in shape. The estimated 


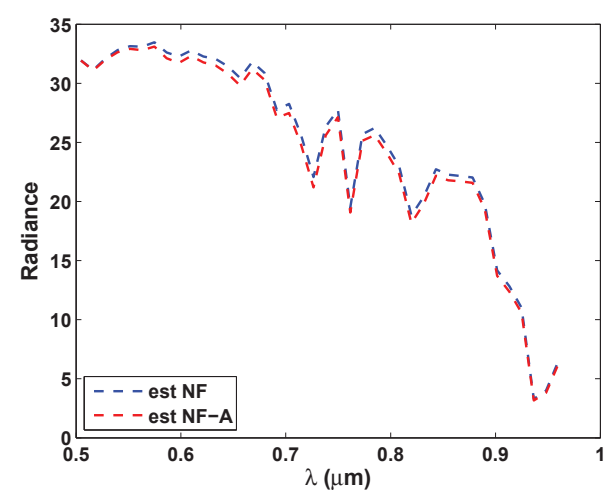

(a) $\boldsymbol{\tau} \odot \mathbf{L}_{s}$

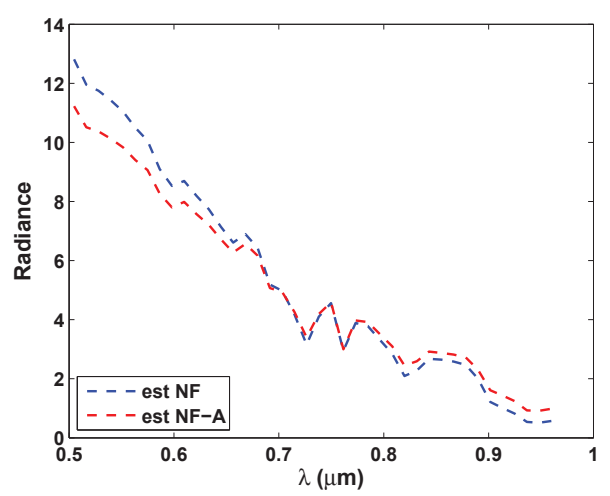

(b) $\boldsymbol{\tau} \odot \mathbf{L}_{d}$

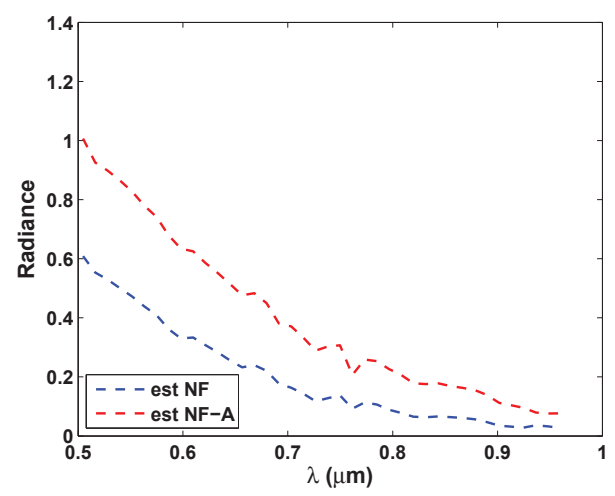

(c) $\mathbf{L}_{p}$

Figure 4.150: RT estimates for time-1 MAST data for both spatially constant (NF) and spatially adaptive (NF-A) calibration terms 


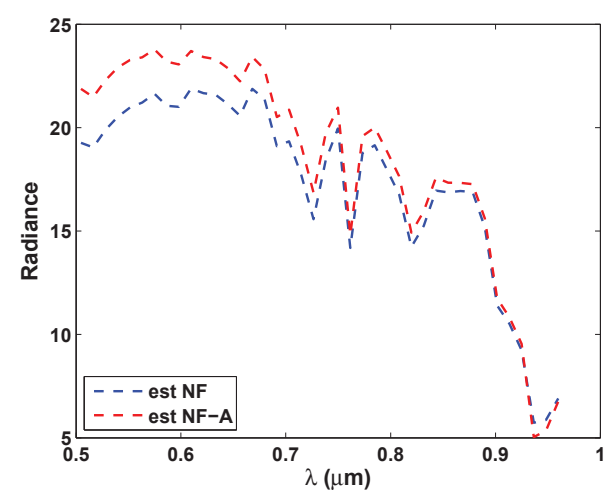

(a) $\boldsymbol{\tau} \odot \mathbf{L}_{s}$

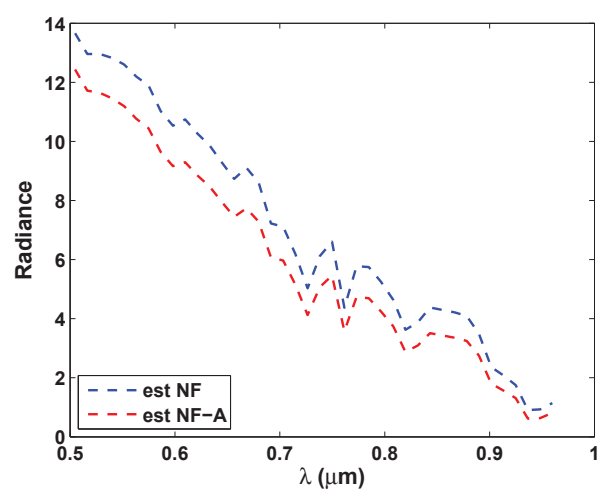

(b) $\boldsymbol{\tau} \odot \mathbf{L}_{d}$

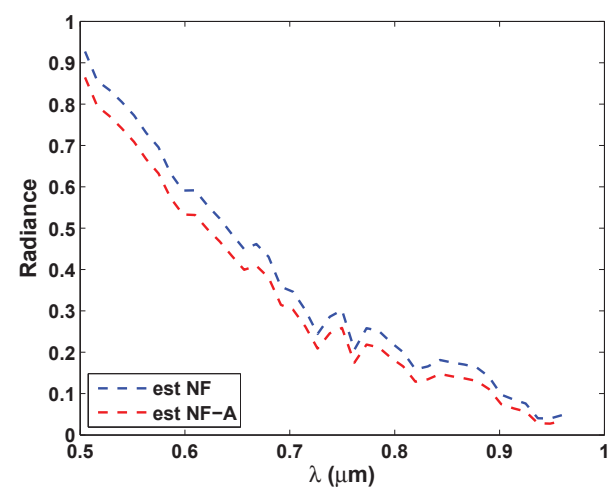

(c) $\mathbf{L}_{p}$

Figure 4.151: RT estimates for time-2 MAST data for both spatially constant (NF) and spatially adaptive (NF-A) calibration terms 


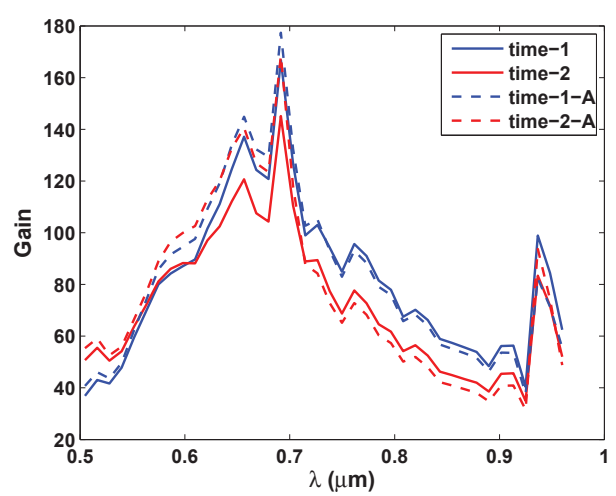

(a) Gain $\mathbf{a}_{g}^{(t)}$

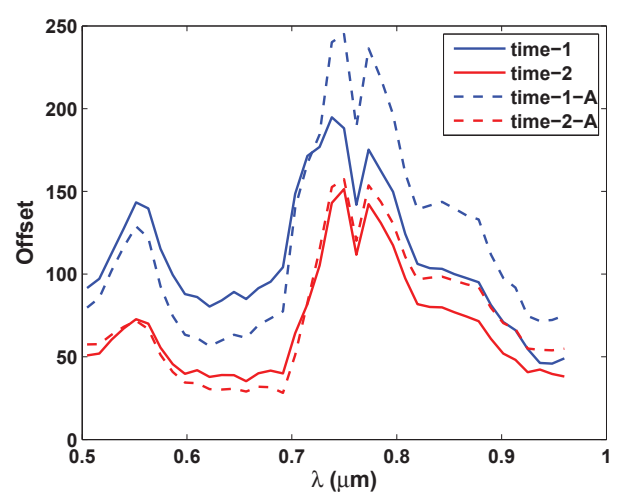

(b) Offset $\mathbf{b}_{g}^{(t)}$

Figure 4.152: Final estimates of relative calibration gain and offset for ARCHER MAST data using spatial constant calibration terms and spatially adaptive calibration terms (A). For the spatially adaptive case, the mean over all spatial blocks is plotted.

calibration offset terms demonstrate a spectral shape similar to that associated with vegetation pixels. A similar characteristic was seen for the AFRL data, where it was discussed that the calibration offset term may be compensating for adjacency effects not implicitly modeled by the original data model. Since the majority of the scene is vegetation in this case, the same occurrence may be happening here as well.

Figure 4.153 displays the estimated measurement and estimated reflectance associated with a grass pixel. The estimated grass reflectance is compared with a standard grass laboratory spectrum, which may not be representative of the true grass spectrum of the scene. However, the estimated reflectance does possess the same spectral shape associated with grass and vegetation in general. Additionally, the reflectance estimates are spectrally smooth, even without employing the spectral smoothing techniques associated with the alternative MB modalities. 


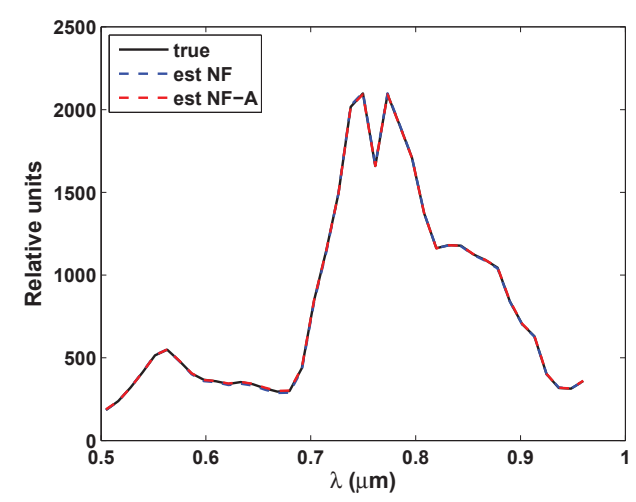

(a) Comparison of estimated measurement $\hat{L}$ with true measurement $L$

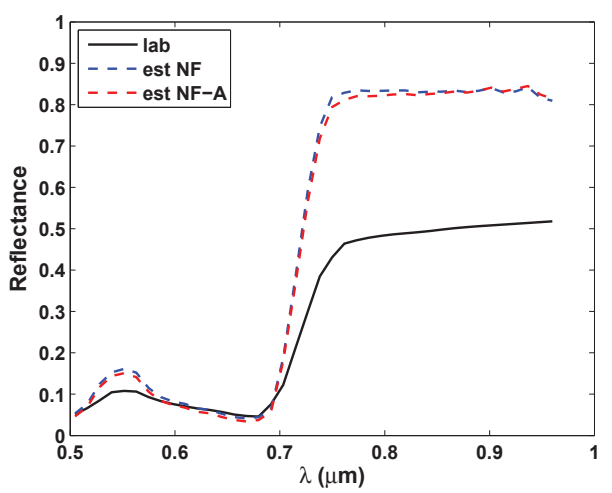

(b) Estimated reflectance

Figure 4.153: Estimation results for a grass pixel for MAST data using spatially constant calibration terms and spatially adaptive calibration terms

\subsubsection{Detection Results}

The detection results achieved using the MB method for the ARCHER data are compared with those achieved using the baseline CC and CE algorithms. Figure 4.154 displays the detection statistic images using the $\mathrm{CC}$ and $\mathrm{CE}$ detectors. The dynamic range of the images is stretched between 0 and $\mu+7 \sigma$.

While the majority of change targets readily appear in the detection statistic images for both $\mathrm{CC}$ and $\mathrm{CE}$, there does exist residual clutter leakage. Specifically, the majority of clutter leakage exists along the roads, the dirt patch, and in shadow regions near the treelines. Figure 4.155 displays the detection statistic images for the MB method using spatially constant and spatially adaptive calibration terms. Both of the MB methods demonstrate significant clutter leakage in comparison with the CC and CE algorithms. This clutter leakage most likely results from residual calibration error resulting from the in-scene NUC applied, the inability to properly estimate these 


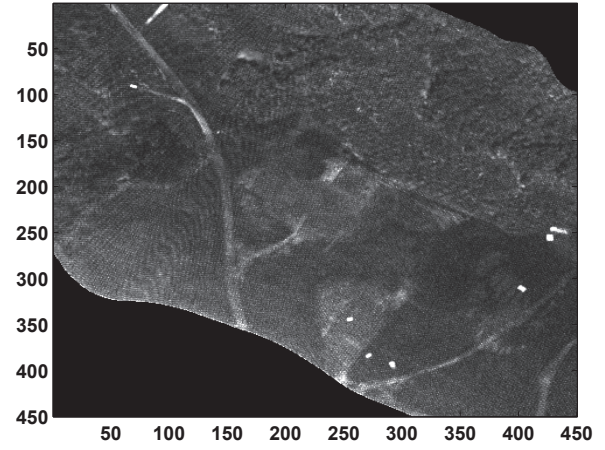

(a) $\mathrm{CC}$

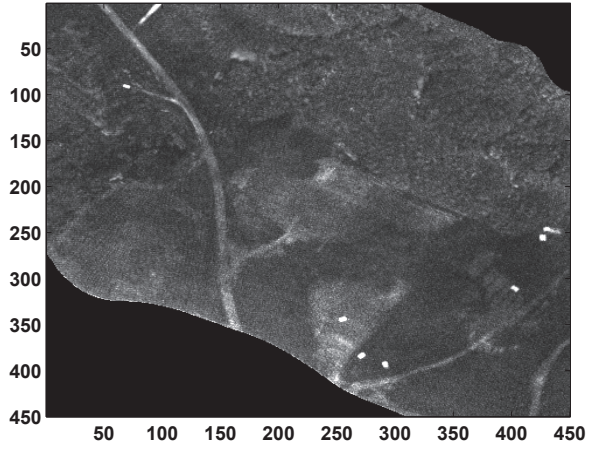

(b) $\mathrm{CE}$

Figure 4.154: Comparison of detection statistic images for CC and CE change detection algorithms for MAST data

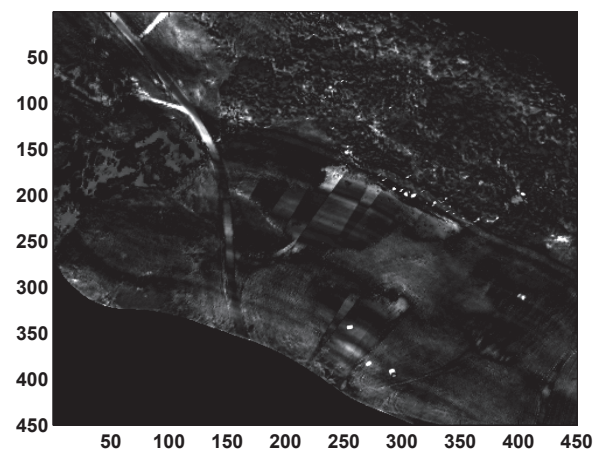

(a) Spatially constant calibration

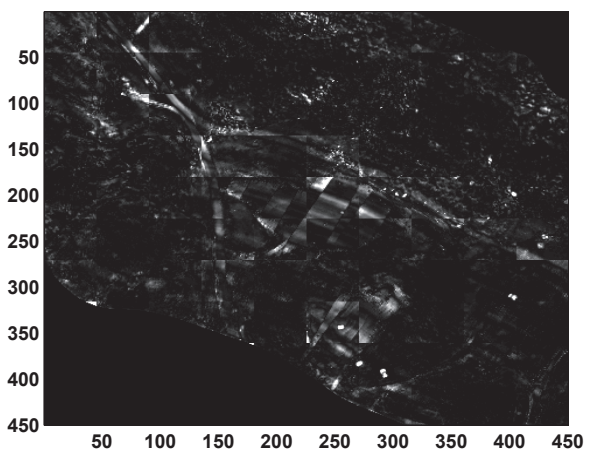

(b) Spatially adaptive calibration

Figure 4.155: Comparison of detection statistic images for MB method on MAST data using relative calibration data model 
calibration terms, and model mismatch error. Strong discontinuities are visible in the clutter leakage of these detection statistic images, which correspond to the adaptive inscene NUC applied to original data. Figure 4.156 displays the ROC curve performance associated with these detection statistic images. As expected from the detection

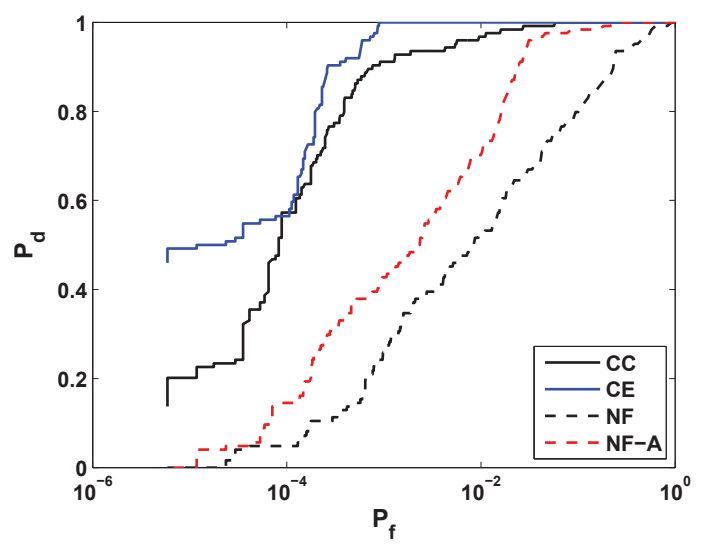

Figure 4.156: Empirical ROC curve performance for MB methods using relatively calibrated data model in comparison with $\mathrm{CC}$ and $\mathrm{CE}$ algorithms for MAST data

statistic images, the $\mathrm{CC}$ and $\mathrm{CE}$ algorithms vastly outperform the MB approaches for this data set. The CE algorithm achieves the highest performance of the group. The spatially adaptive calibration technique does improve ROC performance for the MB method but not to the level of performance associated with CC and CE. Table 4.9 compares the SCR performance of these algorithms, which further supports the ROC results. The spatially adaptive calibration technique reduces the standard deviation of the background by nearly a factor of 3, which results in the improved ROC and SCR performance. With $K=40$, the expected mean and variance of the MB detection statistic for the background are $\mu_{b}=2 K=80$ and $\sigma_{b}^{2}=4 K=160$ respectively. The 


\begin{tabular}{|c|c|c|c|c|c|}
\hline Algorithm & $\hat{\mu}_{t}$ & $\hat{\mu}_{b}$ & $\hat{\mu}_{t}-\hat{\mu}_{b}$ & $\hat{\sigma}_{b}$ & $\hat{\mu}_{S C R}$ \\
\hline CC & 645.20 & 39.46 & 605.74 & 22.56 & 26.85 \\
\hline CE & $1.30 \times 10^{3}$ & 38.97 & $1.26 \times 10^{3}$ & 19.58 & 64.6 \\
\hline NF & $2.97 \times 10^{5}$ & $1.59 \times 10^{4}$ & $2.81 \times 10^{5}$ & $3.28 \times 10^{4}$ & 8.58 \\
\hline NF-A & $1.83 \times 10^{5}$ & $3.53 \times 10^{3}$ & $1.79 \times 10^{5}$ & $1.12 \times 10^{4}$ & 15.18 \\
\hline
\end{tabular}

Table 4.9: Signal to clutter ratio for various algorithms using ARCHER MAST data set

sample mean and variance for the MB methods are much greater than these expected values. While this may partially result from the use of a white noise model rather than the true noise model associated with the data, the large values mostly arise from residual estimation error. These results demonstrate limitations associated with the MB approach. Essentially, the performance associated with these MB methods is limited by the accuracy of the model itself. When significant residual calibration error exists in the data, the MB methods are subject to a decrease in performance because the model does not account for the calibration error. Similarly, the spatial dependence of the calibration terms in the geocorrected and registered data is much more complex than that given in the original uncalibrated and relatively calibrated data models. This problem could be addressed if a geocorrection/registration pixel mapping is provided with the data and the uncalibrated model matched the data as in the cases for the AFRL and HYDICE data. In the HYDICE case, however, it is seen that the detection performance gain using the MB change detection approach relative to $\mathrm{CC}$ and $\mathrm{CE}$ is eliminated when using the generalized model to handle uncalibrated sensor data. This is perhaps a fundamental limitation. 


\subsection{Discussion}

In summary, the MB change detection method is applied to four data sets, each with its own set of challenges and lessons learned. Here, a summary of the important conclusions and results for each data set are summarized.

For the synthetic MODTRAN data set,

1. The data set serves as a proof-of-concept for the model-based approach and has no model mismatch, misregistration/parallax error, or residual calibration error.

2. The automated noise estimation technique produces relatively accurate estimates.

3. The MB method achieves superior detection performance than baseline approaches.

4. The detection performance does not significantly vary for $5 \%, 10 \%$, and true shadow initialization.

5. Clutter leakage resulting from shadow differences is effectively suppressed.

6. Without RT basis bound constraints, detection performance decreases if the subspace dimension $I$ becomes too large. With the bound constraints in place, no additional improvement in detection performance is obtained by increasing $I$ well beyond 10 .

7. The spectral filtering and reflectance subspace methods achieve smoother spectral reflectance estimates. However, these smoother estimates do not necessarily result in increased change detection performance. 
For the AFRL data set,

1. The data set serves as a controlled, field-collected data set for which minimal (if any) misregistration exists and for which the calibration process and noise characteristics are well known.

2. The automated noise estimation technique produces accurate estimates.

3. The MB method achieves superior detection performance than baseline approaches.

4. The detection performance does not significantly vary for $5 \%$ and $10 \%$ shadow initialization.

5. Clutter leakage resulting from shadow differences is effectively suppressed.

6. The user of a signal-dependent noise model improves detection performance over the simpler white noise model.

7. The spectral filtering and reflectance subspace methods achieve smoother spectral reflectance estimates. However, these smoother estimates do not necessarily result in increased change detection performance.

8. The MB method using the uncalibrated and relatively calibrated data models still achieves superior change detection performance over the baseline methods. Initialization of the calibration terms using the NDVI method appears to be a viable approach.

9. Clutter leakage exists in several regions of the image resulting most likely from model mismatch as a result of adjacency effects. 
For the HYDICE data set,

1. The data set serves as an absolutely calibrated, airborne data set for which a small degree of misregistration and/or calibration error may exist.

2. The automated noise estimation technique doe not work well for this data set as a result of the lower spatial resolution of the data.

3. The MB method achieves superior detection performance than baseline approaches.

4. The detection performance does not significantly vary for $5 \%$ and $10 \%$ shadow initialization.

5. Clutter leakage resulting from shadow differences is effectively suppressed.

6. The spectral filtering and reflectance subspace methods achieve smoother spectral reflectance estimates. However, these smoother estimates do not necessarily result in increased change detection performance.

7. Clutter leakage exists in several regions of the image resulting most likely from misregistration or model mismatch effects.

For the ARCHER MAST data set,

1. The data set serves as a relatively calibrated, airborne data set for which a small degree of misregistration may exist and for which significant residual calibration error exists. 
2. The MB method does not achieve superior detection performance than the baseline methods. This result suggests that the MB method is more sensitive to calibration error than the baseline methods.

3. The MB method suffers from poor detection performance as a result of the residual calibration error and violation of the spatial dependence assumption for the calibration terms of the data model. This violation results from the geocorrection applied to the imagery.

4. The adaptive method applied for the calibration terms of the model helps reduce residual calibration error but the detection performance is still poor in comparison with the baseline methods. 


\section{Chapter 5: CONCLUSION AND FUTURE WORK}

\subsection{Conclusion}

A new model-based (MB) approach to hyperspectral change detection has been developed. Previous methods for change detection produce false alarms in the presence of nuisance changes, such as global illumination variation, local illumination variation, and misregistration/parallax error. The physical model employed here explicitly accounts for local illumination variation by incorporating shadow coefficients. Using this model along with statistical noise assumptions, the change detection problem is formulated as a hypothesis test. The resulting generalized likelihood ratio test (GLRT) provides an indicator of change for each pixel. This change detection approach is the first to utilize a physical model, which allows for the use of additional information beyond the statistics of the data for improved detection performance over baseline methods.

An optimization problem is developed using the entire joint data set to determine the maximum likelihood estimates (MLEs) of the model parameters for the GLRT. An alternating optimization algorithm is developed in order to produce a tractable estimation problem for the unknown model parameters. This algorithm operates by 
treating certain elements of the data model as known variables while estimating the remaining parameters.

Additionally, the model-based approach is extended for use on both uncalibrated and relatively calibrated hyperspectral data using assumptions of the collection and calibration procedures of the data. In particular, the relationship between at-sensor spectral radiance and uncalibrated and relatively calibrated data is examined to allow for application of the MB method to a broader set of data. The resulting optimization problem for the extended data models is still tractable assuming a moderately sized data cube is being processed. The AO algorithm applied for the calibrated data model is extended to incorporate estimation of the unknown gain and offset terms associated with both the uncalibrated and relatively calibrated data model.

The MB method is applied to synthetic imagery generated using MODTRAN, tower-based data collected using an in-house AFRL sensor, calibrated airborne HYDICE imagery, and relatively calibrated ARCHER MAST data. Improved detection performance is demonstrated over baseline change detection methods for all data sets except the ARCHER MAST data, which contains significant residual calibration error not accounted for in the modeling approaches developed in this dissertation.

Along with development of the model-based change detection algorithm, a discussion of hyperspectral noise characteristics has been presented here with specific emphasis on the signal-dependence of the noise, which is often ignored. The noise variance and signal-dependence will vary both spatially and spectrally as a result of system characteristics and the calibration process applied to the data. Both supervised and unsupervised methods for estimating noise from the imagery using the 
sample variance in small windows around each pixel in the scene is examined. The estimation technique is tested on AFRL hyperspectral data for which calibration data exists. The estimates obtained using these approaches correspond well with those obtained directly from the calibration data. The model and estimation algorithms presented here provide distinct advantages over other noise estimation methods currently found in the literature and, therefore, offer a promising approach for extracting accurate estimates of signal-dependent noise statistics from hyperspectral imagery.

\section{$5.2 \quad$ Future Work}

The model-based change detection development provided here focuses only on the false alarms resulting from shadow variation in the scene. However, misregistration and parallax error are two other significant sources of false alarm in change detection. Future work will examine expanding the data model and optimization technique to incorporate spatial information. Intuitively, one expects neighboring pixels will likely have similar model parameters, such as reflectance and shadow coefficients. Consequently, one may employ Markov random field concepts or other spatial constraints to help improve parameter estimation and detection performance.

Additional future work will examine application of the uncalibrated and relatively calibrated data models on airborne data where geocorrection is required. The geocorrection is typically applied after the data has been calibrated. As a result, in the geocorrected imagery, the assumption that the gain and offset terms are a function of only one spatial index may be invalid. The warping may be more significant for certain portions of the image than others. In regions where the warping is minimal, a 
change in several rows as a result of the geocorrection may not be significant. For significant warping, an alternative method must be determined. If the mapping applied to the spatial positions is known, one can potentially use this knowledge to determine the spatial dependence of the gain and offset terms. Further work along these lines is required. 


\section{Appendix A: MODTRAN}

\section{A.1 MODTRAN Background}

This research work relies heavily upon the atmospheric modeling tool MODTRAN (MODerate resolution atmospheric TRANsmittance and radiance code) for algorithm initialization and RT subspace generation. MODTRAN is a radiative transport model developed primarily by the Air Force. The program was developed to enable scientists to accurately model the radiative transport process in order to predict the radiance (signal) reaching passive sensors mounted on airborne platforms. Essentially, the user can specify the composition of the atmosphere and resulting illumination conditions using atmospheric models, haze models, cloud models, aerosol models, etc. along with solar position, sensor position, and ground reflectance. The code itself then propagates the sources of radiation (sun, moon, thermal) through the atmosphere and ultimately to the sensor. MODTRAN then outputs useful information about the simulation, such as the total radiance reaching the sensor, the transmission through the atmosphere, and various components that comprise the total radiance reaching the sensor, all of which are given as a function of wavelength $\lambda$. A full technical description of the software can be found in the literature [5, 6]. Here a cursory overview is provided detailing important inputs and outputs for the program as related 
to the physical radiative transfer equations derived from Schott's text and the physical model used for this MB change detection work [76]. While some information is given about the thermal emission terms of MODTRAN, the majority of the development is for the non-thermal region for which the MB change detection algorithm is developed.

\section{A.2 MODTRAN Operation}

In this section, a cursory overview of the inputs and outputs of MODDTRAN is provided as related to a more complex, nonlinear radiative transfer model.

\section{A.2.1 Inputs}

The user provides the simulation parameters for MODTRAN using a tape5 (.tp5) file. The file is a structured ASCII text file, which provides MODTRAN with all the information it needs to perform the simulation. The tape 5 file is broken up into a number of "CARDS" or sections with each card providing information about a specific aspect of the simulation (atmospheric model, sensor/solar viewing geometry, etc.). A full description of all the tape 5 inputs is beyond the scope of this document. For a full description, please see the MODTRAN User's Manual [7]. Several of the more relevant input parameters are discussed here as they more directly relate to the change detection work performed.

- MODEL: The user can choose from one of six geographical-seasonal atmospheric models (such as mid-latitude summer, mid-latitude winter, tropical, etc.) or tell MODTRAN more specific atmospheric model parameters using other CARD inputs. 
- IMULT: This input tells MODTRAN whether or not to execute with multiple scattering. Enabling multiple scattering results in longer simulation time but is typically more accurate. This input significantly affects the magnitude of the path and diffuse radiance terms.

- SURREF: This input defines the reflectance of the target material (and background for standard simulation). If a constant between 0 and 1 is assigned, the reflectance is spectrally constant across all wavelengths with the assigned constant value. The user can also tell MODTRAN to use one of the reflectance spectra contained in the MODTRAN library such as forest, desert, grass, etc.

- IHAZE: This input selects the type of atmospheric extinction and a default meteorological range for the boundary-layer aerosol models only.

- VIS: This input defines the visibility or surface meteorological range in kilometers. This input essentially defines the aerosol extinction of the atmosphere. A default visibility value is assigned for each IHAZE model but the default value can be overridden using this input.

- H20 STR: This input defines a scaling factor for the default water vapor column associated with a given MODEL.

- H1: This input provides the altitude of the sensor in kilometers.

- H2: This input provides the altitude of the target material in kilometers.

- ANGLE: This input provides the zenith angle of the sensor $\left(0^{\circ}\right.$ to $\left.180^{\circ}\right)$ as measured from H1. An input of $180^{\circ}$ corresponds to a nadir viewing angle. The H1, H2, and ANGLE parameters will determine the length of the atmospheric path 
from sensor to target. Note, there are other ways to specify the sensor geometry in MODTRAN. This is simply the method chosen here.

- PARM1: This input provides the relative azimuth angle between the sensor lineof-sight and the sensor-to-sun path, measured from the line of sight, positive east of north between $-180^{\circ}$ to $180^{\circ}$. Note for nadir viewing geometry, this input has no effect on the outputs.

- PARM2: This input specifies the solar zenith angle as measured from the sensor. The angle is measured from normal so an input of $0^{\circ}$ would indicate a solar position directly overhead whereas angles approaching $90^{\circ}$ correspond to solar positions much lower in the sky.

- V1: This input denotes the starting wavelength of the simulation in microns.

- V2: This input denotes the ending wavelength of the simulation in microns.

- DV: This input denotes the spectral sampling of the simulation in microns. The combination of V1, V2, and DV will determine the number of spectral bands in the output.

- FWHM: This input denotes the full-width-half-max in microns of the smoothing function applied before sampling. The user should typically have FWHM $\geq 2 * D V$.

While many more input parameters exist for MODTRAN, those provided here summarize the most important in regards to simulations used for the MB change detection work. 


\section{A.2.2 Outputs}

Upon supplying MODTRAN the tape5 file and running the application, a number of output files are created with various extensions. The most complete output file is the tape6 (.tp6) output. This file contains the majority of simulation information produced by MODTRAN. While this output file is the most complete, it contains more information than needed for the purposes of this work. Consequently, two other output files are considered: the tape7 (.tp7) file and the tape7 output convolved with the scanning function defined by the FWHM parameter (.7sc). The convolved output file provides output in user defined units (i.e. wavelength instead of wavenumbers) at the specified wavelength sampling rate. Consequently, the MODTRAN outputs are read from this file for this work. The first section of the output file contains the simulation information from the tape5 file. After this section, the output information is provided in a series of columns. The output columns are described by:

- WAVLEN: Wavelength given in microns

- TOTAL RAD: Total radiance reaching the sensor. In terms of the other outputs, the total radiance is given by GRND RFLT+SOL SCAT+PTH THRML+SURF EMIS.

- GRND RFLT: The total ground reflected radiance (i.e., the direct radiance + diffuse radiance multiplied by surface reflectance)

- DRCT RFLT: The direct reflected radiance (i.e., the solar direct term only multiplied by surface reflectance)

- SING SCAT: The solar single-scattered radiance term of the path radiance. 
- SOL SCAT: The solar multiple-scattered radiance term of the path radiance. This term includes the SING SCAT and incorporates adjacency effects.

- PTH THRML: The path radiance resulting from thermal emission of the atmospheric path. This term will be zero for visible to short-wave infrared simulations.

- THRML SCT: The thermal scattering term of the path radiance. This term will be zero for visible to short-wave infrared simulations.

- SURF EMIS: The thermal emission of the target. This term will be zero for visible to short-wave infrared simulations.

- TRANS: The transmission of the atmosphere from the target to the sensor.

- REF SOL: The top-of-atmosphere solar irradiance (exo-atmospheric) times the atmospheric transmission down to the target and from the target to the sensor.

- SOL OBS: The total solar irradiance at the altitude of the observer.

Note that all radiance terms are given in units of $W /\left(\mathrm{cm}^{2}-s r-\mu m\right)$. These MODTRAN outputs can be directly related to a complete physical model describing the radiance reaching a remote sensor. For the $\mathrm{MB}$ change detection work, the model is derived for the visible to near infrared portion of the spectrum. As a result, the thermal sources are ignored. Additionally, the change detection model ignores adjacency effects to create a more tractable problem. A more complete description of the radiance reaching the sensor from a target pixel with reflectance $\rho$ that includes 
adjacency is given by [76],

$$
\begin{aligned}
& L\left[m, n, \lambda_{k}\right]=\frac{\pi^{-1} E_{e x}\left[\lambda_{k}\right] \cos (\theta) \tau_{s}\left[\lambda_{k}\right] \tau\left[\lambda_{k}\right] \rho\left[m, n, \lambda_{k}\right]}{1-\rho_{b}\left[m, n, \lambda_{k}\right] S_{a}\left[\lambda_{k}\right]}+\frac{L_{d}\left[\lambda_{k}\right] \tau\left[\lambda_{k}\right] \rho\left[m, n, \lambda_{k}\right]}{1-\rho_{b}\left[m, n, \lambda_{k}\right] S_{a}\left[\lambda_{k}\right]} \\
& \quad+L_{a}\left[m, n, \lambda_{k}\right] \rho_{b}\left[m, n, \lambda_{k}\right]+L_{p}\left[\lambda_{k}\right]
\end{aligned}
$$

where $E_{e x}$ is the exoatmospheric solar irradiance (i.e. solar illumination at the top of Earth's atmosphere), $\tau_{s}$ is the transmission from the top of the atmosphere to the target at $\mathrm{H} 2, \tau$ is the transmission from the target at $\mathrm{H} 2$ to the sensor at $\mathrm{H} 1, \rho_{b}$ is the reflectance of the nearby background, $L_{d}$ is the diffuse solar radiance, $L_{a}$ is path radiance due to adjacent pixels, $L_{p}$ is the path radiance due to solar scattering off the atmosphere, $\theta$ is the relative angle between the surface normal and the incident solar illumination, and $S_{a}$ is the spherical scattering albedo, which describes the multiple scattering effects. The spherical albedo is often thought of as the diffuse reflectivity of the sky to upwelling radiation (i.e. how much radiation the sky reflects back down towards the ground). Writing this full model using terms and definitions from the change detection model results in,

$$
\begin{gathered}
L\left[m, n, \lambda_{k}\right]=\frac{L_{s}\left[\lambda_{k}\right] \cos (\theta) \tau\left[\lambda_{k}\right] \rho\left[m, n, \lambda_{k}\right]}{1-\rho_{b}\left[m, n, \lambda_{k}\right] S_{a}\left[\lambda_{k}\right]}+\frac{L_{d}\left[\lambda_{k}\right] \tau\left[\lambda_{k}\right] \rho\left[m, n, \lambda_{k}\right]}{1-\rho_{b}\left[m, n, \lambda_{k}\right] S_{a}\left[\lambda_{k}\right]} \\
\quad+L_{a}\left[m, n, \lambda_{k}\right] \rho_{b}\left[m, n, \lambda_{k}\right]+L_{p}\left[\lambda_{k}\right]
\end{gathered}
$$

where $L_{s}\left[\lambda_{k}\right]=\pi^{-1} E_{e x}\left[\lambda_{k}\right] \tau_{s}\left[\lambda_{k}\right]$. This represents a more complete model incorporating nonlinear multiple scattering and adjacency effects described by the terms $\left(1-\rho_{b}\left[m, n, \lambda_{k}\right] S_{a}\left[\lambda_{k}\right]\right)$ and $\left(L_{a}\left[m, n, \lambda_{k}\right] \rho_{b}\left[m, n, \lambda_{k}\right]\right)$. Figures A.1 and A.2 provide a visual description of the individual terms in the model.

As the turbidity of the atmosphere grows small $\left(S_{a}\left[\lambda_{k}\right] \ll 1\right)$, the multiple scattering and adjacency effects lessen. In the formulation currently being used for change 


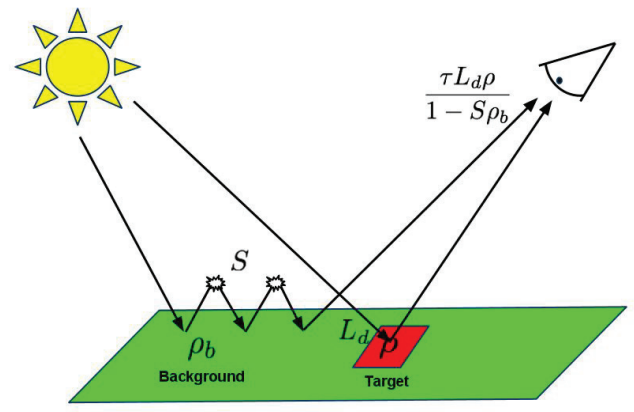

(a) Direct Radiance

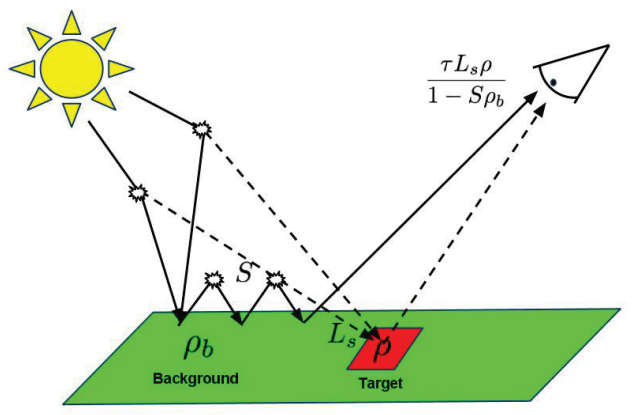

(b) Diffuse Radiance

Figure A.1: Radiative transfer diagrams for direct and diffuse radiance incorporating nonlinear scattering and adjacency

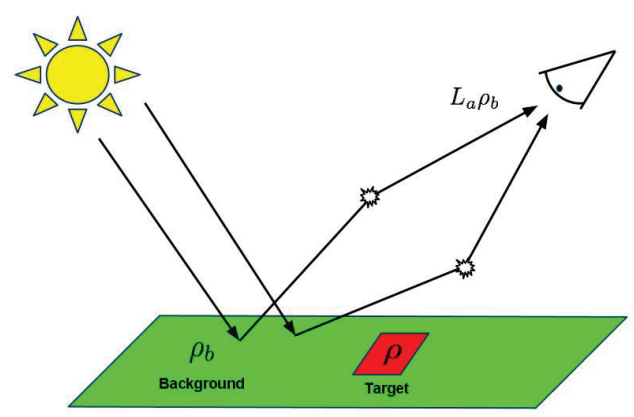

(a) Upwelled radiance due to adjacency

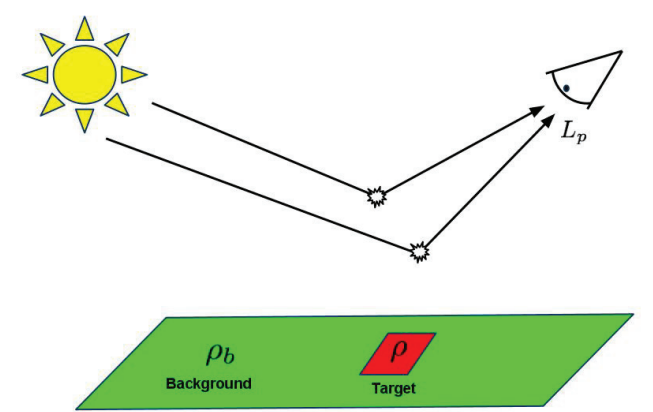

(b) Path Radiance

Figure A.2: Radiative transfer diagrams for upwelled radiance incorporating nonlinear scattering and adjacency 
detection, these effects are ignored resulting in the simpler model given by,

$$
L\left[m, n, \lambda_{k}\right]=L_{s}\left[\lambda_{k}\right] \cos (\theta) \tau\left[\lambda_{k}\right] \rho\left[m, n, \lambda_{k}\right]+L_{d}\left[\lambda_{k}\right] \tau\left[\lambda_{k}\right] \rho\left[m, n, \lambda_{k}\right]+L_{p}\left[\lambda_{k}\right]
$$

However, MODTRAN does not ignore these terms and the user must be aware of the relationship of MODTRAN's outputs with these terms. The output of MODTRAN for total radiance is related to the more complex model given in (A.2) by,

$$
L\left[m, n, \lambda_{k}\right]=\text { GRND RFLT }+ \text { SOL } \mathrm{SCAT}=\text { TOTAL RAD }
$$

where

$$
\text { GRND } \operatorname{RFLT}=\frac{L_{s}\left[\lambda_{k}\right] \cos (\theta) \tau\left[\lambda_{k}\right] \rho\left[m, n, \lambda_{k}\right]}{1-\rho_{b}\left[m, n, \lambda_{k}\right] S_{a}\left[\lambda_{k}\right]}+\frac{L_{d}\left[\lambda_{k}\right] \tau\left[\lambda_{k}\right] \rho\left[m, n, \lambda_{k}\right]}{1-\rho_{b}\left[m, n, \lambda_{k}\right] S_{a}\left[\lambda_{k}\right]}
$$

and

$$
\mathrm{SOL} \operatorname{SCAT}=L_{a}\left[m, n, \lambda_{k}\right] \rho_{b}\left[m, n, \lambda_{k}\right]+L_{p}\left[\lambda_{k}\right]
$$

Additionally, MODTRAN provides

$$
\mathrm{REF} \mathrm{SOL}=E_{e x}\left[\lambda_{k}\right] \tau_{s}\left[\lambda_{k}\right] \tau\left[\lambda_{k}\right]
$$

As mentioned earlier, when running MODTRAN, the user must specify the surface reflectance using the SURREF input. In the most general operating mode, this surface reflectance is used for both the target pixel and all the background, i.e., $\rho_{b}=\rho=$ SURREF. Consequently, in this mode of operation, the MODTRAN world is composed of a single material with the same reflectance for all ground surfaces. In this normal mode of operation, the individual terms for the simpler model given in (A.3) cannot be separated due to the adjacency effects. The terms that can be separated are transmission,

$$
\tau\left[\lambda_{k}\right]=\text { TRANS }
$$


direct solar radiance,

$$
L_{s}\left[\lambda_{k}\right]=\frac{\mathrm{REF} \text { SOL }}{\pi \mathrm{TRANS}}
$$

and the relative angle between a horizontal surface and the solar illumination angle (simply the solar zenith angle)

$$
\theta=\text { PARM2 }
$$

The other terms of the simple model cannot be extracted from the remaining information available. For this reason, an alternative mode of operation available in MODTRAN must be used. In this mode, the user can explicitly define the reflectance (and temperature for thermal simulations) of the target and background separately. By specifying $\rho\left[m, n, \lambda_{k}\right]=1$ and $\rho_{b}\left[m, n, \lambda_{k}\right]=0$, the resulting MODTRAN outputs correspond to,

$$
\begin{aligned}
& \text { GRND } \operatorname{RFLT}=L_{s}\left[\lambda_{k}\right] \cos (\theta) \tau\left[\lambda_{k}\right]+L_{d}\left[\lambda_{k}\right] \tau\left[\lambda_{k}\right] \\
& \text { SOL SCAT }=L_{p}\left[\lambda_{k}\right] \\
& \text { DRCT RFLT }=L_{s}\left[\lambda_{k}\right] \cos (\theta) \tau\left[\lambda_{k}\right]
\end{aligned}
$$

All of the simple model parameters can now be separated using a single MODTRAN simulation as,

$$
\begin{aligned}
L_{p}\left[\lambda_{k}\right] & =\text { SOL SCAT } \\
L_{s}\left[\lambda_{k}\right] & =\frac{\text { REF SOL }}{\pi \text { TRANS }} \\
L_{d}\left[\lambda_{k}\right] & =\frac{\text { GRND RFLT }- \text { DRCT RFLT }}{\text { TRANS }}
\end{aligned}
$$

The direct solar radiance can equivalently be obtained using,

$$
L_{s}\left[\lambda_{k}\right]=\frac{\text { DRCT RFLT }}{\cos (\text { PARM2)TRANS }}
$$


Additionally, if a second MODTRAN simulation is run with $\rho\left[m, n, \lambda_{k}\right]=1$ and $\rho_{b}\left[\Delta m, n, \lambda_{k}\right]=1$, the adjacency parameters can be obtained using,

$$
\begin{aligned}
& \text { SOL SCAT }=L_{a}\left[m, n, \lambda_{k}\right]+L_{p}\left[\lambda_{k}\right] \\
& \text { DRCT RFLT }=\frac{L_{s}\left[\lambda_{k}\right] \cos (\theta) \tau\left[\lambda_{k}\right]}{1-S_{a}\left[\lambda_{k}\right]}
\end{aligned}
$$

Since the direct solar radiance and path radiance terms are known from the previous simulation, the adjacency term $L_{a}$ and the spherical albedo term $S_{a}$ can easily be obtained. While the adjacency terms are not utilized at this time, they may be valuable for future extensions of model-based change detection method. 


\section{Bibliography}

[1] Bruno Aiazzi et al. Noise modeling and estimation of hyperspectral data from airborne imaging spectrometers. Annals of Geophysics, 49(1):1-9, February 2006.

[2] W. S. Aldrich, M. E. Kappus, and R. G. Resmini. HYDICE post-flight processing. In Proc. SPIE, volume 2758, pages 354-363, 1996.

[3] R. W. Basedow, W. S. Aldrich, J. E. Colwell, and W. D. Kinder. HYDICE system performance - An update. In Proc. SPIE, volume 2821, pages 76-84, 1996.

[4] R. W. Basedow, D. C. Armer, and M. Anderson. HYDICE system: Implementation and performance. volume 2480 of Proc. SPIE, pages pp. 258 - 267, 1995.

[5] A. Berk, L. Bernstein, and D. Robertson. MODTRAN: A moderate resolution model for lowtran 7. Tech. report GL-TR-89-0122, Geophysics Lab, Bedford, MA, 1989.

[6] A. Berk et al. MODTRAN4: Radiative transfer modeling for atmospheric correction. volume 3756 of Proc. SPIE, pages 348 - 353, 1999.

[7] A. Berk et al. MODTRAN4 Version 3 Revision 1 USER'S MANUAL. Software manual, Air Force Research Laboratory, Space Vehicles Directorate, Hanscom Air Force Base, 2003.

[8] L. S. Bernstein et al. A new method for atmospheric correction and aerosol optical property retrieval for VIS-SWIR multi- and hyperspectral imaging sensors: QUAC (QUick Atmospheric Correction). IEEE Geoscience and Remote Sensing Symposium, 5:4549 - 4552, 2005.

[9] Dimitri P. Bertsekas. Nonlinear Programming. Athena Scientific, Belmont, MA, second edition, 1999.

[10] James C. Bezdek and Richard J. Hathaway. Some notes on alternating optimization. AFSS '02: Proceedings of the 2002 AFSS International Conference on Fuzzy Systems. Calcutta, pages 288-300, London, UK, 2002. Springer-Verlag. 
[11] José M. Bioucas-Dias and José M. P. Nascimento. Estimation of signal subspace on hyperspectral data. volume 5982, page 59820L. SPIE, 2005.

[12] J. Blackburn et al. Feature aided tracking with hyperspectral imagery. volume 6699 of Proc. SPIE, pages 66990S1 - 66990S12, 2007.

[13] Lorenzo Bruzzone and Diego Fernàndez Prieto. Automatic analysis of the difference image for unsupervised change detection. IEEE Trans. Geoscience and Rem. Sens., 38(3):1171-1182, 2000.

[14] Arthur E. Burgess. The Rose model, revisited. Journal of Opt. Soc. of Amer. A, 16(3):633 -646, March 1999.

[15] M. J. Carlotto. Non-linear mean-square estimation with applications in remote sensing. volume 2758 of Proc. SPIE, pages $206-217,1996$.

[16] Kartik Chandra and Glenn Healey. Recovering surface properties for hyperspectral scenes. volume 6233, page 62331H. SPIE, 2006.

[17] Kartik Chandra and Glenn Healey. Using coupled subspace methods for reflectance/illumination separation. IEEE Trans. on Geoscience and Remote Sensing, 46(1):284 - 290, 2008.

[18] Keming Chen et al. Change detection based on adaptive markov random fields. 19th Intern. Conf. on Patt. Recog., pages 1-4, Dec. 2008.

[19] C. Clifton. Change detection in overhead imagery using neural networks. App. Intell., 18:215-234, 2003.

[20] T. F. Coleman and Y. Li. A reflective newton method for minimizing a quadratic function subject to bounds on some of the variables. SIAM Journal on Optimization, 6(4):1040 - 1058, 1996.

[21] J. B. Collins and C. E. Woodcock. An assessment of several linear change detection techniques for mapping forest mortality using multi-temporal landsat tm data. Remote Sens. Environ., 56:66-77, 1996.

[22] R. Collins, A. Lipton, and T. Kanade. Introduction to the special section on video surveillance. IEEE Trans. Patter Analy. Mach. Intell., 22(8):745 - 746, 2000 .

[23] P. Coppin and M. Bauer. Digital change detection in forest ecosystems. 13:207 - 234, 1996.

[24] Richard Courant and David Hilbert. Methods of Mathematical Physics, volume 1. Interscience Publisher, Inc., New York, 1953. 
[25] DALSA Digital Imaging. Pantera TF 1M60 area scan cameras. http://www. dalsa.com/, Aug 2005.

[26] Oliver E. Dial. Ccd performance model. volume 1479, pages 2-11. SPIE, 1991.

[27] Michael T. Eismann and Joseph Meola. Use of spectral clustering to enhance clutter suppression for hyperspectral change detection. volume 6565 of Proc. SPIE, pages 65651T-1 - 65651T-12, 2007.

[28] Michael T. Eismann, Joseph Meola, A. Stocker, S. Beaven, and A. Schaum. Hyperspectral change detection in the presence of diurnal and seasonal variations. Transactions on Geoscience and Remote Sensing, 46(1):237 - 249, 2008.

[29] Michael T. Eismann, Joseph Meola, Alan D. Stocker, Scott G. Beaven, and Alan P. Schaum. Airborne hyperspectral detection of small changes. Appl. Opt., 47(28):F27-F45, Oct 2008.

[30] M.T. Eismann and R.C. Hardie. Application of the stochastic mixing model to hyperspectral resolution enhancement. Geoscience and Remote Sensing, IEEE Transactions on, 42(9):1924 - 1933, 2004.

[31] William Fitzsimmons. Everything has changed, 2009. Naim Label.

[32] Roger Fletcher. Practical Methods of Optimization. John Wiley and Sons, New York, NY, second edition, 1987.

[33] R. N. Fraser. Hyperspectral remote sensing of turbidity and chlorophyll a among Nebraska Sand Hills Lakes. International Journal of Remote Sensing, 19(8):15791589, 1998.

[34] Lian-Ru Gao, Bing Zhang, Xia Zhang, Wen-Juan Zhang, and Qing-Xi Tong. A new operational method for estimating noise in hyperspectral images. Geoscience and Remote Sensing Letters, IEEE, 5(1):83 -87, jan. 2008.

[35] P. E. Gill, W. Murray, and M. H. Wright. Practical Optimization. Academic Press, London, 1981.

[36] A.A. Green, M. Berman, P. Switzer, and M.D. Craig. A transformation for ordering multispectral data in terms of image quality with implications for noise removal. IEEE Trans. on Geoscience and Remote Sensing, 26(1):65 -74, jan. 1988.

[37] Geoffrey G. Hazel. Multivariate gaussian MRF for multispectral scene segmentation and anomaly detection. IEEE Trans. Geoscience and Rem. Sens., 38(3):1199-1211, 2000. 
[38] Glenn Healey and R. Kondepudy. Radiometric CCD camera calibration and noise estimation. IEEE Trans. Pattern Anal. Machine Intell., 16:267-276, 1994.

[39] Glenn Healey and Raghava V. Kondepudy. Modeling and calibrating ccd cameras for illumination-insensitive machine vision. volume 1614, pages 121-132. SPIE, 1992.

[40] Gerald Holst. CCD Arrays Cameras and Displays. JCD Publishing, Winter Park, FL, second edition, 1998.

[41] A. Huertas and R. Nevatia. Detecting changes in aerial views of manmade structures. Image Vis. Comp., 18(8):583 - 596, 2000.

[42] B. R. Hunt and T. M. Cannon. Nonstationary assumptions for gaussian models of images. IEEE Trans. Syst., Man, Cybern., SMC-6:876-882, Dec. 1976.

[43] E. Karpouzli and T. Malthus. The empirical line method for the atmospheric correction of IKONOS imagery. International Journal of Remote Sensing, 24(5):1143-1150, 2003.

[44] T. Kasetkasem and P. K. Varshney. An image change detection algorithm based on markov random field models. IEEE Trans. Geoscience and Rem. Sens., 40(8):1815-1823, 2002.

[45] Steven M. Kay. Fundamentals of Statistical Signal Processing: Detection Theory. Prentice Hall, Upper Saddle River, NJ, 1998.

[46] John P. Kerekes and Jerrold E. Baum. Hyperspectral imaging and system modeling. Lincoln Laboratory Journal, 14(1):117-130, 2003.

[47] D. Landgrebe. Hyperspectral image data analysis. Signal Processing Magazine, IEEE, 19(1):17 -28, jan. 2002.

[48] Terrence S. Lomheim and Erich D. Hernandez-Baquero. Translation of spectral radiance levels, band choices, and signal-to-noise requirements to focal plane specifications and design constraints. volume 4486, pages 263-307. SPIE, 2002.

[49] D. Manolakis and G. Shaw. Detection algorithms for hyperspectral imaging applications. Signal Processing Magazine, IEEE, 19(1):29 -43, jan. 2002.

[50] Dimitris Manolakis, David Marden, and Gary A. Shaw. Hyperspectral image processing for automatic target detection applications. Lincoln Laboratory Journal, 14(1):79-116, 2003.

[51] A. Margalit, I.S. Reed, and R.M. Gagliadri. Adaptive optical target detection using correlated images. IEEE Trans. Aerospace Electron. Sys., AE-38:394-405, May 1985. 
[52] J. Martin-Herrero. Comments on "A New Operational Method for Estimating Noise in Hyperspectral Images". Geoscience and Remote Sensing Letters, IEEE, 5(4):705-709, oct. 2008.

[53] J. Meola, M. T. Eismann, R. L. Moses, and J. N. Ash. Detecting changes in hyperspectral imagery using a model-based approach. Geoscience and Remote Sensing, IEEE Transactions on, 49(7):2647 -2661, July 2011.

[54] Joseph Meola. Analysis of hyperspectral change detection as affected by vegetation and illumination variation. Master's thesis, University of Dayton, Dayton, $\mathrm{OH}$, 2006.

[55] Joseph Meola and Michael T. Eismann. Image misregistration effects on hyperspectral change detection. volume 6966 of Proc. SPIE, pages $69660 \mathrm{Y}-1-$ 69660Y-10, 2008.

[56] Joseph Meola et al. Analysis of hyperspectral change detection as affected by vegetation and illumination variation. volume 6565 of Proc. SPIE, pages 65651S1 - 65651S-12, 2007.

[57] Shirley Morillo-Contreras, Miguel Velez-Reyes, and Shawn D. Hunt. A comparison of noise reduction methods for image enhancement in classification of hyperspectral imagery. volume 5806, pages 361-370. SPIE, 2005.

[58] G. Nagy et al. Volume and surface area distributions of cracks in concrete. Proc. Visual Form, pages $759-768,2001$.

[59] S. Negahdaripour. Revised definition of optical flow: Integration of radiometric and geometric cues for dynamic scene analysis. IEEE Trans. Patt. Anal. Mach. Intell., 20(9):961-978, Sept. 1998.

[60] A.A. Nielsen. The regularized iteratively reweighted mad method for change detection in multi- and hyperspectral data. Image Processing, IEEE Transactions on, 16(2):463-478, 2007.

[61] Allan A. Nielsen, Knut Conradsen, and James J. Simpson. Multivariate alteration detection (MAD) and MAF postprocessing in multispectral bitemporal image data: New approaches to change detection studies. Remote Sens. Environ., 64:1-19, 1998.

[62] Melissa L. Nischan, John P. Kerekes, Jerrold E. Baum, and Robert W. Basedow. Analysis of hydice noise characteristics and their impact on subpixel object detection. volume 3753, pages 112-123. SPIE, 1999. 
[63] Timothy Perkins et al. Retrieval of Atmospheric Properties from Hyper- and Multi-spectral Imagery with FLAASH Atmospheric Correction Algorithm. In Proc. SPIE, volume 5979, pages 59790E-1 - 59790E-11, 2005.

[64] B. Phong. Illumination for computer generated pictures. Commun. ACM, 18:311-317, 1975.

[65] Richard J. Radke et al. Image change detection algorithms: A systematic survey. IEEE Trans. on Image Processing, 14(3):294-307, 2005.

[66] I. S. Reed and X. Yu. Adaptive multiple-band CFAR detection of an optical patter with unknown spectral distribution. IEEE Trans. Acoust. Speech Signal Process., 38:1760 - 1770, 1990.

[67] D. Rey et al. Automatic detection and segmentation of evolving processes in 3d medical images: Application to multiple sclerosis. Med. Image Anal., 6(2):163179, 2002.

[68] Lee J. Rickard et al. HYDICE: an airborne system for hyperspectral imaging. In Proc. SPIE, volume 1937, pages 173-179, 1993.

[69] R. E. Roger and J. F. Arnold. Reliably estimating the noise in AVIRIS hyperspectral images. International Journal of Remote Sensing, 17(10):1951-1962, 1996.

[70] Louis L. Scharf and Donald W. Tufts. Rank reduction for modeling stationary signals. IEEE Transactions on Acoustics, Speech, and Signal Processing, 35(3):350-355, March 1987.

[71] A. Schaum et al. Hyperspectral change detection in high clutter using elliptically contoured distributions. volume 6565 of Proc. SPIE, pages 656515-1 - 656515$12,2007$.

[72] A. Schaum and M. McHugh. Analytic methods of image registration: Displacement estimation and resampling. Technical Report 9298, Naval Research Lab, 1991.

[73] A. Schaum and A. Stocker. Long-interval chronochrome target detection. Proc. 1997 Internation Symp. on Spec. Sensing Res., pages 1 - 14, 1997.

[74] A. Schaum and A. Stocker. Hyperspectral change detection and supervised matched filtering based on covariance equalization. volume 5425 of Proc. SPIE, pages 77-90, 2004.

[75] A. Schaum and A. Stocker. Linear chromodynamics models for hyperspectral target detection. IEEE Aerospace Conference, pages 77-90, February 2003. 
[76] John R. Schott. Remote Sensing: The Image Chain Approach. Oxford University Press, New York, NY, second edition, 2007.

[77] Susan M. Schweizer and José M. F. Moura. Hyperspectral imagery: Clutter adaptation in anomaly detection. IEEE Trans. on Inf. Theory, 46(5):1855-1871, 2000 .

[78] G. Shaw and D. Manolakis. Signal processing for hyperspectral image exploitation. Signal Processing Magazine, IEEE, 19(1):12 -16, jan. 2002.

[79] Ashbindu Singh. Digital change detection techniques using remotely-sensed data. International Journal of Remote Sensing, 10(6):989-1003, 1989.

[80] D.W.J. Stein, S.G. Beaven, L.E. Hoff, E.M. Winter, A.P. Schaum, and A.D. Stocker. Anomaly detection from hyperspectral imagery. Signal Processing Magazine, IEEE, 19(1):58 -69, January 2002.

[81] Brian Stevenson, Rory O'Connor, William Kendall, Alan Stocker, William Schaff, Drew Alexa, John Salvador, Michael Eismann, Kenneth Barnard, and John Kershenstein. Design and performance of the civil air patrol archer hyperspectral processing system. volume 5806, pages 731-742. SPIE, 2005.

[82] R.L. Streit, M.L. Graham, and M.J. Walsh. Tracking in hyper-spectral data. volume 2 of Proceedings of the Fifth International Conference on Information Fusion, pages 852-859, 2002.

[83] P. Suen, Glenn Healey, and David Slater. The impact of viewing geometry on material discriminability in hyperspectral images. IEEE Trans. on Geoscience and Remote Sensing, 39(7):1352-1360, 2001.

[84] J. Theiler and S. Perkins. Resampling approach for anomalous change detection. volume 6565 of Proc. SPIE, pages 65651U-1 - 65651U-12, 2007.

[85] J. Theiler and S. Perkins. Proposed framework for anomalous change detection. Proc. of the ICML Workshop on Mach. Learning Alg. for Surv. and Event Det., pages 7-14, June 2006.

[86] James Theiler. Quantitative comparison of quadratic covariance-based anomalous change detectors. Applied Optics, 47(28):F12-F26, 2008.

[87] S. Watanabe, K. Miyajima, and N. Mukawa. Detecting changes of buildings from aerial images using shadow and shading model. Proc. ICPR, pages 1408 - 1412, 1998.

[88] William M. Weibel, Ara Oshagan, and Alan D. Stocker. ISIS Version 5: User's Manual. Software manual, Space Computer Corp., Los Angeles, CA, 2006. 